Composition and function of the Crumbs protein complex in the mammalian retina 
Composition and function of the Crumbs protein complex in the mammalian retina

The studies in this thesis were performed at the department of Human Genetics, Nijmegen Centre for Life Sciences, Radboud University Nijmegen Medical Centre, Nijmegen, The Netherlands

ISBN/EAN: 978-90-9022660-6

(C) 2008 I. Gosens

All rights reserved. No part of this thesis may be reproduced or transmitted in any form or by any means without prior permission in writing from the copyright owner.

Lay-out: Ilse Gosens

Cover: Alwin Derijck

Photograph: Sand-ball making crabs, Cape Tribulation, Queensland, Australia.

Printed by:

Printpartners Ipskamp B.V., Enschede 


\title{
Composition and function
}

\section{of the Crumbs protein complex}

\section{in the mammalian retina}

\section{Een wetenschappelijke proeve op het gebied van de Medische Wetenschappen}

\author{
Proefschrift
}

ter verkrijging van de graad van doctor aan de Radboud Universiteit Nijmegen, op gezag van de rector magnificus prof. mr. S.C.J.J. Kortmann volgens besluit van het College van Decanen in het openbaar te verdedigen op dinsdag 11 maart 2008 om $\mathbf{1 3 . 3 0}$ uur precies

door

Ilse Gosens

geboren op 6 mei 1979

te Schiedam 


\section{Promotor}

Prof. dr. F.P.M. Cremers

\section{Copromotor}

Dr. R. Roepman

\section{Manuscriptcommissie}

Prof. dr. R.E. Brock (voorzitter)

Dr. W.J.A.J. Hendriks

Prof. dr. E. Knust (Max-Planck-Institute, Dresden, Germany) 


\section{CONTENTS}

List of protein names and their aliases $\quad 7$

$\begin{array}{ll}\text { Abbreviations } & 8\end{array}$

Chapter 1. General introduction $\quad 11$

1. Visual perception 13

2. Development and structure of the retina 14

2.1 Sensory neurons and the phototransduction cascade $\quad 15$

2.2 Interneurons and projecting neurons $\quad 17$

2.3 Müller glia cells $\quad 17$

3. Inherited retinal dystrophies 18

4. Approach to determine the composition and function 20

of the Crumbs protein complex
5. Aim and outline of this thesis

Chapter $2 \quad 27$

MPP5 recruits MPP4 to the CRB1 complex in the retina

\section{Chapter 3}

MPP1 links the Usher protein network and the Crumbs protein complex in the retina

\section{Chapter 4}

Syntenin-1 is a novel binding partner of cell-polarity

determinant MPP5 in the mammalian retina

\section{Chapter 5}

FERM protein EPB41L5 is a novel member of the mammalian CRB-MPP5 polarity complex

Chapter 6. The composition and function of the Crumbs protein complex in the mammalian retina

1. Introduction

Crumbs and retinal degeneration

2. The intracellular Crumbs protein complex 
3. The function of the Crumbs complex at the SAR in the retina

3.1 The Crumbs complex in synapses

3.2 The Crumbs protein complex functions in cilia and basal bodies

3.3 The Crumbs complex and vesicular transport

4. Animal models for Crumbs and Crumbs complex members

5. Conclusion

1. Crumbs recruits a subcortical protein scaffold

2. The role of the extracellular domain of Crumbs

3. Differential localization of Crumbs proteins in the retina

4. Disease causing mutations and the Crumbs complex

5. Crumbs complex components as modifier genes

Summary

Samenvatting

List of publications

Curriculum Vitae

Dankwoord

Color figures 
List of protein names and aliases

\begin{tabular}{|c|c|c|}
\hline & $\underline{\mathrm{HUGO}}$ & $\underline{\text { Alias }}$ \\
\hline 1. & MPP1 & $\mathrm{p} 55$ \\
\hline 2. & MPP2 & DLG-2 \\
\hline 3. & MPP3 & DLG-3 \\
\hline 4. & MPP4 & DLG-6 \\
\hline 5. & MPP5 & Pals1 \\
\hline 6. & MPP6 & Pals2 or VAM-1 \\
\hline 7. & MPP7 & FLJ32798 \\
\hline 8. & LIN7A & Veli-1 or Mals-1 \\
\hline 9. & LIN7B & Veli- 2 or Mals- 2 \\
\hline 10. & LIN7C & Veli- 3 or Mals- 3 \\
\hline 11. & C16orf70 & LIN10 \\
\hline 12. & CASK & LIN2 \\
\hline 13. & DLG1 & SAP97 \\
\hline 14. & DLG2 & PSD-93 \\
\hline 15. & DLG3 & SAP102 \\
\hline 16. & DLG4 & PSD-95 or SAP90 \\
\hline 17. & DLG5 & P-dlg \\
\hline 18. & DLG7 & KIAA0008 or HURP \\
\hline 19. & EPB41 & $4.1 \mathrm{R}$ \\
\hline 20. & EPB41L1 & KIAA0338 \\
\hline 21. & EPB41L2 & $4.1 \mathrm{G}$ \\
\hline 22. & EPB41L3 & 4.1B or DAL1 \\
\hline 23. & EPB41L4A & NBL4 \\
\hline 24. & EPB41L4B & EHM2 \\
\hline 25. & EPB41L5 & KIAA1548 \\
\hline 26. & SDCBP & Syntenin-1 or MDA-9 \\
\hline 27. & SDCBP2 & Syntenin-2 \\
\hline 28. & INADL & PATJ \\
\hline 29. & MPDZ & MUPP1 \\
\hline 30. & PARD6A & PAR6A or PAR6C \\
\hline 31. & PARD6B & PAR6B \\
\hline 32. & PARD6G & PAR6G \\
\hline 33. & TJP1 & $\mathrm{ZO}-1$ \\
\hline 34. & TJP2 & $\mathrm{ZO}-2$ \\
\hline 35. & TJP3 & $\mathrm{ZO}-3$ \\
\hline 36. & DFNB31 & Whirlin or CIP98 \\
\hline 37. & VLN2 & Villin2 or Ezrin \\
\hline
\end{tabular}




\section{Abbreviations}

aa

amino acid

aPKC

atypical Protein Kinase C

$\mathrm{BB}$

basal body

$\mathrm{CC}$

connecting cilium

CRB1

Crumbs homologue 1

EPB41

erythrocyte membrane protein band 4.1

FERM

4.1, ezrin, radixin, moesin

GCL

ganglion cell layer

GST

glutathione-S-transferase

IP

immunoprecipitation

IS

inner segments

IPL

inner plexiform layer

LCA

Leber congenital amaurosis

MAGUK membrane-associated guanylate kinase

MDCK Madin Darby canin kidney

MPP

membrane palmitoylated protein

MUPP1

Multi-PDZ domain protein -1

OLM

outer limiting membrane

ONL

outer nuclear layer

OPL

outer plexiform layer

OS

outer segments

PALS1 Protein Associated with Lin-7 -1

PAR

Partition defective

PATJ

Protein Associated with Tight Junctions

PBM

PDZ binding motif

PCP

Planar Cell Polarity

PCR

polymerase chain reaction

PDZ

PSD-95, DLG1, ZO-1

RP

retinitis pigmentosa

RPE

retinal pigment epithelium

$\mathrm{SH} 3$

Src homology 3

SNP

single nucleotide polymorphism

YMO-1

Yurt/ Mosaic eyes-like 1

ZO-1

Zonula Occludens -1 



\section{CHAPTER 1}

General introduction 



\section{Visual perception}

Our eyes are important sensory organs that allow us to appreciate the beauty around us, to read, gain knowledge and to communicate with each other by reading expressions on faces and making eye-contact. Vision is crucial for our daily routine and therefore, when taken away by a blinding disease, the quality of life is severely impaired.

Although all parts of the eye are important for perceiving a good image, the part that initiates the visual process is the retina. The retina is a neuronal tissue of about 0.4 millimeter thick, decorating the back of the eye, which is directly stimulated by reflected light from objects in the world around us. Light enters the eye passing the cornea and the pupil, the small opening of the iris, and is refracted by the lens to continue through the vitreous humor and neuronal layers of the retina to finally reach the photoreceptors that are present at the back of the retina (Fig. 1).

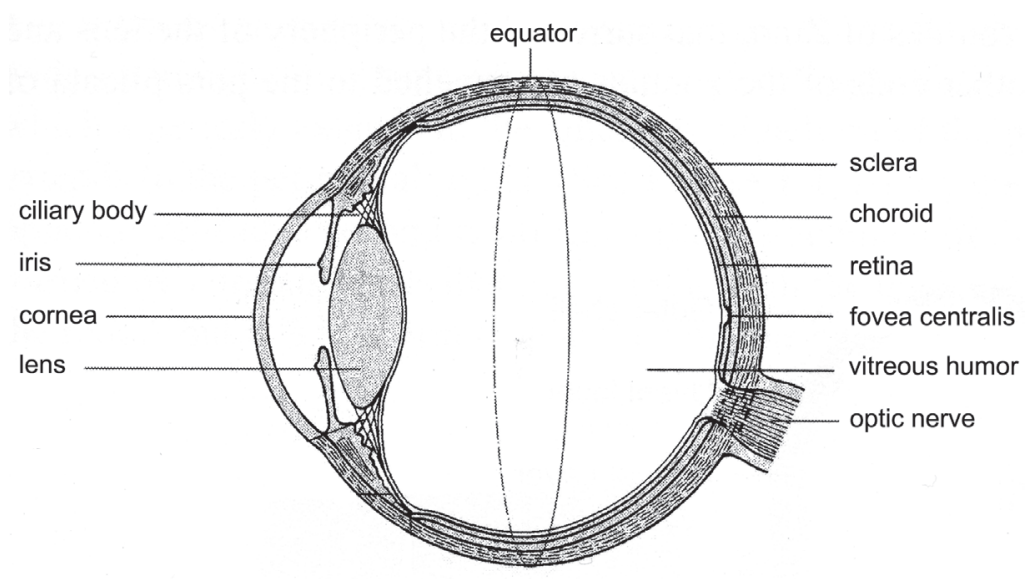

Fig. 1 Anatomy of the eye (adapted from http://www.myopia.manual.de).

Photoreceptors are neurons that are sensitive to light and capable of amplifying and transmitting visual signals. Biochemical processes in the photoreceptors convert the light into electrical impulses that are transferred by several types of neurons to the optic nerve and into the visual brain cortex for further processing ${ }^{1}$. At a central region of the retina, the fovea, the cell bodies of the proximal retinal neurons are shifted to the side, enabling light to travel to the photoreceptors relatively undistorted. At other parts of the retina, light must travel through all neuronal layers before reaching the photoreceptors ${ }^{1}$. 


\section{Development and structure of the retina}

During development of a vertebrate embryo, the retina is formed from the neural tube and is therefore actually a part of the brain. Optic vesicles arise from two sides of the developing neural tube and subsequently fold back upon themselves to form the optic cup (Fig. 2).

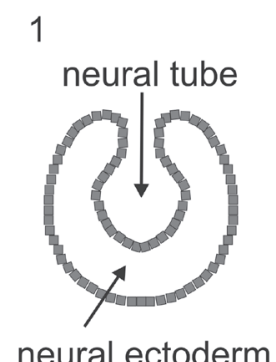

neural ectoderm
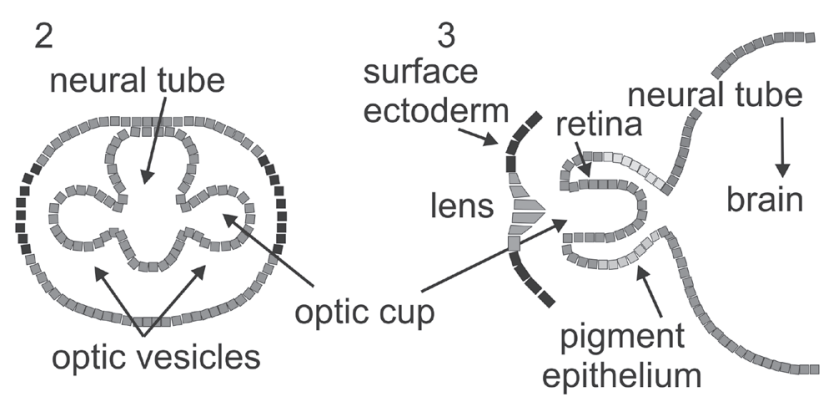

Fig. 2 Neural development of the retina (adapted from http://webvision.med. utah.edu/anatomy.html).

The interior of the optic cup will become the retina with all its different cell types while the outside remains a monolayer of epithelial cells (the retinal pigment epithelium, RPE). The inner epithelial layer, the neuroepithelium, gives rise to all cell types present in the retina. Through cell divisions and subsequent migration, all cells are correctly positioned. Each cell type appears at a different time point during development, with ganglion cells to be the first to exit the cell cycle and start to differentiate. Then amacrine neurons, horizontal cells and cone photoreceptors start to develop, followed by rod photoreceptors and bipolar cells. In the final stage, the Müller glial cells arise ${ }^{2,3}$. All cell types are arranged in a highly organized manner and their cell compartments appear as ten distinct layers in histological sections (Fig. 3).

The RPE cells that are directly attached to the choroid, line the posterior part of the eye in a single layer and form a barrier between the retina and the choroidal circulation. The RPE is involved in exchange of nutrients and waste products. Furthermore, these cuboidal cells absorb any light not captured by the retina that could disrupt a sharp visual image ${ }^{1}$. RPE cells are filled with melanin, which serves a photoprotective role by absorbing radiation and scavenging free radicals and reactive 
oxygen species (ROS) ${ }^{4-7}$. In addition, the RPE phagocytoses discs that are discarded at the tips of photoreceptors and performs a crucial step in the phototransduction cascade, the regeneration of photopigment. In humans, 100 from a total of 1000 rod photoreceptor discs are shed daily.

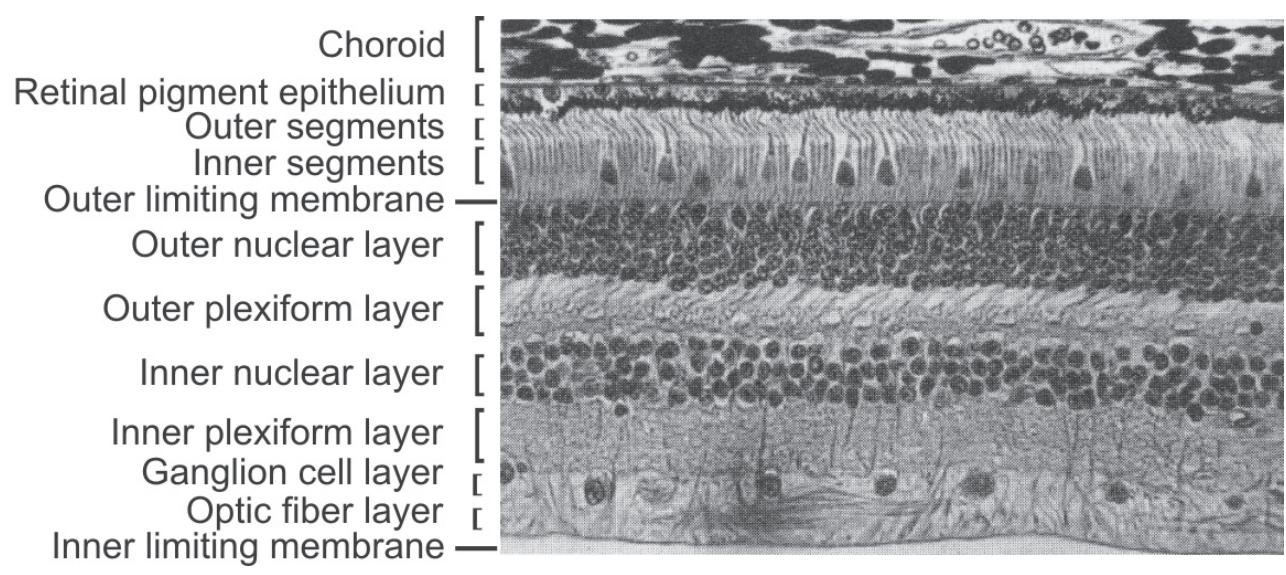

Fig. 3 Layered structure of the neural retina (monkey) and the adjoining RPE and choroid (adapted from thesis A.I. den Hollander).

The remaining nine layers form the neural retina: 1. the photoreceptor layer, comprising the outer and inner segments of rods and cones; 2 . the outer limiting membrane (OLM), not representing a genuine membrane but a region where photoreceptors contact each other as well as Müller glial cells; 3 . the outer nuclear layer (ONL), formed by the nuclei of rods and cones; 4. the outer plexiform layer (OPL), comprised of the synapses of photoreceptors that connect to horizontal, amacrine and bipolar cells; 5. the inner nuclear layer, containing the cell bodies of interneurons and Müller glial cells; 6 . the inner plexiform layers, composed of the synapses between interneurons and ganglion cells; 7 . the ganglion cell layer, containing the cell bodies of these neurons; 8 . the optic fiber layer, composed of the axons of ganglion cells and 9. the inner limiting membrane, formed by the projections of the Müller glial cells (Fig. 4).

\subsection{Sensory neurons and the phototransduction cascade}

Two types of photoreceptors, rods and cones, are involved in acquiring vision. Cones, conical-shaped cells, are concentrated in the fovea and provide spatial resolution. There are three types of cones, each responsive to photons of a different range of wavelengths. They form the basis 


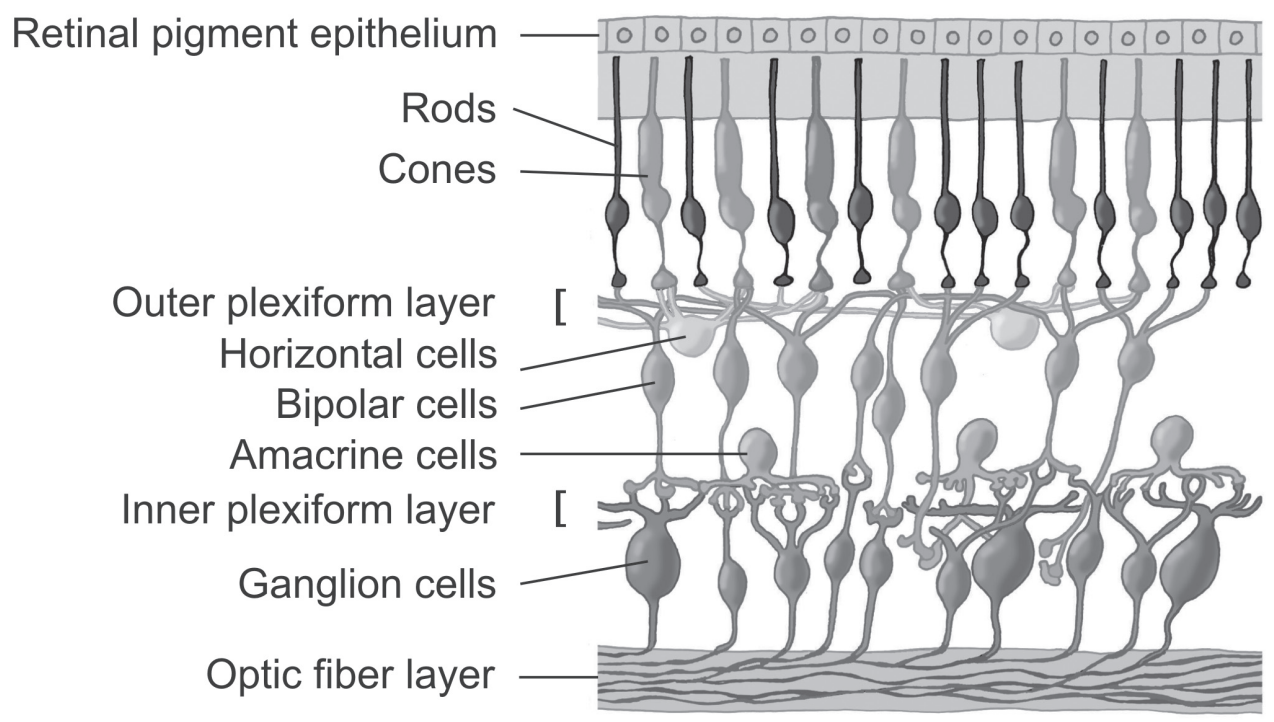

Fig. 4 Cellular structure of the retina (adapted from http://webvision.med. utah.edu/).

for color vision and are used for daytime vision. Rods are cylindrically shaped cells that are involved in night vision and are more sensitive to light than cones. Rods contain more visual pigment than cones, enabling them to capture more light and amplify light signals stronger. A single photon can evoke a detectible electrical signal in rods, whereas hundreds of photons must be absorbed by a cone to evoke a similar response. The number of connections rods and cones make with other neurons in the retina also account for their differences in performance. Many rods e.g. converge onto a single bipolar cell, strengthening the response evoked by light and increasing the ability to detect dim light, whereas fewer cones converge on each bipolar cell, providing better spatial resolution ${ }^{1}$. A human retina contains about 6.4 million cones and $110-125$ million rods ${ }^{8}$. The visual pigment that absorbs photons is embedded within the membranes of discs, the lamellar structures in the outer segment of the photoreceptor. New discs are continuously formed at the base of the outer segment, where a cilium (connecting cilium) connects the outer segment to the inner segments and the rest of the cell body, while discs at the distal end are being shed and phagocytosed by the RPE. Light activates a cascade of biochemical reactions in the photoreceptors called the phototransduction cascade. It activates rhodopsin by inducing isomerization of the covalently attached chromophore 11-cis-retinal to all-trans-retinal. 
The activated opsin results in activation of $\mathrm{G}$-protein transducin, which in its turn activates cyclic GMP phosphodiesterase (PDE). The resulting decrease in cGMP levels closes the cGMP gated cation channels in the outer segment plasma membrane, causing hyperpolarization of photoreceptors that attenuates neurotransmitter release and propagates further signal transduction to the remaining neurons in the retina. In addition, a decline in intracellular calcium activates a negative feedback loop that allows the photoreceptors to recover. In order to restore light sensitivity of the photopigments, all-trans-retinal must be converted back to 11-cisretinal through a multistep pathway, called the visual cycle (reviewed in reference 9).

\subsection{Interneurons and projecting neurons}

Visual information is transferred from photoreceptors to the ganglions cells via three classes of interneurons: bipolar, horizontal and amacrine cells. These interneurons do not simply transmit signals, but combine the signals from several photoreceptors in such a way that the electrical responses evoked in ganglion cells translate to the precise spatial and temporal patterns of light from the visual input. A bipolar cell has dendritic processes in the outer synaptic layer and receives signals from either rods or cones, but never from both. Bipolar cell nuclei are found in the inner nuclear layer and the axon terminals in the inner synaptic layer, where they contact the processes of both amacrine and ganglion cells. Horizontal cells provide lateral inhibition, which acts to enhance spatial differences in photoreceptor activation at the level of bipolar cells. The axons of ganglion cells come together at the optic disc to form the optic nerve, which projects to the brain. Recently, a subset of retinal ganglion cells have been observed to contain melanopsin and function in entrainment of the circadian rhythm and pupil light reflexes ${ }^{10,11}$.

\subsection{Müller glial cells}

Müller glial cells are specialized radial glial cells, which span the entire retina and contact all retinal neuronal cell bodies and processes as well as blood vessels. They are responsible for maintaining homeostasis of the retinal extracellular environment and regulate the flow of ions, water, neurotransmitter molecules, and maintain the $\mathrm{pH}^{12}$. They provide retinal neurons with nutrients such as lactate and pyruvate and remove metabolic waste products ${ }^{13}$. In addition, Müller glial cells participate in 
the recycling of photopigments ${ }^{9,14}$. All-trans-retinal that is released during regeneration of activated opsin is converted to all-trans-retinol in photoreceptors. All-trans-retinol is released by rods and cones into the extracellular space and taken up by Müller glial cells. Different enzymes in Müller glial cells yield 11-cis-retinal through several conversion steps, which is subsequently released and taken up by cones to generate cone opsin pigment. This pathway is suggested to be responsible for sustained daylight vision in vertebrates ${ }^{14}$.

Müller glial cells also influence the life and death of retinal neurons and photoreceptors by releasing neurotrophic factors or mediating the effects of these factors. Neurotrophic factors may protect photoreceptors from cell death directly (via bFGF) or indirectly, in the case of $\mathrm{BDNF}$ and CTNF for which no receptors are present on photoreceptors, via activation of Müller glia or inner retinal neurons ${ }^{15-17}$. Recently, Müller glial cells were found to be involved in mediating image transfer through the retina by minimizing distortion and loss. Individual Müller glial cells can act as optical fibers and transfer light with low scattering from the retinal surface to the photoreceptor cell layer ${ }^{18}$.

\section{Inherited retinal dystrophies}

Retinal dystrophies in general are a clinically and genetically heterogeneous group of diseases in which degeneration of the retina is progressive, leading to partial or complete blindness. Different mutations in different genes can affect different molecular pathways in the retina, expressed by degeneration at multiple levels. Mutations can affect components of the phototransduction pathway (e.g. rhodopsin, arrestin and PDE6), the visual cycle (e.g. RLBP1 and RPE65), biosynthesis (e.g. ELOVL4 and CYP4V2), eye development (e.g. CRX and NRL), retinal metabolism (e.g. OAT and RGR), cilia maintenance and/or protein transport (e.g. RPGR, RPGRIP1, BBS1-2-4-5-7), synapse functioning (e.g. USH3A, CACNA1F and RIMS1), RNA splicing (e.g. PRPF genes), trafficking of intracellular proteins (e.g. MYO7A and USH1G), phagocytosis (MERTK), apoptosis (e.g. CERKL and CA4), Wnt signaling (e.g. FRD4, LRP5 and NDP) and polarity (e.g. CDH23 and CRB1) ${ }^{19-21}$.

The work described in this thesis focuses on the mammalian Crumbs proteins, of which three homologues have been identified, Crumbs homologue 1 (CRB1), CRB2 and CRB3. Mutations in the Crumbs homologue 1 gene lead to severe retinal dystrophies. Mutations in CRB1 have 
first been identified in individuals with retinitis pigmentosa (RP) type 12 ${ }^{22-24}$. Through a novel suppression subtractive hybridization method, candidate genes that are specifically or preferentially expressed in the human retina were isolated. CRB1 was found to map to chromosome 1q31-q32.1, the region involved in this severe form of autosomal recessive RP with preservation of para-arteriolar retinal pigment epithelium (PPRPE) ${ }^{22}$. CRB1 mutations have also been detected in patients with RP with Coatslike exudative vasculopathy ${ }^{25}$ and early onset RP without PPRPE ${ }^{23,26 .}$

Due to the early onset of symptoms in RP12 patients, CRB1 was considered a good candidate gene for Leber congenital amaurosis (LCA). LCA is the earliest and most severe form of all inherited retinal dystrophies, characterized by severe visual impairment or blindness in the first year of life. LCA accounts for around 5\% of all inherited retinal dystrophies and is one of the main causes of blindness in children ${ }^{27-29}$. Mutations in CRB1 explain $10-15 \%$ of LCA cases ${ }^{25,30-33}$.

In RP, rod photoreceptors are first affected leading to a diminished dark-adapted or scotopic electroretinogram (ERG) response and eventually night blindness. This is followed by progressive death of cone photoreceptors (detected by a diminished light-adapted ERG), restricting the visual field initially in the periphery and in advanced stages often leading to complete loss of vision. RP is estimated to affect one in 4000 persons around the world ${ }^{34,35}$. To date there are no means for prevention or effective (pharmaceutical) treatment, although progress is being made with development of visual neuroprosthesis ${ }^{36}$, identification of neurotrophic factors ${ }^{37}$, transplantation of photoreceptor precursors ${ }^{38}$ and gene therapy approaches ${ }^{39,40}$.

$\mathrm{RP}$ can be inherited in an autosomal dominant, autosomal recessive or X-linked mode. Fifty percent of patients are isolated cases ${ }^{41,42}$. To date, 35 genes have been implicated in non-syndromic RP and 11 loci have been mapped (RetNet, http://www.sph.uth.tmc.edu/Retnet/Table B). Most RP forms are monogenic, but digenic inheritance is also known (reviewed in reference 43). RP patients carrying a heterozygous mutations in the ROM1 gene and in the RDS gene were the first example of digenic-diallelic genetics in human disease 44. Digenic-triallelic inheritance was found in a syndrome, Bardet-Biedl syndrome (BBS), which is characterized by RP, defects in the kidneys and heart, mental retardation and obesity. Some BBS families require two mutant alleles in one gene and one mutant allele in another gene to develop the disease. Other examples in which autosomal recessive RP is presented together with 
additional clinical findings, are Usher syndrome (sensorineural hearing loss), Senior-Løken syndrome (kidney defects, nephronophthisis), Joubert syndrome (primarily neurological features, including psychomotor delay, hypotonia, ataxia and in some cases nephronophthisis) and Refsum disease (heart failure and brain defects) (OMIM http://www.ncbi.nlm. nih.gov/entrez) ${ }^{43,45}$.

\section{Approach to determine the composition and function of the Crumbs protein complex}

At the onset of the work described in this thesis, not much was known about the function of Crumbs proteins in the retina, or about the mechanism by which mutations in the $C R B 1$ gene cause retinal degeneration. It was postulated that the Crumbs protein was involved in maintaining cell polarity in the retina and that the intracellular domain of Crumbs comprises two important protein-binding motifs, for which only one binding partner was identified at that time, namely membrane palmitoylated protein-5 (MPP5). The aim of the work presented here is to determine the composition of the Crumbs protein complex in the mammalian retina, and thereby obtaining clues about its function in health and disease.

In general, by determining binding partners for a protein with an unclear function, more information can be obtained on its cellular function. Furthermore, unraveling the molecular composition of a protein complex in the past has revealed associated genes that are causative for the same or similar diseases. For example, mutations in the RGPR gene (retinitis pigmentosa GTPase regulator) are the main cause of X-linked $\mathrm{RP}^{46,47}$, while mutations in the gene encoding its interacting protein (RPGRIP1) were found in LCA patients ${ }^{48-50}$. Very recently, it was found that RPGRIP1L (KIAA1005), a homologue of RPGRIP1 interacts with nephrocysin-4 (Senior-Løken syndrome). The corresponding gene was mutated in Meckel syndrome and Joubert syndrome type B ${ }^{51,52}$. In Usher syndrome, the most common form of deaf-blindness, eight causative genes that encode proteins of an integrated network have been identified and are found to function in similar pathways in hair cells and photoreceptors (reviewed in reference 53).

To identify novel binding partners of the CRB1 protein complex, yeast two-hybrid screening assays were deployed using bovine or human retinal cDNA libraries. These cDNA libraries contain clones of many genes that are expressed in the retina. Some genes are more abundant 
than others and consequently are more abundantly present in the library. A construct encoding (part of) a protein of interest is used as bait to screen these libraries. This construct is fused to the GAL4 DNA binding domain (BD) that can bind to the yeast GAL4 binding site. The clones present in the library (preys) are fused to the GAL4 activation domain (AD). Both are inserted into the yeast cell by co-transformation or by mating two types of yeast. In the latter method, one mating type of yeast transformed with the bait, mates with the complementary yeast mating type that contains the library. Using this method, more clones containing both bait and prey can be obtained compared to the classical cotransformation method. When bait and library fusion proteins interact, the DNA-BD and AD are brought into close proximity, thus recruiting a RNA polymerase complex and activating transcription of several reporter genes present in the yeast genome (Fig. 5) ${ }^{54,55}$.

Growth on selection plates that lack certain amino acids, and coloring

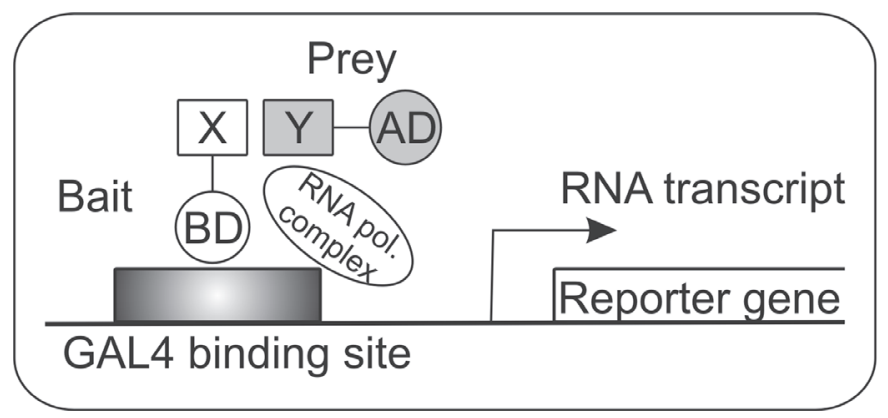

Fig. 5 The principle of a yeast two-hybrid assay (adapted from Stratagene manual HybriZAP system).

in $\alpha$ - and $\beta$-galactosidase reporter assays allow the identification of protein-protein interactions. The level of expression of the reporter genes is a measure for interaction of two proteins. The same assay can be used to test a protein pair for interaction. Upon ascertainment of interaction between two proteins, several complimentary techniques must be performed to determine if the binding is specific. Bacterially expressed fusion proteins in GST-pull down assays or immunoprecipitations from cultured cells can be used to obtain this information. Even more importantly, binding needs to be confirmed in vivo. By combining localization studies in the retina with immunoprecipitations from the same tissues, this information can be gathered. 


\section{Outline of this thesis}

The aim of the work presented in this thesis is to determine the composition and function of the Crumbs protein complex in the mammalian retina. Yeast two-hybrid screens of bovine and human retinal cDNA libraries were performed with the intracellular domain of CRB1 to identify novel interaction partners. These screens solely revealed a known interactor, Membrane Palmitoylated Protein 5 (MPP5 or Pals1) (Chapter 2). The MPP5 protein, belonging to the MAGUK family of proteins, contains many domains for potential protein-protein interactions. Subsequently, screens were performed with those MPP5 domains for which no interacting proteins have been described.

In Chapter 2, the identification of a novel binding partner for MPP5, namely MPP4 is described. MPP4 and MPP5 are found to co-localize in photoreceptors in the retina.

In Chapter 3, yeast two-hybrid screening revealed MPP1 as an additional binding partner for MPP5. Furthermore, MPP1 was found to provide a link between the Usher protein network via interaction with the whirlin protein and the Crumbs protein complex (via MPP5) in the retina.

In Chapter 4, the identification of Syntenin-1 as an interaction partner for MPP5 is described following yeast two-hybrid screening. A function for this protein in the retina is unknown, but its implication in the Crumbs protein complex suggests a role in retinal cell polarity.

Following a candidate binding partner approach, erythrocyte membrane protein band 4.1 like 5 (EPB41L5) is found to be part of the mammalian Crumbs protein complex (Chapter 5). EPB41L5 not only binds to CRB1, CRB2 and CRB3, but also to MPP5. Interestingly, these interactions seem to be conserved throughout evolution.

In Chapter 6, an overview of the composition of the mammalian Crumbs protein complex is given and possible functions of the Crumbs protein complex in the retina are discussed based on data described in this thesis as well as in literature. A more general discussion on the subject is provided in Chapter 7.

Members of the Crumbs protein network are described in literature using the nomenclature as described by the Human Genome Organisation (HUGO) Gene Nomenclature Committee, although sometimes their alias is used. To facilitate literature searches and to make sure that no available data is overlooked, a list of all protein names and their aliases is provided on page 7 . 


\section{REFERENCES}

1. Kandel, E.R., Schwartz J.H. \& Jessell T.M. Essentials of neural science and behaviour. Appleton and Lange, international edition (1995).

2. Dowling, J. E. The Retina - An approachable part of the brain. Cambridge, MA: Harvard University Press (1987).

3. Altshuler, D. M., Turner, D. L. and Cepko, C. L. Specification of cell type in the vertebrate retina. In development of the visual system, pp. 37-58. Cambridge: MIT Press (1991).

4. Boulton, M. \& Dayhaw-Barker, P. The role of the retinal pigment epithelium: topographical variation and ageing changes. Eye 15, 384-389 (2001).

5. Sarna, T. Properties and function of the ocular melanin--a photobiophysical view. J.Photochem.Photobiol.B 12, 215-258 (1992).

6. Rozanowska, M., Sarna, T., Land, E. J., \& Truscott, T. G. Free radical scavenging properties of melanin interaction of eu- and pheo-melanin models with reducing and oxidising radicals. Free Radic. Biol.Med. 26, 518-525 (1999).

7. Korytowski, W., Kalyanaraman, B., Menon, I. A., Sarna, T., \& Sealy, R. C. Reaction of superoxide anions with melanins: electron spin resonance and spin trapping studies. Biochim.Biophys. Acta 882, 145-153 (1986).

8. Osterberg, K. L. Topography of the layer of rods and cones in the human retina. Acta Ophthal., suppl. 6, 1-103 ( 1935).

9. Muniz, A. et al. A novel cone visual cycle in the cone-dominated retina. Exp. Eye Res. 85, 175-184 (2007).

10. Hattar, S. et al. Melanopsin and rodcone photoreceptive systems account for all major accessory visual functions in mice. Nature 424, 76-81 (2003).
11. Panda, S. et al. Melanopsin is required for non-image-forming photic responses in blind mice. Science 301, 525-527 (2003).

12. Bringmann, A. et al. Muller cells in the healthy and diseased retina. Prog. Retin.Eye Res. 25, 397-424 (2006).

13. Tsacopoulos, M. \& Magistretti, P. J. Metabolic coupling between glia and neurons. J.Neurosci. 16, 877-885 (1996).

14. Mata, N. L., Radu, R. A., Clemmons, R. C., \& Travis, G. H. Isomerization and oxidation of vitamin a in cone-dominant retinas: a novel pathway for visual-pigment regeneration in daylight. Neuron 36, 69-80 (2002).

15. Wahlin, K. J., Adler, R., Zack, D. J., \& Campochiaro, P. A. Neurotrophic signaling in normal and degenerating rodent retinas. Exp.Eye Res. 73, 693-701 (2001).

16. Wahlin, K. J., Campochiaro, P. A., Zack, D. J., \& Adler, R. Neurotrophic factors cause activation of intracellular signaling pathways in Muller cells and other cells of the inner retina, but not photoreceptors. Invest.Ophthalmol.Vis. Sci. 41, 927-936 (2000).

17. Kirsch, M., Lee, M. Y., Meyer, V., Wiese, A., \& Hofmann, H. D. Evidence for multiple, local functions of ciliary neurotrophic factor (CNTF) in retinal development: expression of CNTF and its receptors and in vitro effects on target cells. J.Neurochem. 68, 979-990 (1997).

18. Franze, K. et al. Muller cells are living optical fibers in the vertebrate retina. Proc.Natl.Acad.Sci.U.S.A 104, 8287-8292 (2007).

19. Hartong, D. T., Berson, E. L., \& Dryja, T. P. Retinitis pigmentosa. Lancet 368, 1795-1809 (2006).

20. Bolz, H. [Genetics of retinal dystrophies--an overview]. Ophthalmologe 
102, 661-673 (2005).

21. Pierce, E. A. Pathways to photoreceptor cell death in inherited retinal degenerations. Bioessays 23, 605-618 (2001).

22. den Hollander, A. I. et al. Mutations in a human homologue of Drosophila crumbs cause retinitis pigmentosa (RP12). Nat.Genet. 23, 217-221 (1999).

23. Bernal, S. et al. Study of the involvement of the RGR, CRPB1, and CRB1 genes in the pathogenesis of autosomal recessive retinitis pigmentosa. J.Med. Genet. 40, e89 (2003).

24. Khaliq, S. et al. Mutation screening of Pakistani families with congenital eye disorders. Exp.Eye Res. 76, 343-348 (2003).

25. den Hollander, A. I. et al. Leber congenital amaurosis and retinitis pigmentosa with Coats-like exudative vasculopathy are associated with mutations in the crumbs homologue 1 (CRB1) gene. Am.J.Hum.Genet. 69, 198-203 (2001).

26. Lotery, A. J. et al. CRB1 mutations may result in retinitis pigmentosa without para-arteriolar RPE preservation. Ophthalmic Genet. 22, 163-169 (2001).

27. Schappert-Kimmijser, J., HENKES, H. E., \& VAN DEN, B. O. S. C. Amaurosis congenita (Leber). AMA.Arch. Ophthalmol. 61, 211-218 (1959).

28. Kaplan, J., Bonneau, D., Frezal, J., Munnich, A., \& Dufier, J. L. Clinical and genetic heterogeneity in retinitis pigmentosa. Hum.Genet. 85, 635-642 (1990).

29. Perrault, I. et al. Leber congenital amaurosis. Mol.Genet.Metab 68, 200208 (1999).

30. Hanein, S. et al. Leber congenital amaurosis: comprehensive survey of the genetic heterogeneity, refinement of the clinical definition, and genotypephenotype correlations as a strategy for molecular diagnosis. Hum.Mutat. 23,
306-317 (2004).

31. Lotery, A. J. et al. Mutations in the CRB1 gene cause Leber congenital amaurosis. Arch.Ophthalmol. 119, 415-420 (2001).

32. Galvin, J. A., Fishman, G. A., Stone, E. M., \& Koenekoop, R. K. Clinical phenotypes in carriers of Leber congenital amaurosis mutations. Ophthalmology 112, 349-356 (2005).

33. Yzer, S. et al. Microarray-based mutation detection and phenotypic characterization of patients with Leber congenital amaurosis. Invest Ophthalmol.Vis.Sci. 47, 1167-1176 (2006).

34. Boughman, J. A., Conneally, P. M., \& Nance, W. E. Population genetic studies of retinitis pigmentosa. Am.J.Hum. Genet. 32, 223-235 (1980).

35. Bundey, S. \& Crews, S. J. A study of retinitis pigmentosa in the City of Birmingham. II Clinical and genetic heterogeneity. J.Med.Genet. 21, 421-428 (1984).

36. Fernandez, E. et al. Development of a cortical visual neuroprosthesis for the blind: the relevance of neuroplasticity. J.Neural Eng 2, R1-12 (2005).

37. Leveillard, T. et al. Identification and characterization of rod-derived cone viability factor. Nat.Genet. 36, 755-759 (2004).

38. Maclaren, R. E. et al. Retinal repair by transplantation of photoreceptor precursors. Nature 444, 203-207 (2006).

39. Acland, G. M. et al. Gene therapy restores vision in a canine model of childhood blindness. Nat.Genet. 28, 9295 (2001).

40. Alexander, J. J. et al. Restoration of cone vision in a mouse model of achromatopsia. Nat.Med. 13, 685-687 (2007).

41. Fishman, G. A. Retinitis pigmentosa. Genetic percentages. Arch.Ophthalmol. 
96, 822-826 (1978).

42. Wang, Q. et al. Update on the molecular genetics of retinitis pigmentosa. Ophthalmic Genet. 22, 133-154 (2001).

43. Rivolta, C., Sharon, D., DeAngelis, M. M., \& Dryja, T. P. Retinitis pigmentosa and allied diseases: numerous diseases, genes, and inheritance patterns. Hum.Mol.Genet. 11, 1219-1227 (2002).

44. Kajiwara, K., Berson, E. L., \& Dryja, T. P. Digenic retinitis pigmentosa due to mutations at the unlinked peripherin/ RDS and ROM1 loci. Science 264, 1604 1608 (1994).

45. Fleischhauer, J., Njoh, W. A., \& Niemeyer, G. Syndromic retinitis pigmentosa: ERG and phenotypic changes. Klin. Monatsbl.Augenheilkd. 222, 186-190 (2005).

46. Roepman, R. et al. Positional cloning of the gene for X-linked retinitis pigmentosa 3: homology with the guaninenucleotide-exchange factor RCC1. Hum. Mol.Genet. 5, 1035-1041 (1996).

47. Roepman, R. et al. Identification of a gene disrupted by a microdeletion in a patient with X-linked retinitis pigmentosa (XLRP). Hum.Mol.Genet. 5, 827-833 (1996).

48. Roepman, R. et al. The retinitis pigmentosa GTPase regulator (RPGR) interacts with novel transport-like proteins in the outer segments of rod photoreceptors. Hum.Mol.Genet. 9, 2095-2105 (2000).

49. Boylan, J. P. \& Wright, A. F. Identification of a novel protein interacting with RPGR. Hum.Mol.Genet. 9, 2085-2093 (2000).

50. Dryja, T. P. et al. Null RPGRIP1 alleles in patients with Leber congenital amaurosis. Am.J.Hum.Genet. 68, 1295 1298 (2001).

51. Arts, H. H. et al. Mutations in the gene encoding the basal body protein
RPGRIP1L, a nephrocystin-4 interactor, cause Joubert syndrome. Nat.Genet. (2007).

52. Delous, M. et al. The ciliary gene RPGRIP1L is mutated in cerebello-oculo-renal syndrome (Joubert syndrome type B) and Meckel syndrome. Nat. Genet. (2007).

53. Kremer, H., van, W. E., Marker, T., Wolfrum, U., \& Roepman, R. Usher syndrome: molecular links of pathogenesis, proteins and pathways. Hum.Mol.Genet. 15 Spec No 2, R262-R270 (2006).

54. Fields, S. \& Song, O. A novel genetic system to detect protein-protein interactions. Nature 340, 245-246 (1989).

55. Roepman, R., Schick, D., \& Ferreira, P. A. Isolation of retinal proteins that interact with retinitis pigmentosa GTPase regulator by interaction trap screen in yeast. Methods Enzymol. 316, 688-704 (2000). 



\section{CHAPTER 2}

\section{MPP5 recruits MPP4 to the CRB1 complex in photoreceptors}

Albena Kantardzhieva ${ }^{1 *}$, Ilse Gosens ${ }^{2}{ }^{*}$, Svetlana Alexeeva ${ }^{1}$, Ingrid M. Punte ${ }^{2}$, Inge Versteeg ${ }^{1}$, Elmar Krieger ${ }^{3}$, Carla A. Neefjes-Mol ${ }^{1}$, Anneke I. den Hollander ${ }^{2}$, Stef J. F. Letteboer ${ }^{2}$, Jan Klooster ${ }^{1}$, Frans P. M. Cremers ${ }^{2}$, Ronald Roepman ${ }^{2}$ and Jan Wijnholds ${ }^{1}$

${ }^{*}$ Contributed equally to this work and should therefore be considered equivalent authors.

${ }^{1}$ The Netherlands Ophthalmic Research Institute, Meibergdreef 47, 1105 BA, Amsterdam, The Netherlands.

${ }^{2}$ University Medical Centre Nijmegen, Geert Grooteplein Zuid 10, P.O. Box 9101, 6500 HB, Nijmegen, The Netherlands.

${ }^{3}$ Centre for Molecular and Biomolecular Informatics, Toernooiveld 1, P.O. Box 9010, 6500 GL Nijmegen

Published in Investigative Ophthalmology \& Visual Science. (2005) Jun; 46(6):2192-201. 



\section{ABSTRACT}

Purpose. Mutations in the human Crumbs homologue 1 (CRB1) gene are a frequent cause of Leber congenital amaurosis (LCA) and various forms of retinitis pigmentosa. CRB1 is thought to organize an intracellular protein scaffold in the retina that is involved in photoreceptor polarity. This study was focused on the identification, subcellular localization and binding characteristics of a novel member of the protein scaffold connected to CRB1.

Methods. To dissect the protein scaffold connected to CRB1, the yeast two-hybrid approach was used to screen for interacting proteins. Glutathion $S$-transferase (GST) pull-down analysis and immunoprecipitation were used to verify protein-protein interactions. The subcellular localization of the proteins was visualized by immunohistochemistry and confocal microscopy on human retinas and immunoelectron microscopy on mouse retinas.

Results. A novel member of the scaffold connected to CRB1, called membrane palmitoylated protein (MPP) subfamily member 4 (MPP4), a membrane-associated guanylate kinase (MAGUK) protein, was identified. MPP4 was found to exist in a complex with CRB1 through direct interaction with the MPP subfamily member MPP5 (PALS1). 3D homology modeling provided evidence for a mechanism that regulates the recruitment of both homo- and heterodimers of MPP4 and - 5 proteins to the complex. Localization studies in the retina showed that CRB1, MPP5 and MPP4 co-localize at the outer limiting membrane (OLM).

Conclusion. These data imply that MPP4 and -5 have a role in photoreceptor polarity and, by association with CRB1, pinpoint the cognate genes as functional candidate genes for inherited retinopathies. 


\section{INTRODUCTION}

The polarized organization of photoreceptor cells is a fundamental feature of the developing retina. Polarity can be either a dynamic event during which proteins are shuttled across the cell or a more static process in which proteins are clustered in complexes and retained at particular subcellular locations ${ }^{1,2}$. Detailed studies on the factors that play a role in this site-specific localization could provide knowledge about the general pathways that establish and maintain retinal polarity. It can also help us to understand the pathological pathways in the retina that are triggered by mutations in genes that encode components of such complexes.

Several members of the membrane associated guanylate kinase (MAGUK) protein family are involved in cell polarity through their role in large multiprotein complexes at tight junctions ${ }^{3,4}$. This protein family is characterized by a specific set of protein-binding domains, consisting of one or more PDZ (postsynaptic density 95/discs large/zonula occludens 1) domains, an SH3 (Src homology 3) domain, and a region with homology to a guanylate kinase (GUK) domain ${ }^{5,6}$. Some members, such as PALS1 (assigned the name MPP5 by the HUGO Gene Nomenclature Committee; gene.ucl.ac.uk/nomenclature; hosted by the University College London, London, UK), ZO1 and PSD-95, were shown to be localized at sites of cell-cell contact (e.g., synapses and epithelial tight junctions) ${ }^{7-10}$. It is currently assumed that MAGUK proteins serve as scaffolds by recruitment of other MAGUKs, eventually linking them to the cell cytoskeleton or to the carboxyl-terminus of transmembrane proteins ${ }^{4,11,12}$.

The MAGUK protein Stardust is the Drosophila homologue of MPP5, one of the seven mammalian membrane palmitoylated protein (MPP) subfamily members. Stardust mutants exhibit severe disruption in apicobasal polarity of embryonic epithelia ${ }^{13}$. In addition, mutants of the zebra fish homologue Nagie oko display severe defects in the organization of the retinal cell layers, ${ }^{14}$ and loss of Stardust gives rise to an eye phenotype in Drosophila characterized by a shortened stalk membrane and altered rhabdomere morphogenesis resembling the Crumbs mutant phenotype ${ }^{15-17}$.

Crumbs is an apically localized transmembrane protein involved in organizing the apical plasma membrane subdomains ${ }^{18-21}$. Stardust has been found to colocalize with Crumbs and interact physically with the C-terminal ERLI motif of Crumbs via its PDZ domain ${ }^{22}$. This interaction was recently also identified for their mammalian homologues MPP5 and $\mathrm{CRB} 1{ }^{10}$ as well as for MPP5 and CRB3 ${ }^{23}$. Stardust and Crumbs are 
both necessary to ensure stability, localization, and function in controlling the apicobasal polarity of epithelial cells ${ }^{18,22}$. The Crumbs-Stardust protein complex also recruits the Drosophila protein associated with tight junctions (DPATJ) (DPATJ; formerly known as Discs lost). This cytoplasmic multi-PDZ domain protein interacts indirectly, via Stardust, with the cytoplasmic tail of Crumbs. These three proteins colocalize in Drosophila photoreceptors during and after eye development ${ }^{17}$. The mammalian CRB1-MPP5-PATJ complex localizes to tight junctions where it may control cell polarity ${ }^{10}$. In the mouse retina, the CRB1-MPP5-PATJ proteins co-localize at the apical region adjacent to adherens junctions of photoreceptors ${ }^{24}$. The Drosophila Crumbs protein and the human homologue CRB1 are 35 percent similar in amino acid sequence and contain the same conserved protein motifs ${ }^{25}$. Mutations have been identified in the CRB1 gene in individuals with Leber congenital amaurosis (LCA); retinitis pigmentosa (RP) type 12, with preservation of para-arteriolar retinal pigment epithelium (PPRPE); RP with Coats-like exudative vasculopathy and early onset RP without PPRPE ${ }^{26-29}$. CRB1 has been found to maintain adherens junctions between photoreceptor cells and Müller glia cells ${ }^{30}$, thus preventing delamination of the photoreceptor layer and death of retinal neurons ${ }^{24,31}$.

In this study we identified a second MPP subfamily member, MPP4, existing in a complex with CRB1 through direct interaction with MPP5. The presence of MPP4 and -5 in this CRB1 protein complex implicates these proteins in photoreceptor polarity and putatively in inherited retinal dystrophies.

\section{MATERIALS AND METHODS}

\section{DNA constructs}

Human retinal cDNA (Marathon Ready; BD-Clontech, Palo Alto, CA) or mouse $\mathrm{B} 6 \mathrm{D} 2$ retinal cDNA synthesized with the Marathon cDNA amplification Kit (Marathon; BD-Clontech), was used to amplify the fulllength cDNAs for human CRB1, MPP5, MPP4, and mouse Mpp4 with a cDNA PCR Kit (Advantage; Clontech). For human CRB1 the following primers were used: 5'- GGGATCCAAATACCACC ATGGCACTTAAGAACATTAACTAC-3' (sense) and 5'-GATCCTCGAGTCCTAAATCAGTCTCTCCATTGCAGG-3' (antisense). Italic sequences denote start and stop codons of the gene. Two consecutive Myc tags were inserted at amino acid (aa) position 1331 with the following primers: 5'- 
GCGAACAAAAACTCATCTCAGAAGAGGATCTG-3' (sense), and 5'-GCAGATCCTCTTCTGAGATGAGTTTTTGTTC-3' (antisense). The human MPP5 PCR was performed with primers: 5'-GATCCCGGGCCATCATGACAACATCCCATATGAATGGGCATG-3' (sense) and 5'-GATCGTCGACTCACCTCAGCCAAGTGGATGGTAC-3' (antisense). Human MPP4 was synthesized with primers: 5'-GATCCCGGGCCATCATGATACAGTCAGACAAAGGAGCAG-3' (sense) and 5'-GATCGTCGACTCATTGAGACTCAGTATCTGAG-3' (antisense), and mouse Mpp4 with: 5'-GATCCCGGGCCATCATGAGACAGTCTGACAGAGGAGCAG-3' (sense) and 5'-GATCGTCGACTTAAGATTCAGTGTCAGGGGAAACC-3' (antisense). A 3xFLAG epitope tag was created at the N-terminus of human MPP4 with the following primers: 5'-GACTACAAAGACCATGACGGTGATTATAAAGATCATGACATCG-ATTACAAGGATGACGATGACAAGCTCATG-3' (sense), and 5'- GTACAGCTTGTCATCGTCATCCTTGTAATCGATGTCATGATCTTTATAATCACCGTCATGGTCTTTGTAGTC - -3’ (antisense).

The following constructs were made by PCR using a cloning system (Gateway; Invitrogen, Groningen, the Netherlands), according to the manufacturer's procedures, using full-length constructs as a template: the intracellular domains of bovine CRB1 (bCRB1 ${ }^{\text {intra }}$ ) and human CRB1 (hCRB1 ${ }^{\text {intra }}$ ); full length human MPP5 (MPP5 $^{\mathrm{FL}}$ ); the $\mathrm{SH} 3$ and HOOK domain of human MPP5 (MPP5 $\left.{ }^{\mathrm{SH} 3+\mathrm{HK}}\right)$; the C-terminus of human MPP5 from the HOOK domain (MPP5 $\left.{ }^{\mathrm{HK}-\text { end }}\right)$; the PDZ domain of human MPP5 (MPP5 ${ }^{\mathrm{PDZ}}$ ); the coiled-coil domain of human MPP5 (MPP5 ${ }^{\mathrm{CC}}$ ); the GUK domain of human MPP5 (MPP5 ${ }^{\mathrm{GuK}}$ ); full length human MPP4 $\left(\mathrm{MPP}^{\mathrm{FL}}\right)$; the PDZ domain of human MPP4 (MPP4 ${ }^{\mathrm{PDZ}}$ ); the C-terminus of MPP4 containing the E-domain and GUK domain, (MPP4 ${ }^{\text {Edom-end }}$ ); the C-terminus of MPP4 (MPP4 ${ }^{365-637}$ ); the SH3 and HOOK domain of human MPP4 (MPP4 ${ }^{\mathrm{SH} 3+\mathrm{HK}}$ ); the C-terminus of MPP4 from the $\mathrm{SH} 3$ domain (MPP4 $\left.{ }^{\text {SH3-end }}\right)$. Gene-specific primers that were used to make these constructs are listed in Table 1. The attB1 and attB2 linkers were attached to the 5 ' end of, respectively, sense and antisense primers.

The commercially adapted destination vectors pBD-GAL4/DEST and pAD-GAL4/DEST (Gateway; Invitrogen) were created by insertion of the blunt-ended reading frame (Rf) cassette B into, respectively, the EcoRI and SalI sites of pBD-GAL4-2.1-Cam (Stratagene, Amsterdam) and the BamHI and SalI sites of pAD-GAL4-2.1 (Strata- 
gene) ${ }^{32}$, with sticky ends previously filled in using Klenow (Invitrogen). The destination vectors pDest-15 (N-GST fusion tag) and pDest-17 (N-6xHis fusion tag) were purchased from Invitrogen.

Table 1. Sense and antisense primer sequences for Gateway constructs

\begin{tabular}{|c|c|c|}
\hline Construct (a.a. position) & Sense & Antisense \\
\hline bCRB $1^{\text {intra }}(1372-1408)$ & 5'-TCGCCTCCAACAAAAGGGCAAC-3' & 5'-CCTAGATCAGCCTCTCTGCTGCAG-3', \\
\hline hCRB $1^{\text {intra }}(1369-1406)$ & 5'-TCACСТCCAACAAAAGGGCAACTCA-3' & 5'-CCTAAATCAGTCTCTCCATTGC-3' \\
\hline $\mathrm{hMPP}^{\mathrm{FL}}(1-675)$ & 5'-ATGACAACATCCCATATGAAT-3' & 5'-TCACCTCAGCCAAGTGGATGG-3' \\
\hline hMPP5 $^{\text {SH3+HK }}(377-477)$ & 5'-CAGATCAAGCCGCCTCCTG-3' & 5'-СTTCCTATTTGCTGGCTGATG -3' \\
\hline $\mathrm{hMPP}^{\mathrm{PDZ}}(237-348)$ & 5'-TTACAGATGAGAGAGTTTATGAAAG-3' & 5'-GGATTACTGTTTCCTTGGCAGG-3' \\
\hline hMPP5 $^{\mathrm{CC}}(1-122)$ & 5'-ATGACAACATCCCATATGAATG-3', & 5'-TAATATTTTCACAGCATAATGAGG-3 \\
\hline hMPP5 $5^{\mathrm{GuKc}}(470-675)$ & 5'-CTTTATCATCAGCCAGCAAATAGG-3' & 5'-TCACCTCAGCCAAGTGGATGG-3' \\
\hline $\mathrm{hMPP}^{\mathrm{HK}-\mathrm{end}}(408-675)$ & 5'-CCAGGGAAAAGCTTTCAGCAGC-3' & 5'-TCACCTCAGCCAAGTGGATGG-3' \\
\hline $\mathrm{hMPP} 4^{\mathrm{FL}}(1-637)$ & 5'- ATGATACAGTCAGACAAAGGAG-3' & 5'-TCATTGAGACTCAGTATCTG-3' \\
\hline $\mathrm{hMPP} 4^{\mathrm{SH} 3+\mathrm{HK}}(245-425)$ & 5'-GTGTACGTCCGTGCCATGAC-3' & 5'-GTCTATGACTCAGAGTTACT-3' \\
\hline $\mathrm{hMPP} 4^{\mathrm{PDZ}}(141-244)$ & 5'-TGCCAGACAATATCCCTGAGAG-3' & 5'-CCATCTGCTGGCTATTCACAGG-3' \\
\hline hMPP4 ${ }^{\text {SH3-end }}(245-637)$ & 5'-GTGTACGTCCGTGCCATGAC-3' & 5'-TCATTGAGACTCAGTATCTG-3' \\
\hline hMPP4 ${ }^{\text {Edom-end }}$ (410-637) & 5'-GGTGCCCCTTACGAGGAGG-3' & 5'-TCATTGAGACTCAGTATCTG-3' \\
\hline hMPP4 $4^{365-637}(365-637)$ & 5'-GAGGAGTTTGTTGGCTACGG-3' & 5'-TCATTGAGACTCAGTATCTG-3' \\
\hline
\end{tabular}

The pDest566 was constructed by introduction of a reading frame cassette (Gateway; Invitrogen) into a modified version of pET-43a (Novagen, Madison, WI) containing an amino terminal His6-maltose-binding protein tag (Esposito D. , Hartley J, unpublished data, 2003). All novel constructs were verified by nucleotide sequencing.

\section{Yeast two-hybrid}

A GAL4-based yeast two-hybrid system (Hybrizap; Stratagene), with yeast strain PJ69-4 $\alpha$, was used to identify proteins that interact with $\mathrm{CRB}^{\text {intra }}$ and MPP5. The pBD-hMPP5 ${ }^{\mathrm{CC}}, \mathrm{pBD}-\mathrm{hMPP5}{ }^{\mathrm{GuK}}$ and pBD$\mathrm{hMPP} 5^{\mathrm{SH} 3+\mathrm{HK}}$ constructs were used as baits on an oligo-dT primed human retina cDNA library, representing $2.1 \times 10^{6}$ primary cDNA clones. In total, $8.2 \times 10^{5}, 1 \times 10^{6}$ and $6.8 \times 10^{5}$ clones were plated, respectively. The human and bovine $\mathrm{pBD}-\mathrm{CRB} 1^{\text {intra }}$ constructs were used to screen respectively a pretransformed oligo-dT primed human, and a randomly primed bovine retina cDNA library by yeast cell-to-cell mating resulting in screening $10 \times 10^{6}$ and $14 \times 10^{6}$ clones $^{33}$. In subsequent yeast two-hybrid experiments, different domains of MPP5 and -4 were tested for interaction by cotransformation into the YRG-2 yeast strain. Interactions were quantified in a liquid ortho-nitrophenyl- $\beta$-D-galactopyranoside (ONPG) assay for $\beta$-galactosidase activity ${ }^{34}$. 


\section{Antibodies}

Bacterially expressed full-length MPP5 protein was used for immunization of chicken. The yolk was processed with an IgY Purification Kit (Eggcelent Chicken; Pierce Biotechnology, Rockford, IL) according to the manufacturer's protocol, and IgY antibodies were consequently affinity purified on a protein-coupled NHS-activated HP column (Hi-Trap; Amersham Biosciences, Roosendaal, The Netherlands).

Production AK2, AK5, AK7, AK4 and AK8 antibodies have been described ${ }^{24}$. Anti-c-myc monoclonal mouse antibodies (clone 9E10) were purchased from Roche, anti-rat MUPP1 (clone 43) and anti- $\beta$-catenin (clone 14) mouse monoclonal antibodies from BD Biosciences (Leiden, The Netherlands), anti-6x His antibody from Santa Cruz (Heerhugowaard, The Netherlands), anti-FLAG monoclonal mouse antibody (clone M2), monoclonal anti-chicken IgG (clone CG-106) and rat monoclonal antiuvomorulin (clone Decma-1) from Sigma-Aldrich (Amsterdam, The Netherlands). Secondary antibodies conjugated to Alexa 488, Cy3, and Cy5 were obtained from Molecular probes (Leiden, The Netherlands and Jackson ImmunoResearch Laboratories (West Grove, PA). Secondary antibodies conjugated to horseradish peroxidase, were purchased from Sigma-Aldrich and Zymed (Uden, The Netherlands).

\section{Cell culture}

Human embryonic kidney (293HEK) and Madin-Darby canine kidney type II (MDCKII) cells were grown in DMEM (Invitrogen) containing $1 \%$ penicillin/streptomycin and 10\% fetal bovine serum.

Stably transfected MDCKII/CRB1 clones were generated by transduction of MDCKII cells with pBABE-CMV-Puro-CRB1 retroviruses, and subsequently selected with $2 \mu \mathrm{g} / \mathrm{ml}$ Puromycin.

\section{GST pull-down, Coimmunoprecipitation, Western blot analysis} Arabinose inducible BL21-AI cells were transformed with GST-hCRB1 ${ }^{\text {in }}$ tra / pest 15 or His-MBP-hMPP5 ${ }^{\mathrm{PDZ}} / \mathrm{pDest566}$ and IPTG inducible BL21DE3 cells with GST-hMPP5 ${ }^{\mathrm{SH} 3+\mathrm{HK}} / \mathrm{pDest}^{15}$ or His-MBP-hMMP4 ${ }^{\mathrm{HK}}$ end $/$ pDest566. BL21-DE3 cell lysates were prepared according to a $1.5 \%$ sarkosyl protocol with DNAse added before centrifugation ${ }^{35}$. BL21-AI cell lysates were prepared (B-Per; Pierce Biotechnology, Etten-Leur, The Netherlands) with protease inhibitors cocktail (Roche, Almere, The Netherlands) plus $1 \mu \mathrm{g} / \mathrm{ml}$ pepstatin A and $5 \mathrm{mM} \mathrm{DTT}$. For GST pull downs, equal amounts of blocked $(1.5 \mathrm{mg} / \mathrm{ml} \mathrm{BSA})$ glutathione Sepharose 
beads (4B; Amersham Pharmacia, Uppsala, Sweden) with glutathione $S$ transferase (GST), beads with GST fusion proteins or beads alone were incubated with $0.5 \mathrm{ml}$ of lysates containing His-MBP-fusion proteins for 2 hours at $4^{\circ} \mathrm{C}$. After several washes with lysis buffer and TBS containing 1\% triton X-100 and $2 \mathrm{mM}$ DTT, the beads were boiled and proteins were resolved on SDS-polycrylamide gels.

For coimmunoprecipitation experiments, 293HEK cells were transfected with pBabe-CMV-Puro/Hygro-CRB1/MPP5/MPP4 with a commercial reagent (Fugene 6; Roche) or calcium phosphate. After 48 hours, cells were lysed in $50 \mathrm{mM}$ HEPES ( $\mathrm{pH}$ 7.4), $150 \mathrm{mM}$ sodium chloride, $10 \%$ glycerol, 0.5\% Triton X-100, $1.5 \mathrm{mM}$ magnesium chloride, $1 \mathrm{mM}$ EGTA, $1 \mathrm{mM}$ phenylmethylsulfonyl fluoride (PMSF), a protease inhibitor cocktail (Roche) and $10 \mu \mathrm{g} \mathrm{ml}^{-1}$ aprotinin (Sigma-Aldrich). Either Protein LAagarose (Sigma-Aldrich) was used to bind the primary antibodies after incubation of precleared supernatants with 10 to $15 \mu \mathrm{g}$ antibody for 4 to 16 hours at $4^{\circ} \mathrm{C}$, or antibodies were precoupled to protein $\mathrm{G}$ beads (Dynabeads, Dynal Biotech ASA, Oslo, Norway) before incubation of supernatants for 2 hours at $4^{\circ} \mathrm{C}$. For immunoprecipitations with anti-MPP5 antibody SN47, mouse monoclonal anti-chicken IgG was precoupled to protein $\mathrm{G}$ Dynabeads (15 $\mu \mathrm{g} /$ reaction), followed by a second round of coupling of chicken anti-MPP5 antibody SN47 (10 $\mu \mathrm{g} / \mathrm{reaction})$ and incubation with cell lysates for 2 hours at $4^{\circ} \mathrm{C}$. The beads were washed three times in 10\% glycerol/PBS or lysis buffer, respectively and boiled in sample buffer with $\beta$-mercaptoethanol, and the immunocomplexes were resolved by SDS-PAGE. For Western blot analyses, proteins were electrophoretically transferred onto nitrocellulose membranes, which were then blocked, incubated with primary and secondary antibodies (conjugated to horseradish peroxidase) in $0.3-5 \%$ milk powder/TBS, and washed in TBS. The bands were visualized with a chemiluminescence reagent (ECL; Amersham Biosciences).

\section{N-glycosylation experiments}

After reaching $70 \%$ confluence, stable clones of MDCKII cells expressing hCRB1 were cultured for 1-5 days in DMEM supplemented with 1\% penicillin/streptomycin, $10 \%$ fetal bovine serum and $5 \mu \mathrm{g} / \mathrm{ml}$ tunicamycin dissolved in dimethylsulfoxide (DMSO) or only DMSO for the control cells. 


\section{Expression profiles}

Total RNA was isolated from different human tissues and from an ARPE-19 cell-line ${ }^{36}$. For the semiquantitative RT-PCR, 3.1 $\mu \mathrm{g}$ RNA was reverse transcribed using random hexanucleotides ${ }^{37}$. A touchdown PCR was performed for 28 and 33 cycles on $62 \mathrm{ng}$ cDNA for CRB1, MPP5, MPP4 and the housekeeping gene GAPDH, which served as a standard. The following primer pairs were used: 5'ACCAATGTATTCAACAGGGACC-3'(sense) and 5'-TCGTTTCCGTTGTAGTGTCTCC3' (antisense) for CRB1, 5'- GTATGGAACTAGCATAGATTCTG-3' (sense) and 5'- CAAGATCGGAATTCACAATTGCC-3' (antisense) for MPP5, 5'-CACCTGTATGGCACTAGTGTGG-3'(sense) and 5'-CATACACCTCATATTCGATGGC-3'(antisense) for MPP4, 5'-ACCACAGTCCATGCCATCAC-3' (sense) and 5'-TCCACCACCCTGTTGCTGTA3'(antisense) for GAPDH.

\section{Immunohistochemistry}

Eight human postmortem retinas, with enucleation times of 8 to 24 hours, were obtained from the cornea bank in Amsterdam and treated in accordance with the Declaration of Helsinki for the use of human tissue in research.

Frozen human retina sections, $10 \mu \mathrm{m}$ thick, were treated essentially as described previously ${ }^{24}$ using PBS buffer and 1\% BSA. Sections were imaged on a confocal laser-scanning microscope (model 501; Carl Zeiss Meditec, Jena, Germany).

\section{Immunoelectron Microscopy}

Immunoelectron microscopy on mouse retina sections was performed as described previously ${ }^{38}$. Ultra thin sections were examined and photographed (model 201 electron microscope; Phillips, Eindhoven, The Netherlands).

\section{Molecular modeling of MPP4 and -5}

The amino acid sequences of MPP4 (Swiss Prot accession number Q96JB8; http://www.expasy.org; provided in the public domain by Swiss Institute of Bioinformatics, Geneva, Switserland) and MPP5 (accession number Q8N3R9) were submitted to the 3D-PSSM fold recognition server ${ }^{39}$ to search the protein data base (PDB) for homology modeling templates. The best hit in both cases (E-value 0.07) was PDB file 1KJW, the SH3GUK module of Postsynaptic Density Protein 95 (PSD-95), solved at 
$1.8 \AA$ resolution ${ }^{40}$. With $\sim 40 \%$ sequence identity in the aligned regions, the modeling template PSD-95 can be expected to be very similar to the target structures ${ }^{41}$, except for structurally divergent loop regions. Consequently, homology models for MPP4 and -5 were built with WHAT IF ${ }^{42}$, using a backbone-dependent rotamer library ${ }^{43}$ (see Fig. 3B, left side). Flexible HOOK residues (blue in Fig. 3B) were deleted, and the independent $\mathrm{SH} 3$ and GUK domains of MPP4 and -5 were arranged in all four possible permutations. Finally the side-chain rotamers at the domain interface were optimized with YASARA (Yasara Biosciences, http://www. yasara.org/index.html) by minimizing the NOVA force field energy ${ }^{44}$. The parameters of the NOVA energy function have been optimized based on known high-resolution x-ray structures, so that the function has stable minima as close as possible to these structures. The relative domain-binding energies of the four models were then calculated as described previously ${ }^{45}$. Coordinate files of the models are available from the authors upon request.

\section{RESULTS}

\section{Screening for interaction partners of $\mathrm{CRB} 1^{\text {intra }}$}

The conserved putative PDZ and FERM protein-binding regions in the intracellular domain of CRB1 suggest target epitopes for different interactions in this relatively small domain. Therefore, we used this domain as a bait to screen bovine and human yeast two-hybrid retina cDNA libraries. We identified only MPP5 as an interacting protein from the bovine randomly primed library library (13 clones out of $1.4 \times 10^{7} \mathrm{co}-$ transformants analyzed). A highly saturated screen of the human oligodT-primed retina cDNA library $\left(1 \times 10^{7}\right.$ cotransformants) did not reveal any interactors.

\section{Identification of a novel interactor of MPP5}

Although the MAGUK proteins in general, and the MPP family of proteins in particular, contain different conserved putative protein-protein interaction domains that allow the build up of a scaffold, only a few of these domains in MPP5 have known ligands (Fig. 1). We used the conserved epitopes of human MPP5, for which no partners have yet been identified, as baits in yeast two-hybrid screens of a human oligodT-primed retina cDNA library. We did not identify interactors for the coiled-coil domain (amino acid [aa] 1-122) nor for the GUK domain (aa 
470-675; data not shown). However, a bait construct containing the SH3HOOK region of human MPP5 was found to interact with MPP4. Four different clones containing the C-terminus of MPP4 were identified, with the GUK domain flanked by strands $\mathrm{E}$ and F (Fig. 1), starting at aa. 319 (\#228), aa. 365 (\#73), aa. 368 (\#221) and aa. 390 (\#78) (Fig. 2A). The binding affinities of these different clones for MPP5 were measured semi-quantitatively in a liquid ortho-nitrophenyl- $\beta$-D-galactopyranoside (ONPG) assay, revealing that the peptide stretch of MPP4 containing the region $\mathrm{E}, \mathrm{GUK}$ and $\mathrm{F}$ is essential for the interaction. However, when more amino acids are present at the $\mathrm{N}$-terminus, the binding affinity increased.

D.m. Stardust

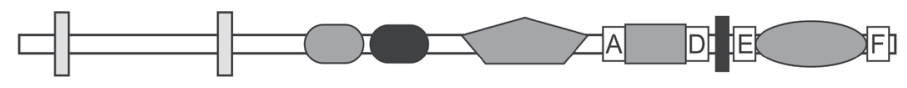

H.s. MPP5

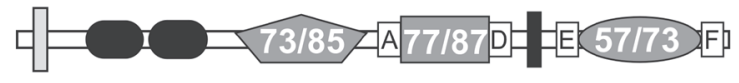

H.s. MPP4

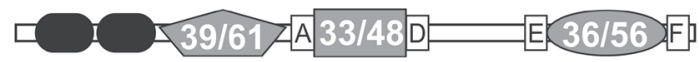

M.m. Mpp4
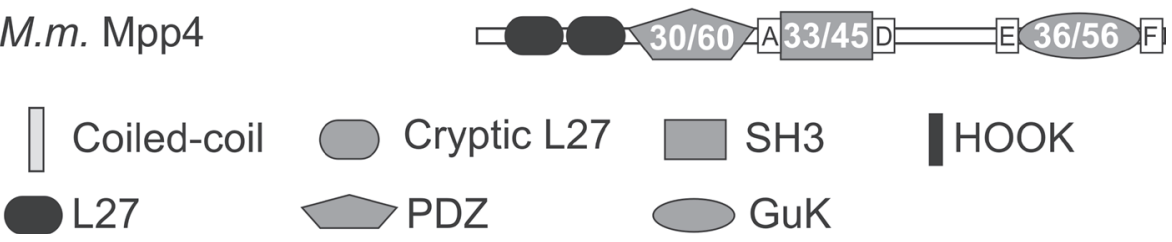

Fig.1 Alignment of Stardust homologues

Stardust was individually aligned with its closest human homologue MPP5 and with human and mouse MPP4. The percentages of identical and similar amino acid sequence of the conserved PDZ, SH3 and GUK domains are shown in the respective boxes. Stardust and MPP5 contain an additional HOOK domain. Strands A and D flanking the SH3 domain and $\mathrm{E}$ and $\mathrm{F}$ flanking the GUK domain were identified according to homology with PSD-95.

\section{Association of MPP4 and -5 In Vitro and In Vivo}

The interaction between MPP4 and -5 was confirmed in a GST pulldown assay (Fig. 2B). GST-MPP5 ${ }^{\mathrm{SH} 3+\mathrm{HK}}$ fused to glutathione-Sepharose efficiently pulled down His-MBP-MPP4 ${ }^{365-637}$ (Fig. 2B).

Furthermore, to test for a physical interaction between MPP4 and -5 in the presence or absence of CRB1, we used 293HEK cells overexpressing MPP4, and/or MPP5, and/or CRB1 in immunoprecipitation experiments (Fig. 2C-D). Anti-FLAG antibody coimmunoprecipitated MPP5 
from cells overproducing 3xFLAG-MPP4 and MPP5, as well as from cells overproducing 3x FLAG-MPP4, MPP5 and CRB1 (Fig. 2C). The 80 $\mathrm{kDa}$ recombinant and the endogenously expressed $70 \mathrm{kDa}$ MPP5 coimmunoprecipitated with MPP4 (Fig. 2C). In a reciprocal experiment, antiMPP5 antibody SN47 coimmunoprecipitated MPP4 from cells overproducing 3xFLAG-MPP4 and MPP5, as well as from cells overproducing 3x FLAG-MPP4, MPP5 and CRB1 (Fig. 2D). Coimmunoprecipitation of MPP4 or CRB1 with immunoprecipitated endogenous 70 - and $80-\mathrm{kDa}$ MPP5 was below detection levels (Fig. 2D, and data not shown). Recombinant MPP5 was efficiently immunoprecipitated by SN47 from cell lysates overproducing MPP5 whereas endogenous MPP5 was not (data not shown). By Western blot analysis we could not detect endogenous expression of CRB1 (data not shown). These results show that MPP4 interacts with MPP5 in the absence as well as presence of CRB1. To test for the presence of a protein complex containing MPP4 and CRB1, we used 293HEK cells overexpressing 3xFLAG-MPP4 and/or myc-CRB1. Anti-MPP4 antibody (AK4) immunoprecipitated MPP4 from 3xFLAGMPP4 overproducing cell lines (Fig. 2E), and coimmunoprecipitated CRB1 from cells overproducing 3xFLAG-MPP4 and myc-CRB1 (Fig. 2F), though at a much lower level.

In a reciprocal experiment, anti-myc antibody immunoprecipitated CRB1 from myc-CRB1 overproducing cell lines (data not shown), and coimmunoprecipitated MPP4 from cells overproducing 3xFLAG-MPP4 and myc-CRB1 at similar low levels (Fig. 2G). This result, as well as the anti-flag immunoprecipitation of MPP4, indicates the presence of MPP4 and CRB1 in the same complex, likely through endogenously expressed MPP5. The calculated size of CRB1 was $154 \mathrm{kDa}$, while CRB1 polyclonal antibodies recognized a protein $>220 \mathrm{kDa}$ on Western blot from 293HEK/CRB1 and MDCKII/CRB1 cells. The PROSITE program predicted 23 putative N-glycosylation sites (Swiss Institute of Bioinformatics). Therefore, we tested whether CRB1 is glycosylated in MDCKII/ CRB1 cells. Incubation with tunicamycin shifted the molecular weight towards the expected $154 \mathrm{kDa}$ (Fig. $2 \mathrm{H}$ ), indicating extensive N-glycosylation of CRB1. The endogenous Crb1 from mouse retina ${ }^{24}$ appears to be over $220 \mathrm{kDa}$, while the calculated molecular weight is $153 \mathrm{kDa}$. This suggests that $\mathrm{N}$-glycosylation also occurs in vivo in mouse retina. 
A MPP5
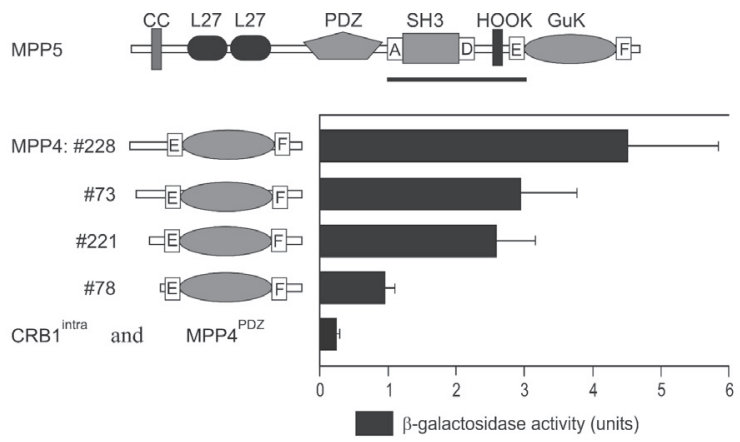

B

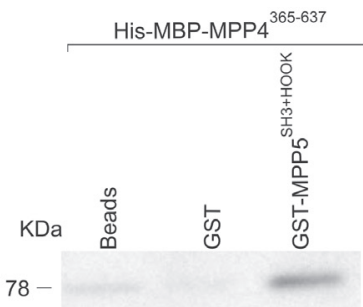

Blot: anti-His

c

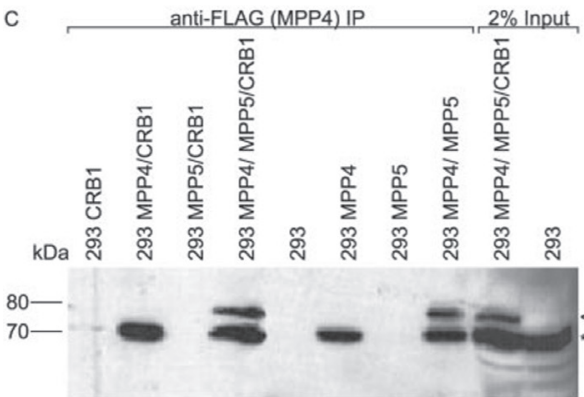

Blot anti-MPP5

E
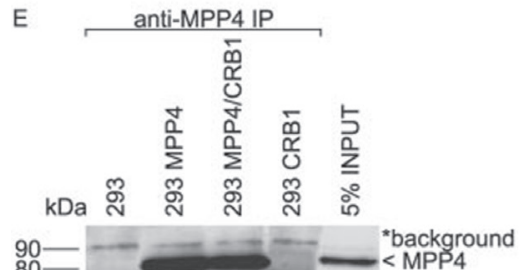

Blot anti-MPP4

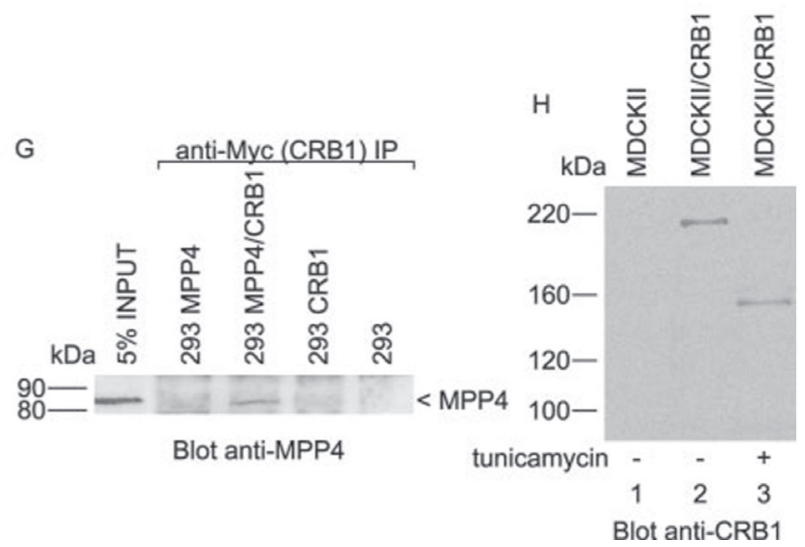


Fig. 2 Identification and confirmation of interactions between MPP5 and MPP4.

(A). Determination of the relative binding affinity between MPP5 and -4. YRG2 yeast cells were cotransformed with the bait (SH3-HOOK region of MPP5) and different clones of MPP4 from the yeast two-hybrid screen. Activation of the $L a c Z$ reporter gene was determined by a liquid ONPG assay ( $\beta$-galactosidase activity, indicated by the black bars). In this assay, the pBD-GAL4 domain fused to the intracellular domain of CRB1, which has no binding affinity for the PDZ domain of MPP4, was used as a negative control showing basal levels of reporter gene activation.

(B). Interaction between MPP5 and MPP4 was confirmed in a GST-MPP5 $5^{\mathrm{SH} 3+\mathrm{HK}}$ pull down from bacterial lysates expressing His-MBP-MPP4 ${ }^{365-637}$.

(C). Anti-FLAG antibody coimmunoprecipitated MPP5 from cells overproducing 3xFLAG-MPP4 and MPP5, as well as from cells overproducing 3xFLAGMPP4, MPP5 and CRB1-myc. Asterisk indicates an endogenous $70 \mathrm{kDa}$ form of MPP5, co-immunoprecipitated from all 3xFLAG-MPP4 overexpressing cells.

(D). Anti-MPP5 antibody (SN47) coimmunoprecipitated MPP4 from cells overproducing 3xFLAG-MPP4 and MPP5, as well as from cells overproducing 3x FLAG-MPP4, MPP5 and CRB1-myc.

(E). The anti-MPP4 antibody (AK4) immunoprecipitated MPP4 from cells overproducing MPP4 or CRB1-myc and MPP4. Asterisk indicates an unspecific band of $92 \mathrm{kDa}$.

(F). Anti-MPP4 antibody (AK4) coimmunoprecipitated CRB1-myc from cells overproducing CRB1-myc and MPP4.

(G). Anti-myc antibody coimmunoprecipitated MPP4 from cells overproducing CRB1-myc and MPP4.

$(\mathrm{H})$. N-glycosylation of CRB1. Incubation of stable MDCKII/CRB1 cells with tunicamycin shifted the molecular weight from over $220 \mathrm{kDa}$ towards the calculated $154 \mathrm{kDa}$, indicating extensive N-glycosylation of CRB1.

\section{Computer-Based Molecular dynamics and in vivo interaction of homo-and heterodimers of MPP4 and -5}

The interacting domains of MPP4 and -5 were analyzed by using a panel of deletion variants of both proteins in the yeast two-hybrid system (Fig. $3 \mathrm{~A}$, above dotted line). A fragment of MPP5 containing the SH3-HOOK region, flanked by strands $\mathrm{A}$ and $\mathrm{D}$, specifically interacts with a fragment of MPP4 containing the GUK domain, flanked by strands E and F. When either of these strands was absent, the interaction was fully disrupted (data not shown). Furthermore, no interaction was found between the SH3-HOOK region of MPP5 and the full-size SH3-GUK module of MPP4, or between the full length MPP5 and -4 proteins (data not shown). These results are analogous to the intra- and intermolecular interactions 
reported for the MAGUK protein PSD-95. In PSD-95, the interaction between the SH3 and GUK domains can either occur within a single peptide chain or between separate peptide chains. It has been proposed before that the interactions observed in PSD-95 are a conserved feature among MAGUK proteins ${ }^{46}$. A similar mechanism could thus be expected for MPP4/MPP5 based on our experimental results and the high percentage of sequence identity to PSD-95, especially at the domain interface. In order to analyze this possibility, we built 3D homology models of the SH3-GUK domains of MPP4 and -5 (Fig. 3B, left), based on the crystal structures of PSD-95 ${ }^{40,46}$. All four permutations of domain interactions were analyzed: $\mathrm{MPP}^{\mathrm{SH}}{ }^{\mathrm{S}}-\mathrm{MPP} 4^{\mathrm{GuK}}, \mathrm{MPP}^{\mathrm{SH}}{ }^{\mathrm{S}}-\mathrm{MPP}^{\mathrm{GuK}}, \mathrm{MPP}^{\mathrm{SH}}-\mathrm{MP}-$ $\mathrm{P} 4^{\mathrm{GuK}}$ and $\mathrm{MPP}^{\mathrm{SH}}{ }^{3}-\mathrm{MPP} 5^{\mathrm{GuK}}$. In all cases, the domain interfaces were stabilized by salt bridges and hydrophobic interactions, most prominently by a conserved Tyrosine (Y413 in MPP4 and Y466 in MPP5) that forms the core of the SH3 domain, but belongs to the E-strand just before the GUK domain (Fig. 3B, below dotted line). The domain binding energies of the four models are listed in Table 2 .

Table 2. Binding energies (in $\mathrm{kcal} / \mathrm{mol}$ ) of four SH3/GuK module combinations, predicted from homology models.

\begin{tabular}{|c|c|c|}
\hline GH3-domain & MPP4 & MPP5 \\
\hline MPP4 & 147 & 118 \\
\hline MPP5 & 144 & 137 \\
\hline
\end{tabular}

The binding energies of the SH3-GUK module of MPP4 and -5 are predicted according to the NOVA energy function (in kilocalories per mole). Results were obtained by subtracting the NOVA energy of the SH3/GUK complex from the energies of the separated SH3 and GUK modules; higher energies thus indicate better binding. The highest binding energy (147 kcal.mol) is predicted for the SH3/GUK self-interaction in MPP4. This matches our experimental finding in the yeast two-hybrid system that MPP4 a full length MPP4 construct did not bind to MPP5 (data not shown), but obviously prefers the self-interaction. The second strongest binding energy was obtained for $\mathrm{MPP}^{\mathrm{SH}}{ }^{3}-\mathrm{MPP} 4^{\mathrm{GuK}}$. This model contains several salt bridges that are either missing or less pronounced 


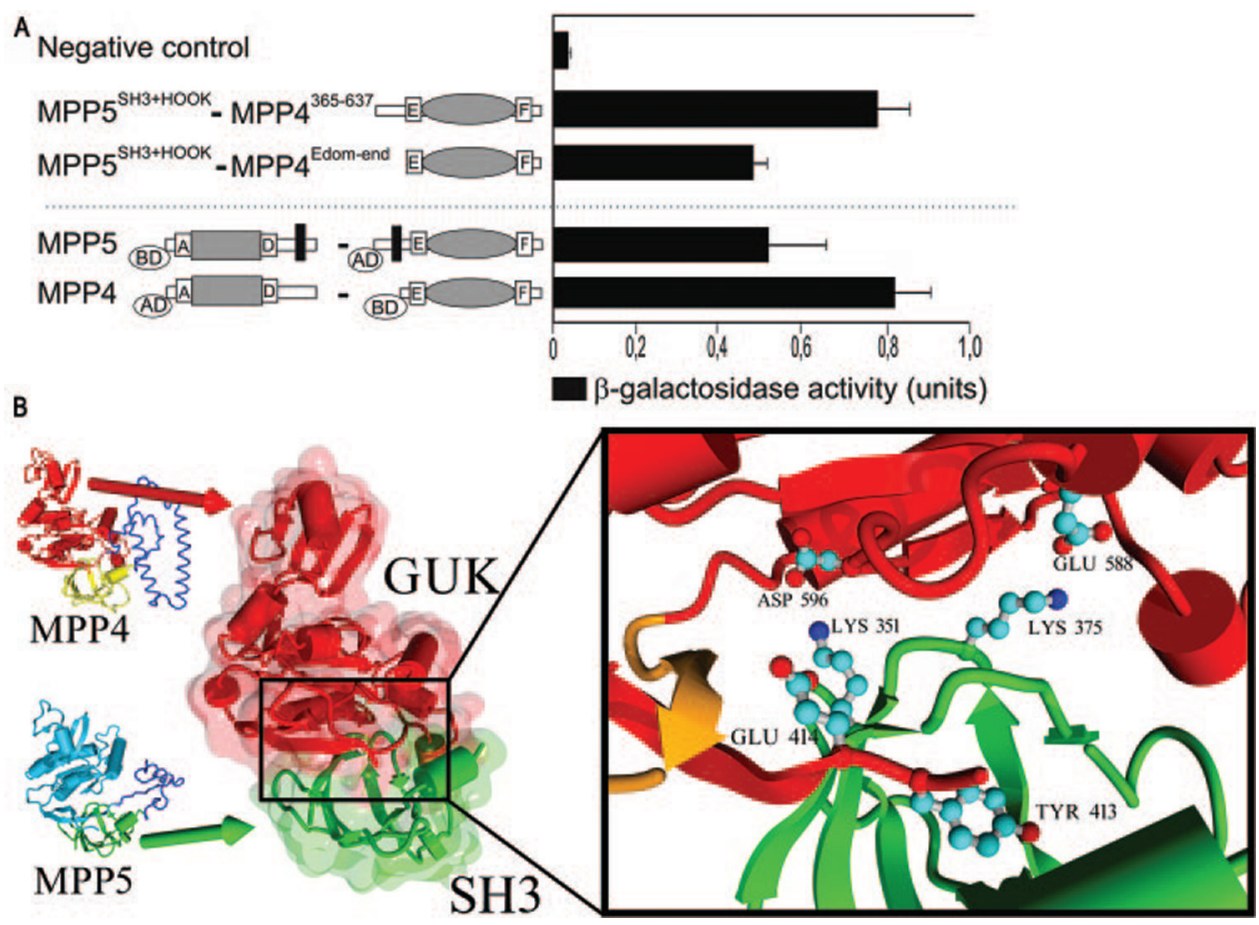

Fig. 3 Analysis of the interacting domains of MPP4 and -5.

(A). To quantify the intermolecular interaction between MPP4 and -5 (above dotted line), the YRG-2 yeast strain was cotransformed with the SH3-HOOK region of MPP5 together with two different deletion constructs of MPP4. The minimal region of MPP4 needed for interaction contains the E-GuK-F region. To determine the intramolecular interaction of MPP5 as well as MPP4 (below dotted line), yeast cells were co-transformed with two constructs of MPP5 (pBD-GAL4 domain fused to the SH3-HOOK region and the pADGAL4 domain fused to the HOOK-GuK domain), as well as two constructs of MPP4 (pBD-GAL4 domain fused to the GuK domain and pAD-GAL4 domain fused to the $\mathrm{SH} 3$ domain). Interactions were quantified by determining the activation of the LacZ reporter gene in a liquid ONPG assay ( $\beta$-galactosidase activity, black bars). As a negative control, the pBD-GAL4 domain fused to CRB1 $1^{\text {intra }}$ and the pAD-GAL4 domain fused to $\mathrm{MPP}^{\mathrm{PDZ}}$ was used. (B). Homology modeling of MPP4 and -5. The initial models are shown on the left, covering the $\mathrm{SH} 3$ (yellow, green) and GuK domains (red, light blue). By swapping the domains, one obtains a hetero-dimer, half of which is shown in the middle: the SH3 domain of MPP5 bound to the GuK domain of MPP4. Energy calculations predicted a high binding energy for this interaction (Table 2), due to a number of salt bridges, shown in the close-up: the triad Glu $414_{\mathrm{MPP} 4}$ - Lys $351_{\mathrm{MPP} 5}$ - Asp $596_{\mathrm{MPP} 4}$, then Lys $375_{\mathrm{MPP} 5}$ - Glu $588_{\mathrm{MPP} 4}$ and (not shown) Arg $418_{\text {MPP4 }}$ - Glu $395_{\text {MPP } 5}$. Note that an essential tyrosine (Y413) in the GUK domain of MPP4 sticks in the core of the SH3 domain of MPP5. 
in the remaining two models (Fig. 3B, right). Indeed, the MPP5 ${ }^{\mathrm{SH} 3}-\mathrm{MP}-$ $\mathrm{P} 4{ }^{\mathrm{GuK}}$ interaction was the one we first discovered experimentally in the yeast two-hybrid screening (Fig. 3A).

To further validate the model further, we analyzed the binding affinities of the SH3 and GUK domains in MPP4 and -5 (Fig. 3A, right). We were able to confirm in the sensitive liquid ONPG assay that the MPP4 domains interact more strongly than the ones in MPP5.

\section{Expression of CRB1, MPP5 and MPP4}

Expression analysis by semiquantitative RT-PCR on a panel of RNAs from several human tissues showed that $C R B 1$ is predominantly expressed in the retina. An increase in the number of PCR cycles identified a lower level of expression in brain, testis and fetal eye (Fig. 4). MPP5 is more ubiquitously expressed and is also present in the retina. MPP4 is highly expressed in the retina. An increase in the number of PCR cycles identified a lower level of expression in brain, testis, ARPE cell line and fetal eye. Low levels of MPP4 RNA were also detected in human retinal pigment epithelium.

Low levels of MPP4 RNA were also detected in human retinal pigment epithelium, but MPP4 protein was below detection levels in human and mouse RPE ${ }^{24}$.

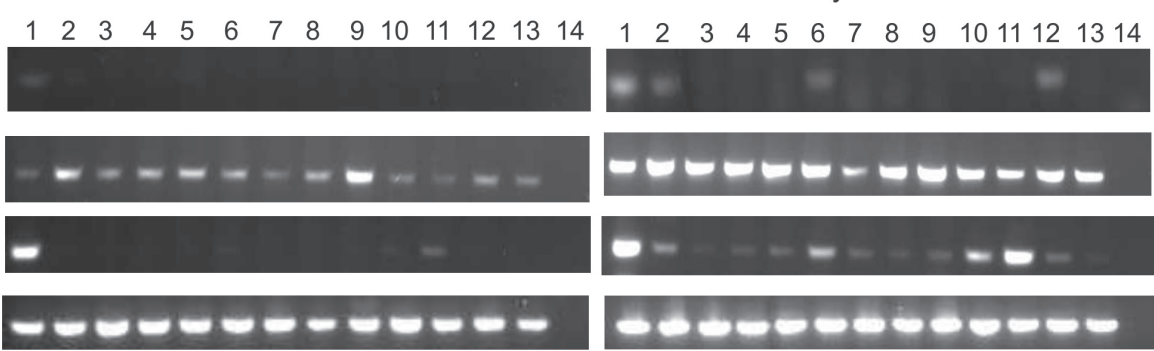

Fig. 4 mRNA expression profiles of CRB1, MPP5 and MPP4.

Expression profile of CRB1, MPP5 and MPP4 in human tissues and RPE cell line (ARPE) determined by RT-PCR.

1. retina; 2. brain; 3. skeletal muscle; 4. heart; 5. lung; 6. testis; 7. kidney; 8. liver;

9. placenta; 10. ARPE; 11. RPE; 12. fetal eye; 13. fetal cochlea; 14. negative water control. GAPDH served as a positive control. 


\section{Immunolocalizations of CRB1, MPP4 and MPP5 in human retina}

Immunohistochemistry and confocal laser scanning microscopy were used to determine the subcellular protein localization of CRB1, MPP4 and MPP5. Anti-CRB1 antibodies AK2 and -5 detected CRB1 at the outer limiting membrane (OLM) of human retina. Using monoclonal antibodies against human $\beta$-catenin as a marker for the adherens junctions, localization of CRB1 apical to the adherens junctions was detected (Fig. 5A-D). Anti-MPP5 antibodies (SN47) detected the protein at the OLM, also apical to the AJ (Fig. 5E-H), where it colocalized with CRB1 (Fig. 5I-L). MPP4 was detected at the OLM and in the outer plexiform layer (OPL) of human retina with AK4 (and AK8) antibodies (Fig. 5M-Q). At the OLM, MPP4 was detected apical to the adherens junction. However, the intensity of the signal at the OLM was much lower than the MPP4 staining intensity in the OPL (Fig. 5M-Q).

\section{Ultrastructural localization of mouse Mpp4 in retina}

Mpp4 was detected with immunoelectron microscopy at the OLM and in the cone pedicles and rod spherules. At the OLM, Mpp4 was present apically to and at the adherens junction contacts (Fig. 6A,E). Staining was also detected at membranes of the Golgi area (Fig. 6A asterisk, 6D) and other small and large vesicles in the inner segments of photoreceptors. In rod photoreceptors there was a strong association of the signal with the lateral plasma membranes of the spherules (Fig. 6B,F). In the cones, the Mpp4 signal was concentrated at the basal side, which comprises the contacts with horizontal and bipolar cells, and also at the lateral side of the pedicle plasma membrane (Fig. 6C asterisk, 6G). In both types of photoreceptors, Mpp4 staining was also associated with vesicles proximal to the pre-synaptic membrane.

The ultra-structural study showed that retinal Mpp4 is restricted to the photoreceptors. We did not detect any protein in the Múller (Fig. 6E), bipolar, horizontal or other neuronal retinal cell types. This finding correlates with in situ hybridization analysis on mouse retina showing distribution of mRNA coding for Mpp4 protein in the photoreceptor inner segments and outer nuclear layer (ONL) ${ }^{47,48}$. We conclude that Mpp4 is localized in rod and cone photoreceptors at the plasma membrane and at membranes of intracellular vesicles around the subapical region (SAR) and adherens junctions, and OPL. 

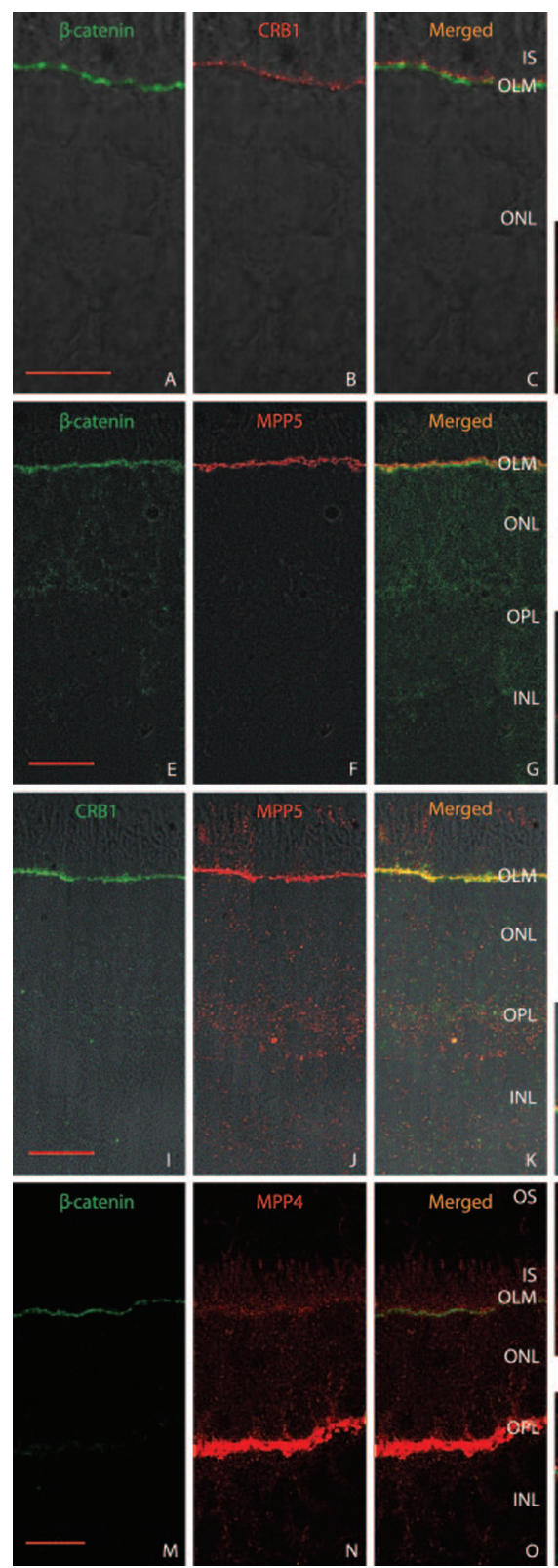

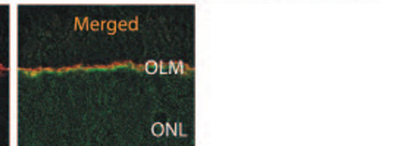

ONL

oph

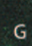

.

OLM detail

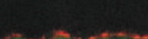

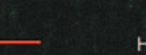

OPL

INL
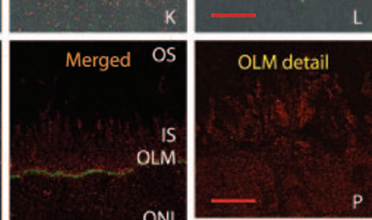

ONL

OP:

INL
OLM detail

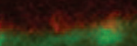

D

control secondary antibodies (P). Anti-ß-catenin antibody strongly stained the adherence junction (D,H,Q), whereas anti-CRB1 antibody AK2 (D,L), anti-MPP5 SN47 (H,L), and anti-MPP4 AK4 (N-O,Q) stained the SAR in the outer limiting membrane (OLM). MPP5 and CRB1 colocalize at the SAR (L). AK4 stained the OPL (N,O) and the OLM $(\mathrm{O}, \mathrm{Q})$, whereas secondary antibodies (P) produced some background staining in the photoreceptor inner and outer segments. IS, inner segments; ONL, outer nuclear layer; OPL, outer plexiform layer; OS, outer segments; INL, inner nuclear layer. Scale bars: (A-C, H, L, P, Q) $10 \mu \mathrm{m}$; (D, E-G, I-K, M-O) $20 \mu \mathrm{m}$. 


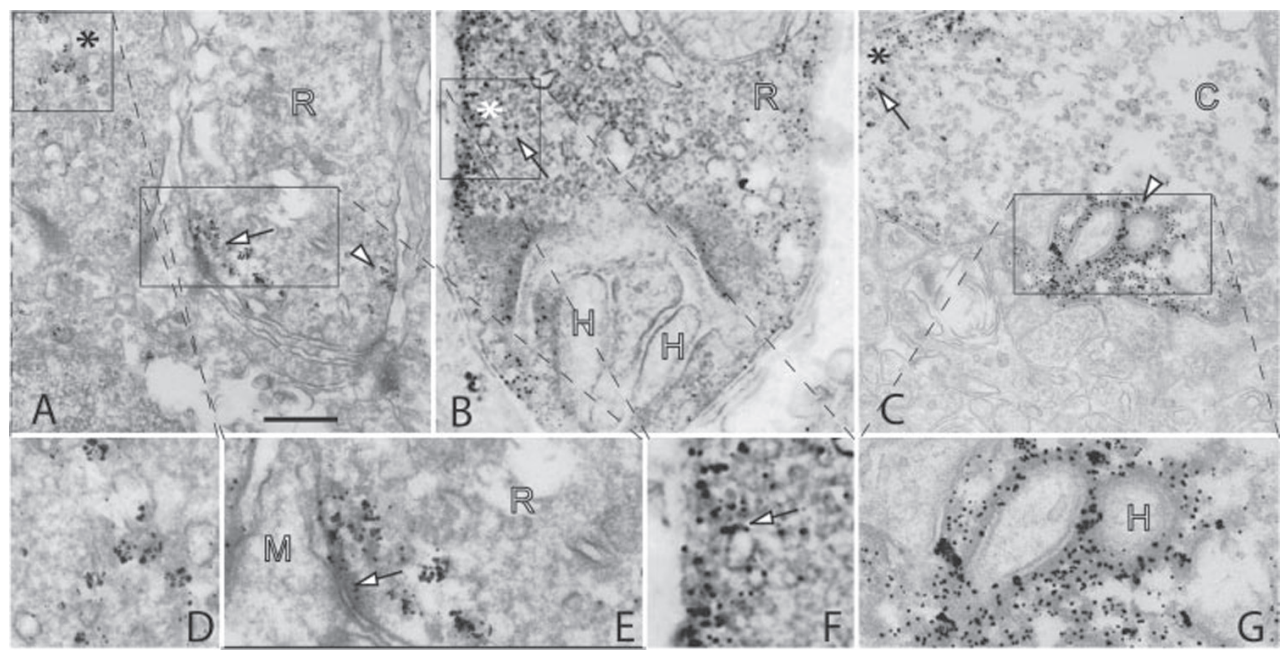

Fig. 6 Immuno-electron microscopy of mouse Mpp4 in retina.

(A). In the OLM, Mpp4 was located at the plasma membrane apical to and at the zonula adherens contacts (arrow head and arrows respectively). There is also staining at the trans Golgi network (asterisk). (B). In rod photoreceptors the lateral plasma membranes of the spherules were strongly stained (asterisk). Mpp4 staining was also found associated with vesicles proximal to the presynaptic membrane (arrow). (C). Mpp4 was found at the basal side of cone pedicles, where it is concentrated at the contacts with horizontal cell processes and bipolar cell dendrites (arrowhead), and also at the plasma membrane of the lateral side of the pedicle membrane (asterisk). As in rods, Mpp4 staining was also associated with vesicles proximal to the pre-synaptic membrane (arrow). (D). Detailed view of the staining of the Golgi area in the inner segment of a rod. (E). Magnification of the AJ showing the presence of the signal only at the photoreceptor side, but not in the Múller glia cell. (F). MPP4 is located at the pre-synaptic plasma membrane, and proximal vesicles (arrow). (G). At the cone synapse, the signal is associated with the pre-synaptic membrane.

Scale bar represents $0.5 \mu \mathrm{m}$. R-rod photoreceptor, C-cone photoreceptor, Hhorizontal cell, M- Múller glia cell.

\section{DISCUSSION}

\section{MPP4 as a member of the CRB1 protein scaffold}

Recent findings have emphasized the central role of CRB1 and its intracellular interactor MPP5/PALS1 in the regulation of epithelial polarity. However, the larger part of both CRB1, containing a putative FERMbinding motif, and MPP5, containing the additional MAGUK modules, have not yet been described to have a role in this process. The putative protein-protein interacting capacity of these domains in any tissue was 
still to be shown. This motivated us to search for interactors with these specific domains in the retina using a yeast two-hybrid approach. In this study, our results showed that the MAGUK protein MPP4 is recruited to the MPP5/CRB1 complex through direct binding of the SH3-GUK modules of both MPP family members. These three proteins form a multiprotein complex at the OLM of the retina. Using antibodies directed against the multiple PDZ protein Mupp1, we recently showed coimmunoprecipitation of endogenous Mpp4, Mpp5 and Crb1 from mouse retinal lysates of wild type mice, and coimmunoprecipitation of Mpp4 and -5 from lysates of $\mathrm{Crb1}$ knockout mice ${ }^{24}$. We now have identified MPP4 as a binding partner for the SH3-HOOK region of MPP5.

\section{Mechanism of interaction and implications for regulation}

The interaction between MPP5 and -4 involves the C-terminal end of MPP4. Alternative interaction through L27 domain dimerization was excluded. In a yeast two-hybrid experiment, no L27 domain binding was found between MPP4 and -5, whereas the L27 domains of PATJ and MPP5 did show interaction (data not shown).

Detailed analysis revealed that the $\mathrm{E}$ and $\mathrm{F}$ strands flanking the GUK domain of MPP4 are essential for binding. This is in full agreement with the folding according to the crystal structure of PSD-95 ${ }^{40,46}$. In PSD-95, the SH3-HOOK region interacts both in cis and in trans with the E-GUK-F region in vitro. The intramolecular interaction initially prohibits intermolecular interactions by preventing a mechanism called 3D domain swapping. Domain swapping allows proteins to assemble dimers or higher order oligomers by exchanging complementary substructures 49-51. The need of a cofactor or modulator has been postulated to switch the preference from intra- towards intermolecular interactions in vivo, thus enabling specific regulation of MAGUK multimerization at the cytoplasmic membrane ${ }^{40,46}$.

The 3D homology modeling of MPP5 and -4 pointed to a PSD95-like interaction mechanism that also matched the results of our yeast two-hybrid binding assays. GST pull-down analysis and immunoprecipitation experiments both confirmed the interactions biochemically and provided in vivo evidence for the proposed regulatory mechanism of heterodimerization. The full-length proteins that were the targets for immunoprecipitation did coprecipitate the full complex of CRB1, MPP4 and MPP5 from HEK293 cells, indicating that in these cells the regulatory factor is present (Fig. 2C,D). Good candidates for regulation of 
the dimerization of MPP5 are members of the 4.1 protein family, as the variable hinge or HOOK region of MPP5 contains a conserved 4.1 binding motif. MPP4 does not contain this particular conserved motif in the same region, but does have a predicted $\alpha$-helical stretch. We propose that regulation of dimerization of these MAGUK proteins is one of the factors that governs a dynamic variation of proteins that are present at this polarity-associated protein scaffold.

SH3 domains of tyrosine kinases are usually involved in proteinprotein interactions by binding to proline-rich sequences ${ }^{52,53}$, and have been described to couple substrates to enzymes, thereby regulating enzymatic activities ${ }^{54}$. However, based on the 3D homology models presented herein and the interactions identified in this study, the $\mathrm{SH} 3$ module of MPP4 and -5 seems to be functionally different from the conventional ones. The SH3 domain of MPP5 interacts with the GUK domain of MPP4 as well as with its own GUK domain.

\section{Distribution of protein expression in the human retina}

Whereas MPP5 is expressed ubiquitously ${ }^{7}$, both CRB1 and MPP4 genes are expressed more selectively in the eye and brain ${ }^{55}$. MPP4 RNA is also present in liver, spleen, heart ${ }^{47,48,55,56}$, and in testis but at much lower levels. Previous experiments showed that $C r b 1$ and $M p p 4$ RNA is expressed in the outer nuclear layer (ONL) and inner photoreceptor segments of the retina ${ }^{47,55}$. Crb1 RNA was also detected at low levels in the inner nuclear layer.

The protein complex CRB1-MPP5-MPP4 localizes subapically to the adherens junction at the OLM of the retina. The localization of Mpp4/MPP4, Mpp5/MPP5 and Crb1/CRB1 appears to be conserved between mice and humans ${ }^{24}$. Our results on MPP4 localization partially overlap with the positioning described for MPP4 in mouse retina ${ }^{48}$. Differences in genetic background or detection level may explain why Mpp4 was not detected in cones or OLM in previous studies ${ }^{48}$, and may explain the localization of MPP4 in the connecting cilia of bovine and porcine 57 .

We observed that Mpp4 is detected at intracellular vesicles and at the plasma membrane. Mpp4 is also located at the presynaptic membrane and proximal vesicles. The localization of MPP4 at more than one functionally different cell structure suggests participation in different protein complexes. Some MAGUK proteins target and anchor glutamate receptors to the synaptic terminals ${ }^{58}$. It has been proposed that the complex 
involving the MAGUK protein CASK acts as a nucleation site for the assembly of proteins involved in synaptic vesicle exocytosis and synaptic junctions ${ }^{59,60}$. It is tempting to speculate on possible functions of MPP4 in vesicle targeting or fusion complexes at the photoreceptor synapses and the region apical to the adherens junction.

\section{Implications for inherited retinal degenerations}

Altogether, these facts provide strong evidence for the involvement of CRB1, MPP5 and MPP4 in a common pathway that determines the polarity of photoreceptors in the retina. Based on the recruitment of both MPP4 and -5 to the CRB1 protein scaffold, the disruption of retinal lamination observed with loss of mouse Crb1 ${ }^{24}$ and the zebra fish MPP5 homologue Nagie oko ${ }^{14}$, and the high expression of MPP4 in the retina, we propose that MPP5 as well as MPP4 are functional candidate genes for inherited retinal degenerations.

The MPP4 gene has previously been screened for mutations in 300 $\mathrm{RP}$ patients, and it was mapped in a locus for autosomal recessive retinitis pigmentosa (RP26 locus) on 2q31-33, but no mutations were identified. Recently, mutations in the neighboring CERKL gene were found to cause RP26 ${ }^{61}$. However, based on the more severe phenotype that is often observed in patients with mutations in CRB1, MPP4 remains a candidate gene for similar eye disorders. Mutational changes in members of the complex that are closely linked could lead to a similar disrupting effect of the protein complex in the retina. Mutation analysis in selected patient panels could reveal the involvement of either MPP4 or MPP5 in inherited retina disorders.

\section{ACKNOWLEDGEMENTS}

The authors would like to thank Willem Kamphuis and Serge van de Pavert for advice and critical discussions on immunohistochemical data, and Anna Malysheva, Jan Meuleman, Paulus de Jong for comments on the manuscript; and Maarten L. Arends for excellent technical assistance. 


\section{REFERENCES}

1. Kowalczyk, A. P. \& Moses, K. Photoreceptor cells in flies and mammals: Crumby homology? Dev.Cell 2, 253254 (2002).

2. Altschuler, Y., Hodson, C., \& Milgram, S. L. The apical compartment: trafficking pathways, regulators and scaffolding proteins. Curr.Opin.Cell Biol. 15, 423-429 (2003).

3. Itoh, M. et al. Direct binding of three tight junction-associated MAGUKs, $\mathrm{ZO}-1, \mathrm{ZO}-2$, and ZO-3, with the $\mathrm{COOH}$ termini of claudins. J.Cell Biol. 147, 1351-1363 (1999).

4. Fanning, A. S., Jameson, B. J., Jesaitis, L. A., \& Anderson, J. M. The tight junction protein ZO-1 establishes a link between the transmembrane protein occludin and the actin cytoskeleton. J.Biol.Chem. 273, 2974529753 (1998).

5. Anderson, J. M. Cell signalling: MAGUK magic. Curr.Biol. 6, 382-384 (1996).

6. Woods, D. F. \& Bryant, P. J. The discs-large tumor suppressor gene of Drosophila encodes a guanylate kinase homolog localized at septate junctions. Cell 66, 451-464 (1991).

7. Kamberov, E. et al. Molecular cloning and characterization of Pals, proteins associated with mLin-7. J.Biol. Chem. 275, 11425-11431 (2000).

8. Gumbiner, B., Lowenkopf, T., \& Apatira, D. Identification of a 160$\mathrm{kDa}$ polypeptide that binds to the tight junction protein ZO-1. Proc.Natl. Acad.Sci.U.S. A 88, 3460-3464 (1991).

9. Gonzalez-Mariscal, L., Betanzos, A., \& Avila-Flores, A. MAGUK proteins: structure and role in the tight junction. Semin.Cell Dev.Biol. 11, 315324 (2000).
10. Roh, M. H. et al. The Maguk protein, Pals1, functions as an adapter, linking mammalian homologues of Crumbs and Discs Lost. J.Cell Biol. 157, 161-172 (2002).

11. Leonoudakis, D. et al. Protein trafficking and anchoring complexes revealed by proteomic analysis of inward rectifier potassium channel (Kir2.x)associated proteins. J.Biol.Chem. 279, 22331-22346 (2004).

12. Dimitratos, S. D., Woods, D. F., Stathakis, D. G., \& Bryant, P. J. Signaling pathways are focused at specialized regions of the plasma membrane by scaffolding proteins of the MAGUK family. Bioessays 21, 912-921 (1999).

13. Knust, E., Tepass, U., \& Wodarz, A. crumbs and stardust, two genes of Drosophila required for the development of epithelial cell polarity. Dev. Suppl 261-268 (1993).

14. Wei, X. \& Malicki, J. nagie oko, encoding a MAGUK-family protein, is essential for cellular patterning of the retina. Nat.Genet. 150-157 (2002).

15. Izaddoost, S., Nam, S. C., Bhat, M. A., Bellen, H. J., \& Choi, K. W. Drosophila Crumbs is a positional cue in photoreceptor adherens junctions and rhabdomeres. Nature 416, 178-183 (2002).

16. Pellikka, M. et al. Crumbs, the Drosophila homologue of human CRB1/ $\mathrm{RP} 12$, is essential for photoreceptor morphogenesis. Nature 416, 143-149 (2002).

17. Hong, Y., Ackerman, L., Jan, L. Y., \& Jan, Y. N. Distinct roles of Bazooka and Stardust in the specification of Drosophila photoreceptor membrane architecture. Proc.Natt.Acad.Sci.U.S.A 100, 12712-12717 (2003). 
18. Hong, Y., Stronach, B., Perrimon, N., Jan, L. Y., \& Jan, Y. N. Drosophila Stardust interacts with Crumbs to control polarity of epithelia but not neuroblasts. Nature 414, 634-638 (2001).

19. Grawe, F., Wodarz, A., Lee, B., Knust, E., \& Skaer, H. The Drosophila genes crumbs and stardust are involved in the biogenesis of adherens junctions. Development 122, 951-959 (1996).

20. Wodarz, A., Hinz, U., Engelbert, M., \& Knust, E. Expression of crumbs confers apical character on plasma membrane domains of ectodermal epithelia of Drosophila. Cell 82, 67-76 (1995).

21. Tepass, U., Theres, C., \& Knust, E. crumbs encodes an EGF-like protein expressed on apical membranes of Drosophila epithelial cells and required for organization of epithelia. Cell 61, 787-799 (1990).

22. Bachmann, A., Schneider, M., Theilenberg, E., Grawe, F., \& Knust, E. Drosophila Stardust is a partner of Crumbs in the control of epithelial cell polarity. Nature 414, 638-643 (2001).

23. Makarova, O., Roh, M. H., Liu, C. J., Laurinec, S., \& Margolis, B. Mammalian Crumbs 3 is a small transmembrane protein linked to protein associated with Lin-7 (Pals1). Gene 302, 21-29 (2003).

24. Van de Pavert, S. A. et al. Crumbs homologue 1 is required for maintenance of photoreceptor cell polarization and adhesion during light exposure. J.of Cell Sci. 117, 4169-4177 (2004).

25. den Hollander, A. I. et al. Mutations in a human homologue of Drosophila crumbs cause retinitis pigmentosa (RP12). Nat.Genet. 23, 217-221 (1999).

26. Lotery, A. J. et al. Mutations in the CRB1 gene cause Leber congenital amaurosis. Arch.Ophthalmol. 119, 415420 (2001).

27. den Hollander, A. I. et al. Leber congenital amaurosis and retinitis pigmentosa with Coats-like exudative vasculopathy are associated with mutations in the crumbs homologue 1 (CRB1) gene. Am.J.Hum.Genet. 69, 198-203 (2001).

28. Bernal, S. et al. Study of the involvement of the RGR, CRPB1, and CRB1 genes in the pathogenesis of autosomal recessive retinitis pigmentosa. J.Med.Genet. 40, e89 (2003).

29. Lotery, A. J. et al. CRB1 mutations may result in retinitis pigmentosa without para-arteriolar RPE preservation. Ophthalmic Genet. 22, 163-169 (2001).

30. Mehalow, A. K. et al. CRB1 is essential for external limiting membrane integrity and photoreceptor morphogenesis in the mammalian retina. Hum.Mol.Genet. 12, 2179-2189 (2003).

31. Meuleman, J., Van de Pavert, S. A., \& Wijnholds, J. Crumbs homologue 1 in polarity and blindness. Biochem.Soc. Trans. 32, 828-830 (2004).

32. Guan, K. L. \& Dixon, J. E. Eukaryotic proteins expressed in Escherichia coli: an improved thrombin cleavage and purification procedure of fusion proteins with glutathione Stransferase. Anal.Biochem. 192, 262-267 (1991).

33. Fromont-Racine, M., Rain, J. C., \& Legrain, P. Building protein-protein networks by two-hybrid mating strategy. Guide to Yeast Genetics and Molecular and Cell Biology, Pt B 350, 513-524 (2002).

34. Roepman, R., Schick, D., \& Ferreira, P. A. Isolation of retinal proteins that interact with retinitis pigmentosa GTPase regulator by interaction trap screen in yeast. Methods Enzymol. 316, 
688-704 (2000).

35. Frangioni, J. V. \& Neel, B. G. Solubilization and purification of enzymatically active glutathione S-transferase (pGEX) fusion proteins. Anal. Biochem. 210, 179-187 (1993).

36. Dunn, K. C., Aotakikeen, A. E., Putkey, F. R., \& Hjelmeland, L. M. Arpe-19, A Human Retinal-Pigment Epithelial-Cell Line with Differentiated Properties. Investigative Ophthalmology \& Visual Science 36, S766 (1995).

37. den Hollander, A. I. et al. Isolation and mapping of novel candidate genes for retinal disorders using suppression subtractive hybridization. Genomics 58, 240-249 (1999).

38. Klooster, J. \& Vrensen, G. F. The ultrastructure of the olivary pretectal nucleus in rats. A tracing and GABA immunohistochemical study. Exp. Brain Res. 114, 51-62 (1997).

39. Kelley, L. A., MacCallum, R. M., \& Sternberg, M. J. Enhanced genome annotation using structural profiles in the program 3D-PSSM. J.Mol.Biol. 299, 499-520 (2000).

40. McGee, A. W. et al. Structure of the SH3-guanylate kinase module from PSD-95 suggests a mechanism for regulated assembly of MAGUK scaffolding proteins. Mol.Cell 8, 12911301 (2001).

41. Rost, B. Twilight zone of protein sequence alignments. Protein Eng 12, 85-94 (1999).

42. Vriend, G. WHAT IF: a molecular modeling and drug design program. J.Mol.Graph. 8, 52-6, 29 (1990).

43. Chinea, G., Padron, G., Hooft, R. W., Sander, C., \& Vriend, G. The use of position-specific rotamers in model building by homology. Proteins $23,415-$ 421 (1995).

44. Krieger, E., Koraimann, G., \&
Vriend, G. Increasing the precision of comparative models with YASARA NOVA--a self-parameterizing force field. Proteins 47, 393-402 (2002).

45. Krieger, E. et al. A structural and dynamic model for the interaction of interleukin-8 and glycosaminoglycans: support from isothermal fluorescence titrations. Proteins 54, 768-775 (2004).

46. Tavares, G. A., Panepucci, E. H., \& Brunger, A. T. Structural characterization of the intramolecular interaction between the $\mathrm{SH} 3$ and guanylate kinase domains of PSD-95. Mol.Cell 8, 1313-1325 (2001).

47. Conte, I. et al. Characterization of MPP4, a gene highly expressed in photoreceptor cells, and mutation analysis in retinitis pigmentosa. Gene 297, 33 38 (2002).

48. Li, M., Zhang, S. S., \& Barnstable, C. J. Developmental and tissue expression patterns of mouse Mpp4 gene. Biochem.Biophys.Res.Commun. 307, 229-235 (2003).

49. Bennett, M. J., Schlunegger, M. P., \& Eisenberg, D. 3D domain swapping: a mechanism for oligomer assembly. Protein Sci. 4, 2455-2468 (1995).

50. Schlunegger, M. P., Bennett, M. J., \& Eisenberg, D. Oligomer formation by 3D domain swapping: a model for protein assembly and misassembly. Adv.Protein Chem. 50, 61-122 (1997).

51. Newcomer, M. E. Trading places. Nat.Struct.Biol. 8, 282-284 (2001).

52. Ren, R., Mayer, B. J., Cicchetti, P., \& Baltimore, D. Identification of a ten-amino acid proline-rich SH3 binding site. Science 259, 1157-1161 (1993).

53. Musacchio, A., Saraste, M., \& Wilmanns, M. High-resolution crystal structures of tyrosine kinase $\mathrm{SH} 3$ domains complexed with proline-rich peptides. Nat.Struct.Biol. 1, 546-551 
(1994).

54. Cohen, G. B., Ren, R., \& Baltimore, D. Modular binding domains in signal transduction proteins. Cell 80, 237-248 (1995).

55. den Hollander, A. I. et al. Isolation of Crb1, a mouse homologue of Drosophila crumbs, and analysis of its expression pattern in eye and brain. Mech.Dev. 110, 203-207 (2002).

56. Stohr, H. \& Weber, B. H. Cloning and characterization of the human retina-specific gene MPP4, a novel member of the p55 subfamily of MAGUK proteins. Genomics 74, 377-384 (2001).

57. Stohr, H., Stojic, J., \& Weber, B. H. Cellular localization of the MPP4 protein in the mammalian retina. Invest Ophthalmol.Vis.Sci. 44, 5067-5074 (2003).

58. Colledge, M. et al. Targeting of PKA to glutamate receptors through a MAGUK-AKAP complex. Neuron 27, 107-119 (2000).

59. Butz, S., Okamoto, M., \& Sudhof, T. C. A tripartite protein complex with the potential to couple synaptic vesicle exocytosis to cell adhesion in brain. Cell 94, 773-782 (1998).

60. Zhang, Y., Luan, Z., Liu, A., \& $\mathrm{Hu}, \mathrm{G}$. The scaffolding protein CASK mediates the interaction between rabphilin $3 a$ and beta-neurexins. FEBS Lett. 497, 99-102 (2001).

61. Tuson, M., Marfany, G., \& Gonzalez-Duarte, R. Mutation of CERKL, a novel human ceramide kinase gene, causes autosomal recessive retinitis pigmentosa (RP26). Am.J.Hum.Genet. 74, 128-138 (2004). 




\section{CHAPTER 3}

\section{MPP1 links the Usher protein network and the Crumbs protein complex in the retina}

Ilse Gosens ${ }^{1,4}$, Erwin van Wijk ${ }^{1,2,4}$, Ferry F. J. Kersten ${ }^{1,2,4}$, Elmar Krieger ${ }^{3,4}$, Bert van der Zwaag ${ }^{5}$, Tina Märker ${ }^{6}$, Stef J. F. Letteboer $^{1}$, Simone Dusseljee ${ }^{1}$, Theo Peters ${ }^{2}$, Henk A. Spierenburg ${ }^{5}$, Ingrid M. Punte ${ }^{1}$, Uwe Wolfrum ${ }^{6}$, Frans P. M. Cremers ${ }^{1,4}$, Hannie Kremer ${ }^{2 *}$, and Ronald Roepman ${ }^{1,4^{*}}$.

${ }^{*}$ Equal senior authors.

Departments of ${ }^{1}$ Human Genetics, and ${ }^{2}$ Otorbinolaryngology, ${ }^{3}$ Center for Molecular and Biomolecular Informatics, and ${ }^{4}$ Nijmegen Centre for Molecular Life Sciences, Radboud University Nijmegen Medical Centre, Geert Grooteplein Zuid 10, P.O. Box 9101, 6500 HB, Nijmegen, The Netherlands.

${ }^{5}$ Department of Pharmacology and Anatomy, Rudolf Magnus Institute of Neuroscience, University Medical Centre Utrecht, Utrecht, The Netherlands.

${ }^{6}$ Department of Cell and Matrix Biology, Institute of Zoology, Johannes Gutenberg University of Mainz, Mainz, Germany.

Published in Human Molecular Genetics (2007) Aug 15; 16 (16):2993-2003 



\section{ABSTRACT}

The highly ordered distribution of neurons is an essential feature of a functional mammalian retina. Disruptions in the apico-basal polarity complexes at the outer limiting membrane (OLM) of the retina are associated with retinal patterning defects in vertebrates. We have analyzed the binding repertoire of MPP5/Pals1, a key member of the apico-basal Crumbs polarity complex, that has functionally conserved counterparts in zebrafish (nagie oko) and Drosophila (Stardust). We show that MPP5 interacts with its MAGUK family member MPP1/p55 at the OLM. Mechanistically, this interaction involves heterodimerization of both MAGUK modules in a directional fashion. MPP1 expression in the retina throughout development resembles the expression of whirlin, a multi-PDZ scaffold protein and an important organizer in the Usher protein network. We demonstrate that both proteins interact strongly by both a classical PDZ domain-to-PDZ binding motif (PBM) mechanism, and a mechanism involving internal epitopes. MPP1 and whirlin colocalize in the retina at the OLM, at the outer synaptic layer, and at the basal bodies and the ciliary axoneme. In view of the known roles of the Crumbs and Usher protein networks, our findings suggest a novel link of the core developmental processes of actin polymerization and establishment/ maintenance of apico-basal cell polarity through MPP1. These processes, essential in neural development and patterning of the retina, may be disrupted in eye disorders that are associated with defects in these protein networks. 


\section{INTRODUCTION}

The specialized layered structure of the neural retina is a fundamental, conserved feature that is essential for the correct perception of detailed visual images. In addition to the layered assembly of the different retinal cell types and their processes, cells are also distributed in a highly organized fashion within the plane of each cell layer. This retinal organization allows the different cell types and their processes to form a dense meshwork of cell-to-cell contacts, necessary to transfer, process, and adapt to intercellular signaling information. Aside from the fact that the differentiated layers of the retina consist of seven major cell classes, studies in zebrafish have shown that all retinal cells originate from a single sheet of morphologically uniform neuroepithelium ${ }^{1}$. In fully stratified adult retinae, the apical cell junctions of this epithelium are retained, forming a layer of cell-cell adhesive contacts between photoreceptors and Müller glia cells: the outer limiting membrane (OLM). During development, retinal cell types migrate away from the neuroepithelium to form the inner retinal layers. Although little is known about the molecular cues guiding the positioning of neurons in the mammalian retina, the disturbed retinal patterning of the zebrafish nok ${ }^{2}$, ome ${ }^{3,4}$, bas $^{3}$, glo $^{3,5}$ and moe ${ }^{6}$ mutants indicates that the associated proteins, MPP5/Pals1 ${ }^{2,7}, \mathrm{Crb} 2 \mathrm{a}^{8}$, aPKC ${ }^{9}$, N-cadherin ${ }^{10}$ and EPB41L5/YMO1 respectively ${ }^{11,12}$, are crucial for retinal integrity. These proteins are all part of the apico-basal polarity complexes at tight junctions and adherens junctions of mammalian epithelial cells. In Drosophila, the asymmetric distribution of transmembrane protein Crumbs and its binding partner Stardust (Drosophila homologue of MPP5/ nok), is instrumental for the generation and maintenance of cell polarity and for the regulation of epithelial junction assembly ${ }^{13-15}$.

Mutations in human Crumbs homologue 1 (CRB1) lead to inherited retinal degenerations such as Leber congenital amaurosis (LCA) and retinitis pigmentosa $(\mathrm{RP})^{16}$. The encoded polypeptide localizes apically to the OLM of the mammalian retina ${ }^{17}$, where it interacts with MPP5 ${ }^{18,19}$. At this location MPP5 organizes a protein scaffold that includes the MAGUK family members MPP3 ${ }^{20}$ and MPP4 ${ }^{18}$. In addition, MPP5 has also been found to interact with Lin-7 ${ }^{21}$, PAR6 ${ }^{22}$, PATJ ${ }^{23}$, MUPP1 ${ }^{19}$, Ezrin ${ }^{24}$, and the neuronal GABA transporter GAT1 ${ }^{25}$.

We here describe the identification of the specific interaction between MPP5 and MPP1, a.k.a. erythrocyte protein p55, and their colocalization at the OLM. In addition to Crumbs protein complex members, some Usher proteins, including whirlin/CIP98 have been identified at 
the OLM ${ }^{26}$. Our results indicate that the multi-PDZ protein whirlin also binds to MPP1 at this subcellular site. In addition, whirlin-MPP1 colocalization was determined in the outer plexiform layer, containing the synaptic processes of the photoreceptors, and at the region of the photoreceptor cilium. Mutations in whirlin are implicated in isolated deafness (type DFNB31) ${ }^{27}$ and in Usher syndrome (type USH2D) ${ }^{28}$. In the inner ear, whirlin was found to be essential in stereocilia organization, and an important mediator of actin polymerization ${ }^{27,29}$. Our current findings suggest a link of the core developmental processes of actin polymerization and generation or maintenance of cell polarity through MPP1 interaction with MPP5 and whirlin.

\section{MATERIALS AND METHODS}

\section{Animals}

Wistar rats (Harlan, The Netherlands) and B6/129 F1 mice used for this study were housed under normal conditions with access to food and water ad libitum. All animals were treated in accordance with international and institutional guidelines.

\section{DNA constructs}

Human retinal cDNA was used to clone full length MPP5 as well as the SH3+HOOK domain (a.a. 337-477), HOOK-end (a.a. 408-675) as described previously ${ }^{18}$. cDNAs encoding human full-length whirlin (amino acids 1-907), PDZ1 (amino acids 138 -233) and PDZ2 (amino acids 279360) were cloned in the pDONR201 vector as previously described ${ }^{30}$. Full length human MPP1 was cloned using IMAGE clone \#2820598. All MPP1 and whirlin constructs were made by PCR using the GATEWAY cloning system (Invitrogen, Groningen, the Netherlands) using the full length constructs as a template according to the manufacturer's procedures. Gene-specific primers that were used are listed in table 2 in the supplemental materials and methods.

\section{Yeast two-hybrid}

A GAL4-based yeast two-hybrid system (Hybrizap, Stratagene) was used to screen for proteins that interact with MPP5. The DNA binding domain (pBD) fused to the SH3 and HOOK domain of MPP5 in PJ69-4A was used as bait on a human oligo-dT primed retinal cDNA library ${ }^{18}$. The human oligo-dT primed retinal cDNA library was transformed in PJ69- 
$4 \alpha$ and contained $2.1 \times 10^{6}$ primary clones. Positive clones were obtained by cell-to-cell mating with an efficiency of 3.4\%, resulting in a total number of $13 \times 10^{6}$ clones on selection plates lacking tryptophan, leucine, histidine and adenine. In total, 11 clones that contained MPP1 were selected based on growth on these plates and by $\alpha$-and $\beta$-galactosidase activity. To map the interacting domains of MPP5, MPP1 and whirlin, constructs fused to pAD and pBD were co-transformed in PJ694 $\alpha$. If yeast clones grow on selection plates and show coloring in the $\alpha$-and $\beta$-galactosidase activity assays, a protein pair is indicated positive for interaction.

\section{Antibodies}

For immunostaining the following antibodies were used: anti- $\beta$-catenin (1:500, Transduction laboratories), anti-MPP5 (SN47, 1:250, Dr. J. Wijnholds) ${ }^{18}$, anti-MPP1 mouse serum A01 (1: 300, Abnova), anti-MPP1 rabbit serum (1:300, Dr. A.H. Chishti) ${ }^{31}$, anti-whirlin raised against a GSTfusion protein encoding a fragment (a.a. 701-765) of the long isoform $(1: 500)^{26}$. Secondary antibodies were conjugated with Alexa 488 or Alexa 568 (Molecular probes, Leiden, the Netherlands). For immunoprecipitation the following antibodies were used: anti-MPP5 SN47, anti-MPP1 N-19 (Santa-Cruz), anti-whirlin ${ }^{26}$, mouse anti-chicken IgG clone CG-106 (Sigma). Secondary antibodies: goat anti-chicken HRP, donkey anti-goat HRP, rabbit anti-guinea pig HRP from Abcam were used.

\section{GST-pull down and immunoprecipitations}

IPTG inducible BL21-DE3 cells were transformed with GST$\mathrm{MPP}^{\mathrm{SH} 3+\mathrm{HOOK}} / \mathrm{pDest} 15$, GST-MPP1 1 -end $/ \mathrm{pDest} 15$, GST-MPP1 1 FL $/ \mathrm{pD}$ est15, GST-MPP1 ${ }^{\text {Cdel9 }} / \mathrm{pDest15,}$ GST-MPP1 ${ }^{\mathrm{GUK}} / \mathrm{pDest15,}$ His-MBP$\mathrm{MPP}^{\mathrm{SH} 3+\mathrm{HOOK}} / \mathrm{pDest} 566$ or His-MBP-MPP1 ${ }^{\mathrm{E}-\text { end }} / \mathrm{pDest} 566$. Bacterial cell lysates were prepared as previously described ${ }^{32}$. Equal amounts of blocked $(1.5 \mathrm{mg} / \mathrm{ml} \mathrm{BSA}$ ) glutathione Sepharose 4B beads (Amersham Pharmacia) with GST, GST fusion proteins or beads alone were incubated with $1 \mathrm{ml}$ of bacterial lysates containing His-MBP-fusion proteins overnight at $4^{\circ} \mathrm{C}$. HA-tagged full length whirlin or PDZ3 of whirlin was expressed in Cos-1 cells as described previously ${ }^{26}$. Cos-1 were lysed in $50 \mathrm{mM}$ Tris $\mathrm{pH} 7.5,150 \mathrm{mM} \mathrm{NaCl}, 0.5 \%$ Triton-X100 and passed several times through a needle. Equal amounts of Cos-1 cell lysate were incubated with GST-MPP1 fusion proteins overnight at $4^{\circ} \mathrm{C}$. After several washes with lysis buffer and TBS containing 1\% triton X-100 and 2 mM DTT, beads were boiled and proteins were resolved on SDS-PAGE. 
For Western blotting, proteins were electrophoretically transferred onto nitrocellulose membranes, blocked in 5\% milk (BioRad) and incubated with primary rabbit antibody anti-His (H-15, Santa Cruz) or mouse monoclonal anti-HA (Sigma) and secondary antibody goat anti-rabbit Alexa680 (Molecular probes) or goat anti-mouse IRDye800 (Rockland). The bands were visualized using the Odyssey infrared imaging system (LI-COR Biosciences).

For immunoprecipitations, bovine retinas obtained from the slaughterhouse or mouse retinas (P90) were used. Cytosolic and membrane fractions were prepared as described previously ${ }^{19}$. Mouse monoclonal anti-chicken IgGs were pre-coupled to Dynabeads protein G (Invitrogen, Groningen, the Netherlands, $15 \mu \mathrm{g} /$ reaction), followed by a second round of coupling of chicken anti-MPP5 antibody SN47 (10 $\mu \mathrm{g} /$ reaction). Both antibodies were covalently crosslinked to the magnetic beads using DMP (Pierce) according to manufacturer's procedures described in the Dynabeads protocol. These beads were incubated with the bovine retinal membrane fraction for 2 hours at $4^{\circ} \mathrm{C}$. MPP1 N-19 antibody $(10 \mu \mathrm{g} /$ reaction) was covalently coupled to Dynabeads protein G beads and subsequently incubated with the membrane fraction of mouse retinas for 2 hours at $4^{\circ} \mathrm{C}$. Protein $\mathrm{A} / \mathrm{G}$ agarose beads (Santa Cruz) were incubated with $2 \mu \mathrm{l}$ of whirlin antibody, followed by overnight incubation with precleared mouse retinal cytosolic extract. After incubations, the beads were pelleted and washed three times with extraction buffer or lysis buffer for the cytosolic fraction and membrane fraction respectively. Beads were boiled and proteins were resolved on SDS-PAGE. For Western blotting, proteins were electrophoretically transferred onto nitrocellulose or PVDF membranes, blocked with 5\% non-fat dry milk (Biorad) in PBST (0.1\% Tween) and analyzed with the appropriate primary and HRP secondary antibodies in 1\% milk in PBST. For the analysis of MPP1 in the coimmunoprecipitation experiment with MPP5, anti-MPP1 mouse serum A01 (Abnova) was used. Bands were visualized using Supersignal West Pico Chemiluminescent substrate from Pierce.

\section{Expression profiling}

Total RNA was isolated from different human tissues, a D407 cell line and an ARPE-19 cell-line as described previously ${ }^{33}$. For the semi quantitative RT-PCR, 3.1 $\mu \mathrm{g}$ RNA was reverse transcribed using random hexanucleotides ${ }^{34}$. A touchdown PCR was performed on $62 \mathrm{ng} \mathrm{cDNA}$ for the MPP1-7 genes and the housekeeping gene GUS, which served as a stan- 
dard. Used primer combinations are described in supplemental materials and methods.

\section{In situ hybridization}

Mouse embryos (E12.5-E18.5) and adult mice (P7 and P90) were collected and prepared for in situ hybridization as described previously ${ }^{26}$. The MPP1 probe contains the last 3 exons and part of the 3'UTR.

\section{Immunohistochemistry}

Unfixed eyes of Wistar rats (P20) and mice (P90) were isolated and frozen in melting isopentane. Cryosections of $10 \mu \mathrm{m}$ were made and treated with $0.01 \%$ Tween-20 in PBS followed by a blocking step with blocking solution $(0.1 \%$ ovalbumin, $0.5 \%$ fish gelatin in PBS) as described previously ${ }^{26}$. Sections were incubated overnight with primary antibody diluted in blocking solution and for 1 hour with secondary antibody conjugated to Alexa 488 or 568 (Molecular probes, the Netherlands). Sections were embedded with Prolong Gold Anti-fade (Molecular Probes). For imaging, a Zeiss Axioscop 2 fluorescence microscope with Axiovision software was used.

\section{Pre-embedding immunoelectron microscopy}

Labeling was performed as described previously ${ }^{35}$. Vibratome sections through mouse retina were stained by primary antibodies against whirlin and MPP1 and visualized by appropriate secondary antibodies (Vectastain ABC-Kit, Vector, England). After fixation with $0.5 \% \mathrm{OsO}_{4}$ specimen were embedded in araldite and ultrathin sections were analyzed with a FEI Tecnai 12 TEM.

\section{Molecular modeling of MPP1 and MPP5}

The homology model of MPP1 (Swiss Prot entry EM55_HUMAN) was built using the protocol described for MPP4 and MPP5 previously ${ }^{18}$. The modeling template was again the SH3-GUK module of Postsynaptic Density Protein 95 (PSD-95) with $\sim 40 \%$ sequence identity, solved at $1.8 \AA$ resolution ${ }^{36}$ (PDB ID $\left.1 \mathrm{KJW}\right)$. To estimate the relative affinities (Table 1) of the three homodimers (MPP1-1, MPP4-4, MPP5-5) and the three heterodimers (MPP1-4, MPP1-5, MPP4-5), molecular models of all nine different SH3-GUK complexes were derived by permutating the domains of the initial models and re-optimizing the side-chains with the molecular modeling program YASARA (www.yasara.org), such that the 
NOVA force field energy was minimal and binding energies could be calculated $18,37,38$. Coordinate files of the models are available from the authors upon request.

\section{RESULTS}

\section{MPP5 specifically interacts with MPP1}

Since MPP5 contains a number of protein-protein interaction domains (Fig. 1A), a scaffolding function was assigned to this protein. To identify interactors for the SH3+HOOK domain of MPP5, we previously screened a human oligo-dT primed retinal cDNA library and isolated MPP4 as an interacting protein ${ }^{18}$. As homology modeling suggested a potential heterodimerization of different MPP family members, we expanded the yeast two-hybrid screening using an optimized cell-to-cell mating protocol. Besides MPP4, we identified 13 prey clones expressing MPP1 (Fig. 1B), but no other clones for MPP family members. All MPP1 clones contained part of the SH3 domain, the HOOK domain and GUK domain. Unlike other MAGUK family members, MPP1 does not contain L27 domains. The MPP1 ${ }^{\mathrm{E}-\mathrm{end}}$ and $\mathrm{MPP} 1^{\mathrm{SH} 3+\mathrm{HOOK}}$ constructs were used in yeast two-hybrid (Fig. 1C) as well as biochemical assays (Fig. 1D and supplemental figure 7) to pinpoint the interacting domains of MPP1 and MPP5 and determine their binding specificity. Yeast two-hybrid analysis of several MPP1 and MPP5 constructs showed that the interaction is directional (Fig. 1C); the MPP1 ${ }^{\text {prey }}$ containing the GUK domain interacts with the $\mathrm{SH} 3+\mathrm{HOOK}$ domain of MPP5, but the $\mathrm{SH} 3+\mathrm{HOOK}$ domain of MPP1 lacks binding affinity for the GUK domain in MPP5 ${ }^{\mathrm{HOOK}-\text { end }}$. In addition, the SH3+HOOK domain of MPP1 can bind the MPP1 ${ }^{\text {E-end }}$ protein that contains the GUK domain. This indicates that, similar to other MPP and MAGUK proteins, the SH3 and GUK molecules are involved in intramolecular binding in the same fashion as its intermolecular binding with MPP5. This intramolecular binding may prevent homodimerization in vitro, as we could not detect interaction of the full length MPP1 proteins in the yeast two-hybrid assay. GST-pull down analysis confirmed the interaction between the SH3+HOOK domain of MPP5 and the Cterminus of MPP1 (Fig. 1D and supplemental figure 7). 
A

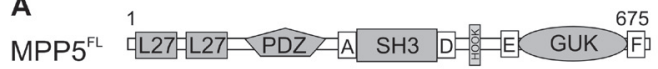
MPP $5^{\mathrm{SH} 3+\text { HоOK }}$ MPP5 ${ }^{\text {Hook-end }}$

\begin{abstract}
337
\end{abstract}$$
\underline{408}
$$
477

675

\section{C}

\begin{tabular}{|c|c|c|}
\hline pAD & pBD & Interaction \\
\hline MPP1 $^{\text {prey }}$ & $\mathrm{MPP}^{\mathrm{SH} 3+\mathrm{HOOK}}$ & + \\
\hline MPP1 ${ }^{\text {今Нзнноок }}$ & MPP5 $5^{\text {HOoK-end }}$ & - \\
\hline MPP5 $5^{\text {HOOK-end }}$ & MPP1 $1^{\text {Sн3+ноок }}$ & - \\
\hline$\overline{M P P} 1^{F L}$ & MPP1 $1^{\text {FL }}$ & - \\
\hline MPP1 $1^{\text {今нз+ноок }}$ & MPP1 $1^{\text {E-end }}$ & + \\
\hline MPP1 $1^{\text {E-end }}$ & MPP1 $1^{\mathrm{SH} 3+\mathrm{HOOK}}$ & + \\
\hline
\end{tabular}

B

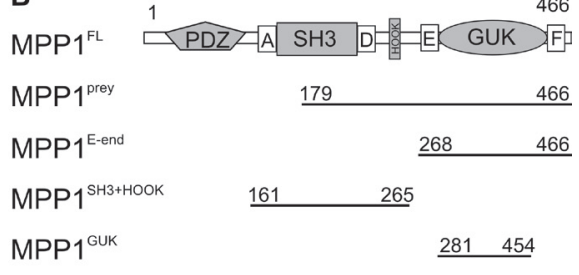

D

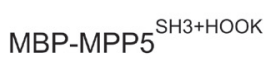

Control: MBP

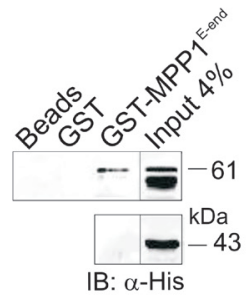

E

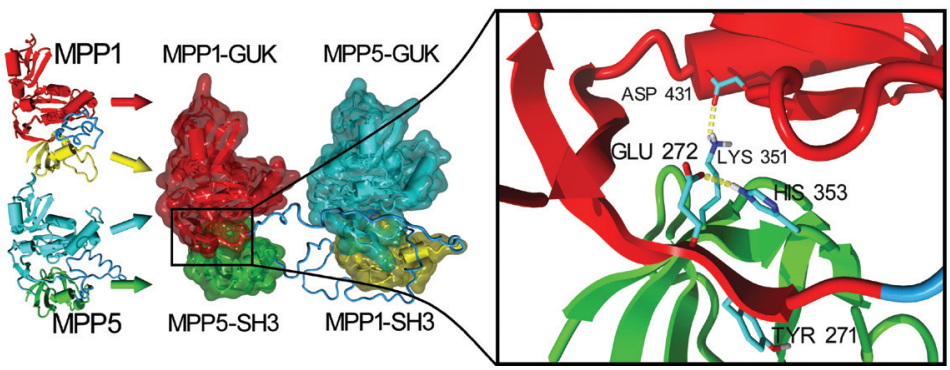

Fig. 1 MPP5 binds to MPP1 by a GUK/SH3 domain swap.

(A). Schematic representation of the overall domain composition of MAGUK protein MPP5. Two L27 domains and the MAGUK module containing a PDZ, SH3 and GUK domain are present. A HOOK domain is found between the SH3 and GUK domain. Strands A and D flanking the SH3 domain and E and $F$ flanking the GUK domain were identified according to homology with PSD-95. MPP5 ${ }^{\mathrm{SH} 3+\mathrm{HOOK}}$ was used as bait. The MPP5 ${ }^{\mathrm{HOOK}-\mathrm{end}}$ construct is used in a yeast two-hybrid assay to determine the specificity of the interaction between MPP1 and MPP5. (B). Identical MPP1 preys were found and MPP1 ${ }^{\mathrm{E}-}$ end, $\mathrm{MPP} 1^{\mathrm{SH} 3+\mathrm{HOOK}}, \mathrm{MPP}^{\mathrm{GUK}}$ and $\mathrm{MPP}^{\mathrm{FL}}$ constructs were made. $(C)$. The MPP1 ${ }^{\text {prey }}$ interacts with the $\mathrm{MPP}^{\mathrm{SH} 3+\mathrm{HOOK}}$, but $\mathrm{MPP}^{\mathrm{SH} 3+\mathrm{HOOK}}$ does not bind to MPP5 ${ }^{\text {HOOK-end. }}$. Full-length MPP1 proteins do not bind to each other, whereas selected parts of the MAGUK modules do. $(D)$. GST-MPP1 ${ }^{\mathrm{E}-\mathrm{end}}$ pulled down $\mathrm{MPP}^{\mathrm{SH} 3+\mathrm{HOOK}}$. Beads alone or GST did not interact with MPP5, while MPP1 did not bind the MBP tag in the control experiment, showing that this interaction is specific. (E). Homology modeling of the MAGUK modules of MPP1 and MPP5. The interacting GUK and SH3 domains of MPP1 (red and yellow, respectively) and MPP5 (cyan and green) separate and form a heterodimer consisting of two mixed GUK/SH3 complexes. The domain linker regions in 
MPP1 and MPP5 (shown in blue) are both long enough to support this rearrangement. The proposed mechanism of the MPP1-GUK/MPP5-SH3 (red, green) interaction is shown as a close-up: Lys 351 and His 353 in MPP5-SH3 interact with Asp 431 and Glu 272 in MPP1-GUK via a salt-bridge and a hydrogen bond, respectively. In addition, the GUK domain places Tyr 271 in the hydrophobic core of the $\mathrm{SH} 3$ domain, a strong but unspecific interaction found

\section{Analysis of MPP1- MPP5 interaction by molecular modeling}

In our initial work on the MPP4-MPP5 interaction, molecular modeling predicted a high binding affinity due to several salt-bridges ${ }^{18}$. Here we repeated the analysis not only for MPP1-MPP5, but also for all six homoand heterodimers that can be formed by MPP1, MPP4 and MPP5. In silico binding energies were calculated as described previously ${ }^{18}$ and are listed in Table 1. The predicted binding energy of the MPP4 homodimer was the highest, followed by MPP4-MPP5, while dimers involving MPP1 are ranked last. Visual inspection shows that this is mainly due to a smaller contact interface with fewer salt-bridges. Nevertheless, homology modeling of the MAGUK modules supports MPP1-MPP5 heterodimer formation (Fig. 1E), and is predicted to reach an affinity close to MPP5-MPP5 (Table 1).

Table 1. Estimated in silico binding energies for the six different dimers of MPP1, -4 and -5 , sorted from highest to lowest affinity. Each binding energy is the sum of the two GUK-SH3 interactions.

\begin{tabular}{|l|c|}
\hline Complex & Energy (kcal/mol) \\
\hline MPP4-MPP4 & 378 \\
\hline MPP4-MPP5 & 323 \\
\hline MPP5-MPP5 & 294 \\
\hline MPP1-MPP5 & 283 \\
\hline MPP1-MPP4 & 266 \\
\hline MPP1-MPP1 & 250 \\
\hline
\end{tabular}




\section{MPP1 binds MPP5 at the outer limiting membrane}

Expression analysis by semi-quantitative RT-PCR on a panel of RNAs from several human tissues showed that MPP1 is ubiquitously expressed as other MPP family members, except MPP4, which is mainly found in the retina (Supplemental figure 8). We used RNA in situ hybridization on mouse cryosections to monitor the cellular levels of MPP1 transcripts, and detected a distinct signal in the eye from E14.5 onwards. Intense staining was present in the liver and primitive gut, whereas the umbilical vein, the ventricular layer of the CNS and upper/ lower jaw region showed lower signal intensity (Fig. 2A). At E14.5 (Fig. 2B) and E16.5, expression in the liver and stomach was maintained. In addition, expression was present in bonestructures (e.g. zygomatic bone, lower jawbone), cranial nerve ganglia (e.g. trigeminal (V) ganglion), and cochlea (Fig. 2D). When viewed at higher magnification, the expression of MPP1 in the eye could be ascribed to the neuroblastic layer (Fig. 2F), the same cell layer in which the whirlin transcript was found to be expressed ${ }^{26}$. At E16.5 and E18.5 a slightly higher intensity of staining in the neuroblastic layer was seen (Fig. 2G, H). At E16.5, strong staining in the upper part of the gut was maintained and with the onset of ossification, expression was seen in all bone structures of the body (e.g. femur, Fig. 2E). At P7 and P90, MPP1 expression in the eye was identified in the ganglion cell layer, the inner nuclear layer (INL) and photoreceptor cell layer (Fig. 2I, J).

The subcellular localization of MPP1 in the retina was analyzed using polyclonal antibodies against MPP1 in cryosections of the rat retina. We detected MPP1 expression in the outer plexiform layer (OPL), the outer limiting membrane (OLM), the inner segments (IS), around the nuclei of the outer nuclear layer (ONL) and at the connecting cilium (CC) region that separates the inner and the outer segments, with both anti-MPP1 rabbit and mouse sera. In addition, MPP1 was detected in the retinal pigment epithelium (RPE) (Fig. 3A1). No staining in the inner nuclear layer, inner plexiform layer and ganglion cell layer was found (data not shown), although the MPP1 transcript was detected in the latter. Costaining with anti- $\beta$-catenin, a marker for the OLM and OPL, confirmed the presence of MPP1 at these locations (Fig. 3A2 and A3 and C2). Costaining with connecting cilium marker acetylated tubulin revealed some overlap, but most of the MPP1 signal is detected just below the axoneme of the connecting cilium (Fig 3B). Costaining of retinal sections with antibodies against MPP1 and MPP5 confirmed their colocalization at the OLM in the rat (Fig. 3C) as well as in the mouse retina (data not shown). 

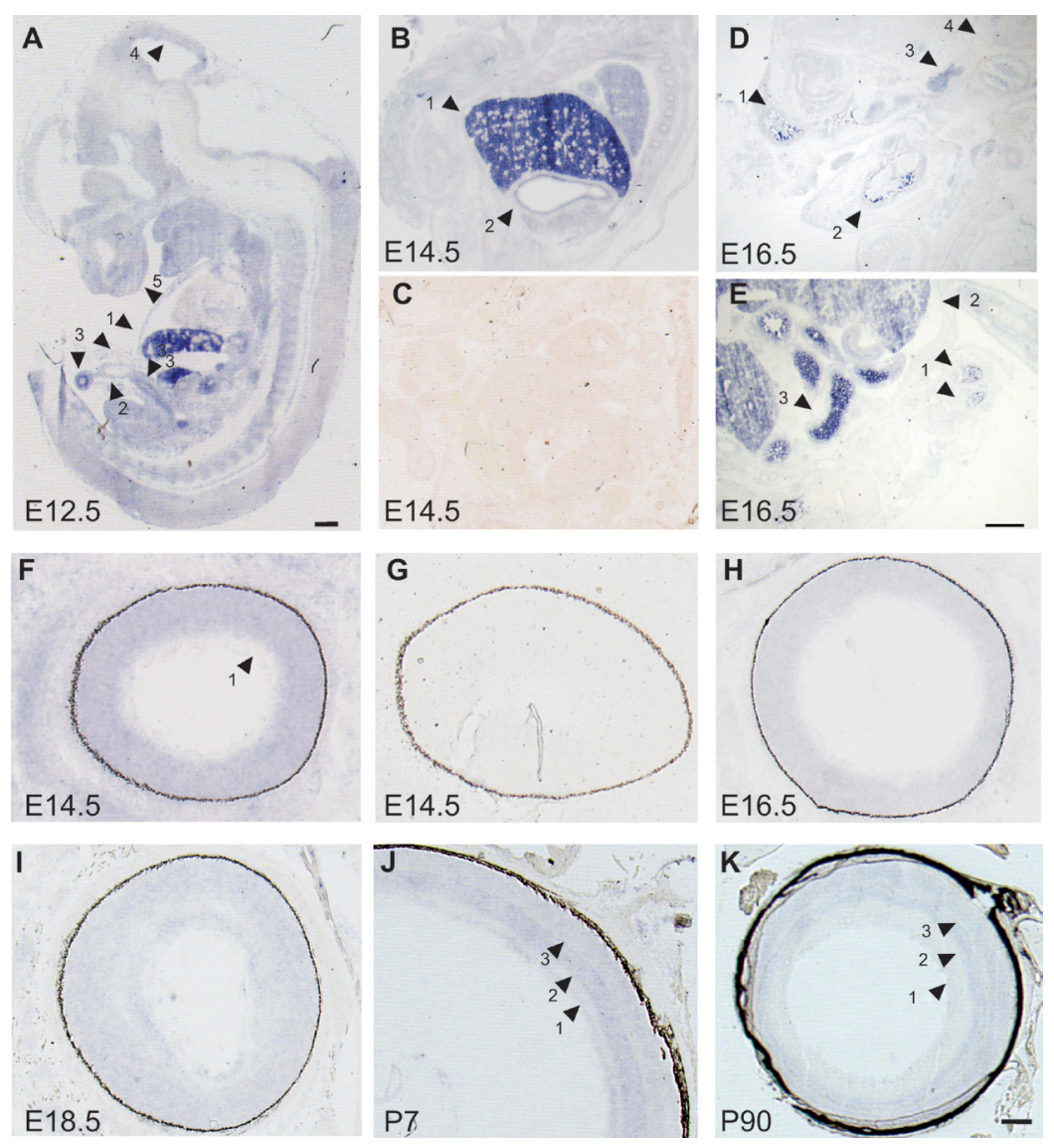

Fig. 2 MPP1 expression analysis by RNA in situ hybridisation on embryonic and adult mouse tissues.

In sagittal whole embryo cryosections low levels of expression were seen throughout the embryo. (A) MPP1 expression at E12.5 in 1. liver; 2. umbilical vein; 3. primitive gut; 4. neuroepithelium; and 5. upper jaw region. Scalebar is $300 \mu \mathrm{m}$. (B) At E14.5, expression was maintained in 1. the liver; and 2. stomach. (C) Hybridisation with a MPP1 sense probe did not show tissue labeling. (D) At E16.5, the 1. zygomatic bone; 2. lower jawbone; 3. trigeminal (V) ganglion; and 4. cochlea showed expression of MPP1. (E) A strong signal was also observed in 1. femur; 2. liver; and 3. small intestine at this stage. Scalebar is $20 \mu \mathrm{m}$. (F) In the eye, a slightly higher signal intensity compared to surrounding tissues was observed in the inner neuroblastic layer. $(G)$ No staining was observed in the eye after sense probe hybridisation. (H) At E16.5 and (I) E18.5 expression was maintained in the neuroblastic layer and appeared in the developing photoreceptor layer. (J) At P7 and (K) P90, expression in 1. ganglion cells; 2.inner nuclear layer (INL) and 3. photoreceptor cell layer was seen. Scalebar is $20 \mu \mathrm{m}$. 


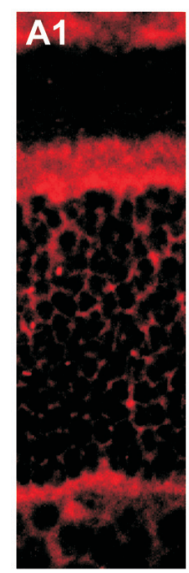

MPP1

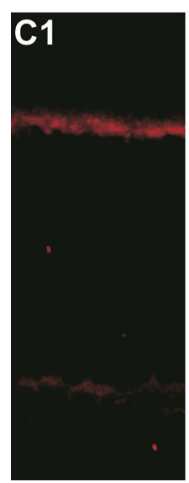

MPP5

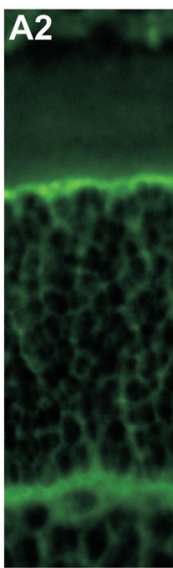

$\beta$-catenin

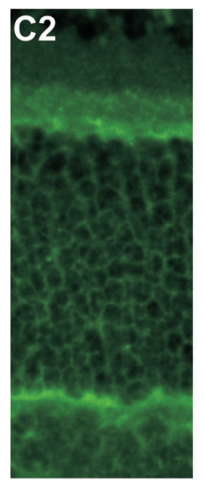

MPP1

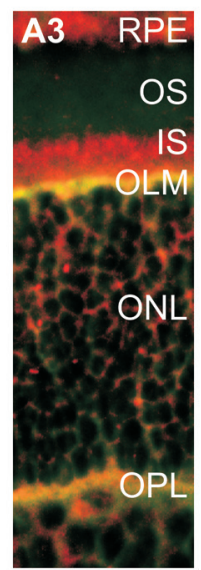

Merged

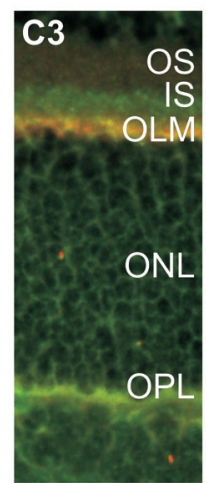

Merged

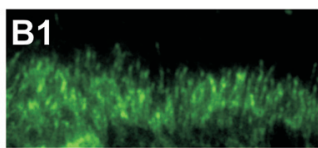

MPP1

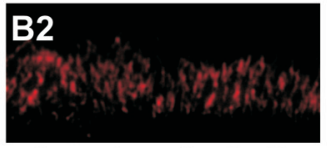

Acetylated tubulin

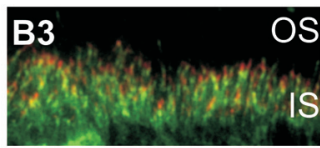

Merged

D

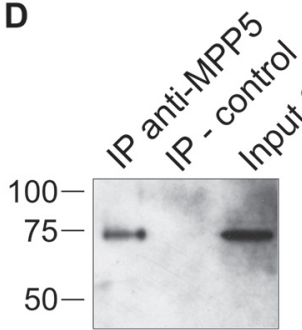

IB: $\alpha$-MPP5

IB: $\alpha-M P P 1$

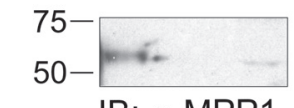

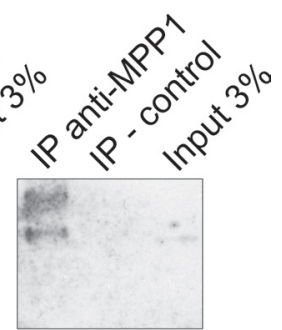

IB: $\alpha$-MPP5 
Immunoprecipitations from retinal lysates were performed to detect a physical interaction between MPP1 and MPP5. Anti-MPP5 antibody precipitated both MPP5 (75 kD) as well as MPP1 $(55 \mathrm{kD})$ from bovine and mouse retinas (Fig. 3D, left panels and data not shown). The reciprocal immunoprecipitation experiment using anti-MPP1, coprecipitated MPP5, confirming their presence in the same protein complex (Fig. 3D, right panel).

MPP1 binds whirlin at multiple subcellular locations in the retina Although a whirlin-MPP1 interaction has previously been described ${ }^{39}$, the mechanism of interaction was not analyzed in detail. We used different fragments of the long isoform of whirlin (Fig. 4A) to determine the epitopes interacting with the C-terminus of MPP1 that is homologous to the C-terminus of CASK (Fig. 4B), another known interactor of whirlin 39. Using yeast two-hybrid analysis, we could determine that PDZ3 of whirlin binds to the C-terminal region of MPP1 (MPP1 ${ }^{\mathrm{E}-\text { end }}$ ) containing the atypical PDZ binding motif (PBM). Constructs containing PDZ1+2 of whirlin did not bind (yeast two-hybrid data not shown). We could confirm binding of whirlin ${ }^{\text {PDZ3 }}$ to GST-MPP1 ${ }^{\mathrm{E}-\text {-end }}$, but not GST-MPP1 ${ }^{\text {GUK }}$ or unfused GST in a GST-pull down assay (Fig. 4C). Using homology modeling of the interaction of the PDZ binding motif at the $\mathrm{C}$-terminus of MPP1 with PDZ3 of whirlin, we were able to predict that this interaction is structurally feasible (Fig. 4D).

A

Whirlin long

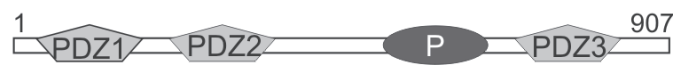

Whirlin short

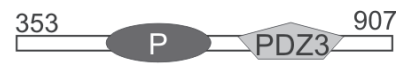

Whirlin $^{\text {PDZ3 }}$

561

907

Fig. 4 Whirlin can bind to MPP1 by two different mechanisms.

(A) Schematic representation of the long and short isoform of whirlin. The short isoform has an alternative starting sequence of 37 amino acids. Starting position is indicated relative to the long isoform. Both isoforms contain a proline rich region $(\mathrm{P})$ and the PDZ3 domain. A construct that contains the PDZ3 domain of whirlin is used for further biochemical analysis. 
B

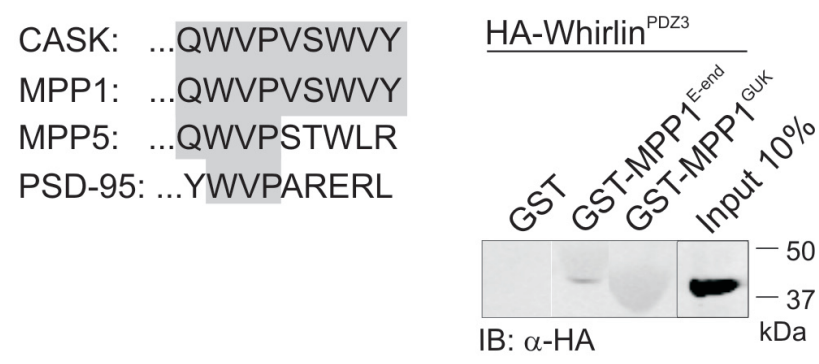

D

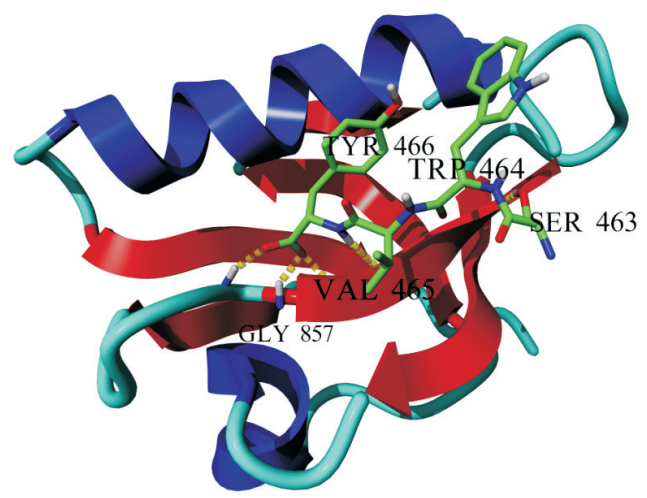

E

$\underline{\text { HA-Whirlin }^{\text {long }}}$

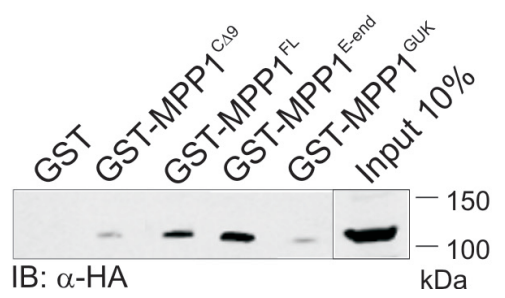

(B). Alignment of the nine C-terminal amino acids after the GUK domain of different MAGUK proteins. (C). Bacterially expressed GST-fusion proteins of MPP1 ${ }^{\mathrm{E}-\mathrm{end}}$ and $\mathrm{MPP} 1^{\mathrm{GUK}}$ were used to determine the minimal binding domain required for interaction with the PDZ3 domain of HA-tagged whirlin. GSTMPP1 ${ }^{\mathrm{E}-\mathrm{end}}$ did bind to whirlin ${ }^{\mathrm{PDZ}}$, whereas MPP1 containing only the GUK domain did not. Some background signal of GST-fusion proteins is detected in lane 2 and 3. (D). Homology modeling of the MPP1 C-terminus bound to the C-terminal whirlin PDZ3 domain (PDB 1UFX) indicates that the core motif of the interaction is the peptide's C-terminal carboxyl group tightly bound by three backbone NH groups, a common feature in PDZ-PBM interactions. However, this interaction is not possible in 1UFX (the whirlin PDZ structure) since one peptide plane is flipped and a $\mathrm{C}=\mathrm{O}$ faces the carboxyl group. Therefore, it is predicted that Gly 857 in whirlin must undergo a conformational 
change to allow binding, which is represented in the figure. $(E)$. The full length HA-tagged whirlin can bind to the GST-fused full-length MPP1 protein without the C-terminal 9 amino acid of MPP1 (GST-MPP1 ${ }^{\mathrm{C} \Delta 9}$ ). However, the interaction with $\mathrm{MPP}^{\mathrm{FL}}$, the full-length protein that contains the PDZ binding motif is stronger (equal amounts of proteins were used). The $\mathrm{C}$-terminal part of MPP1 (MPP1 ${ }^{\mathrm{E}-\mathrm{end}}$ ) also binds strongly to full length whirlin, whereas the GUK domain of MPP1 (MPP1 ${ }^{\mathrm{GUK}}$ ) without the PDZ binding motif still has reduced affinity.

Strikingly, when using a mutant MPP1 protein with a C-terminal 9 a.a. deletion in these interaction studies, binding to the long isoform of whirlin was not absent, only reduced compared to the full-length MPP1 protein. A similarly weak binding could be observed for full-length whirlin and the MPP1 ${ }^{\text {GUK }}$ fragment (Fig. 4E). These findings point to an additional interaction mechanism, besides the PDZ-PBM mechanism.

Recently, a complex of whirlin and MPP1 was described to localize to the stereocilia tips in hair cells of the inner ear ${ }^{29}$, but a similar complex in the retina has never been reported. Excitingly, the localization of MPP1 in the retina strongly resembled the localization pattern of whirlin ${ }^{26}$. Immunohistochemical analysis of rat retinal sections using antibodies against whirlin and MPP1 clearly confirmed the colocalization of both proteins at the OPL, the OLM, at the region of the connecting cilia, and somewhat weaker at the inner segments (Fig. 5A and B). In order to confirm the presence of both proteins in the same retinal protein complex, we performed co-immunoprecipitation analysis. The anti-whirlin antibody efficiently precipitated whirlin (Fig. 5C, top panel), and MPP1 coprecipitated as part of the same protein complex from the mouse retina (Fig. 5C, bottom panel). The distinct colocalization of MPP1 and whirlin at the connecting cilium is particularly interesting, as this region has been shown to harbor many proteins associated with inherited retinal degeneration. Mutations in the centrosome/ciliary protein CEP290 (NPHP6) ${ }^{40,41}$ and the ciliary protein lebercilin ${ }^{42}$ are causative for LCA, while mutations in motor protein MyosinVIIa are responsible for Usher syndrome type $1 \mathrm{~B}^{43}$. Aberrations in Retinitis Pigmentosa GTPase Regulator (RPGR) and its interacting protein (RPGRIP1) are causative for $\mathrm{X}$-linked retinitis pigmentosa and LCA, respectively ${ }^{44-48}$. We therefore analyzed the connecting cilium region in detail by immuno-electronmicroscopy (Fig. $5 \mathrm{D}$ and E). Using specific antibodies against whirlin and MPP1, we determined the localization of both proteins in the basal bodies and the connecting cilium itself. In addition, whirlin is detected in the calycal processes surrounding the connecting cilium. 


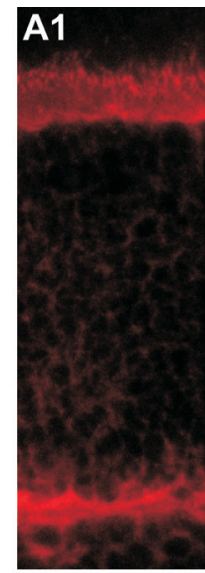

Whirlin

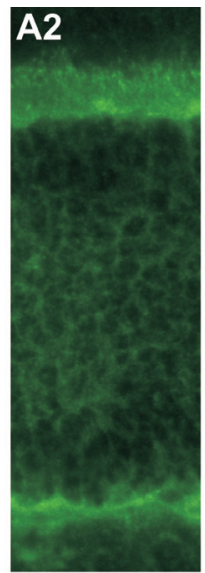

MPP1

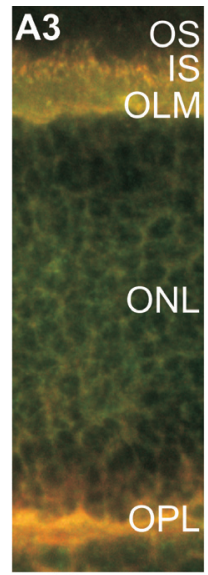

Merged
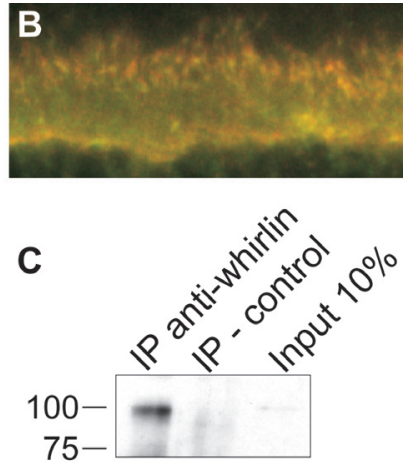

IB: $\alpha$-whirlin
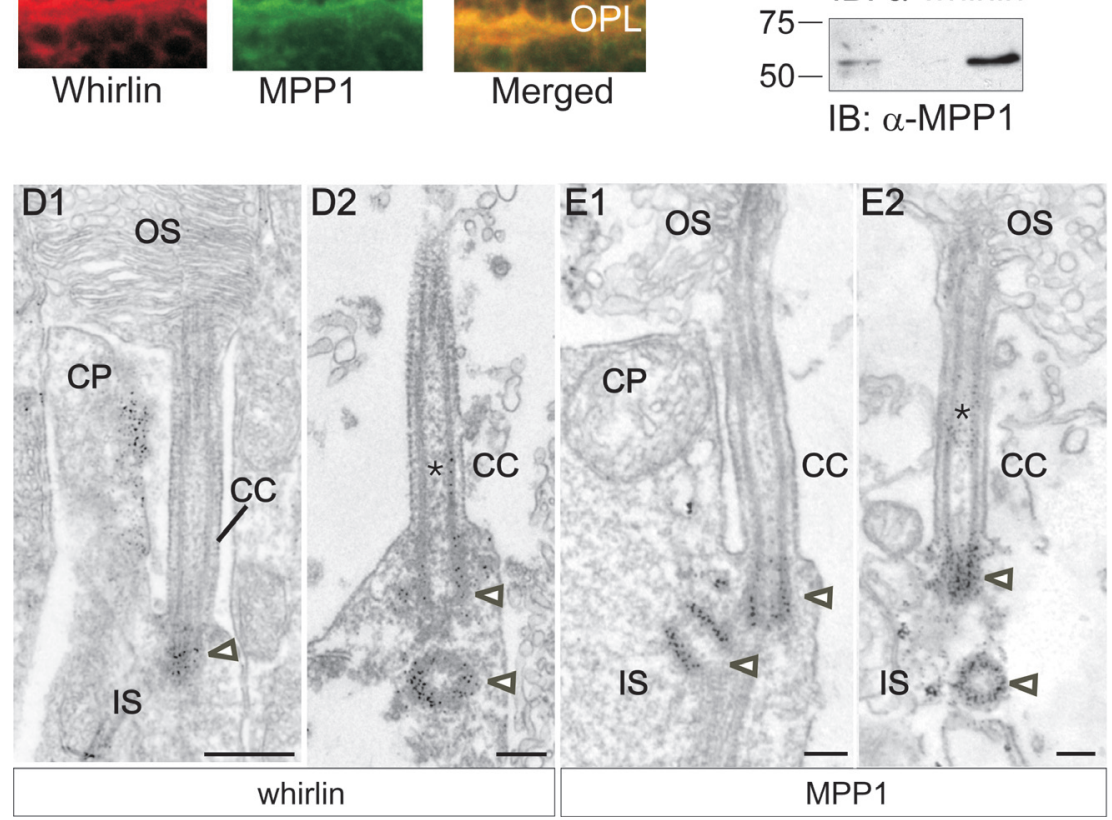

Fig. 5 MPP1 interacts with whirlin in photoreceptors. $(A-B)$. Retinal cryosections were stained using polyclonal antibodies against whirlin $(A 1)$ and MPP1 (A2). The signals show a near-complete overlap in the overlay (A3). (B). The most prominent staining was found at the OPL, OLM and around the inner segments (IS). (C). Immunoprecipitation of whirlin using a polyclonal antiwhirlin antibody precipitated whirlin from mouse retinal lysates (top). MPP1 co-precipitated with anti-whirlin (bottom). As a control, protein $A / G$ agarose beads without primary antibody were used. (D). Pre-embedding immunolabeling of the ciliary region of mouse photoreceptors by antibodies against whirlin shows a clear staining of the calycal processes $(\mathrm{CP})$, the basal bodies (arrow heads) (D1 and D2) and of the connecting cilium (indicated by asterisks) (D2). Sections stained with anti-MPP1 show an identical signal in the basal bodies and the connecting cilium (E1 and E2), but not in the calycal process. Bars in D1 $0.5 \mu \mathrm{m}$; D2 $0.25 \mu \mathrm{m}$; E1 and E2 $0.2 \mu \mathrm{m}$. 


\section{DISCUSSION}

Basic cell polarity and cell adhesion processes that are intimately connected, govern the formation and maintenance of the layered structure of the retina. Although the association with polarity defects is not yet well understood, loss of the correct apico-basal distribution of the associated protein complexes at the cell membranes may trigger the developmental disorganization. In this study, we have assessed one of the key members of the apico-basal polarity complexes, MPP5, for protein-protein interactions that could provide clues for its involvement in retinal patterning. We identified MPP1 as a novel interactor, and by homology modeling predicted a binding mechanism of homo- as well as heterodimerization of the MAGUK modules. Our data indicate that this only occurs in specific orientations of these modules.

MPP1 is known to bind glycophorin $\mathrm{C}$ and protein 4.1 in a complex that facilitates subcortical cytoskeleton-membrane linkage in erythrocytes and has a scaffold function in postsynaptic regions of neurons in the brain ${ }^{49,50}$. A role for this protein in the retina however has never been described. Our findings indicate that MPP1 has several functions in the retina. As a novel member of the Crumbs/MPP5 protein network, it may be a crucial factor in connecting the Crumbs protein complexes and/or the actin cytoskeleton to the membrane, analogous to its function in erythrocytes. Its binding to the transmembrane protein glycophorin- $C$ ${ }^{51}$ and CASK $^{52}$ was not studied here, but may also be needed to provide an accurate docking point to establish specific membrane subcompartments, either in synaptic processes in the OPL or at the OLM.

We demonstrate that the MPP1 gene is ubiquitously expressed, in adult tissues and throughout development. Its expression in the retina showed striking similarities to the retinal expression pattern of DFNB31, the gene encoding whirlin ${ }^{26}$. During development of the retina, expression is highest in the inner, neuroblastic layer, while at later stages it is also present in the photoreceptor cell layer. The increasing expression of whirlin and MPP1 in the inner neuroblastic layer during early development may implicate that these proteins indeed play a role at the early stages of retinal pattern formation.

Multi-PDZ protein whirlin is associated with inherited isolated deafness (DFNB31) ${ }^{27}$ and Usher syndrome (USH2D) ${ }^{28}$. We and other recently identified whirlin as a key player in the Usher protein network both in the retina and in the inner ear ${ }^{26,53}$. As the whirlin-MPP1 interaction was previously identified as a preliminary result from a yeast 
two-hybrid screen, but without further confirmation ${ }^{39}$, we analyzed the direct interaction of whirlin and MPP1. We confirmed the interaction in the yeast two-hybrid system, by GST-pull down analysis and by co-immunoprecipitation from retinal extracts. We could also specify the binding mechanism to a classical PDZ-amino terminal PBM interaction, in combination with a second mechanism, which most likely involves different internal epitopes. Very recently, the MPP1-whirlin interaction was also identified in the inner ear at the stereocilia tip ${ }^{29}$. Our PDZ3-PBM interaction data specify in more detail the PDZ3-GUK interaction that was suggested by Mburu et al. ${ }^{29}$.

Interaction of MPP1 with whirlin may provide a physical connection of the Usher protein network and the Crumbs protein complex at the OLM, via MPP5. A schematic representation of these interactions is given in Figure 6.

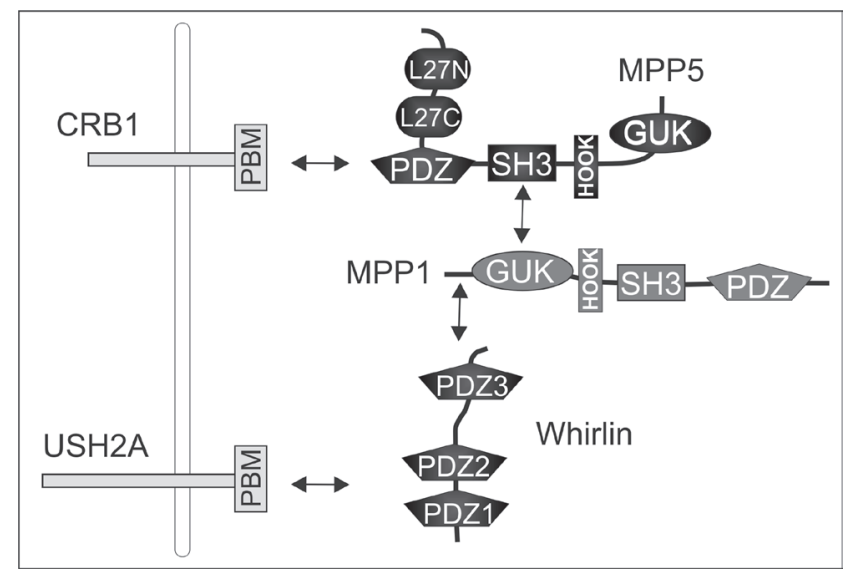

Fig. 6 Schematic representation of the link between the Crumbs and Usher protein networks via MPP1. Transmembrane proteins CRB1 and Usher2A bind with their PDZ binding motifs (PBM) to the PDZ domain of MPP5 and PDZ1/PDZ2 of whirlin, respectively. MPP5 interacts with MPP1 via the SH3GUK domain binding mechanism, while the C-terminus of MPP1 is involved in binding to PDZ3 of whirlin. An additional binding mechanism is involved in the latter which remains to be specified.

It was also previously shown, that other MPP5-associated members of the Crumbs protein complex are present at the OLM ${ }^{18,19}$, as is true for multiple whirlin-associated members of the Usher protein network ${ }^{26,30}$. MPP5 localization is limited to the OLM, whereas MPP1 is also present at the synaptic layer (OPL), the inner segments and the region of the con- 
necting cilium, in a pattern strongly resembling the retinal localization of the Usher syndrome-associated proteins whirlin, USH2A and VLGR-1 ${ }^{26}$. Detailed immuno-EM analysis revealed the presence of both MPP1 and whirlin in the basal bodies and, to a somewhat lesser extent, in the ciliary axoneme of the photoreceptor cilium. We also detected whirlin in the calycal processes of the photoreceptor, the periciliary region. MPP1 was not found at this specific subcellular site. The role of the whirlinMPP1 interaction at the connecting cilium and basal bodies remains to be determined, but a number of findings suggest a role for both proteins in actin organisation and dynamics. Interaction of whirlin with myosin XVa suggests a role in polymerization of the actin filaments that extend beneath the ciliary membrane (similar as in stereocilia development) ${ }^{54,55}$, while association with myosin VIIa provides a link to actin-based opsin transport in the connecting cilia ${ }^{56}$. The known association of MPP1 with $4.1 \mathrm{R}$ and the recently identified colocalisation in stereocilia of the inner ear suggest a role in actin organisation beyond their function in erythrocytes ${ }^{29,57}$. Alternatively or simultaneously, MPP1-whirlin may also contribute to the maintenance of a radial microtubule organization at the basal bodies and connecting cilia by 4.1R, similar to the role of $4.1 \mathrm{R}$ in centrosomes ${ }^{58}$.

The colocalization of MPP1, MPP5 and whirlin in the retina indicates the complementary nature of their interaction. Although the exact roles of these newly identified partnerships in the retina remain to be determined, our current findings suggest a link between the core developmental processes of actin polymerization and cell polarity establishment or maintenance through MPP1. We argue that their interaction, colocalization and coexpression patterns are in line with an important role in generating apico-basal polarity and patterning of the retina, processes that may be disrupted in the inherited disorders associated with defects in the Crumbs and Usher protein networks. 


\section{ACKNOWLEDGEMENTS}

The authors thank E. Sehn for technical assistance. Antibodies were kindly provided by Dr. J. Wijnholds (anti-MPP5 SN47), and Dr. A.H. Chishti (anti-MPP1 serum). This research was supported by grants from the Netherlands Organization for Scientific Research (NWO; grant 912-02018 [to F.P.M.C]), the Algemene Nederlandse Vereniging ter Voorkoming van Blindheid (to R.R. and F.P.M.C), BRPS project GR552 (to H.K. and R.R), EVI-GENORET (LSHG-CT-2005 512036 [to R.R. and F.P.M.C.]), DFG [GRK 1044 (to U.W.)], the FAUN-Stiftung (to U.W.) and the FcBInitiative Usher Syndrom (to U.W. and H.K.). 


\section{SUPPLEMENTARY MATERIAL}

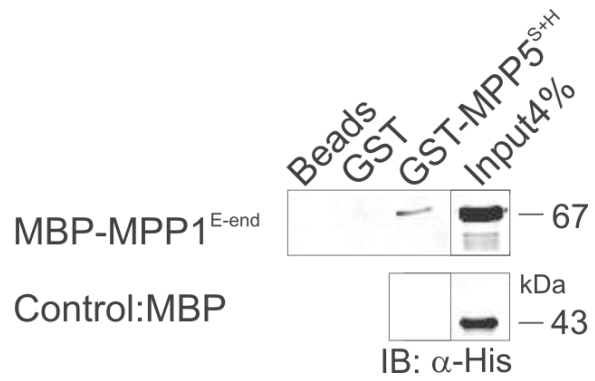

Supplemental fig. 7 GST-pull down MPP1 and MPP5. In a GST-pull down assay, the $\mathrm{SH} 3+\mathrm{HOOK}$ domain of MPP5 pulled down the C-terminal part of MPP1 containing the GUK domain. Beads alone or GST were not able to interact with MPP1, while MPP5 did not bind the MBP tag in the control experiment, showing that this interaction is specific.

28 cycli

$\begin{array}{llllllllllll}1 & 2 & 3 & 4 & 5 & 6 & 7 & 8 & 9 & 10 & 11 & 12\end{array}$

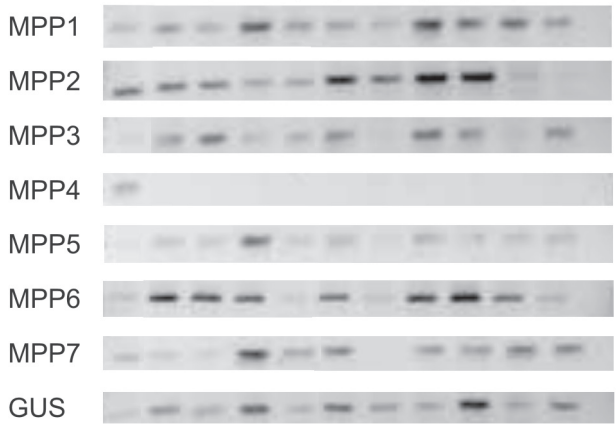

32 cycli

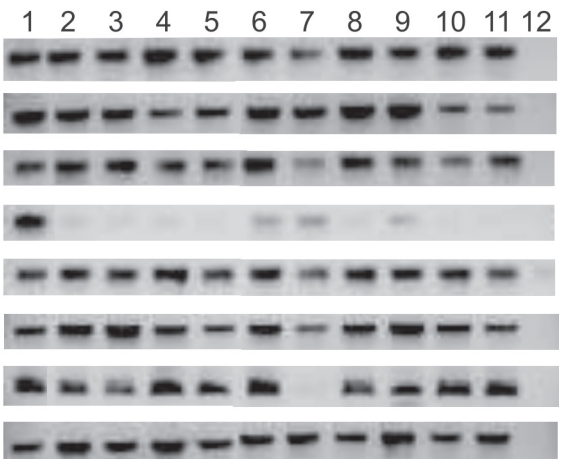

Supplemental fig.8 RT-PCR analysis of the MPP family of genes. Comparison of the expression of MPP1 with the other MPP family members using semi-quantitative RT-PCR analysis of different tissues indicates that MPP1 is most ubiquitously expressed. 1. retina; 2 . fetal cochlea; 3 . fetal brain; 4. kidney; 5. heart; 6. fetal eye; 7. ARPE cell line; 8. brain; 9. testis; 10. skeletal muscle; 11. lung; 12. negative control (water). The housekeeping gene GUS served as a positive control. 


\section{SUPPLEMENTAL MATERIALS AND METHODS}

\section{DNA constructs}

Gene-specific primers that were used to generate MPP1 and whirlin constructs are listed in table 2 . The start/stop codons are underlined and amino acid positions are given between brackets.

Table 2. Sense and antisense primer sequences for Gateway constructs

\begin{tabular}{|c|c|c|}
\hline Construct (a.a. position) & Sense & Antisense \\
\hline $\mathrm{hMPP}^{\mathrm{FL}}(1-466)$ & 5'-ATGACCCTCAAGGCGAG-3' & 5'-AACCCAGGAGACAGGC-3' \\
\hline $\mathrm{hMPP}^{\mathrm{SH} 3+\mathrm{HOOK}}(161-265)$ & 5'-ATGTTCATGAGAGCGCAGTTTG-3' & 5'-CTGATCAAAAATCGAGCTGTG-3' \\
\hline hMPP1 $1^{\text {E-end }}(268-466)$ & 5'-TACGAGGAAGTCGTTCGGC-3' & 5'-TCAGTAAACCCAGGAGACAGGC-3' \\
\hline $\mathrm{hMPP}^{\mathrm{C} \triangle 9}(1-457)$ & 5'-ATGACCCTCAAGGCGAG-3' & 5'-TCATGGAGAACTGCACGCTTGG-3' \\
\hline hMPP1 $1^{\mathrm{GUK}}(281-454)$ & 5'-AAGAGGAAGACCCTGGTGC-3' & 5'-GCACGCTTGGTCGAAGGC-3' \\
\hline hWhirlin ${ }^{\text {PDZ3 }}(561-907)$ & 5'-CTCCCAGATGTGTCCGTG-3' & 5'-GCTAGAGCATCACATTGAACTC-3' \\
\hline
\end{tabular}

\section{Expression profiling}

For MPP gene expression profiling, the following primer pairs were used: 5'-AAGTATGGCTCAGTCAGCTC-3'(sense) and 5'-GGACAGGGTACACAAACTTCT-3' (antisense) forMPP1,5'-AGCGAATGATGTATTTGACC-3' (sense) and 5'-GTCAATACGTGTGCCATACA-3' (antisense) for MPP2. 5'-AAAGAGACCTGTGACTGTGAG-3' (sense) and 5'AAGTGCTGTGGGTTCTCAG -3' (antisense) for MPP3. 5'-GTATCTCACAACCAGAAATGC-3' (sense) and 5'- ACCACATAGGGCATAAACTC-3' (antisense) for MPP6. 5'- GCAAGAAGAGTGATCAGTACG -3' (sense) and 5'- TGAGTCTATACTTGTGCCGTAG -3' (antisense) for MPP7. 5'-GATCCACCTCTCGATGTTCAC-3' (sense) and 5'-CCTTTAGTGTTCCCTGCTAG -3' (antisense) for GUS. 


\section{REFERENCES}

1. Malicki, J. Cell fate decisions and patterning in the vertebrate retina: the importance of timing, asymmetry, polarity and waves. Curr.Opin.Neurobiol. 14, 15-21 (2004).

2. Wei, X. \& Malicki, J. nagie oko, encoding a MAGUK-family protein, is essential for cellular patterning of the retina. Nat.Genet. 150-157 (2002).

3. Malicki, J. et al. Mutations affecting development of the zebrafish retina. Development 123, 263-273 (1996).

4. Malicki, J. \& Driever, W. oko meduzy mutations affect neuronal patterning in the zebrafish retina and reveal cell-cell interactions of the retinal neuroepithelial sheet. Development 126, 1235-1246 (1999).

5. Pujic, Z. \& Malicki, J. Mutation of the zebrafish glass onion locus causes early cell-nonautonomous loss of neuroepithelial integrity followed by severe neuronal patterning defects in the retina. Dev.Biol. 234, 454-469 (2001).

6. Jensen, A. M., Walker, C., \& Westerfield, M. mosaic eyes: a zebrafish gene required in pigmented epithelium for apical localization of retinal cell division and lamination. Development 128, 95-105 (2001).

7. Wei, X., Zou, J., Takechi, M., Kawamura, S., \& Li, L. Nok plays an essential role in maintaining the integrity of the outer nuclear layer in the zebrafish retina. Exp.Eye Res. 83, 31-44 (2006).

8. Omori, Y. \& Malicki, J. oko meduzy and Related crumbs genes are determinants of apical cell features in the vertebrate embryo. Curr.Biol. 16, 945-957 (2006).

9. Horne-Badovinac, S. et al. Positional cloning of heart and soul reveals multiple roles for PKC lambda in zebrafish organogenesis. Curr.Biol. 11, 1492-1502 (2001).
10. Malicki, J., Jo, H., \& Pujic, Z. Zebrafish $\mathrm{N}$-cadherin, encoded by the glass onion locus, plays an essential role in retinal patterning. Dev.Biol. 259, 95-108 (2003).

11. Jensen, A. M. \& Westerfield, M. Zebrafish mosaic eyes is a novel FERM protein required for retinal lamination and retinal pigmented epithelial tight junction formation. Curr.Biol. 14, 711-717 (2004).

12. Laprise, P. et al. The FERM Protein Yurt Is a Negative Regulatory Component of the Crumbs Complex that Controls Epithelial Polarity and Apical Membrane Size. Dev.Cell 11, 363-374 (2006).

13. Bachmann, A., Schneider, M., Theilenberg, E., Grawe, F., \& Knust, E. Drosophila Stardust is a partner of Crumbs in the control of epithelial cell polarity. Nature 414, 638-643 (2001).

14. Knust, E. \& Bossinger, O. Composition and formation of intercellular junctions in epithelial cells. Science 298, 1955-1959 (2002).

15. Hong, Y., Stronach, B., Perrimon, N., Jan, L. Y., \& Jan, Y. N. Drosophila Stardust interacts with Crumbs to control polarity of epithelia but not neuroblasts. Nature 414, 634-638 (2001).

16. Richard, M. et al. Towards understanding CRUMBS function in retinal dystrophies. Hum.Mol.Genet. 15 Spec No 2, R235-R243 (2006).

17. Mehalow, A. K. et al. CRB1 is essential for external limiting membrane integrity and photoreceptor morphogenesis in the mammalian retina. Hum.Mol. Genet. 12, 2179-2189 (2003).

18. Kantardzhieva, A. et al. MPP5 recruits MPP4 to the CRB1 complex in photoreceptors. Invest Ophthalmol.V is.Sci. 46, 2192-2201 (2005). 
19. Van de Pavert, S. A. et al. Crumbs homologue 1 is required for maintenance of photoreceptor cell polarization and adhesion during light exposure. J.Cell Sci. 117, 4169-4177 (2004).

20. Kantardzhieva, A., Alexeeva, S., Versteeg, I., \& Wijnholds, J. MPP3 is recruited to the MPP5 protein scaffold at the retinal outer limiting membrane. FEBS J. 273, 1152-1165 (2006).

21. Kamberov, E. et al. Molecular cloning and characterization of Pals, proteins associated with mLin-7. J.Biol.Chem. 275, 11425-11431 (2000).

22. Hurd, T. W., Gao, L., Roh, M. H., Macara, I. G., \& Margolis, B. Direct interaction of two polarity complexes implicated in epithelial tight junction assembly. Nat.Cell Biol. 5, 137-142 (2003).

23. Roh, M. H. et al. The Maguk protein, Pals1, functions as an adapter, linking mammalian homologues of Crumbs and Discs Lost. J.Cell Biol. 157, 161-172 (2002).

24. Cao, X. et al. PALS1 specifies the localization of ezrin to the apical membrane of gastric parietal cells. J.Biol.Chem. 280, 13584-13592 (2005).

25. McHugh, E. M., Zhu, W., Milgram, S., \& Mager, S. The GABA transporter GAT1 and the MAGUK protein Pals1: interaction, uptake modulation, and coexpression in the brain. Mol.Cell Neurosci. 26, 406-417 (2004).

26. van Wijk, E. et al. The DFNB31 gene product whirlin connects to the Usher protein network in the cochlea and retina by direct association with USH2A and VLGR1. Hum.Mol.Genet. 15, 751-765 (2006).

27. Mburu, P. et al. Defects in whirlin, a PDZ domain molecule involved in stereocilia elongation, cause deafness in the whirler mouse and families with DFNB31. Nat.Genet. 34, 421-428 (2003).
28. Ebermann, I. et al. A novel gene for Usher syndrome type 2: mutations in the long isoform of whirlin are associated with retinitis pigmentosa and sensorineural hearing loss. Hum.Genet. 121, 203-211 (2007).

29. Mburu, P. et al. Whirlin complexes with p55 at the stereocilia tip during hair cell development. Proc.Natl.Acad.Sci. U.S.A 103, 10973-10978 (2006).

30. Reiners, J. et al. Scaffold protein harmonin (USH1C) provides molecular links between Usher syndrome type 1 and type 2. Hum.Mol.Genet. 14, 3933-3943 (2005).

31. Marfatia, S. M., Lue, R. A., Branton, D., \& Chishti, A. H. In vitro binding studies suggest a membrane-associated complex between erythroid p55, protein 4.1, and glycophorin C. J.Biol.Chem. 269, 8631-8634 (1994).

32. Frangioni, J. V. \& Neel, B. G. Solubilization and purification of enzymatically active glutathione S-transferase (pGEX) fusion proteins. Anal.Biochem. 210, 179 187 (1993).

33. Dunn, K. C., Aotaki-Keen, A. E., Putkey, F. R., \& Hjelmeland, L. M. ARPE-19, a human retinal pigment epithelial cell line with differentiated properties. Exp.Eye Res. 62, 155-169 (1996).

34. den Hollander, A. I. et al. Isolation and mapping of novel candidate genes for retinal disorders using suppression subtractive hybridization. Genomics 58, 240-249 (1999).

35. Brandstatter, J. H., Lohrke, S., Morgans, C. W., \& Wassle, H. Distributions of two homologous synaptic vesicle proteins, synaptoporin and synaptophysin, in the mammalian retina. J.Comp Neurol. 370, 1-10 (1996).

36. McGee, A. W. et al. PSD-93 knockout mice reveal that neuronal MAGUKs are not required for development or function of parallel fiber synapses in cer- 
ebellum. J.Neurosci. 21, 3085-3091 (2001).

37. Krieger, E., Koraimann, G., \&

Vriend, G. Increasing the precision of comparative models with YASARA NOVA--a self-parameterizing force field. Proteins 47, 393-402 (2002).

38. Krieger, E. et al. A structural and dynamic model for the interaction of interleukin-8 and glycosaminoglycans: support from isothermal fluorescence titrations. Proteins 54, 768-775 (2004).

39. Yap, C. C. et al. CIP98, a novel PDZ domain protein, is expressed in the central nervous system and interacts with calmodulin-dependent serine kinase. J.Neurochem. 85, 123-134 (2003).

40. Chang, B. et al. In-frame deletion in a novel centrosomal/ciliary protein CEP290/NPHP6 perturbs its interaction with RPGR and results in early-onset retinal degeneration in the rd16 mouse. Hum.Mol.Genet. 15, 1847-1857 (2006).

41. den Hollander, A. I. et al. Mutations in the CEP290 (NPHPG) Gene Are a Frequent Cause of Leber Congenital Amaurosis. Am.J.Hum.Genet. 79, 556-561 (2006).

42. den Hollander, A. I. et al. Mutations in LCA5, encoding the ciliary protein lebercilin, cause Leber congenital amaurosis. Nat.Genet. 39, 889-895 (2007).

43. Weil, D. et al. Human myosin VIIA responsible for the Usher $1 \mathrm{~B}$ syndrome: a predicted membrane-associated motor protein expressed in developing sensory epithelia. Proc.Natl.Acad.Sci.U.S.A 93, 3232-3237 (1996).

44. Hong, D. H., Yue, G., Adamian, M., \& Li, T. Retinitis pigmentosa GTPase regulator (RPGRr)-interacting protein is stably associated with the photoreceptor ciliary axoneme and anchors RPGR to the connecting cilium. J.Biol.Chem. 276, 12091-12099 (2001).

45. Hong, D. H. et al. RPGR isoforms in photoreceptor connecting cilia and the transitional zone of motile cilia. Invest Ophthalmol.Vis.Sci. 44, 2413-2421 (2003).

46. Roepman, R. et al. Positional cloning of the gene for X-linked retinitis pigmentosa 3: homology with the guaninenucleotide-exchange factor RCC1. Hum. Mol.Genet. 5, 1035-1041 (1996).

47. Meindl, A. et al. A gene (RPGR) with homology to the RCC1 guanine nucleotide exchange factor is mutated in X-linked retinitis pigmentosa (RP3). Nat. Genet. 13, 35-42 (1996).

48. Koenekoop, R. K. RPGRIP1 is mutated in Leber congenital amaurosis: a mini-review. Ophthalmic Genet. 26, 175179 (2005).

49. Chishti, A. H. Function of $\mathrm{p} 55$ and its nonerythroid homologues. Curr.Opin. Hematol. 5, 116-121 (1998).

50. Jing-Ping, Z. et al. p55 protein is a member of PSD scaffold proteins in the rat brain and interacts with various PSD proteins. Brain Res.Mol.Brain Res. 135, 204-216 (2005).

51. Marfatia, S. M., Morais-Cabral, J. H., Kim, A. C., Byron, O., \& Chishti, A. H. The PDZ domain of human erythrocyte p55 mediates its binding to the cytoplasmic carboxyl terminus of glycophorin C. Analysis of the binding interface by in vitro mutagenesis. J.Biol.Chem. 272, 24191-24197 (1997).

52. Nix, S. L., Chishti, A. H., Anderson, J. M., \& Walther, Z. hCASK and hDlg associate in epithelia, and their src homology 3 and guanylate kinase domains participate in both intramolecular and intermolecular interactions. J.Biol.Chem. 275, 41192-41200 (2000).

53. Adato, A. et al. Usherin, the defective protein in Usher syndrome type IIA, is likely to be a component of interstereocilia ankle links in the inner ear sensory cells. Hum.Mol.Genet. 14, 3921-3932 (2005). 
54. Delprat, B. et al. Myosin XVa and whirlin, two deafness gene products required for hair bundle growth, are located at the stereocilia tips and interact directly. Hum.Mol.Genet. 14, 401-410 (2005).

55. Belyantseva, I. A. et al. Myosin-XVa is required for tip localization of whirlin and differential elongation of haircell stereocilia. Nat.Cell Biol. 7, 148-156 (2005).

56. Wolfrum, U. \& Schmitt, A. Rhodopsin transport in the membrane of the connecting cilium of mammalian photoreceptor cells. Cell Motil.Cytoskeleton 46, 95-107 (2000).

57. Nunomura, W., Takakuwa, Y., Parra, M., Conboy, J., \& Mohandas, N. Regulation of protein 4.1R, p55, and glycophorin $\mathrm{C}$ ternary complex in human erythrocyte membrane. J.Biol.Chem. 275, 24540-24546 (2000).

58. Perez-Ferreiro, C. M., Vernos, I., \& Correas, I. Protein 4.1R regulates interphase microtubule organization at the centrosome. J.Cell Sci. 117, 6197-6206 (2004). 




\title{
CHAPTER 4
}

\author{
Syntenin-1 is a novel binding partner of \\ cell-polarity determinant MPP5 in the \\ mammalian retina
}

Ilse Gosens ${ }^{1,2}$, Stef J. F. Letteboer ${ }^{1}$, Miriam C. Peeters ${ }^{1}$, Frans P. M. Cremers ${ }^{1,2}$ and Ronald Roepman ${ }^{1,2}$.

${ }^{1}$ Department of Human Genetics and ${ }^{2} N i j m e g e n$ Centre for Molecular Life Sciences, Radboud University Nijmegen Medical Centre, Geert Grooteplein Zuid 10, P.O. Box 9101, 6500 HB, Nijmegen, The Netherlands.

Submitted 



\section{ABSTRACT}

Purpose: The aim of the study was to dissect the binding repertoire of cell polarity determinant MPP5 in the retina. This MAGUK protein with its multiple protein-protein interaction domains recruits a membrane associated protein scaffold and is implicated in the maintenance of cell adhesion between photoreceptors and Müller glia cells.

Methods: Yeast two-hybrid screening of a bovine retinal cDNA library was performed using the HOOK domain of MPP5. Interactions were confirmed in yeast, in GST pull down assays, and by immunoprecipitation. Retinal localizations were determined by immuno-histochemistry.

Results: Syntenin-1 was identified as a binding partner for the HOOK domain of MPP5. Interaction was confirmed between the full length proteins using different biochemical methods. Syntenin-1 co-localized with MPP5 at the outer limiting membrane in the mammalian retina and was also detected in the inner segments around the region of the connecting cilium, the retinal pigment epithelium (RPE) and neuronal cell layers, such as the outer plexiform layer, inner nuclear layer and ganglion cell layer.

Conclusions: We here show that multi-PDZ domain-containing protein syntenin-1 is part of the MPP5 scaffold in the mammalian retina. This is the first report that describes a possible role for syntenin-1 in this tissue. We hypothesize that by interacting with MPP5, syntenin-1 could serve an important function in the macromolecular protein scaffold that is involved in the maintenance of cell adhesion in the mammalian retina. 


\section{INTRODUCTION}

The highly ordered assembly of polarized cells is an essential feature of a functional mammalian retina. The polarized character of different neurons including photoreceptors is manifested by a differential distribution of membrane components, protein complexes and cytoskeletal structures into apical and basolateral compartments. Cellular junctions that separate these compartments allow the targeted uptake and secretion of vesicles, and mediate cell-cell adhesion contributing to retinal integrity ${ }^{1}$.

MPP5 (Membrane Palmitoylated Protein $\underline{5}$ ) is an evolutionary conserved polarity determinant that has a scaffolding function in different polarized cell types. In the mammalian retina, MPP5, also known

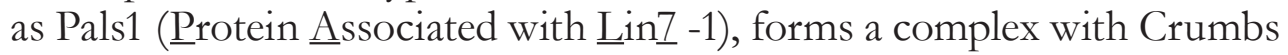
family members ${ }^{2,3}$. Human Crumbs homologue 1 (CRB1) together with MPP5 organizes an intracellular protein scaffold that is thought to be involved in the maintenance of photoreceptor-Müller glia cell adhesion ${ }^{2}$. This macromolecular protein complex is found at the subapical region (SAR), a region just apical to the adherens junctions at the outer limiting membrane (OLM). Adherens junctions are cell-cell junctions formed between photoreceptors and between photoreceptors and Müller glia that share some similarities in compositional build-up as found in tight junctions in epithelia ${ }^{4}$. Mutations in the transmembrane protein CRB1 disrupt the retinal integrity by loss of adhesion causing retinal degeneration in humans ${ }^{5}$. Aberrant MPP5 function in nok zebrafish mutants is associated with retinal patterning defects and loss of OLM integrity ${ }^{6,7}$.

MPP5 belongs to the MAGUK protein family and comprises of several protein-protein interaction domains. The $\mathrm{N}$-terminus consists of two L27 domains and a coiled coil domain followed by the MAGUK module. The C-terminal L27 domain binds to $\operatorname{Lin} 7{ }^{8}$, while the $\mathrm{N}$-terminal L27 domain associates with PATJ ${ }^{9}$. In polarized epithelial cells, MPP5 forms a tripartite complex with human Crumbs homologue 3 (CRB3) and PATJ, and localizes to tight junctions. The MAGUK module consists

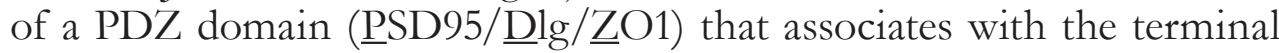
four amino acids of CRB1, CRB2 and CRB3 (ERLI-motif), followed by a SH3 domain ( $\underline{\operatorname{S}} \mathrm{r}$ homology $\underline{3})$. This domain is involved in binding to MPP1, MPP3 and MPP4 protein family members in the retina ${ }^{10-12}$. Furthermore, a region with homology to a guanylate kinase domain (GUK domain) without catalytic activity is present. Some MPP family members, including MPP5, contain a lysine rich region between the SH3 and GUK domain, called the HOOK domain. 
In this study, we describe the identification of a novel interacting partner for the HOOK domain of MPP5 in the retina. A yeast two-hybrid screen of a retinal cDNA library using this HOOK domain as a bait revealed full length syntenin-1 as a binding partner. Additional biochemical methods confirmed the binding of the full length molecules, and by immunohistochemistry we identified colocalization in the retina.

Syntenin-1 (or Pro-TGF-alpha cytoplasmic domain-interacting protein 18, TACIP18 or Melanoma Differentiation-Associated gene 9, MDA9) encodes a protein of 298 amino acids and is composed of three separate structural domains: a tandem of PDZ domains and a relatively large N-terminal domain with unknown function. The protein is an adaptor-like molecule and binds to membrane proteins with structurally related C-termini, for example it binds to the FYA carboxyl terminal amino acids of syndecans ${ }^{13,14}$, neurexins ${ }^{14,15}$ and to the carboxyl terminus of B-type Ephrins ${ }^{14,16,17}$. Syntenin-1 has a variety of functions in different polarized cell types (reviewed in ${ }^{18}$ ), ranging from a role in cytoskeletalmembrane organization ${ }^{13}$, protein trafficking ${ }^{19-21}$ and cell adhesion ${ }^{22}$ to the activation of transcription factors ${ }^{23}$. This study describes a novel function of syntenin-1 in the CRB1-MPP5 associated cell polarity complex in the retina.

\section{MATERIALS AND METHODS}

\section{DNA constructs}

Human retinal cDNA was used to clone full length MPP5 [10]. Full length MPP5, SH3+HOOK, SH3-end (containing the SH3, HOOK and GUK domain) and HOOK domain constructs were made as described previously ${ }^{10}$. Human syntenin-1 was cloned using IMAGEclone \#3853334 and GATEWAY adapted primers were used according to the manufacturer's procedures with start and stop codons underlined (table 1).

\section{Yeast two-hybrid}

A GAL4-based yeast two-hybrid system (Hybrizap, Stratagene, La Jolla, USA) was used to identify proteins that interact with MPP5. A construct containing the DNA binding domain fused to the HOOK domain of MPP5 was transformed in PJ69-4A and used as bait to screen a bovine randomly primed retinal cDNA library (transformed in PJ694 $\alpha$ containing $1.4 \times 10^{6}$ primary clones). Clones were obtained by cell-to-cell mating with an efficiency of $7 \%$, resulting in a total number of $28 \times 10^{6}$ clones on 
selection plates lacking tryptophan, leucine, histidine and adenine. Selection of positive clones was based on yeast growth and activation of $M E L$ 1 and Lac $Z$ reporter genes in an $\alpha$-galactosidase plate assay and $\beta$-galactosidase filter lift assay, respectively ${ }^{24}$. In addition, constructs containing the SH3-end or HOOK domains of MPP5 fused to the DNA binding domain $(\mathrm{pBD})$ or activation domain (pAD) were co-transformed with different constructs of syntenin-1 in PJ694 $\alpha$ yeast cells. The intracellular domain of CRB1 was co-transformed with all syntenin-1 constructs or the PDZ domain of MPP5.

\section{GST pull down, immunoprecipitation and retinal lysates}

IPTG inducible BL21-DE3 cells were transformed with GST-hMPP5 ${ }^{\mathrm{S}+\mathrm{H}}$ / pDest15, GST-hMPP5 ${ }^{\mathrm{HOOK}} / \mathrm{pDest15,}$ GST-Syntenin ${ }^{\mathrm{FL}} / \mathrm{pDest15}$, HisMBP-hMPP5 $5^{\mathrm{s}+\mathrm{H}} / \mathrm{pDest566}$, His-MBP-hMPP5 $5^{\mathrm{HOOK}} / \mathrm{pDest566}$ and HisMBP-Syntenin ${ }^{\mathrm{FL}}$. Bacterial cell lysates were prepared according to a $0.5 \%$ sarkosyl protocol with DNAse added before centrifugation ${ }^{25}$. For pull downs, equal amounts of blocked $(1.5 \mathrm{mg} / \mathrm{ml} \mathrm{BSA})$ glutathione Sepharose 4B beads (Amersham Pharmacia, Roosendaal, The Netherlands) with GST, beads with GST fusion proteins or beads alone were incubated with $1 \mathrm{ml}$ of lysates containing His-MBP-fusion proteins overnight at $4^{\circ} \mathrm{C}$. After several washes with lysis buffer and TBS containing $1 \%$ triton $\mathrm{X}$ 100 and $2 \mathrm{mM}$ DTT, the beads were boiled and proteins were resolved on SDS-PAGE. For Western blotting, proteins were electrophoretically transferred onto nitrocellulose membranes, which were then blocked in 5\% non-fat dry milk (Bio-Rad Laboratories, Utrecht, The Netherlands), incubated with primary antibody anti-His (H-15, Santa Cruz, Inc., Santa Cruz CA, USA) and secondary antibody goat ant-rabbit Alexa680 (Molecular probes, Paisley, USA) in 1\% milk, and washed in PBST $(0.3 \%$ Tween). The bands were visualized using Odyssey® Infrared Imaging system (LI-COR Biosciences, Nebraska, USA).

For immunoprecipitation, HEK293 cells were transfected with full length FLAG-MPP5 using Effectene (Qiagen, Venlo, The Netherlands). After 24 hours, cells were lysed in $250 \mu$ lysis buffer $(50 \mathrm{mM}$ Tris- $\mathrm{HCl} \mathrm{pH} 7.5,150 \mathrm{mM} \mathrm{NaCl}, 0.5 \%$ Triton-X-100 plus Complete protease inhibitors (Roche, Woerden, The Netherlands). Protein A/G beads (Santa Cruz Biotechnology, Inc., Santa Cruz CA, USA) coupled to $5 \mu \mathrm{l}$ of mouse monoclonal anti-syntenin-1 antibody 4D12 (Dr. P. Zimmermann, Leuven, Belgium) were used to immunoprecipitate endogenously expressed syntenin-1 for two hours at $4^{\circ} \mathrm{C}$. To determine the presence of 
FLAG-MPP5 in the co-immunoprecipitate, Western blots were incubated with monoclonal anti-FLAG antibody (F3165, Sigma-Aldrich, Zwijndrecht, The Netherlands). Bands were visualized either using anti-mouse secondary antibodies coupled to IRDye800 (Rockland, Gilbertsville, USA) in combination with the Odyssey ${ }^{\circledR}$ Infrared Imaging system (LICOR Biosciences, Nebraska, USA) or anti-mouse secondary antibodies coupled to HRP and the Supersignal West Pico Chemiluminescent substrate (Pierce, Rockford, USA). Syntenin bands were visualized using the Synaptic Systems antibody and an anti-rabbit secondary antibody coupled to Alexa 680 (Molecular probes, Paisley, UK).

Bovine retinal lysates were prepared as described previously ${ }^{2}$. On Western blot, $20 \mu \mathrm{g}$ of the cytosolic fraction and membrane fraction were used. Syntenin was detected using the mouse monoclonal 4D12 antibody followed by incubation with the anti-mouse secondary antibody coupled to IRDye800 and detection with the Odyssey® Infrared Imaging system.

\section{Animals}

Wistar rats (P20) used for this study were housed under normal conditions with access to food and water ad libitum. All animals were treated in accordance with international and institutional guidelines.

\section{Immunohistochemistry}

Frozen Wistar rat retinal sections of $10 \mu \mathrm{m}$ were treated as described previously ${ }^{26}$ or blocking solution $0.1 \%$ ovalbumin and $0.5 \%$ fishgelatin were used. Sections were incubated overnight with primary antibody and for 1 hour with secondary antibody conjugated to Alexa 488 or 568 (Molecular probes, Paisley, UK). For imaging, a Zeiss Axioscop 2 fluorescence microscope with Axiovision software was used.

\section{Antibodies}

For immunostaining the antibodies in the following concentrations were used: mouse monoclonal anti- $\beta$-catenin, 1:500 (BD Biosciences, Alphen aan de Rijn, The Netherlands); chicken polyclonal anti-MPP5, 1:500 (Dr. J. Wijnholds, Amsterdam, The Netherlands); rabbit polyclonal SySy antisyntenin-1, 1:250 (Synaptic Systems, Goettingen, Germany); rabbit polyclonal anti-syntenin-1, 1:120 (C96, Dr. P. Zimmermann, Leuven, Belgium) and mouse monoclonal anti-acetylated tubulin, 1:500 (Abcam). 


\section{RESULTS}

\section{Identification of syntenin-1}

MPP5 is a scaffold protein comprised of multiple protein-protein interaction domains. To assess potential interacting partners for the HOOK domain of MPP5, a yeast two-hybrid screen with MPP5 ${ }^{\mathrm{HOOK}}$ as a bait was performed (Fig. 1A). We screened a bovine randomly primed retinal cDNA library and identified syntenin-1 as an interactor (Fig. 1B).

Five different syntenin-1 clones were identified. All clones contained the full length coding sequence of syntenin-1 in frame with the GAL4 binding domain and some additional 3' untranslated sequence of variable length. To confirm the interaction, human syntenin-1 was subcloned and different parts as well as the full length construct were tested for interaction with the HOOK domain of MPP5 in yeast two-hybrid assays (Fig. 1B). Of these constructs, full length syntenin-1 was involved in binding to the HOOK domain of MPP5. The two PDZ domains of syntenin have been described to interact with several proteins. However, the first PDZ domain nor the second PDZ domain or both PDZ domains together were involved in binding to MPP5. Also the N-terminus, for which no interacting partners have been identified so far, was not able to bind. Syntenin-1 was also able to interact with the complete C-terminus of MPP5 that contains the HOOK domain in between the SH3 and GUK domain. However, decreased activation of the reporter genes resulting in slower growth on the selection plates and reduced enzymatic activity in the $\alpha$ and $\beta$-galactosidase assays, indicated a reduced affinity compared to a construct that contains only the HOOK domain (Fig. 1C).

Syntenin-1 is known to bind to the C-termini of transmembrane proteins via its PDZ domains. Although syntenin-1 has a preference for binding to a FYA amino acid motif in the intracellular domains, we tested whether it was able to directly bind to CRB1, which has a C-terminal ERLI motif. We tested binding of the PDZ domains as well as full length syntenin-1 to CRB1 $1^{\text {INTRA }}$ in the yeast two-hybrid assay. Syntenin-1 did not bind directly to CRB1, whereas the PDZ domain of MPP5 did (Fig. 1C). 


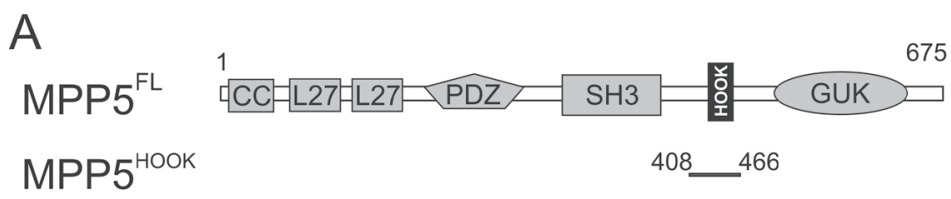

B

bSyntenin- $1^{\mathrm{FL}}$

hSyntenin- $1^{\mathrm{FL}}$

hSyntenin-1 ${ }^{\text {PDZ1 }}$

hSyntenin-1 ${ }^{\text {PDZ2 }}$

hSyntenin- $1^{\mathrm{PDZ1+2}}$

hSyntenin- $1^{\mathrm{N} \text {-ter }}$
675

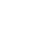

C

\begin{tabular}{|c|c|c|c|}
\hline pBD & pAD & Growth & Coloring \\
\hline hSyntenin- $1^{\mathrm{FL}}$ & hMPP5 $5^{\text {SH3-end }}$ & $+/-$ & $+1-$ \\
\hline hSyntenin- $1^{\mathrm{FL}}$ & hCRB1 $1^{\text {INTRA }}$ & 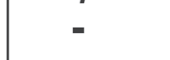 & - \\
\hline hSyntenin-1 $1^{\mathrm{PDZ} 1}$ & hCRB $1^{\text {INTRA }}$ & - & - \\
\hline hSyntenin- $1^{\text {PDZ2 }}$ & hCRB $1^{\text {INTRA }}$ & $=$ & - \\
\hline hSyntenin- $1^{\mathrm{PDZ1+2}}$ & hCRB $1^{\text {INTRA }}$ & - & - \\
\hline$\overline{\mathrm{CRB}} 1^{\text {INTRA }}$ & $\mathrm{MPP}^{\mathrm{PDZ}}$ & ++ & + \\
\hline
\end{tabular}

Fig. 1 Identification of syntenin-1 as interacting partner for MPP5 by a yeast two-hybrid assay. (A). Schematic representation of domain composition of human MPP5. MPP5 consists of a coiled-coiled region (CC), two L27 domains followed by a PDZ, SH3, HOOK and GUK domain. The HOOK domain (a.a. 408-466) fused to the DNA binding domain (BD) was used as a bait to screen a bovine random primed retinal cDNA library. (B). Bovine syntenin-1 was identified from the yeast two-hybrid screen. Full length human syntenin-1 and domains thereof were expressed in yeast in cis with the GAL4 transcription activation domain (AD). Only full length human syntenin-1 interacted with the HOOK domain of MPP5 in yeast, as observed by growth on selection plates and coloring in $\alpha$ - and $\beta$-galactosidase reporter assays. (C). Syntenin- $1^{\mathrm{FL}}$ fused to the GAL4 DNA binding (BD) domain interacted with the C-terminus of MPP5 containing the SH3-HOOK-GUK domain (MPP5 ${ }^{\mathrm{SH} 3-\text { end }}$ ) fused to AD. Neither the PDZ domains nor full length syntenin-1 was able to interact directly with the intracellular domain of CRB1. Interaction between CRB1 ${ }^{\text {INTRA }}$ and the PDZ domain of MPP5 was used as a positive control. 


\section{Confirmation of syntenin-1 binding to MPP5}

Direct interaction between syntenin-1 and MPP5 was confirmed using bacterially expressed proteins in GST pull down assays (Fig. 2A-D). The $\mathrm{HOOK}$ and $\mathrm{SH} 3+\mathrm{HOOK}$ domain of MPP5 fused to GST specifically pulled down syntenin-1 fused to a His-MBP tag, while the control lanes (GST only) were empty (Fig. 2A). Also when the tags were swapped, both MPP5 fusion proteins interacted with syntenin-1 (Fig. 2B). More importantly, full length MPP5 was able to bind to full length syntenin-1 in both orientations (Figs. 2C and D). In addition, a complex of overexpressed FLAG-tagged MPP5 and endogenous syntenin-1 was immunoprecipitated from HEK293 cells (Fig. 2E). Syntenin-1 is endogenously expressed in HEK293 cells (right panel), although the amount that was precipitated by the monoclonal anti-syntenin-1 antibody coupled to protein A/G beads was masked by the presence of heavy chains (left panel). Nevertheless, FLAG-MPP5 that was overexpressed in the HEK293 cells (right panel) was detected in the co-immunoprecipitate using an antiFLAG antibody (left panel). In the control experiment using only protein A/G beads, FLAG-tagged MPP5 was not detected (Fig. 2E).

Endogenous syntenin-1 protein is expressed in the retina. A band around $30 \mathrm{kD}$ is detected in the cytosolic as well as the membrane fraction of freshly prepared bovine lysates (Fig. 2F). 
A

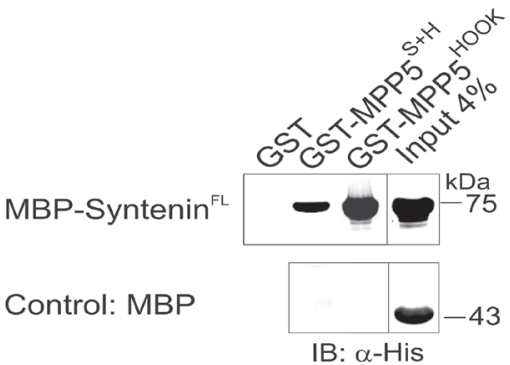

C

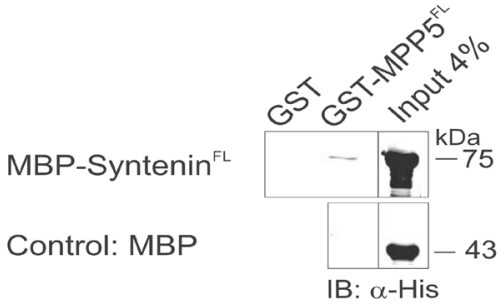

B

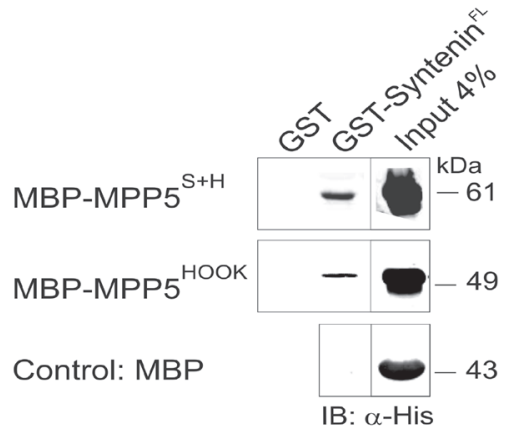

MBP-MPP5 $5^{\text {LL }}$

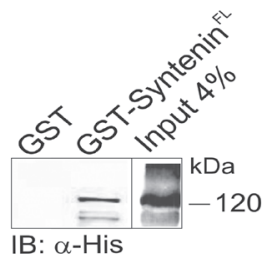

IB: $\alpha-\mathrm{His}$

$\mathrm{F}$

\section{IP anti-Syntenin IP prot $A / G$ beads 3xflag-MPP $5^{\mathrm{FL}}$ Syntenin}

IB: $\alpha$-flag

IB: $\alpha$-syntenin Sysy

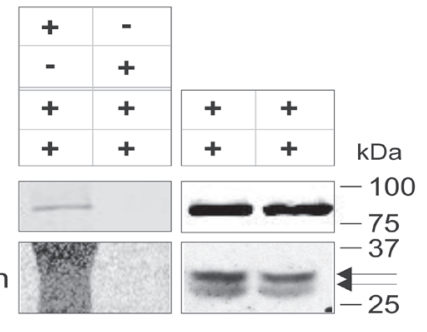

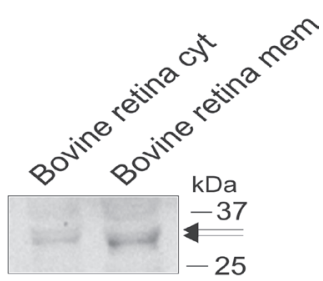

IB: $\alpha$-syntenin 4D12

Fig. 2 Confirmation of the interaction between syntenin-1 and MPP5 by GST pull down assays with bacterially expressed proteins.

(A). The HOOK domain as well as the SH3+HOOK domain of MPP5 fused to GST strongly bind to full length syntenin-1 fused to His-MBP. GST was used as a negative control. In addition, non-specific binding of the His-MBP tag to the GST-MPP5 fusion proteins was not detected. (B). Syntenin-1 interacted with the HOOK and SH3+HOOK domain of MPP5, when GST and His-MBP tags were swapped. (C). Full length syntenin-1 fused to MBP interacts with full length MPP5 fused to GST. All control lanes are empty. (D). Full length MPP5 and syntenin-1 proteins interacted in a tag swap experiment. (E). Overexpressed full length FLAG-tagged MPP5 in HEK293 cells co-immunoprecipitated with endogenously expressed syntenin-1 using a mouse monoclonal antibody against syntenin-1 coupled to protein A/G agarose beads. Protein A/G beads only were used as a negative control. (F). Tissue blot using $20 \mu \mathrm{g}$ of the cytosolic (cyt) and membrane (mem) fraction of bovine retina. Syntenin is detected with the monoclonal 4D12 antibody as a doublet around $30 \mathrm{kD}$. 


\section{Syntenin-1 co-localizes with MPP5 to the OLM}

Although syntenin-1 is found to be important in cell adhesion and protein trafficking in different polarized cell types, its role in the retina has not yet been described. Using two different polyclonal antibodies, we detected similar staining of syntenin-1 in the retinal pigment epithelium (RPE), the outer limiting membrane (OLM), the inner segments, the photoreceptor synaptic layer (outer plexiform layer, OPL), the inner nuclear layer (nuclei of horizontal, bipolar and amacrine neurons, INL) and the neuronal ganglion cell layer (GCL) (Figs. 3A and D). No signal was detected at the outer nuclear layer (ONL) and the inner plexiform layer (IPL). As the rabbit polyclonal SySy antibody detected syntenin-1 in the outer segments (OS), whereas the C96 antibody did not, a putative localization in the OS is not yet conclusive. Co-staining with adherens junction marker $\beta$-catenin revealed an overlapping domain at the OLM, although syntenin-1 signal was also observed apically to this marker. In addition, a small overlap in signals was found at the OPL (Figs. 3A-C). MPP5 signal was found at the SAR as expected, where it coincided with syntenin-1 (Figs. 3D-F). Syntenin-1 was also found more apical to the SAR in the inner segments in a punctuate pattern. Co-staining with ciliary axoneme marker acetylated tubulin was performed to determine a putative connecting cilium staining of syntenin-1. Although the antibodies stainings do not show a complete overlap, the yellow signal in the overlay clearly indicates a localization of syntenin-1 in the region of the connecting cilium (Figs. 3G-I). 

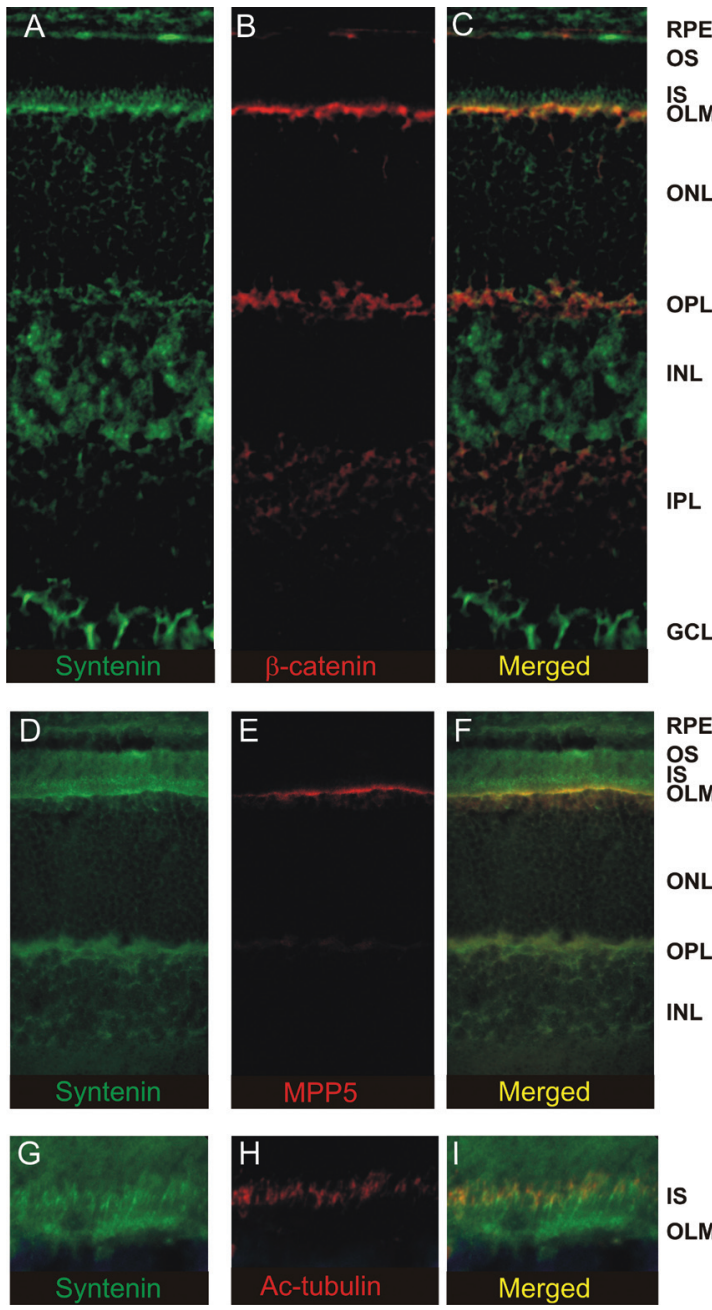

Fig. 3 Localization of syntenin-1 in rat retina.

(A). Syntenin-1 (green) staining using the C96 antibody was found in the retinal pigment epithelium (RPE), inner segments (IS), outer plexiform layer (OPL), outer nuclear layer (ONL), inner nuclear layer (INL) and ganglion cell layer (GCL) and outer limiting membrane (OLM). (B and C). Co-localization with $\beta$-catenin (red), a marker for adherens junctions was found at the OLM. (D). A similar staining pattern for syntenin-1 was observed using the SySy anti-syntenin-1 antibody. (E). MPP5 staining was detected exclusively at the OLM, as previously shown [10]. (F). Co-localization of syntenin-1 (green) with MPP5 (red) was found at the OLM. (G). In the inner segments (IS) a punctate staining pattern of syntenin-1 was observed. (H). Anti-acetylated tubulin antibody, a marker for the ciliary axoneme, stained microtubule fibers of the connecting cilium. (I). Acetylated tubulin and syntenin-1 did not reveal complete overlap, but co-localization was observed at the region of the connecting cilium. 


\section{DISCUSSION}

In this study, we demonstrate a direct association between adaptor molecule syntenin-1 and scaffold protein MPP5 in the retina. Binding involves the HOOK domain of MPP5 and full length syntenin-1. Syntenin-1 is ubiquitously expressed in several tissues, including fetal kidney, liver, lung and brain. High levels of expression have been detected in adult heart and placenta ${ }^{22}$. A role for syntenin-1 in the retina has not yet been described, but the association with polarity determinant MPP5 at the subapical region found in this study, suggests an important role in retinal cell polarity processes.

Studies using polarized mammalian epithelial cells have given important insights into the putative function of syntenin-1 in the retina. In MDCK cells, a model system to study apico-basal cell polarity, syntenin-1 was identified as a component of apical early endocytic vesicles ${ }^{21}$. In addition, syntenin-1 mediates correct targeting of transmembrane molecules to the cell surface, such as pro'TGF ${ }^{19}$. Moreover, deletion of the cytoplasmic tail of syndecan that prevents interaction with syntenin1 retained it in the ER, suggesting a more general role of syntenin-1 in correct targeting of cell surface molecules ${ }^{27}$. Recently, MPP5 itself was found to be involved in correct targeting of CRB1 to the OLM in Müller glia cells ${ }^{28}$, although the molecular mechanism of this process remained elusive. Although we excluded a direct interaction between syntenin-1 and CRB1, syntenin-1 could influence the availability of transmembrane protein CRB1 at the membrane via the endocytotic pathway and mediate its correct targeting via MPP5 in the retina.

Syntenin-1 has been found to co-localize with E-cadherin, $\beta$-catenin an $\alpha$-catenin at cell-cell contacts in epithelial cells and all molecules were found together in a co-immunoprecipitate ${ }^{22}$. Interestingly, the transmembrane protein and structural component of adherens junctions, E-cadherin, is affected by MPP5 depletion. E-cadherin is retained in intracellular puncta, suggesting a role for MPP5 in E-cadherin trafficking to the membrane ${ }^{29}$. In epithelia, MPP5 is important for tight junction formation 9,30. Tight junctions form tight seals between cells and are located apically to adherens junctions. From studies in Drosophila, it was already known that the orthologous complex of Crumbs (CRB1), Stardust (MPP5), and DPATJ (PATJ) localizes apical to adherens junctions, but influences the formation of adherens junctions and E-cadherin by an unknown mechanism ${ }^{31-33}$. This subapical region (also called the marginal zone) is a site that is positionally analogous to the tight junction in 
mammalian epithelia ${ }^{33-37}$. Although in the retina, no tight junctions are formed between the Müller glia and photoreceptors, the adherens junctions, present at the OLM, are their functional analogues. Co-localization of syntenin-1 and MPP5 apical to these junctions provides clues for a similar role for both proteins in cadherin trafficking in the retina.

A direct interaction between syntenin-1 and the E-cadherin/catenin complex has not been identified. However, association of syntenin1 with the cadherin complex could take place via the MPP5-LIN7 interaction. The putative link to the E-cadherin adherens junction complex is shown in figure 4.

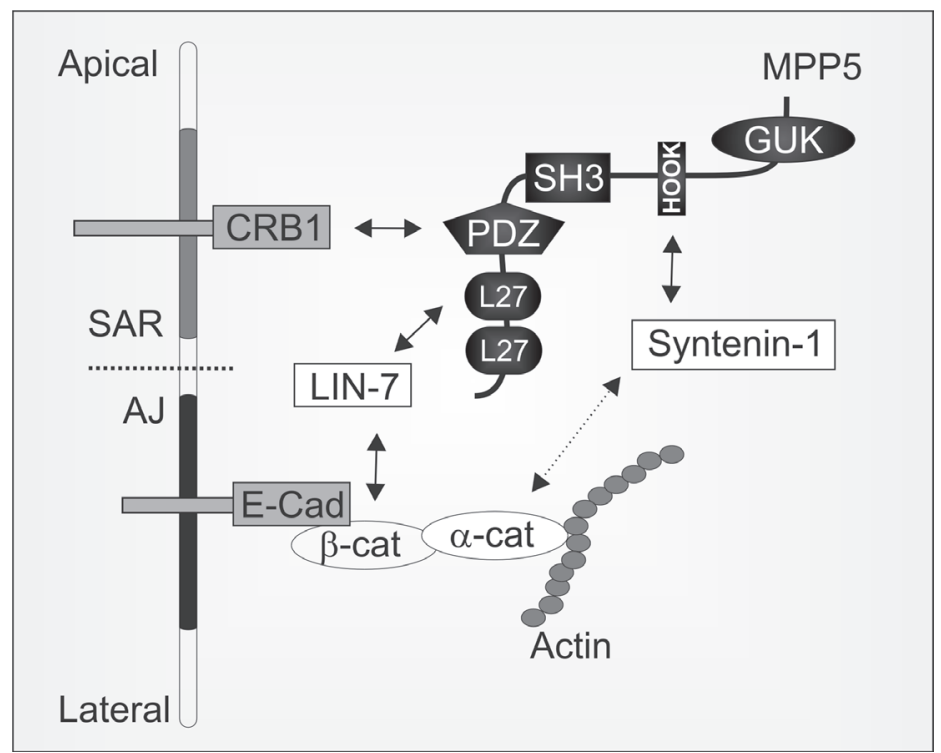

Fig. 4 Illustration of the MPP5-syntenin interaction and the putative link to the E-cadherin $/ \beta$-catenin $/ \alpha$-catenin adherens junction complex. The PDZ domain of MPP5 interacts with transmembrane protein CRB1 at the subapical region (SAR), whereas its C-terminal L27 domain binds to LIN-7. The HOOK domain of MPP5 binds to syntenin-1 (arrows indicate direct interactions). Syntenin-1 was found in a co-immunoprecipitate with adherens junction components $\mathrm{E}$-cadherin, $\beta$-catenin and $\alpha$-catenin (dotted arrow indicates association in a complex). It is feasible that syntenin-1 forms a molecular link between adherens junction molecules and more apically localized proteins via its interaction with MPP5, which in its turn binds to LIN-7.

The heterotrimeric LIN2-LIN7-LIN10 protein network is known to form a complex with cadherin and $\beta$-catenin in epithelia and in neurons in C. elegans. LIN7 binds with its PDZ domain to the C-terminal 
sequence of $\beta$-catenin ${ }^{38}$, while its L27 domain is known to directly bind to the L27 domain of MPP ${ }^{8}$. LIN7, or Veli-3 as it is called in mammals, exists in a complex with MPP5 at the OLM region in the retina, albeit in small amounts ${ }^{39}$. At the region of the OLM in the retina, partial overlap of syntenin-1 with $\beta$-catenin and full overlap with MPP5 suggests that such a relationship is feasible.

Besides localization at the OLM, syntenin-1 was detected at the region of the connecting cilium and in neuronal cells in the outer plexiform layer (OPL), inner plexiform layer (IPL) and ganglion cell layer (GCL), suggesting additional important functions. In neurons in the brain, syntenin- 1 is a binding partner for numerous membrane proteins at pre- and postsynaptic sites ${ }^{15-17}$. It is known to associate with AMPA, kainate and metabotropic glutamate receptors as well as glycine transporter type 2 40-42. In addition, syntenin-1 was found to provide a scaffold for Unc51.1 and the endocytic machinery involved in regulating axon formation in granule cells ${ }^{43}$. A similar scaffolding function of syntenin-1 in retinal neurons can be envisioned.

In conclusion, this study demonstrates for the first time the presence of adaptor molecule syntenin-1 in the retina. Syntenin-1 interacts with polarity determinant MPP5, which is involved in regulation of adhesion between cells at the region of the outer limiting membrane. This interaction provides a molecular link between E-cadherin based cell adhesion and regulation of junction formation in the retina.

\section{ACKNOWLEDGEMENTS}

Antibodies were kindly provided by Dr. J. Wijnholds (anti-MPP5 SN47), and Dr. P. Zimmermann (anti-syntenin C96 and 4D14). This research was supported by grants from the Netherlands Organization for Scientific Research (NWO; grant 912-02-018 to F.P.M.C), the Algemene Nederlandse Vereniging ter Voorkoming van Blindheid (to R.R. and F.P.M.C), EVI-GENORET (LSHG-CT-2005 512036 to R.R. and F.P.M.C.). 


\section{REFERENCES}

1. Malicki, J. Cell fate decisions and patterning in the vertebrate retina: the importance of timing, asymmetry, polarity and waves. Curr.Opin.Neurobiol. 14, 15-21 (2004).

2. Van de Pavert, S. A. et al. Crumbs homologue 1 is required for maintenance of photoreceptor cell polarization and adhesion during light exposure. J.of Cell Sci. 117, 4169-4177 (2004).

3. Mehalow, A. K. et al. CRB1 is essential for external limiting membrane integrity and photoreceptor morphogenesis in the mammalian retina. Hum.Mol.Genet. 12, 2179-2189 (2003).

4. Meuleman, J., Van de Pavert, S. A., \& Wijnholds, J. Crumbs homologue 1 in polarity and blindness. Biochem.Soc.Trans. 32, 828-830 (2004).

5. Richard, M. et al. Towards understanding CRUMBS function in retinal dystrophies. Hum.Mol.Genet. 15 Spec No 2, R235-R243 (2006).

6. Wei, X. \& Malicki, J. nagie oko, encoding a MAGUK-family protein, is essential for cellular patterning of the retina. Nat.Genet. 150-157 (2002).

7. Wei, X., Zou, J., Takechi, M., Kawamura, S., \& Li, L. Nok plays an essential role in maintaining the integrity of the outer nuclear layer in the zebrafish retina. Exp.Eye Res. 83, 31-44 (2006).

8. Kamberov, E. et al. Molecular cloning and characterization of Pals, proteins associated with mLin-7. J.Biol.Chem. 275, 11425-11431 (2000).

9. Roh, M. H. et al. The Maguk protein, Pals1, functions as an adapter, linking mammalian homologues of Crumbs and Discs Lost. J.Cell Biol. 157, 161-172 (2002).

10. Kantardzhieva, A. et al. MPP5 recruits MPP4 to the CRB1 complex in photoreceptors. Invest Ophthalmol.Vis.Sci. 46, 2192-2201 (2005).

11. Kantardzhieva, A., Alexeeva, S., Versteeg, I., \& Wijnholds, J. MPP3 is recruited to the MPP5 protein scaffold at the retinal outer limiting membrane. FEBS J. 273, 1152-1165 (2006).

12. Gosens, I. et al. MPP1 links the Usher protein network and the Crumbs protein complex in the retina. Hum.Mol. Genet. 16, 193-2003 (2007).

13. Grootjans, J. J. et al. Syntenin, a PDZ protein that binds syndecan cytoplasmic domains. Proc.Natl.Acad.Sci.U.S.A 94, 13683-13688 (1997).

14. Grootjans, J. J., Reekmans, G., Ceulemans, H., \& David, G. Synteninsyndecan binding requires syndecan-synteny and the co-operation of both PDZ domains of syntenin. J.Biol.Chem. 275, 19933-19941 (2000).

15. Koroll, M., Rathjen, F. G., \& Volkmer, $H$. The neural cell recognition molecule neurofascin interacts with syntenin-1 but not with syntenin-2, both of which reveal self-associating activity. J.Biol.Chem. 276, 10646-10654 (2001).

16. Lin, D., Gish, G. D., Songyang, Z., \& Pawson, $\mathrm{T}$. The carboxyl terminus of B class ephrins constitutes a PDZ domain binding motif. J.Biol.Chem. 274, 3726-3733 (1999).

17. Torres, R. et al. PDZ proteins bind, cluster, and synaptically colocalize with Eph receptors and their ephrin ligands. Neuron 21, 1453-1463 (1998).

18. Sarkar, D., Boukerche, H., Su, Z. Z., \& Fisher, P. B. mda-9/syntenin: recent insights into a novel cell signaling and metastasis-associated gene. Pharmacol. Ther: 104, 101-115 (2004).

19. Fernandez-Larrea, J., Merlos-Suarez, A., Urena, J. M., Baselga, J., \& Arribas, 
J. A role for a PDZ protein in the early secretory pathway for the targeting of proTGF-alpha to the cell surface. Mol. Cell 3, 423-433 (1999).

20. Zimmermann, P. et al. Syndecan recycling [corrected] is controlled by syntenin-PIP2 interaction and Arf6. Dev. Cell 9, 377-388 (2005).

21. Fialka, I. et al. Identification of syntenin as a protein of the apical early endocytic compartment in Madin-Darby canine kidney cells. J.Biol.Chem. 274, 26233-26239 (1999).

22. Zimmermann, P. et al. Characterization of syntenin, a syndecan-binding PDZ protein, as a component of cell adhesion sites and microfilaments. Mol. Biol.Cell 12, 339-350 (2001).

23. Geijsen, N. et al. Cytokine-specific transcriptional regulation through an IL-5Ralpha interacting protein. Science 293, 1136-1138 (2001).

24. Roepman, R., Schick, D., \& Ferreira, P. A. Isolation of retinal proteins that interact with retinitis pigmentosa GTPase regulator by interaction trap screen in yeast. Methods Enzymol. 316, 688-704 (2000).

25. Frangioni, J. V. \& Neel, B. G. Solubilization and purification of enzymatically active glutathione S-transferase (pGEX) fusion proteins. Anal.Biochem. 210, 179187 (1993).

26. van Wijk, E. et al. The DFNB31 gene product whirlin connects to the Usher protein network in the cochlea and retina by direct association with USH2A and VLGR1. Hum.Mol.Genet. 15, 751-765 (2006).

27. Miettinen, H. M., Edwards, S. N., \& Jalkanen, M. Analysis of transport and targeting of syndecan-1: effect of cytoplasmic tail deletions. Mol.Biol.Cell 5, 1325-1339 (1994).

28. van Rossum, A. G. et al. Pals1/Mpp5 is required for correct localization of Crb1 at the subapical region in polarized Muller glia cells. Hum.Mol.Genet. 15, 2659-2672 (2006).

29. Wang, Q., Chen, X. W., \& Margolis, B. PALS1 Regulates E-Cadherin Trafficking in Mammalian Epithelial Cells. Mol.Biol.Cell 18, 874-885 (2007).

30. Straight, S. W. et al. Loss of PALS1 expression leads to tight junction and polarity defects. Mol.Biol.Cell 15, 19811990 (2004).

31. Grawe, F., Wodarz, A., Lee, B., Knust, E., \& Skaer, H. The Drosophila genes crumbs and stardust are involved in the biogenesis of adherens junctions. Development 122, 951-959 (1996).

32. Tepass, U. Crumbs, a component of the apical membrane, is required for zonula adherens formation in primary epithelia of Drosophila. Dev.Biol. 177, 217-225 (1996).

33. Klebes, A. \& Knust, E. A conserved motif in Crumbs is required for E-cadherin localisation and zonula adherens formation in Drosophila. Curr.Biol. 10, 76-85 (2000).

34. Bachmann, A., Schneider, M., Theilenberg, E., Grawe, F., \& Knust, E. Drosophila Stardust is a partner of Crumbs in the control of epithelial cell polarity. Nature 414, 638-643 (2001).

35. Hong, Y., Stronach, B., Perrimon, N., Jan, L. Y., \& Jan, Y. N. Drosophila Stardust interacts with Crumbs to control polarity of epithelia but not neuroblasts. Nature 414, 634-638 (2001).

36. Medina, E. et al. Crumbs interacts with moesin and betaheavy-spectrin in the apical membrane skeleton of Drosophila. J.Cell Biol 158, 941-951 (2002).

37. Tepass, U. Adherens junctions: new insight into assembly, modulation and function. Bioessays 24, 690-695 (2002).

38. Perego, C., Vanoni, C., Massari, S., 
Longhi, R., \& Pietrini, G. Mammalian LIN-7 PDZ proteins associate with beta-catenin at the cell-cell junctions of epithelia and neurons. EMBO J.19, 3978-3989 (2000).

39. Stohr, H. et al. Membrane-associated guanylate kinase proteins MPP4 and MPP5 associate with Veli3 at distinct intercellular junctions of the neurosensory retina. J.Comp Neurol. 481, 31-41 (2005).

40. Hirbec, H. et al. The PDZ proteins PICK1, GRIP, and syntenin bind multiple glutamate receptor subtypes. Analysis of PDZ binding motifs. J.Biol.Chem. 277, 15221-15224 (2002).

41. Hirbec, H. et al. Rapid and differential regulation of AMPA and kainate receptors at hippocampal mossy fibre synapses by PICK1 and GRIP. Neuron 37, 625-638 (2003).

42. Ohno, K. et al. The neuronal glycine transporter 2 interacts with the PDZ domain protein syntenin-1. Mol.Cell Neurosci. 26, 518-529 (2004).

43. Tomoda, T., Kim, J. H., Zhan, C., \& Hatten, M. E. Role of Unc51.1 and its binding partners in CNS axon outgrowth. Genes Dev. 18, 541-558 (2004). 



\section{CHAPTER 5}

\section{FERM protein EPB41L5 is a novel member of the mammalian CRB-MPP5 polarity complex}

Ilse Gosens ${ }^{1,2}$, Alessandro Sessa ${ }^{3}$, Anneke I. den Hollander ${ }^{1,2}$, Stef J. F. Letteboer ${ }^{1}$, Valentina Belloni ${ }^{3}$, Maarten L. Arends ${ }^{1}$, André Le Bivic ${ }^{4}$, Frans P. M. Cremers ${ }^{1,2}$, Vania Broccoli ${ }^{3}$ and Ronald Roepman ${ }^{1,2}$.

${ }^{1}$ Department of Human Genetics and ${ }^{2}$ Nijmegen Centre for Molecular Life Sciences, Radboud University Nijmegen Medical Centre, Geert Grooteplein Zuid 10, P.O. Box 9101, 6500 HB, Nijmegen, The Netherlands

${ }^{3}$ Stem Cell Research Department (SCRI), San Raffaele Scientific Institute, Via Olgettina 58, 2013, Milan, Italy

${ }^{4} U M R$ CNRS 6216, Institute of Developmental Biology of Marseille-Luminy, Faculté des Sciences de Luminy, Case 907, 13288 Marseille Cedex 09, France

Published in Experimental Cell Research (2007)

Nov 15;313(19):3959-3970. 



\section{ABSTRACT}

Cell polarity is induced and maintained by separation of the apical and basolateral domains through specialized cell-cell junctions. The Crumbs protein and its binding partners are involved in formation and stabilization of adherens junctions. In this study, we describe a novel component of the mammalian Crumbs complex, the FERM domain protein EPB41L5, which associates with the intracellular domains of all three Crumbs homologues through its FERM domain. Surprisingly, the same FERM domain is involved in binding to the HOOK domain of MPP5/ PALS1, a previously identified interactor of Crumbs. Co-expression and co-localization studies suggested that in several epithelial derived tissues Epb4.115 interacts with at least one Crumbs homologue, and with Mpp5. Although at early embryonic stages Epb4.115 is found at the basolateral membrane compartment, in adult tissues it co-localizes at the apical domain with Crumbs proteins and Mpp5. Overexpression of Epb4.115 in polarized MDCK cells affects tightness of cell junctions and results in disorganization of the tight junction markers ZO-1 and PATJ. Our results emphasize the importance of a conserved Crumbs-MPP5-EPB41L5 polarity complex in mammals. 


\section{INTRODUCTION}

Cell polarity is a critical feature of many animal cells and is important for several biological events, such as correct formation and function of epithelia as well as asymmetrical localization of membrane components and cytoskeletal structures. In epithelial cells and neuronal cells of epithelial origin such as photoreceptors, polarity is accomplished by separation of the apical and basolateral domains through specialized cell-cell junctions.

Different protein complexes have been implicated in establishing and maintaining cell polarity, such as the transmembrane protein Crumbs, and its binding partners MPP5/PALS1 and PATJ. In mammals, three Crumbs homologues with highly conserved intracellular domains are known. While mutations in human Crumbs homologue 1 (CRB1) are implicated in retinal dystrophies (Reviewed in ${ }^{1}$ ), not much is known about the function of CRB2. CRB3 is expressed in several (epithelial) tissues and is involved in recruiting protein complexes that form tight junctions at the membrane ${ }^{2}$.

In epithelial tissues of Drosophila, the homologous complex of Crumbs-Stardust and DPATJ localizes to the subapical region (SAR), a region which harbors similar protein complexes as vertebrate tight junctions ${ }^{3}$. This complex is involved in the formation and stabilization of the adherens junctions, and thereby maintaining polarity in embryonic epithelia ${ }^{4-8}$. It is also involved in the morphogenesis of photoreceptors as mutants that do not express Crumbs or Stardust in photoreceptors have shorter stalk membranes ${ }^{9,10}$. Overexpression of Crumbs results in an expansion of the apical membrane in embryonic epithelia and stalk membranes in photoreceptors ${ }^{11,12}$.

In the vertebrate eye, Crb1 is required for maintenance of adherens junctions in the zonula adherens (ZA), while it localizes just apical to this region, also known as the outer limiting membrane. A discontinuous and fragmented ZA was observed in Crb1 mutant mouse retinas during photoreceptor morphogenesis and light dependent retinal degeneration ${ }^{13,14}$. Defects in CRB1 expression lead to abnormal retinal layering, as seen by immature lamination development in humans with retinal degeneration ${ }^{15}$. In zebrafish mutants nagie oko (nok) and mosaic eyes (moe), mutations in MPP5/PALS1 and EPB41L5 respectively, disrupt retinal layering ${ }^{16-18}$. Mosaic eyes mutants also lack tight junction formation in the retinal pigment epithelium (RPE) ${ }^{19}$. 
The EPB41L5 protein is part of the protein 4.1 super family ${ }^{20}$. The prototypical member, EPB41 or protein 4.1R, has a structural function in red blood cells by linking the cytoskeleton to the transmembrane protein Glycophorin C ${ }^{21,22}$. EPB41L5, like all EPB41-related proteins, contains a FERM domain (4.1/Ezrin/radixin/moesin). Crumbs proteins have a conserved motif (juxtamembrane motif) that contains a FERM domain-binding site ${ }^{8}$, besides the conserved carboxy terminal ERLI motif that binds to the PDZ domain of MPP5/Stardust. MAGUK protein MPP5 (membrane palmitoylated protein 5) consists of two L27 domains, an SH3, a GUK and a HOOK domain. The HOOK domain contains a lysine rich region (FERM domain binding motif) that hypothetically could bind to FERM domain proteins ${ }^{23}$.

In this study we determined the localization of Epb4.115 protein in embryonic and adult tissues. Co-expression of Crb1, Crb2, Crb3, Epb4.115 and $M p p 5$ in several tissues as well as co-localization of the proteins at the apical domain in kidney epithelial cells and in the retina led us to study the involvement of Epb4.115 in the mammalian Crumbs/Mpp5 polarity complex. Our studies confirmed the interaction between the FERM domain of EPB41L5 to Crumbs, and we show a direct interaction between MPP5 and EPB41L5. Functionally, overexpression of Epb4.115 in a polarized MDCK cell model has an effect on the formation of tight junctions.

EPB41L5 homologues have been identified in Drosophila and zebrafish ${ }^{19,24}$. Very recently, two reports described the involvement of Yurt and YMO1 in the Crumbs complex ${ }^{25,26}$. Yurt and YMO1 were suggested to act as a negative regulator of Crumbs protein function, thereby regulating apical membrane growth in epithelia and photoreceptors. In addition, interaction data on Yurt/YMO1 binding to members of the Crumbs complex (CRB1 and MPP5) were presented. Here, we show that EPB41L5 binds to all Crumbs homologues and to MPP5, thereby confirming previous findings. In addition, we provide a detailed analysis of the co-expression and co-localisation analysis of Crumbs, MPP5 and EPB41L5 in different mouse tissues. Our findings confirm the evolutionary conserved importance of EPB41L5 in the mammalian Crumbs protein complex in different cell types and point towards a regulating function in apico-basal cell polarity. 


\section{MATERIALS AND METHODS}

\section{DNA constructs}

Human retinal cDNA was used to clone the intracellular domain of $C R B 1$ and full length MPP5 ${ }^{27}$. Full length EPB41L5 and EPB41 were cloned from IMAGEclones \#5502663 and \#5167140 respectively. Human retinal cDNA (marathon cDNA, Clontech) and GATEWAY cloning technology (Invitrogen) were used to clone different expression constructs of CRB2, CRB3, EPB41L5, MPP5, EPB41L4B, MOESIN and EPB41. The GATEWAY adapted primers with gene-specific sequences and amino acid positions that are included in the constructs are listed in table S1 (supplementary data). The attB1 and attB2 linkers were attached to the 5'-end of respectively sense and antisense primers according to manufacturer's procedures. The start and stop codon are underlined.

PCR products were cloned into $\mathrm{PDONR}$ vectors by homologous recombination and verified by nucleotide sequencing ${ }^{27}$. The subsequent ENTRY clones were transferred into several GATEWAY-adapted destination vectors, pBD-GAL4/DEST, pAD-GAL4/DEST, pDest566 ${ }^{27}$, pSOS and pMyr. Destination vectors pDest-15 (N-GST fusion tag), p3xflag-CMV/ Dest and pcDNA3-HA/Dest were purchased from Invitrogen.

\section{Yeast two-hybrid}

A GAL4-based yeast two-hybrid system (Hybrizap, Stratagene) was used. Constructs of the intracellular domain of CRB1, CRB2 or CRB3 fused to the DNA binding domain ( $\mathrm{pBD}$ ) or activation domain ( $\mathrm{pAD}$ ) were co-transformed in PJ694 $\alpha$ yeast cells together with different constructs of EPB41L 5, EPB41, EPB41L4B and MOESIN. Clones positive for interaction were selected based on growth on selection plates lacking tryptophan, leucine and histidine, and on activation of the MEL-1 reporter gene, as seen by blue coloring in an $\alpha$-galactosidase plate assay.

The SOS recruitment (Cytotrap) system was purchased from Stratagene. Yeast strain cdc $25 \mathrm{H}$ was co-transformed with constructs pMyrEPB41L5 ${ }^{\mathrm{FL}}, \mathrm{pMyr}-\mathrm{EPB} 41 \mathrm{~L} 5^{\mathrm{FERM} \_A B C}, \mathrm{pMyr}-\mathrm{EPB} 41 \mathrm{~L} 5^{\mathrm{FERM}_{-} \mathrm{BC}}$ or $\mathrm{pMyr}-$ MPP5 ${ }^{\mathrm{PDZ}}$ separately, together with pSOS-CRB1 ${ }^{\text {INTRA }}$. The FERM domain and full length construct of EPB41L5 fused to pSOS were excluded from the assay, since activation of the Ras signal transduction was seen without recruitment to the plasma membrane by interaction with proteins fused to pMyr. This was verified by determining growth of yeast on glucose plates (no pMyr expression by repression of galactose driven promotor). Protein-protein interactions were determined by growth on galactose 
plates at $37^{\circ} \mathrm{C}$.

\section{GST-pull down and immunoprecipitation}

IPTG inducible BL21-DE3 cells were transformed with GST-CRB1 ${ }^{\text {INTRA } / p D e s t 15, ~ G S T-C R B 1 ~}{ }^{\text {INTRAAGTY } / p D e s t 15, ~ G S T-C R B 1 ~} 1^{\text {INTRA } L E R L I} /$ pDest15, GST-EPB41L5 5L/pDest15, GST-EPB41L5 ${ }^{\text {FERM_ABC } / p D e s t 15, ~}$ GST-EPB41L5 ${ }^{\text {FERM_BC } / p D e s t 15, ~ H i s-M B P-C R B 1 ~}{ }^{\text {INTRA }} /$ pDest566, HisMBP-EPB41L5 5L/pDest566, His-MBP-EPB41L5 ${ }^{\text {FERM_ABC } / p D e s t 566 ~ o r ~}$ His-MBP-EPB41L5 5ERM_BC/pDest566. Cell lysates were prepared according to a $0.5 \%$ sarkosyl protocol ${ }^{28,29}$. GST-pull down was performed as described previously ${ }^{30}$. Alternatively, GST fusion proteins were incubated with radioactive labeled in vitro translated Epb4.115.

Bovine retinas obtained from the slaughterhouse were used for GST pull down assays. Cytosolic and membrane fractions were prepared as described previously ${ }^{14}$. The membrane fraction of one retina was incubated for three hours with GST, GST-CRB1 ${ }^{\text {INTRA }}$, CRB1 ${ }^{\text {INTRA } \triangle G T Y}$ or CRB1 ${ }^{\text {INTRAAERLI }}$. After several washes, beads were boiled and proteins were resolved on SDS-PAGE. For Western blotting, chicken anti-MPP5 (1:500, Dr. J. Wijnholds), guinea pig anti-EPB41L5 (1:10.000), secondary antibodies coupled to HRP and the Supersignal West Pico Chemiluminescent substrate from Pierce were used.

For immunoprecipitation of Crb3 and Epb4.115, stably transfected MDCK cells expressing p75-Crb3 and p75-Crb3 2 ERLI, generated as described previously, were transfected with myc-Epb4.115 ${ }^{31}$. P75-Crb3 and $\mathrm{p} 75-\mathrm{Crb} 3 \triangle \mathrm{ERLI}$ chimeras were used to circumvent the absence of recognition by the anti-Crb3 antibody that was raised against the $\mathrm{C}$-terminus. MDCK cells expressing hCRB3 and p75-Crb3 show identical apical localization ${ }^{31}$. Mouse monoclonal antibodies against human p75NTR (ME 20-4) ${ }^{32}$, or myc (BD Bioscience) were used for immunoprecipitation coupled to protein A/G Plus agarose beads (Santa Cruz Biotechnology). For immunoprecipitation of MPP5 and EPB41L5, HEK293 cells were co-transfected with full length 3xflag-EPB41L5 and HA-MPP5. AntiEPB41L5 was coupled to beads for 2 hours at $4^{\circ} \mathrm{C}$ and washed 4 times with lysisbuffer (50 mM Tris $\mathrm{pH} 7.5,150 \mathrm{mM} \mathrm{NaCl}$ and $0.5 \%$ Triton$\mathrm{X}-100)$. Cells were lysed and added to pre-coupled beads. Immunoprecipitation was performed for 2 hours at $4^{\circ} \mathrm{C}$. As a control, guinea pig IgGs (Rockland) coupled to agarose A/G beads were used. Precipitated proteins were washed 4 times with lysis buffer and analyzed by Western blotting. A polyclonal anti-flag antibody (Sigma-Aldrich) or monoclonal 
anti-HA antibody (Sigma-Aldrich) and secondary antibodies, goat antirabbit 680 Alexa or goat anti-mouse 680 (Molecular Probes) were used.

\section{Animals}

Wistar rats and guinea pigs (Harlan, The Netherlands) used for this study were housed under normal conditions with access to food and water ad libitum. CD1 outbred mice were used to obtain staged embryos and adult organ tissues for whole mount in situ hybridization and immunohistochemistry, respectively. All animals were treated in accordance with international and institutional guidelines.

\section{Immunohistochemistry on tissue}

E15.5 mouse embryos were isolated and fixed in 4\% paraformaldeyde in PBS overnight. Embryos were subsequently frozen in OCT embedding medium and cut to $10 \mu \mathrm{m}$ cryosections. Adult kidneys were isolated from previously perfused adult animals (8 weeks old) and frozen in OCT before slicing. Sections were treated with blocking solution $(0.1 \%$ Triton, 10\% Donkey Serum in PBS) for 2 hours at room temperature and incubated with primary and secondary antibodies diluted in blocking solution. Slides were mounted with Fluorescent mounting medium (DAKO) and processed for imaging with an Eclypse E600 microscope equipped with fluorescent light source (Nikon). Unfixed eyes of Wistar rats (P20) were isolated and frozen in melting isopentane. Immunohistochemistry was performed as described previously ${ }^{33}$.

\section{MCDK cell culture and clonal selection}

MDCK cells were transfected with a CMV-Epb4.115-ires-Neomycin construct using Lipofectamin 2000 reagent according to the manufacturer's instructions (Invitrogen). MDCK cells were selected for resistance to G418 (350 $\mu \mathrm{g} / \mathrm{ml})$. Single clones were picked after 3 weeks and expanded before testing transgene expression by Western blotting.

\section{Calcium switch and immunohistochemistry}

Approximately $5 \times 10^{5} \mathrm{MDCK}$ cells were seeded into $10 \mathrm{~mm}$ diameter Transwell membrane filters $(0.4 \mu \mathrm{m}$ pore size; Corning Costar) and grown to confluence in normal calcium medium. Filters were washed three times with PBS and grown in low calcium media containing 5\% dialyzed FBS and $5 \mu \mathrm{M} \mathrm{Ca}^{2+}$ overnight to dissociate cell-cell contacts. The next day, the low calcium media was replaced with pre-warmed normal calcium media 
and this was designated as the $\mathrm{t}=0$ time point.

Immunohistochemistry was performed as described previously with some modifications ${ }^{34}$. Briefly, MDCK cells grown on filters were fixed in 4\% formaldehyde/PBS for 20 minutes and permeabilized with a solution of gelatin $(0.7 \%)$ saponin $(0.016 \%)$ for 15 minutes at $37^{\circ} \mathrm{C}$. After blocking the filters with $2 \%$ donkey serum/PBS for 1 hour at room temperature, filters were excised and incubated with primary antibodies in $2 \%$ donkey serum (rabbit anti-PATJ, mouse anti-ZO-1, mouse anti$\beta$-catenin) for 3 hours at $37^{\circ} \mathrm{C}$ in a humidified chamber. After extensive washes with $2 \%$ donkey serum/PBS, filters were incubated with fluorochrome-conjugated secondary antibody $(1: 1,000)$ for $1-2$ hours at $37^{\circ} \mathrm{C}$. Subsequently, filters were washed several times with PBS and mounted on glass slides with the Fluorescent mounting medium (Dako). Immunofluorescence was analyzed using an Eclipse E600 (Nikon) and a TCS SP2 Laser Scanning Confocal (Leica) microscopes.

\section{Whole Mount embryos in situ hybridization (WISH)}

RNA probes labeled with Digoxigenin-rUTP (Boehringer Mannheim) were synthesized from linearized cDNA templates according to manufacturer's instructions. The following templates were used: a 550 bp Crb1 3' coding region, an 822 bp Crb2 coding region, and full-length coding regions of Epb4.115, Mpp5 and Crb3.

WISH was performed as described previously with modifications 35. Embryos were fixed for 30 minutes at room temperature in $4 \%$ paraformaldehyde in PBS and treated for 8-15 minutes with $1 \mu \mathrm{g} / \mathrm{ml}$ proteinase $\mathrm{K}$ in $1 \mathrm{mM}$ EDTA, $20 \mathrm{mM}$ Tris- $\mathrm{HCl}$ (pH 7.0). Hybridization and subsequent washes were performed at $58^{\circ} \mathrm{C}$. A 1:2000 dilution of antidigoxigenin-AP conjugated antibody (Roche) was used. Embryos were incubated with the antibody overnight at $4^{\circ} \mathrm{C}$, washed for 24 hours in TBST, for 30 minutes in NTM-T $\left(0.1 \mathrm{M} \mathrm{NaCl}, 50 \mathrm{mM} \mathrm{MgCl}_{2}, 0.1 \mathrm{M}\right.$ Tris$\mathrm{HCl}, \mathrm{pH} 9.5,0.1 \%$ Tween-20), and stained using centrifuged $\mathrm{BM}$ purple AP substrate (Roche) in $0.3 \%$ Tween-20 for $12-36$ hour at $4^{\circ} \mathrm{C}$ or room temperature. They were washed in NTMT, then in distilled water, and stored in $4 \%$ paraformaldeyde at $4^{\circ} \mathrm{C}$ for following analysis.

\section{Antibodies}

Antibodies against purified recombinant GST-hEPB41L5 (acc no: NP_ 065960, a.a. 476-733) were raised in guinea pigs. Serum was affinity purified using HiTrap columns according to manufacturer's procedures 
(Amersham Biosciences).

For immunostaining the following antibodies were used: anti- $\beta$-catenin (Transduction laboratories), anti-MPP5 (Dr. J. Wijnholds), anti-ZO1 (Zymed), anti-Crb1 (AK2, Dr. J. Wijnholds), anti-Crb2 (Dr. A. Le Bivic), anti-Crb3 (Dr. A. Le Bivic), anti-PATJ (Dr. A. Le Bivic), E-cadherin (BD, Bioscience). Secondary antibodies were conjugated with Alexa 488 or Alexa 568 (Molecular probes, Leiden, the Netherlands).

\section{RESULTS}

\section{Overlap of Epb4.115 expression with CRBs and Mpp5 in mouse em- bryonic tissue}

We determined the expression of Epb4.115 in comparison to Crumbs genes and $M p p 5$ on mouse whole mount embryos as well as on isolated organs in different developmental stages by in situ hybridization (Fig. 1). A previous report showed Crb1 expression in mouse embryos as early as E9.5 in the neural tube and retina ${ }^{36}$. In E10.5 embryos, strong Epb4.115 expression is also detected in the developing neural tube and optic vesicle, as well as in the branchial arches and kidney (Fig. 1A). Interestingly, all these tissues also express $M p p 5$ and at least one of the other Crumbs genes, Crb2 or Crb3 (Fig. 1B-D). Two days later, at E12.5, Epb4.115 transcripts were detected in the optic vesicle mainly in the retinal layer, while only a weak signal comparable to the background level was noted in the RPE. This transcript distribution corresponds to the ocular expression pattern of both Mpp5 and Crb1 (Fig. 1E-H). Others have shown expression of $\mathrm{Cr} b 2$ in the adult mouse retina in all cell layers (van den Hurk et al., 2005). Largely overlapping expression patterns for Epb4.115, Mpp5 and Crb2 were found in the developing neural tube. At E11.5, all three genes were expressed along the entire cranial-caudal length of the developing neural tube including the anterior forebrain and the posterior spinal cord (Fig. 1I-N). It is of note that their expression domains were always restricted to the ventricular layer, where proliferative cells are confined. Conversely, Epb4.115, Mpp5 and Crb2 expression was never detected in postmitotic neural compartments at all the stages analyzed. Other organs showed expression of these genes, including the lung and kidney. In the developing lung, Epb4.115, Crb3 and Mpp5, but not Crb2 expression is found in the internal endodermal layer and, in particular, Epb4.115 and Crb3 in the nascent bronchial tips and $M p p 5$ in the forming saccules (Fig. 1O-R). Epb4.115 and Crb3 were found activated in the mesonephric tubules in the 
developing kidney (Fig. 1S-U). Tissue expression of Epb4.115, Mpp5, Crb1, Crb2 and Crb3 is summarized in table S2 (supplementary data). The expression data indicate that Epb4.115, Mpp5 and Crb genes show a close and overlapping expression domain in several tissues containing polarized cells at different time points during embryonic development.

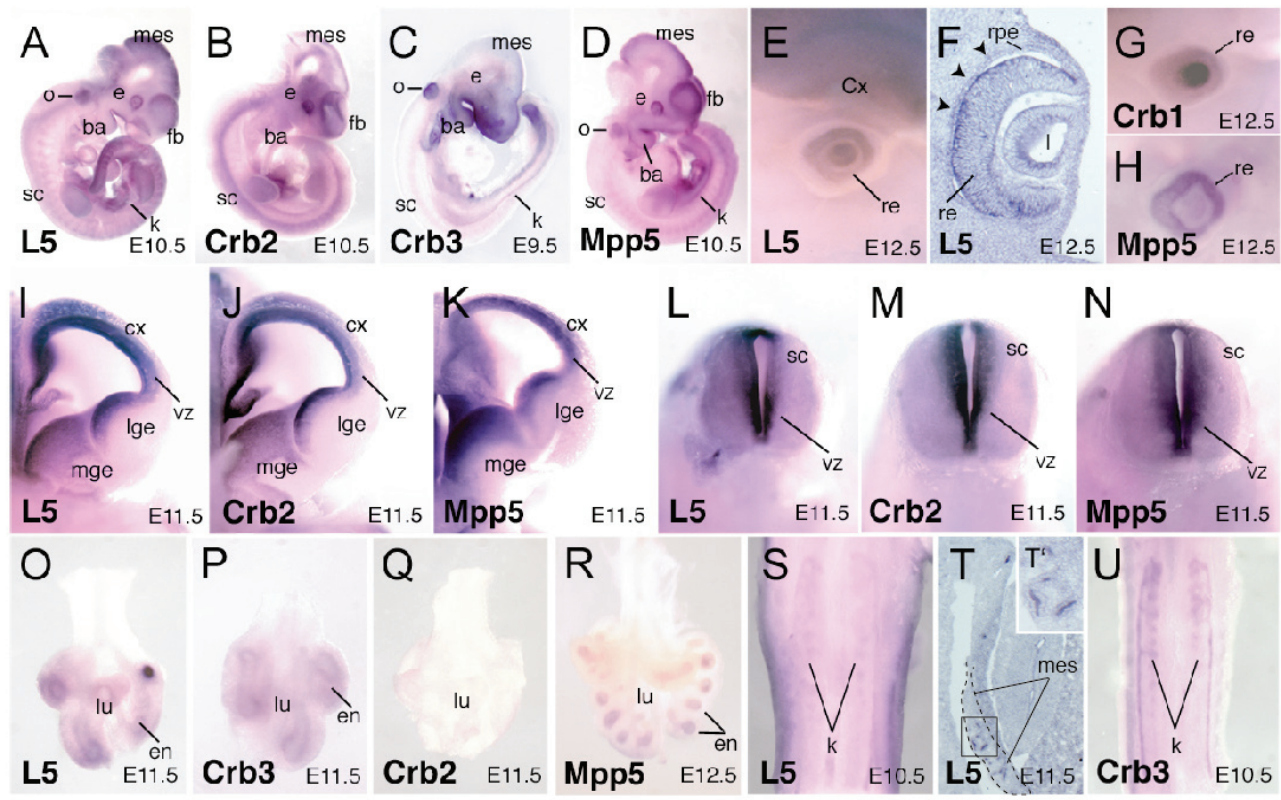

Fig. 1 Crb1, Crb2, Crb3, Mpp5 and Epb4.115 (L5) RNA localization in mouse embryos at different developmental stages. (A-D). Expression pattern of Epb4.115 (A), Crb2 (B), Crb3 (C) and Mpp5 (D) in E9.5-E10.5 whole embryos. Epb41L5 and $M p p 5$ are co-expressed in different tissues like the forebrain (fb), mesencephalon (mes), spinal cord (sc), eye (e), branchial arches (ba), otic vesicle (o) and kidney (k). In all these tissues, either $\mathrm{Crb2}$ (B) or Crb3 (C) or both are expressed. (E-H). Expression in the developing eye of Epb4.115 (E-F), Crb1 (G), Mpp5 (H) is co-localizing in the retina. Epb4.115 transcripts are not detected in the retinal pigmented epithelium (rpe) (arrowheads in F). Expression of Epb4.115, Crb2 and $M p p 5$ in the developing forebrain (I-K) and spinal cord (L-N) in E11.5 dissected mouse neural tube. (O-R) Note Epb4.115 (O), Crb3 (P), Crb2 (Q), Mpp5 (R) expression in developing lungs (lu) isolated from E10.5 and E11.5 mouse embryos. All genes, except $\mathrm{Crb2}$, are expressed in the endodermal layer (en) of the forming bronchioles (Epb4.115, Crb3) or saccules (Mpp5). Both Epb4.115 (S-T), and $\mathrm{Crb3}(\mathrm{U})$ expression is detected in the mesonephric tubules of the developing kidney (k) in E10.5-E11.5 mouse embryos. Dashed lines in T delineate the mesonephric tissue. ( $\left.T^{\prime}\right)$ High magnification of the boxed-area in $(T)$ showing Ep64.115 expression in the endothelial cells of the mesonepric tubules. Cx, cerebral cortex; lge, lateral ganglionic eminence; mge, medial ganglionic eminence; $\mathrm{vz}$, ventricular zone. 


\section{Epb4.115 localizes basolaterally during early development}

To address how Epb4.115 is functionally related to the Crumbs complex, we raised an antibody against the C-terminal part of human EPB41L5 and analyzed the subcellular distribution of these proteins in embryonic tissues. The antibody was found to cross-react with bovine, mouse and rat, and was specific for Epb4.115 on Western blot and immunohistochemistry (supplementary fig. 1). At embryonic stage E15.5, Epb4.115 was associated with the plasma membrane and localized basolaterally in the brain (Fig. 2A), spinal cord (Fig. 2B), kidney and testis (Fig. 2C), intestine (Fig. 2D), all muscles (Fig. 2E) and skin (Fig. 2F). Co-localization studies with Epb4.115 showed overlapping basolateral staining with $\beta$-catenin in epithelial kidney cells (Fig. 2G-I), but not with Crb2, which localized to the apical side (Fig. 2J), and ZO-1, a marker for tight junctions in epithelial cells (Fig. 2K). Likewise, in developing cerebral cortex, Epb4.115 was detected in the basolateral membranes of the proliferating neuroblasts (radial glia cells) as shown by co-localization with the basolateral protein E-cadherin, and the absence of Crb2 staining (a marker of apical cell membranes) (Fig. 2L, M). Thus, in different embryonic epithelial tissues Epb4.115 was present in basolateral positions, adjacent to Crb positive apical domains.

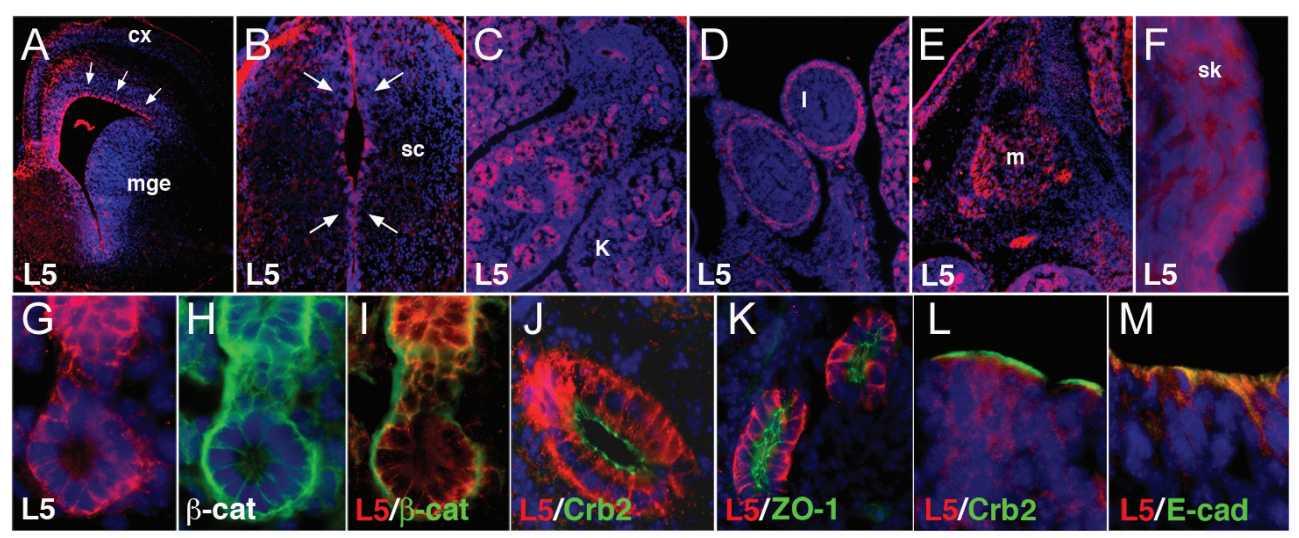

Fig. 2 Epb4.115 protein distribution in embryonic tissues and cellular localization. Cross sections of E15.5 cerebral cortex (A) and spinal cord (B) where Epb4.115 was found to be highly expressed. Strong staining is detected in the luminal side of the entire neural tube fading moving from the ventricle to the peripheral parenchyma (arrows in A, B). Epb4.115 was found to localize in E15.5 embryonic kidney (C), intestinal guts (D), majority of the developing muscles (E) and deep layers of the overlaying immature skin (F). (G-K) Close 
view of Epb4.115 localization in kidney epithelial tubules. (G-I) Epb4.115 and $\beta$-catenin double staining on a renal tubule cross-section. Both stainings appeared largely overlapping on the basolateral cell membrane domains (I). (J, $\mathrm{K})$ Double staining of Epb4.115 with proteins of the cell membrane apical domain such as Crb2 on sections of renal tubules $(\mathrm{J})$ and ZO-1 $(\mathrm{K})$ revealed a close association of both membrane domains without any notable overlap. (L, M) Close view of the cerebral cortex proliferative layer overlying the ventricle. Cortical proliferative cells are forming a pseudo-epithelial structure tightened by intercellular junctions. Epb4.115 staining is strongly detected in a basolateral cellular domain overlapping with E-cadherin (M), but excluded from the most cortical apical side lining the ventricle and stained positive for $\mathrm{Crb} 2(\mathrm{~L})$.

\section{Epb4.115 co-localizes apically with CRB proteins and MPP5 in adult retina and kidney}

In the adult mouse kidney, anti-Epb4.115 stained the basolateral and apical side of the tubule (Fig. 3B). Crb3 staining was confined to the apical domain where it co-localizes with Epb4.115 (Fig. 3A, C).

In adult rat retina, Epb4.115 was found to colocalize with ZA marker $\beta$-catenin (Fig. 3D-F), and also showed substantial overlap with Crb1 (Fig. 3G-I) and Mpp5 stainings (Fig. 3J-L). Besides expression at the region of the OLM, Epb4.115 was found in the RPE, outer nuclear layer (ONL) and outer plexiform layer (OPL). A dotted staining in the inner segments was also observed. On Western blot, the Epb4.115 antibody recognized three different bands at approximately 100, 72 and $45 \mathrm{kD}$ in rat, mouse and bovine retinas (supplementary fig. 1). The $100 \mathrm{kD}$ Epb4.115 protein most likely corresponds to the (mouse) isoform of 731 amino acids (Acc number: AK044959, predicted molecular weight 83 kD). We speculate that posttranslational modifications such as phosphorylation, as described for the Drosophila Yurt protein, could explain the observed difference in migration characteristics ${ }^{25}$. The $72 \mathrm{kD}$ form is not described in the databases, but could represent an unknown alternative isoform due to e.g. splicing variation or posttranslational modification. This form should contain the C-terminal domain of the protein against which our polyclonal antibody was raised. Alternatively but less likely, it may also represent a partially stable degradation product. The smaller band of 45 $\mathrm{kD}$ was found to cross-react with some polyclonal antibodies to GST, since this signal could be completely blocked by adding recombinant GST (supplementary fig. 1D-E). 

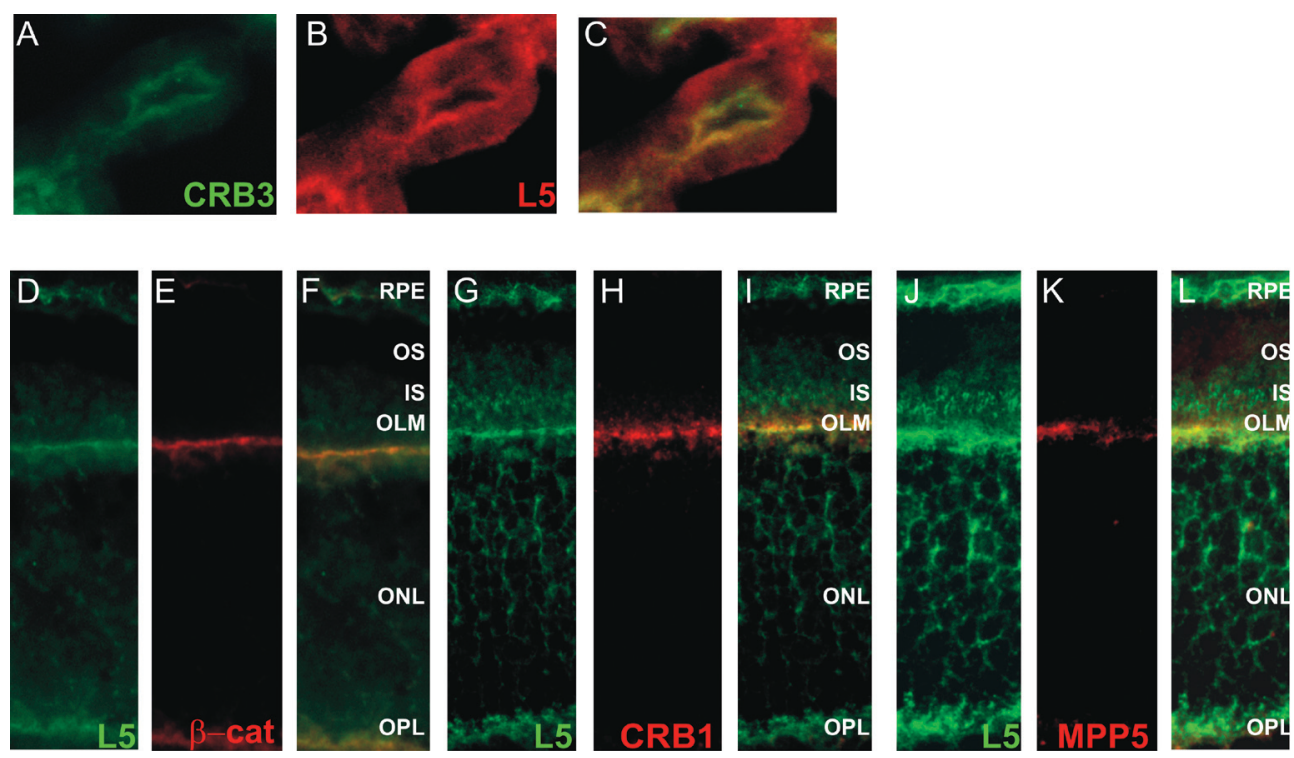

Fig. 3 Co-localization of Epb4.1L5 with CRBs and Mpp5 in adult tissues. (A). Crb3 (green) is found only at the apical side of the kidney tubule, while (B) Epb4.115 (L5, red) is found at the basal and the apical side in adult kidney. (C). Overlap in staining is found at the apical side. (D, G, J) In the retina, Epb4.115 (L5, green) is found in the retinal pigment epithelium (RPE), outer plexiform layer (OPL), outer nuclear layer (ONL), inner segments (IS) and outer limiting membrane (OLM). (D-F) Co-localization with $\beta$-catenin (red), a marker for adherens junctions is found at the OLM. (G-I) Also co-localization of Epb4.1L5 (green) with CRB1 (red) and (J-L) with MPP5 (red) is found at the OLM.

\section{Comparison of FERM domain containing proteins}

An alignment of the intracellular domains of CRB1, CRB2 and CRB3 shows that they all contain a conserved FERM binding motif (GTY) and the well characterized PDZ binding domain (ERLI) that interacts with MPP5 (Fig. 4A). There are several FERM domain containing proteins that hypothetically could bind to the FERM-binding motif.

An alignment of EPB41L5 and its closest homologue EPB41L4B (EHM2), as well as the prototype of the 4.1 protein family (EPB41) and MOESIN shows the similarity of the conserved FERM domain (Fig. 4B). The FERM domain of EPB41L5 is most similar to that of EPB41L4B, while it is less conserved in EPB41 and MOESIN. The FERM domain is composed of three structural modules ( $\mathrm{A}, \mathrm{B}$ and $\mathrm{C}$ ) based on the crystal structure of MOESIN ${ }^{37}$. A potential (spectrin) actin-binding domain ( $\mathrm{SAB}$ or $\mathrm{AB}$ ) that is found in some FERM domain proteins was not identified in EPB41L5 and EPB41L4B (Fig. 4B) ${ }^{38,39}$. 
A
H.s. CRB1 $1^{\text {intra }}$
SNKRATQGTYSPSRQEKEGSRVEMWNLMPPPAMERLI
H.S. CRB2 $2^{\text {intra }}$
RKRRQSEGTYSPSQQEVAGARLEMDSVLKVP PEERLI
H.s. CRB3 ${ }^{\text {intra }}$
REKRQTEGTYRPSSEEQVGARVPPTPNLKLPPEERLI
FERM binding
PDZ binding

B

H.s. EPB41L5

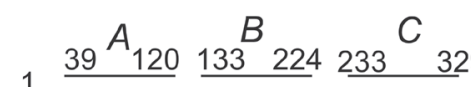

M.m. Epb4115

FERM

733

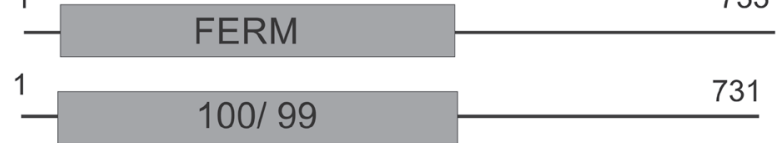

H.s. EPB41L4B

1

913

H.S. EPB41

H.S. MOESIN

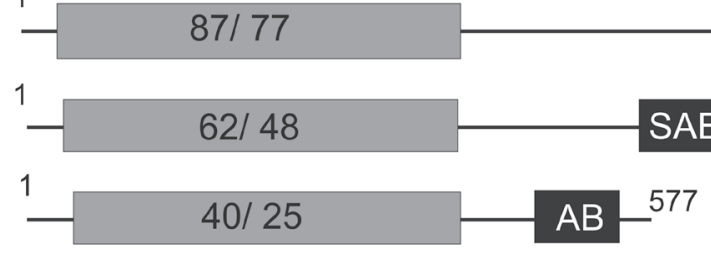

Fig. 4 Sequence conservation in CRB1, CRB2 and CRB3 and alignment of FERM domain proteins. (A). The 37-amino acid intracellular domains of CRB1, CRB2 and CRB3 are aligned. The conserved FERM domain binding site (GTY_E) and an ERLI motif are underlined. Constructs with deletion of part of the FERM domain binding site $(\triangle \mathrm{GTY})$ or the terminal four amino acids $(\triangle \mathrm{ERLI})$ were made. (B). Human (H.s.) EPB41L4B, human EPB41, mouse (M.m.) Epb4.115 and human MOESIN were individually aligned with human EPB41L5. The percentages of similar and identical amino acid sequence of the conserved FERM domain are shown. A FERM domain consists of three structural modules (A, B and C). EPB41 contains a conserved spectrin-actin binding domain (SAB) that is not found in EPB41L5. The terminal 140 amino acids of MOESIN contain an actin-binding (AB) domain.

\section{Interaction studies using yeast two-hybrid systems}

We analyzed EPB41L5, EPB41L4B, EPB41 and MOESIN for proteinprotein interaction with CRBs using two different yeast two-hybrid systems. In the GAL4 based system, the FERM domain of EPB41L5, EPB41L4B and MOESIN, as well as full length EPB41L5 were found to interact with the intracellular domain of CRB1, CRB2 and CRB3 (Fig. 5A), whereas full length EPB41 did not. The yeast reporter assays indicate that EPB41L5 has the highest binding affinity for the intracellular domain of CRB proteins. The same results were observed when $\mathrm{pBD}$ and pAD tags were swapped (data not shown). An EPB41L5 construct with 
an intact FERM domain bound strongly to CRB1 ${ }^{\text {INTRA }}$. Removal of the first structural module of the FERM domain (EPB41L5 ${ }^{\text {FERM_BC }}$ ) abrogated binding to CRB1 (Fig. 5A).

\begin{tabular}{|c|c|c|c|}
\hline \\
\hline pBD & pAD & Growth & Coloring \\
\hline$\overline{\mathrm{CRB}} 1^{\text {INTRA }}$ & EPB41L4B ${ }^{\text {FERM }}$ & + & $+/-$ \\
\hline $\mathrm{CRB} 1^{\text {INTRA }}$ & MOESIN ${ }^{\text {FERM }}$ & + & - \\
\hline CRB $1^{\text {INTRA }}$ & EPB41 $1^{\mathrm{FL}}$ & . & $=$ \\
\hline CRB $1^{\text {INTRA }}$ & EPB41L5 $5^{\text {FERM_ABC }}$ & ++ & + \\
\hline CRB $1^{\text {INTRA }}$ & EPB41L5 $5^{\text {FERM_BC }}$ & - & - \\
\hline CRB $1^{\text {INTRA }}$ & EPB41L5 ${ }^{\mathrm{FL}}$ & ++ & + \\
\hline$\overline{\mathrm{CRB}} 1^{\text {INTRA }}$ & $\mathrm{MPP}^{\mathrm{PDZ}}$ & ++ & + \\
\hline
\end{tabular}

B

\begin{tabular}{ll|cc} 
pSos & pMyr & Glu 37 & Galac 37 \\
\hline CRB1 ${ }^{\text {INTRA }}$ EPB41L5 & FERM_ABC & - & + \\
CRB1 $^{\text {INTRA }}$ EPB41L5 & FERM_BC & - & - \\
CRB1 $^{\text {INTRA }}$ EPB41L5 & - & - \\
\hline CRB1 $^{\text {INTRA }}$ MPP5 $^{\text {PDZ }}$ & - & +
\end{tabular}

C
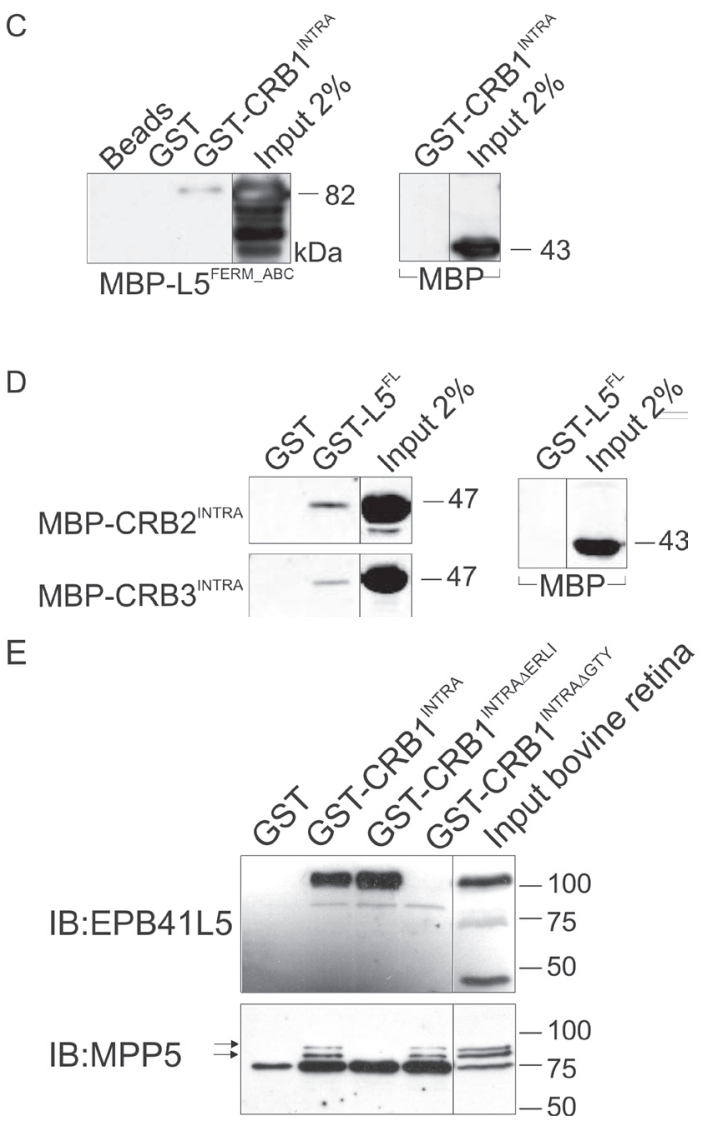
Fig. 5 Identification of interaction between CRB1 and FERM domain protein EPB41L5. (A) CRB1 ${ }^{\mathrm{INTRA}}$ fused to $\mathrm{pBD}$ interacted with the FERM domain of EPB41L5, EPB41L4B and MOESIN, but not with EPB41 fused to pAD. These interactions were determined by growth on selection plates and coloring in an $\alpha$-galactosidase plate-assay. The strongest interaction was found between EPB41L5 and CRB1. The known interaction between the intracellular domain of CRB1 and the PDZ domain of MPP5 is used as a positive control. The FERM domain of EPB41L5 (EPB41L5 $5^{\mathrm{FERM} \_\mathrm{ABC}}$ ) binds as strong as the full length construct $\left(\mathrm{EPB} 41 \mathrm{~L}^{\mathrm{FL}}\right)$ to the intracellular domain of CRB1. No interaction was found between CRB1 and EPB41L5 with a truncated FERM domain (EPB41L5 $\left.{ }^{\text {FERM_BC }}\right)$. (B) In the Cytotrap system, no growth of yeast is seen on control glucose plates at $37^{\circ} \mathrm{C}$, as expected. On galactose plates at $37^{\circ} \mathrm{C}$ (galac 37), pMyr-EPB41L5 and pMyr-MPP5 proteins are expressed and targeted to the cell membrane. pSos-CRB1 ${ }^{\text {INTRA }}$ interacts with pMyr-EPB41L5 $5^{\text {FERM_ABC }}$ and $\mathrm{pMyr}-\mathrm{MPP} 5^{\mathrm{PDZ}}$, resulting in yeast growth. CRB1 does not interact with the full length EPB41L5, nor with EPB41L5 having a truncated FERM domain. (C) In a GST-pull down assay, CRB1 ${ }^{\text {INTRA }}$ interacted with the FERM domain of EPB41L5. GST protein alone and only beads were used as a negative control. In addition, non-specific binding of the his-MBP tag to the GST-CRB1 fusion protein is not found in the control. (D) Full length EPB41L5 interacts with CRB2 $2^{\text {INTRA }}$ as well as CRB3 ${ }^{\text {INTRA }}$. (E) GST-pull down from retinal lysates using the intracellular domain of CRB1, either without the C-terminal PDZ domain binding motif (GST-CRB1 ${ }^{\text {INTRA } E \text { ERLI }}$ ) or without the FERM binding motif (GST-CRB1 ${ }^{\text {INTRA } \triangle G T Y}$ ). EPB41L5 binds specifically to GST-CRB1 ${ }^{\text {INTRA }}$ and not to GST. When the GTY motif is deleted, binding is lost. MPP5 in bovine retina is detected as a doublet with bands at 78 and $80 \mathrm{kD}$. At $75 \mathrm{kD}$, a background band is detected. MPP5 binds specifically to CRB1 $1^{\text {INTRA }}$ and CRB1 ${ }^{\text {INTRAAGTY, }}$ but neither to GST or to CRB1 $1^{\text {INTRA }}$ ERLI.

The binding of EPB41L5 to CRB1 was confirmed in the SOS recruitment system. This system is based on the activation of the Ras signal transduction cascade upon membrane targeting of SOS (fused to CRB1 ${ }^{\text {INTRA }}$ ) due to interaction with EPB41L5. Although in this system we failed to detect association of the full length EPB41L5 protein to CRB1, we confirmed interaction with its FERM domain, and not with its truncated FERM domain as expected (Fig. 5B).

\section{The FERM domain of EPB41L5 binds to CRB proteins}

The intracellular domain of CRB1 fused to GST (GST-CRB1 ${ }^{\text {intra }}$ ) efficiently pulled down the his-MBP tagged FERM domain of EPB41L5 (Fig. 5C). This binding is specific, since neither GST nor unloaded beads could pull down EPB41L5 protein. In accordance with the yeast data, 
binding was abrogated when using the truncated FERM domain (Fig. $\mathrm{S} 2 \mathrm{~A}$ ). As the juxtamembrane FERM binding motif is conserved in all $\mathrm{CRB}$ proteins, it was not surprising that EPB41L5 interacts with the intracellular domain of CRB2 and CRB3 (Fig. 5D).

Binding of EPB41L5 to CRB proteins depends on the GTY amino acid sequence, since removal of this motif in a GST-fusion construct of the intracellular domain of CRB1 resulted in loss of binding. The ERLI motif was not required for interaction between EPB41L5 and CRB proteins (Fig. S2B).

In addition, full length Crb3 and Epb4.115 form a complex in MDCK cells. Immunoprecipitation of stably transfected p75-Crb3 from these cells efficiently co-precipitated Myc-Epb4.115 from MDCK cells as determined with a myc-antibody on Western blot. When myc-Epb4.115 was immunoprecipitated with a myc antibody, p75-Crb3 co-precipitated (Fig. S2C).

In the retina, both EPB41L5 and MPP5 bind to the intracellular domain of CRB1 (Fig. 5E). A GST fusion protein containing the intracellular part of CRB1 pulled down MPP5 as well as EPB41L5. When the GTY motif was removed, EPB41L5 was no longer able to bind to CRB1, while binding of MPP5 to CRB1 was not affected. Only when the Cterminal ERLI motif was removed, binding of MPP5 was lost, whereas binding of EPB41L5 was not. This experiment confirmed the binding of EPB41L5 and MPP5 to the two conserved binding motifs in the intracellular domain of CRB1.

\section{EPB41L5 interacts directly with CRB binding partner MPP5}

MPP5 contains a HOOK domain with a potential interaction site for FERM domain proteins. Interaction was found between the FERM domain of EPB41L5 and the HOOK domain of MPP5 or full length MPP5 in the yeast two-hybrid system (Fig. 6A). The affinity of the HOOK domain for the FERM domain of EPB41L5 was confirmed in a GST pull down assay (Fig. 6B). In HEK293 cells, the full length proteins formed a complex (Fig. 6C). The EPB41L5 antibody attached to beads pulled down EPB41L5 and MPP5, whereas non-specific guinea pig IgG's did not. Thus, in addition to binding the FERM binding motif of CRB proteins, EPB41L5 interacts directly with the HOOK domain of MPP5. 
A

\begin{tabular}{ll|cc} 
pAD & pBD & Growth & Alfa-gal \\
\hline EPB41L5 $^{\text {FERM }}$ & MPP5 $^{\text {FL }}$ & ++ & $+/-$ \\
EPB41L5 $^{\text {LL }}$ & MPP5 $^{\text {FL }}$ & - & - \\
\hline MPP5 $^{\text {HOOK }}$ & EPB41L5 $^{\text {FERM }}$ & + & - \\
MPP5 $^{\text {SH3+HOOK }}$ & EPB41L5 $^{\text {FERM }}$ & + & -
\end{tabular}

B

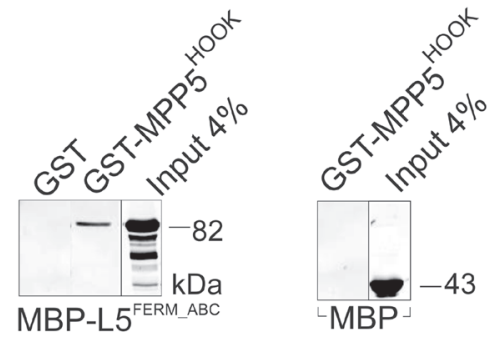

C

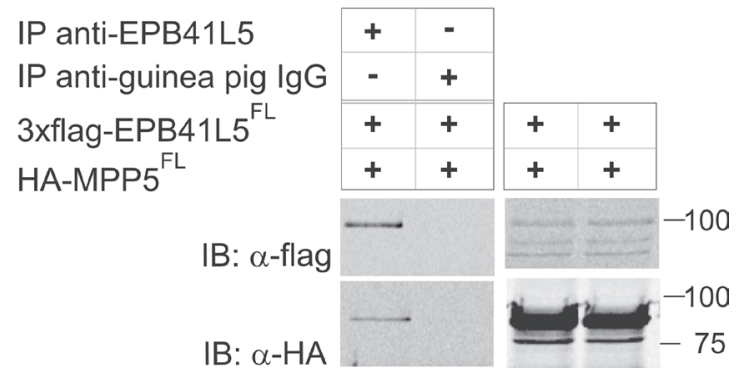

Fig. 6 Direct interaction between MPP5 and EPB41L5. (A). The FERM domain of EPB41L5 binds to full length MPP5. The domain of MPP5 responsible for binding to EPB41L5 is restricted to the HOOK domain using constructs with the SH3+HOOK domain or only the HOOK domain. (B). GST-pull down confirms that the HOOK domain of MPP5 binds to the intact FERM domain of EPB41L5. (C). Anti-EPB41L5 antibody co-immunoprecipitates EPB41L5 with full length MPP5 from HEK293 cells. In the control lane, non-specific guinea pig $\operatorname{IgG}$ coupled to prot A/G agarose beads did not co-immunoprecipitate EPB41L5 and MPP5.

\section{Epb4.115 overexpression affects tight junction formation in MCDK cells}

The dynamic localization of Epb4.115 on cell membranes of epithelial like-tissues such as kidney and neural structures and its interaction with the CRB-MPP5 protein complex prompted us to test the involvement of Epb4.115 in establishing cell polarity and cell junction formation. 
We established MDCK cell lines overexpressing a myc-tagged Epb4.115 construct. Clones 1 and 2 expressing myc-Epb4.115 at different levels, as tested by Western-blot (Fig. 7A), were selected for further analysis. Control and myc-Epb4.115 overexpressing MDCK cells were subjected to a "calcium switch" protocol in order to follow cell re-polarization and establishment of cell junctions. To check for correct cell tightness and cell junction assembly, control and myc-Epb4.115 expressing cell monolayers were subjected to $\left[{ }^{3} \mathrm{H}\right]$ inulin leakage test (Fig. 7B). Briefly, cell monolayers in filter chambers were treated with $\left[{ }^{3} \mathrm{H}\right]$ inulin added in the apical chamber. The amount of $\left[{ }^{3} \mathrm{H}\right]$ inulin found in the basal chamber provide hints about the leakage of the cell monolayer. After 1 hour of $\left[{ }^{3} \mathrm{H}\right]$ inulin supplementation, control cell monolayers were tight enough to allow the crossing of only $1.5 \%$ of total radioactivity. On the contrary, both myc-Epb4.115 overexpressing cell monolayers partially failed to block $\left[{ }^{3} \mathrm{H}\right]$ inulin leakage. Interestingly, leakage defects were stronger in the higher myc-Epb4.115 expressing cells (clone 2), indicating a specific effect of the transgene in affecting or delaying cell junction assembly. To look for the cellular impairment responsible for these defects, cells were analyzed for cell junction formation and polarity (Fig. 7C). While in control MDCK cells polarization was completely restored after 12 hours, myc-Epb4.115 overexpressing cells (clone 2) displayed disorganized cell contacts at the same time point. Interestingly, ZO-1 and PATJ stainings revealed disorganization and repression of tight junction formation in Epb4.115 overexpressing cells with respect to control cells. Both proteins were either completely lost in some junctions or strongly repressed, indicating a failure in tight junction formation (Fig. 7 C-H). On the contrary, $\beta$-catenin expression was not strongly altered indicating rather normal adherens junction structures (data not shown). Thus, Epb4.115 overexpression specifically represses tight junction formation leading to cell-cell contact disorganization. Later analysis, 24 hours after switch to normal growth medium, showed a strong recovery of cellular junction in Epb4.115 overexpressing cells, although occasionally loss or reduction of ZO-1 and PATJ staining at cell borders was still detected (data not shown). All together, these results indicate that during epithelial cell polarization, Epb4.115 is a negative regulator of tight junction assembly that may contribute to the correct positioning of these structures in the cells. 

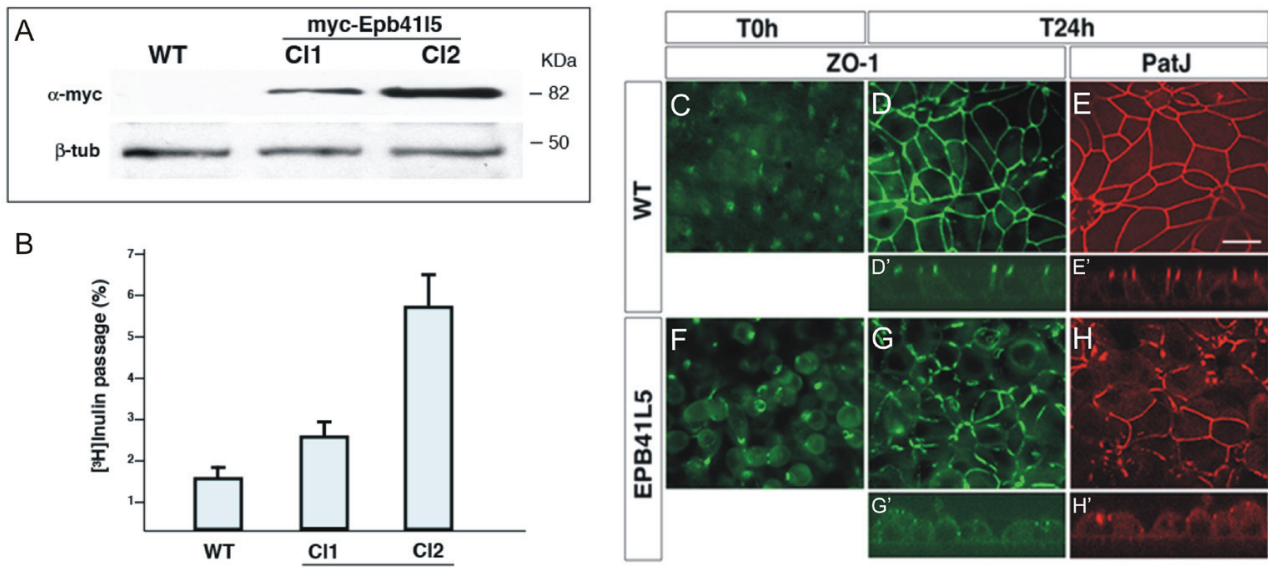

Fig. 7 Overexpression of Epb4.11L5 affects tight junction formation in calcium-switch dependent MDCK cell re-polarization. (A). Western-blot with an anti-myc antibody showing stable MDCK cell lines expressing low (clone 1) and high (clone 2) levels of the myc-Epb4.115 transgene. (B). Analysis of cell monolayer tightness by $\left.{ }^{3} \mathrm{H}\right]$ inulin leakage assay, 12 hours after calcium switch. Clones 1 and 2 expressing myc-Epb4.115 show about 1.6 - 4 time fold leakage of $\left[{ }^{3} \mathrm{H}\right]$ inulin compared to control cells in a transwell chamber, respectively. (C-H) Analysis of tight junction assembly in control (C-E) and clone 2 overexpressing myc-Epb4.115 (F-H) cells in a "calcium-switch" assay. $(\mathrm{C}, \mathrm{F})$ Complete loss of polarity and junction structures in cells treated with a medium lacking $\mathrm{Ca}^{2+}(\mathrm{T}=0)$ is seen. Tight junctions are completely restored in control cells from a change in high $\mathrm{Ca}^{2+}$ culture medium after 12 hours, as visualized with ZO-1 and PATJ protein localization (D, D', E, E'). Conversely, clone 2 expressing myc-Epb4.115 cells display a severe cellular disorganization and impairment in tight junction assembly as shown by loss of ZO-1 and PATJ proteins in cell borders (arrows in $G, G^{\prime}, H, H^{\prime}$ ). D', E', G', H', Z-sections showing the apical-basolateral localization of the proteins. Bar, $10 \mu \mathrm{m}$.

\section{DISCUSSION}

In this study, we show that FERM domain protein Epb4.115 is associated with the mammalian CRB-MPP5 complex. We found that during development, Epb4.115 is co-expressed with at least one Crumbs gene and $M p p 5$ in different mouse epithelial tissues. For example, Crb1, Crb2, Epb4.115 and $M p p 5$ are co-expressed in the neural tube, while $C r b 3, M p p 5$ and Epb4.115 are co-expressed in the kidney and lung. In addition, Crb1, $M p p 5$ and Epb4.115 are co-expressed in the retina. Early gene expression 
of Epb4.115 in the developing neural tube indicates an important developmental function. Indeed, mouse mutant embryos (lulu), which have a null allele for Epb4.115, are impaired in neural tube closure and arrest at E8.5 with defects in mesoderm, endoderm and neural plate morphogenesis 40,41 .

Epb4.115 localizes to the basolateral side of epithelial cells in several embryonic mouse tissues at E15.5. In adult tissues, Epb4.115 was found at the apical side of epithelia. During development Epb4.115 is recruited to the apical domain, demonstrating an important regulation of its expression during development. Similarly, in Drosophila embryonic epithelia that express Crumbs, as well as in developing photoreceptors, the Epb4.115 homologue Yurt is associated with the basolateral membrane. Starting from stage 13, Yurt was detected apical to the ZA in epithelia, a region that corresponds to the vertebrate tight junction. In the eye, at $90 \%$ of pupal development and in adult flies, Yurt was detected at the stalk membrane, the apical side of photoreceptors ${ }^{25}$.

In the mammalian retina, Epb4.115 is present at the OLM and also showed substantial co-localization with Crb1 and Mpp5 apical to the OLM. The function of Crumbs at the OLM is to maintain adherens junction between photoreceptors and Müller glia cells, while Mpp5 was recently implicated in correct targeting of Crb1 to this region in Müller glia cells ${ }^{13,14,42}$. Recently, it was demonstrated that zebrafish nok (Mpp5) is essential for the establishment and/ or maintenance of the OLM in the retinal neuroepithelium during early development ${ }^{17}$. Adherens junctions in the retinal neuroepithelium might be precursors for the junctions later found at the OLM in the fully developed retina. The presence of the Crumbs, Epb4.115 and Mpp5 gene products in the mammalian retinal neuroepithelium as well as in the fully developed retina suggest an important function in regulating polarity.

Interaction studies revealed that an intact FERM domain of Epb4.115 could strongly bind to the conserved GTY motif found in the intracellular domains of all three Crumbs homologues. A new aspect in the composition of the mammalian CRB complex is the direct association of Epb4.115 with MPP5. Remarkably, this binding also involves the FERM domain of Epb4.115 and the lysine rich HOOK domain of MPP5. These binding studies as well as co-expression and co-localization in several tissues suggest that a CRB-Mpp5-Epb4.115 protein complex could be active in different tissues, such as the kidney and the retina, exerting an important function in cell polarity. Interestingly, conservation of this 
complex is observed between species ${ }^{19,25,26}$. The Drosophila FERM protein Yurt is able to bind Crumbs via the GTY motif. A Crumbs-MPP5EPB41L5 complex was detected in zebrafish, and was shown to negatively regulate photoreceptor apical size.

We have shown that in the retina, the intracellular domain of Crb1 can bind to Epb4.115 and MPP5. A potential stabilization of the CRB3MPP5 complex by a FERM domain protein was suggested by others ${ }^{2}$. However, mutating the FERM binding motif of CRB1 did not reduce the binding of MPP5, nor did a truncated PDZ binding motif decrease binding to the FERM protein. We did also not observe that Epb4.115 promotes binding of Mpp5 to Crumbs in the absence of the ERLI motif of the latter. It is possible that binding of Epb4.115 to Mpp5 is too weak to be detected in this assay or that the affinity of Mpp5 to the FERM protein is not high enough to recruit Mpp5 into the complex when the ERLI sequence is removed. The same holds true for the inability of Mpp5 to recruit Epb4.115 when the FERM binding motif of CRB1 was removed. We showed that the full length MPP5 and EPB41L5 proteins, as well as the interacting domains bind to each other. Also in zebrafish, the EPB41L5/YMO1-MPP5 interaction is conserved, since the FERM domain of YMO1 pulls down Nok from retinal lysates ${ }^{26}$. It might be that the interaction between Epb4.115 and Mpp5 is regulated by other proteins or signaling events, or only occur at specific time points during complex formation.

Epb4.115 binding to CRBs and Mpp5 could be simultaneous or mutually exclusive, since the FERM domain of Epb4.115 is involved in both protein-protein interactions. It is known that both MAGUK and 4.1 proteins form ternary complexes at the plasma membrane ${ }^{43}$. Transmembrane protein Glycophorin C forms a complex with MAGUK protein MPP1 and EPB41 at the plasma membrane of red blood cells ${ }^{44}$, whereas neurexin and syndecan-2 bind to CASK and EPB41 in neurons ${ }^{45,46}$. For EPB41 binding to Glycophorin C and Mpp1, different parts within the FERM domain are used and therefore is not competitive. Future experiments will have to determine if the same holds true for the Epb4.115 binding to MPP5 or CRBs.

In light of the interaction of Epb4.115 with the Crumbs complex and the fact that overexpression of Epb4.115 affects the stabilization of the tight junctions, it is tempting to propose a mechanism in which Epb4.115 might be recruited to the plasma membrane and thereby recruits Crumbs complex partners. Mutations in either the PDZ domain or FERM do- 
main binding motif of CRB3 led to the inability of CRB3 to induce tight junctions (TJs) in a MCF10 cell model, leaving an important function for the presently identified Epb4.115 protein in addition to Mpp5 ${ }^{2}$. Several additional proteins that are present in the macromolecular Crumbs protein complex have been implicated in the formation of TJs in mammalian epithelia. It was shown that binding of PATJ to the L27N domain of Mpp5 is important for its localization ${ }^{34}$. In turn, PATJ binds to TJ-associated proteins $\mathrm{ZO}-3$ and claudin that mediate cell adhesion at this site 47. The L27C domain of Mpp5 binds to Lin7 and the N-terminal part of MPP5 binds directly to the PAR6-PAR3-aPKC polarity complex through direct association with PAR6 ${ }^{23,48}$. Loss of MPP5 and overexpression of Epb4.115 both lead to delayed TJ formation ${ }^{49}$. When overexpressed, Epb4.115 might influence TJ formation by regulating MPP5's availability for other interaction partners through its direct interaction with the HOOK domain of MPP5. With less MPP5 present to perform a central scaffolding function, TJs could be formed less efficient. The number of different proteins that are involved in tight junction formation illustrates the complexity of the interplay between polarity molecules.

We can conclude that EPB41L5 binds to conserved motifs of Crumbs proteins and MPP5 and plays an important role in the mammalian Crumbs protein complex. Our findings provide strong indications that EPB41L5 is not only important in cell polarity processes in the retina, but in many epithelial-derived tissues throughout development. 


\section{ACKNOWLEDGEMENTS}

We thank Theo A. Peters for his technical assistance concerning the immunohistochemistry on retinal tissue. This work was financed in part by grants from Netherlands Organization for Scientific Research (NWO) (912-02-018) to F.P.M.C., European Commission (EC) (QLRT-200002253) to A.L.B., V.B. and F.P.M.C., and European Commission IP 'EVI-GenoRet' (LSHG-CT-2005-512036), to R.R. and F.P.M.C. 


\section{SUPPLEMENTARY MATERIAL}

A
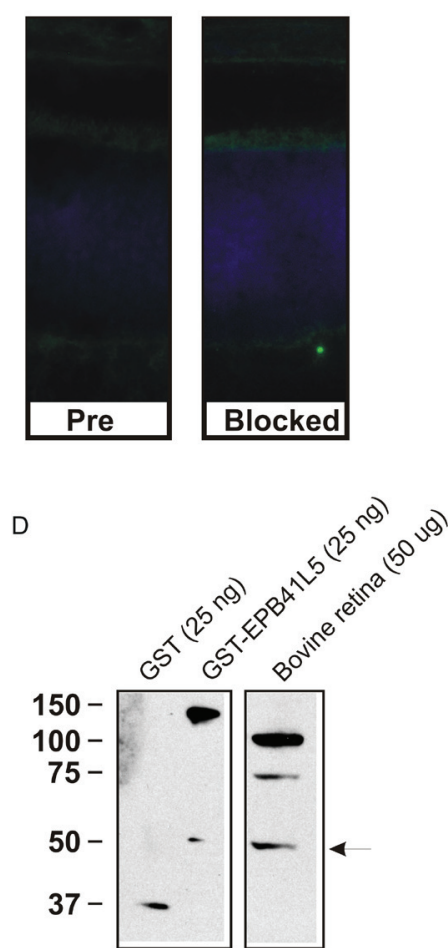

IB: EPB41L5
B

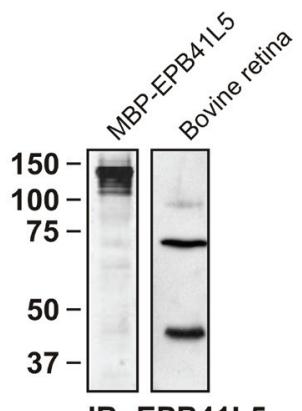

IB: EPB41L5
C

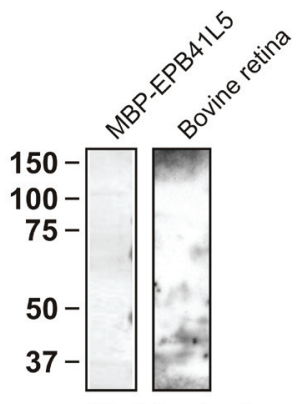

IB: blocked

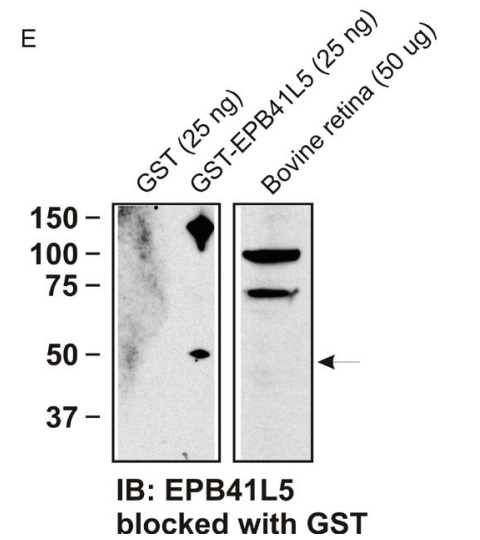

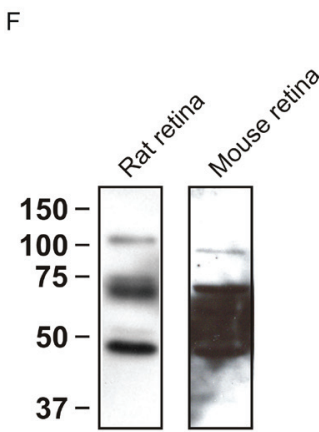

IB: EPB41L5

S1. Characterization of the EPB41L5 antibody. (A). Pre-immune serum (Pre) of the immunized guinea pig does not give any signal in immunohistochemistry on rat retinal slices. In the second panel, the signal of Epb4.115 in rat retina could be completely blocked by incubating the affinity purified EPB41L5 antibody with $500 \mu \mathrm{g}$ of GST-fusion protein prior to incubation with retinal tissue. (B). The affinity purified EPB41L5 antibody recognizes recombinant MBP-tagged as well as two different forms of endogenous EPB41L5 in bovine retina of $100 \mathrm{kD}$ and $72 \mathrm{kD}$ on immunoblot (IB). In addition, a smaller band of $45 \mathrm{kD}$ was detected. (C). The obtained signals in bovine retina with the EPB41L5 antibody could be completely blocked with $500 \mu \mathrm{g}$ GST-EPB41L5 fusion protein. (D). The affinity purified EPB41L5 antibody recognizes recombinant GST-tagged EPB41L5 protein and has some affinity for recombinant GST. Three bands are recognized in bovine retina of $100 \mathrm{kD}, 72 \mathrm{kD}$ and $45 \mathrm{kD}$. (E). The signal of $45 \mathrm{kD}$ in Western blot with the EPB41L5 antibody could be completely blocked with $250 \mu \mathrm{g}$ recombinant GST protein from Schistosoma japonicum. Whether or not this $45 \mathrm{kD}$ band corresponds to endogenous bo- 
vine GST, is not known. The $100 \mathrm{kD}$ and $72 \mathrm{kD}$ bands correspond to different forms of EPB41L5, while the $45 \mathrm{kD}$ band cross-reacts with some antibodies to the recombinant GST-tag that are present in the polyclonal. (F). In rat and mouse retina, three bands of $100 \mathrm{kD}, 72 \mathrm{kD}$ and $45 \mathrm{kD}$ are detected as well.

A
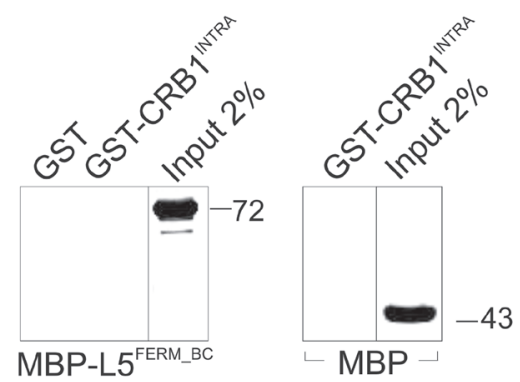

C

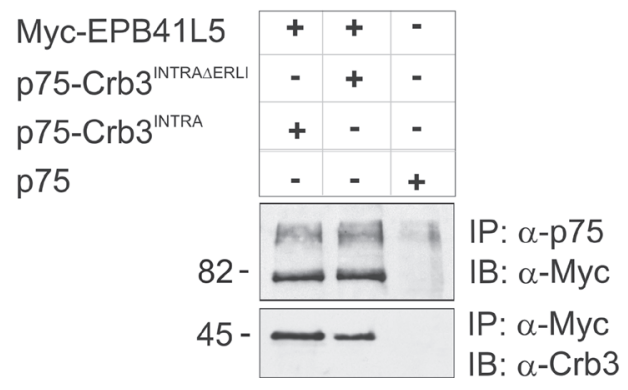

B

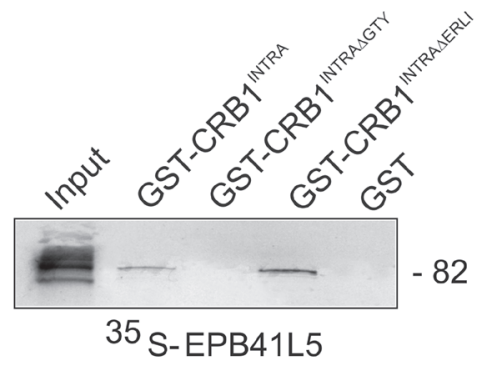

S2. GST-pull down assays. (A). When the truncated FERM domain of EPB41L5 (EPB41L5 ${ }^{\text {FERM_BC }}$ ) was used in a GST pull down assay, the interaction with CRB1 ${ }^{\text {INTRA }}$ was lost. (B). Radioactive-labeled Epb4.115 binds to the intracellular domain of Crb1 with or without the C-terminal ERLI-motif. When the FERM domain binding site is deleted ( $\triangle G T Y)$, the interaction with Epb4.115 is lost. (C). MDCK cells stably expressing p75-CRB33 ${ }^{\text {INTRA }}$ or p75CRB3 ${ }^{\text {INTRA } \triangle E R L I}$ were transfected with Myc-Epb4.115. Lysates were immunoprecipitated (IP) with p75 antibody. Proteins were resolved on SDS-PAGE and immunoblotting using anti-myc antibody. Epb4.115 associates with CRB3 $3^{\text {INTRA }}$ as well as CRB $3^{\text {INTRAAERLI }}$. The reverse experiment was performed using antimyc antibody for IP and anti-CRB3 antibody for detection on immunoblot. P75-CRB3 ${ }^{\text {INTRA }}$ as well as p75-CRB3 ${ }^{\text {INTRA }}$ ERLI were found in the complex when immunoprecipitating myc-Epb4.115. 
Supplementary table 1. Sense and antisense primer sequences for Gateway constructs

\begin{tabular}{|c|c|c|}
\hline Construct (a.a. position) & Sense & Antisense \\
\hline hEPB41L5 $5^{\mathrm{FL}}(1-733)$ & 5'-ATGCTGAGTTTCTTCCG-3' & 5'-TCAGAGCTCAGTGGTCAG-3' \\
\hline hEPB41L5 $5^{\text {FERM_ABC }}(29-353)$ & 5'-GCGCCGCCACACATATTCCT-3' & 5'-GATACTCTGTTTTCCCACTA-3' \\
\hline hEPB41L5 $5^{\text {FERM_BC }}(120-353)$ & 5'-TAGTTAAGTTTTATTCCTCAGAACC-3' & 5'-GATACTCTGTTTTCCCACTA-3', \\
\hline hCRB2 $2^{\text {INTRA }}(1249-1285)$ & 5'-GGATCCTGGCAGCCCGAAAGC-3' & 5'-CCTAGATGAGTCTCTCCTCCG-3' \\
\hline $\mathrm{hCRB} 3^{\text {INTRA }}(84-120)$ & 5'-TGGTGCGGAAGCTTCGGGAGAA-3' & 5'-GCGTTCAGATGAGCCGCTCT-3' \\
\hline hMPP5 $^{\text {НООК }}(408-466)$ & 5'-GAAGAAGCCATGAAACAAACC-3' & 5'-ATAGGTTAAGATCTCCTCGTTG-3' \\
\hline hMPP5 $^{\mathrm{FL}}(1-675)$ & 5'-ATGACAACATCCCATATGAAT-3, & 5'-TCACCTCAGCCAAGTGGATGG-3', \\
\hline hMPP5 $5^{\text {SH3+ноОк }}(337-458)$ & 5'-ACAGTAATCCATGTAAAAGCTC-3' & 5'-ATAATCATCATTTTTATTGG-3' \\
\hline hEPB41L4B FERM_ABC $_{(67-263)}$ & 5'-CCGCCAAGGCCACCCTCTACTG-3' & 5'-GATATTCTGTCCGCCCACTGAACT-3' \\
\hline hEPB41L4B ${ }^{\text {FERM_BC }}(148-263)$ & 5'-TAGTTAAATACTATTCTTCAGAACC-3' & 5'-GATATTCTGTCCGCCCACTGAACT-3' \\
\hline hMOESIN $^{\text {FERM_ABC }}(1-306)$ & 5'-ATGCCCAAAACGATCAGTGTGCG-3' & 5'-CCTTCATCTGCTGCACCTCA -3' \\
\hline hMOESIN $^{\text {FERM_BC }}(82-306)$ & 5'-GTGCCAAGTTCTACCCTGAGG -3' & 5'-CCTTCATCTGCTGCACCTCA -3' \\
\hline $\mathrm{hEPB} 41^{\mathrm{FL}}(1-720)$ & 5'-ATGACAACAGAGAAGAG-3' & 5'-TCATGTGACAAAGTACTCAC-3' \\
\hline hEPB41L5 $5^{\text {c-ter }}(476-733)$ & 5'-ACATCCCAAGCACTGAATG -3' & 5'-TCAGAGCTCAGTGGTCAG-3' \\
\hline hEPB41 ${ }^{\text {C-ter }}(476-720)$ & 5'-TTATGGAAAGTCTGTGTAG-3' & 5'-TCATGTGACAAAGTACTCAC-3' \\
\hline hCRB1 $1^{\text {INTRA } \triangle \mathrm{GTY}}(1370-1406)$ & 5'-AGGGCAACTCAGAGCCCCAGCCGTCA-3, & 5'-CCTAAATCAGTCTCTCCATTGC-3' \\
\hline hCRB1 $1^{\text {INTRA } \triangle E R L I}(1370-1402)$ & 5'-TCACCTCCAACAAAAGGGCAAC-3' & 5'-TTACATTGCAGGGGGTGGCATC-3' \\
\hline
\end{tabular}

Supplementary table 2. Summary of mRNA expression of Crb-complex members in mouse embryonic tissues

\begin{tabular}{c|ccccccc}
\hline & Neural tube & retina & kidney & skin & lung & testis & muscle \\
\hline Crb1 & + & + & - & - & - & - & - \\
Crb2 & + & - & - & - & - & - & - \\
$C r b 3$ & - & - & + & - & + & - & - \\
Epb4.115 & + & + & + & + & + & + & + \\
Mpp5 & + & + & + & - & + & - & - \\
\hline
\end{tabular}




\section{REFERENCES}

1. Richard, M. et al. Towards understanding CRUMBS function in retinal dystrophies. Hum.Mol.Genet. 15 Spec No 2, R235-R243 (2006).

2. Fogg, V. C., Liu, C. J., \& Margolis, B. Multiple regions of Crumbs3 are required for tight junction formation in MCF10A cells. Journal of Cell Science 118, 2859-2869 (2005).

3. Knust, E. \& Bossinger, O. Composition and formation of intercellular junctions in epithelial cells. Science 298, 1955-1959 (2002).

4. Bachmann, A., Schneider, M., Theilenberg, E., Grawe, F., \& Knust, E. Drosophila Stardust is a partner of Crumbs in the control of epithelial cell polarity. Nature 414, 638-643 (2001).

5. Grawe, F., Wodarz, A., Lee, B., Knust, E., \& Skaer, H. The Drosophila genes crumbs and stardust are involved in the biogenesis of adherens junctions. Development 122, 951-959 (1996).

6. Hong, Y., Stronach, B., Perrimon, N., Jan, L. Y., \& Jan, Y. N. Drosophila Stardust interacts with Crumbs to control polarity of epithelia but not neuroblasts. Nature 414, 634-638 (2001).

7. Tepass, U. Crumbs, a component of the apical membrane, is required for zonula adherens formation in primary epithelia of Drosophila. Dev.Biol. 177, 217-225 (1996).

8. Klebes, A. \& Knust, E. A conserved motif in Crumbs is required for E-cadherin localisation and zonula adherens formation in Drosophila. Curr.Biol. 10, 76-85 (2000).

9. Hong, Y., Ackerman, L., Jan, L. Y., \& Jan, Y. N. Distinct roles of Bazooka and Stardust in the specification of Drosophila photoreceptor membrane architecture. Proc.Natl.Acad.Sci.U.S.A 100, 12712-12717 (2003).
10. Izaddoost, S., Nam, S. C., Bhat, M. A., Bellen, H. J., \& Choi, K. W. Drosophila Crumbs is a positional cue in photoreceptor adherens junctions and rhabdomeres. Nature 416, 178-183 (2002).

11. Pellikka, M. et al. Crumbs, the Drosophila homologue of human CRB1/RP12, is essential for photoreceptor morphogenesis. Nature 416, 143-149 (2002).

12. Wodarz, A., Hinz, U., Engelbert, M., \& Knust, E. Expression of crumbs confers apical character on plasma membrane domains of ectodermal epithelia of Drosophila. Cell 82, 67-76 (1995).

13. Mehalow, A. K. et al. CRB1 is essential for external limiting membrane integrity and photoreceptor morphogenesis in the mammalian retina. Hum.Mol. Genet. 12, 2179-2189 (2003).

14. Van de Pavert, S. A. et al. Crumbs homologue 1 is required for maintenance of photoreceptor cell polarization and adhesion during light exposure. J.Cell Sci. 117, 4169-4177 (2004).

15. Jacobson, S. G. et al. Crumbs homolog 1 (CRB1) mutations result in a thick human retina with abnormal lamination. Hum.Mol.Genet. 12, 1073 1078 (2003).

16. Jensen, A. M., Walker, C., \& Westerfield, M. mosaic eyes: a zebrafish gene required in pigmented epithelium for apical localization of retinal cell division and lamination. Development 128, 95-105 (2001).

17. Wei, X., Zou, J., Takechi, M., Kawamura, S., \& Li, L. Nok plays an essential role in maintaining the integrity of the outer nuclear layer in the zebrafish retina. Exp.Eye Res. 83, 31-44 (2006).

18. Wei, X. \& Malicki, J. nagie oko, encoding a MAGUK-family protein, is essential for cellular patterning of the 
retina. Nat.Genet. 150-157 (2002).

19. Jensen, A. M. \& Westerfield, M. Zebrafish mosaic eyes is a novel FERM protein required for retinal lamination and retinal pigmented epithelial tight junction formation. Curr.Biol. 14, 711-717 (2004).

20. Parra, M. et al. Differential domain evolution and complex RNA processing in a family of paralogous EPB41 (protein 4.1) genes facilitate expression of diverse tissue-specific isoforms. Genomics 84, 637-646 (2004).

21. Reid, M. E., Takakuwa, Y., Conboy, J., Tchernia, G., \& Mohandas, N. Glycophorin $C$ content of human erythrocyte membrane is regulated by protein 4.1. Blood 75, 2229-2234 (1990).

22. Takakuwa, Y., Tchernia, G., Rossi, M., Benabadji, M., \& Mohandas, N. Restoration of normal membrane stability to unstable protein 4.1-deficient erythrocyte membranes by incorporation of purified protein 4.1. J.Clin.Invest 78, 80-85 (1986).

23. Kamberov, E. et al. Molecular cloning and characterization of Pals, proteins associated with mLin-7. J.Biol.Chem. 275, 11425-11431 (2000).

24. Hoover, K. B. \& Bryant, P. J. Drosophila Yurt is a new protein-4.1-like protein required for epithelial morphogenesis. Dev. Genes Evol. 212, 230-238 (2002).

25. Laprise, P. et al. The FERM Protein Yurt Is a Negative Regulatory Component of the Crumbs Complex that Controls Epithelial Polarity and Apical Membrane Size. Dev.Cell 11, 363-374 (2006).

26. Hsu, Y. C., Willoughby, J. J., Christensen, A. K., \& Jensen, A. M. Mosaic Eyes is a novel component of the Crumbs complex and negatively regulates photoreceptor apical size. Development 133, 4849-4859 (2006).
27. Kantardzhieva, A. et al. MPP5 recruits MPP4 to the CRB1 complex in photoreceptors. Invest Ophthalmol.Vis.Sci. 46, 2192-2201 (2005).

28. Frangioni, J. V. \& Neel, B. G. Solubilization and purification of enzymatically active glutathione S-transferase (pGEX) fusion proteins. Anal.Biochem. 210, 179187 (1993).

29. Takeuchi, K., Kawashima, A., Nagafuchi, A., \& Tsukita, S. Structural diversity of band 4.1 superfamily members. J.Cell Sci. 107 ( Pt 7), 1921-1928 (1994).

30. Mburu, P. et al. Whirlin complexes with p55 at the stereocilia tip during hair cell development. Proc.Natl.Acad.Sci. U.S.A 103, 10973-10978 (2006).

31. Lemmers, C. et al. CRB3 binds directly to Par6 and regulates the morphogenesis of the tight junctions in mammalian epithelial cells. Mol.Biol.Cell 15, 1324-1333 (2004).

32. Le Bivic, A. et al. An internal deletion in the cytoplasmic tail reverses the apical localization of human NGF receptor in transfected MDCK cells. J.Cell Biol. 115, 607-618 (1991).

33. van Wijk, E. et al. The DFNB31 gene product whirlin connects to the Usher protein network in the cochlea and retina by direct association with USH2A and VLGR1. Hum.Mol.Genet. 15, 751-765 (2006).

34. Roh, M. H. et al. The Maguk protein, Pals1, functions as an adapter, linking mammalian homologues of Crumbs and Discs Lost. J.Cell Biol. 157, 161-172 (2002).

35. Grimm, C. et al. Isolation and embryonic expression of the novel mouse gene Hic1, the homologue of HIC1, a candidate gene for the Miller-Dieker syndrome. Hum.Mol.Genet. 8, 697-710 (1999). 
36. den Hollander, A. I. et al. Isolation of Crb1, a mouse homologue of Drosophila crumbs, and analysis of its expression pattern in eye and brain. Mech.Dev. 110, 203-207 (2002).

37. Pearson, M. A., Reczek, D., Bretscher, A., \& Karplus, P. A. Structure of the ERM protein moesin reveals the FERM domain fold masked by an extended actin binding tail domain. Cell 101, 259-270 (2000).

38. Parra, M. et al. Cloning and characterization of 4.1G (EPB41L2), a new member of the skeletal protein 4.1 (EPB41) gene family. Genomics 49, 298306 (1998).

39. Turunen, O., Wahlstrom, T., \& Vaheri, A. Ezrin has a COOH-terminal actin-binding site that is conserved in the ezrin protein family. J.Cell Biol. 126, 1445-1453 (1994).

40. Garcia-Garcia, M. J. et al. Analysis of mouse embryonic patterning and morphogenesis by forward genetics. Proc.Natl.Acad.Sci.U.S.A 102, 5913-5919 (2005).

41. Lee, J. D., Silva-Gagliardi, N. F., Tepass, U., McGlade, C. J., \& Anderson, $\mathrm{K}$. V. The FERM protein Epb4.115 is required for organization of the neural plate and for the epithelial-mesenchymal transition at the primitive streak of the mouse embryo. Development 134, 20072016 (2007).

42. van Rossum, A. G. et al. Pals1/Mpp5 is required for correct localization of $\mathrm{Crb1}$ at the subapical region in polarized Muller glia cells. Hum.Mol.Genet. 15, 2659-2672 (2006).

43. Hoover, K. B. \& Bryant, P. J. The genetics of the protein 4.1 family: organizers of the membrane and cytoskeleton. Curr.Opin.Cell Biol. 12, 229-234 (2000).

44. Marfatia, S. M., Leu, R. A., Branton, D., \& Chishti, A. H. Identification of the protein 4.1 binding interface on glyco- phorin $\mathrm{C}$ and $\mathrm{p} 55$, a homologue of the Drosophila discs-large tumor suppressor protein. J.Biol.Chem. 270, 715-719 (1995).

45. Biederer, T. \& Sudhof, T. C. CASK and protein 4.1 support F-actin nucleation on neurexins. J.Biol.Chem. 276, 47869-47876 (2001).

46. Cohen, A. R. et al. Human CASK/ LIN-2 binds syndecan-2 and protein 4.1 and localizes to the basolateral membrane of epithelial cells. J.Cell Biol. 142, 129-138 (1998).

47. Roh, M. H., Liu, C. J., Laurinec, S., \& Margolis, B. The carboxyl terminus of zona occludens-3 binds and recruits a mammalian homologue of discs lost to tight junctions. J.Biol.Chem. 277, 2750127509 (2002).

48. Hurd, T. W., Gao, L., Roh, M. H., Macara, I. G., \& Margolis, B. Direct interaction of two polarity complexes implicated in epithelial tight junction assembly. Nat.Cell Biol. 5, 137-142 (2003).

49. Straight, S. W. et al. Loss of PALS1 expression leads to tight junction and polarity defects. Mol.Biol.Cell15, 19811990 (2004). 



\section{CHAPTER 6}

\section{Composition and function of the Crumbs protein complex in the mammalian retina.}

Ilse Gosens ${ }^{1}$, Anneke I. den Hollander ${ }^{1}$, Frans P. M. Cremers ${ }^{1}$, Ronald Roepman ${ }^{1}$

${ }^{1}$ Department of Human Genetics, Nijmegen Centre for Molecular Life Sciences, Radboud University Nijmegen Medical Centre, Geert Grooteplein Zuid 10, P.O. Box 9101, 6500 HB, Nijmegen, The Netherlands.

Provisionally accepted for publication in Experimental Eye Research 



\section{ABSTRACT}

The transmembrane protein Crumbs is essential in defining the apical membrane domain in embryonic epithelia as well as in photoreceptors. Crumbs proteins are conserved between species and their intracellular domains are involved in organizing a macromolecular protein scaffold. In the mammalian retina, CRB1 is involved in maintenance of cell-to-cell adhesion. Recently, new binding partners of the Crumbs protein complex have been identified, including members of the membrane palmitoylated protein family (MPP). MPP proteins consist of several protein-protein interaction domains that are used to assemble macromolecular complexes, in part by homo- and heterodimerization. Identification of novel components of the Crumbs complex will lead to a more profound understanding of its function in different cellular processes in health and disease. This review summarizes the current view on the composition and function of the Crumbs protein complex in the mammalian retina. The core Crumbs complex localizes apical to the outer limiting membrane, where photoreceptors and Müller glia contact each other. Correct functioning of Crumbs ensures adhesion between these cells by an unknown mechanism. Some components of the complex are found to exert a function in the photoreceptor synapses and/or at the region of the connecting cilium. Moreover, studies using polarized cell cultures or model organisms, like Drosophila and zebrafish, allow us to hypothesize on yet concealed links to several biological processes in the mammalian eye involving Crumbs, including retinal patterning, ciliogenesis and vesicular transport. 


\section{INTRODUCTION}

The Crumbs gene was initially identified in Drosophila and encodes a large transmembrane protein that defines the apical membrane of embryonic epithelial cells ${ }^{1}$. Besides a function in maintenance of apico-basal cell polarity and adherens junctions in embryonic epithelia, Crumbs has a similar function in adult fly retina ${ }^{2}$. For epithelial cells and neurons like photoreceptors, separation of their apical and basal compartments is critical for correct functioning in cell-to-cell adhesion, intercellular signaling, directional transport of (secreted) molecules and correct tissue formation. Crumbs molecules are found in many species, ranging from invertebrates to mammals ${ }^{3}$. The intracellular domain is highly conserved and is involved in organizing a macromolecular protein scaffold at the interior of the different cell types to exert its function. In this review we describe the components of the intracellular Crumbs protein complex and we focuss on their (putative) links to different cellular processes in the mammalian retina.

\subsection{Crumbs and retinal degeneration}

Abnormal Crumbs function has been associated with different types of retinal degeneration in humans ${ }^{4}$. Mutations in Crumbs homologue 1 (CRB1) cause autosomal recessive Leber congenital amaurosis (LCA), several subtypes of autosomal recessive retinitis pigmentosa (RP) and autosomal dominant pigmented paravenous chorioretinal atrophy ${ }^{5-8}$. LCA is the most severe retinal dystrophy, characterized by blindness or severe visual impairment at birth, while RP is considered milder with a later age of onset. No genotype-phenotype correlation could be established for CRB1 mutations, since nonsense mutations on both alleles have been identified in LCA and RP patients ${ }^{9}$. This suggests that other genes might influence the severity of the disease.

CRB1-associated LCA is suggested to be caused by a developmental defect in the retina, since LCA patients with mutations in CRB1 have a thickened retina and lack the distinct layering of a fully developed adult retina, resembling a more immature normal retina ${ }^{10}$. Retinal patterning defects have also been observed in the zebrafish retina associated with mutations in different members of the Crumbs complex, e.g. nok ${ }^{11}$, ome ${ }^{12,13}$, has $^{12}$, glo ${ }^{12,14}$ and moe ${ }^{15}$ mutants, encoding MPP5 ${ }^{11,16}, \mathrm{Crb} 2 \mathrm{a}{ }^{17}$, aPKC ${ }^{18}$, N-cadherin ${ }^{19}$ and EPB41L5/YMO1 ${ }^{20,21}$ proteins , respectively.

In RP, rod photoreceptors are first affected leading to night blindness, followed by progressive death of cone photoreceptors, restricting 
the visual field initially in the periphery and in later stages can lead to complete loss of vision. Progressive photoreceptor cell death could be due to loss of adhesion between Müller glia cells and photoreceptors ${ }^{22}$. Interestingly, a targeted knock-out mouse model that completely lacks Crumbs has a relatively mild defect. The retinas are initially normal, but at 3-9 months the Crb1 knockout retinas develop localized lesions due to the loss of interaction between photoreceptors and Müller glia cells. The $\mathrm{CRB} / \mathrm{Crb}$ complex localizes to the outer limiting membrane (OLM) at the subapical region (SAR), a region apical to the adherens junctions. Adherens junctions at the OLM form the adhesion site where photoreceptors connect to each other and to Müller glia cells. Total lack of Crumbs in these mice leads to neuronal cell death in the inner and outer nuclear layers of the retina after loss of OLM integrity in localized lesions. Upon moderate exposure to light, the number of severe focal retinal lesions significantly increases in the $\mathrm{Crb1}^{-/}$retina. A targeted knock-in mouse with a RP12 missense mutation (Cys249Trp) in the extracellular sixth calcium-binding epidermal growth factor domain of Crb1) showed loss of photoreceptors in the retina relatively late compared with mice lacking Crb1 ${ }^{23}$. In addition, this missense mutation was associated with a downregulation of mitotic checkpoint protein Pttg1.

In a naturally occurring mouse mutant ( $r d 8$ mouse), a single base deletion in $C r b 1$ causes a frameshift resulting in a premature stop codon at amino acid residue 1207 . The resulting protein, if produced, lacks the transmembrane and cytoplasmic regions and is predicted to be secreted 24. The observed effects include a zonula adherens (ZA) that is discontinuous and fragmented, as well as shortened photoreceptor inner and outer segments as early as 2 weeks after birth. This suggests a developmental defect in these structures rather than a degenerative process and resembles the findings in Drosopbila, where lack of Crumbs leads to shortened rhabdomere and stalk membranes, the functional equivalents of vertebrate outer and inner segments, respectively ${ }^{25}$. Interestingly, phenotypic variability of the Crb1 mutation was observed in different genetic backgrounds, confirming that other genes/factors can influence the phenotype.

\subsection{Conservation of Crumbs}

In humans and mice, three Crumbs orthologues are detected: CRB1/ crb1, CRB2/crb2 and CRB3/crb3. Crb1 is only expressed in the brain and retina ${ }^{26}$, whereas CRB2/Crb2 is also expressed in the kidney, RPE/cho- 
roid and at low levels in heart, lung and placenta ${ }^{27}$. Crb3 is ubiquitously expressed in different (epithelial derived) tissues, including the retina ${ }^{28}$. In zebrafish, there are five Crumbs isoforms due to whole genome duplication: crb1, crb2a/ome, crb2b, crb3a and crb3b ${ }^{17}$. CRB1/crb1 and $\mathrm{CRB} 2 / \mathrm{crb} 2$ comprise of a large extracellular part with several epidermal growth factor (EGF)-like repeats (some are predicted to bind calcium) and laminin A globular domain (G)-like repeats, which are also identified in Drosophila Crumbs (Fig. 1). Differential splicing of CRB1/crb1 and $\mathrm{CRB} 2 / \mathrm{crb} 2$ lead to truncated forms that lack the transmembrane and intracellular part, and are hypothesized to be secreted, e.g. CRB1b (Fig. 1) ${ }^{27}$. In mice, an additional short isoform, Crb1s, is detected ${ }^{29}$. CRB3/crb3 proteins lack the large extracellular region, but do contain the transmembrane and intracellular part. The intracellular part contains two protein binding motifs, the FERM binding and PDZ binding motif (or PBM), that are highly conserved in all orthologues and across all species (Fig. 1) ${ }^{3}$. In addition, a phosphorylation motif is present in the intracellular domain of Drosophila Crumbs ${ }^{30}$, of which some residues are conserved in human CRBs. Recently, an alternative splice form of CRB3 is described that has a different 23-amino acid sequence at its $\mathrm{C}$-terminus ending in the sequence CLPI (CRB3-CLPI, fig. 1) ${ }^{31}$. This splice form is found in human, cow, mouse, rat and dog, but not in Drosophila and zebrafish.

Almost all mutations in human CRB1 are identified in the extracellular part, and encompass frameshifts, nonsense and missense mutations. Most of the frameshift and nonsense mutations are predicted to lead to nonsense-mediated decay or to truncate the protein such that the transmembrane and intracellular part are lacking. Missense mutations affecting cysteine residues in the laminin AG-like domains or EGF-like repeats are predicted to affect the secondary structure of the protein by disrupting disulfide bridges ${ }^{9}$. Laminin AG-like or ALPS (agrin, laminin, perlecan and slit) domains have been identified in several proteins and could be involved in protein-protein interactions ${ }^{32}$, as could EGF-like domains ${ }^{33}$. Some of the EGF-like domains in CRB1 are predicted to bind calcium. In fibrillin-1, a protein that contains calcium binding EGF-like domains, calcium is predicted to stiffen the protein into a rod-like structure and thereby promoting protein-protein interaction or protecting against proteolytic cleavage ${ }^{34-36}$. Several CRB1 missense mutations localize to the EGF-like domains and the majority involves highly conserved residues that could affect protein folding. Indeed, NMR studies on recombinant wild-type and $\mathrm{N} 1317 \mathrm{H}$ (missense mutation found in LCA patients) CRB1 fragments structure demonstrated that $\mathrm{Ca}^{2+}$ binding is altered, leading to 
disruption of interactions between adjacent EGF domains ${ }^{37}$. One missense variation is described in the intracellular domain, but it is unclear whether it is associated with retinal disease ?

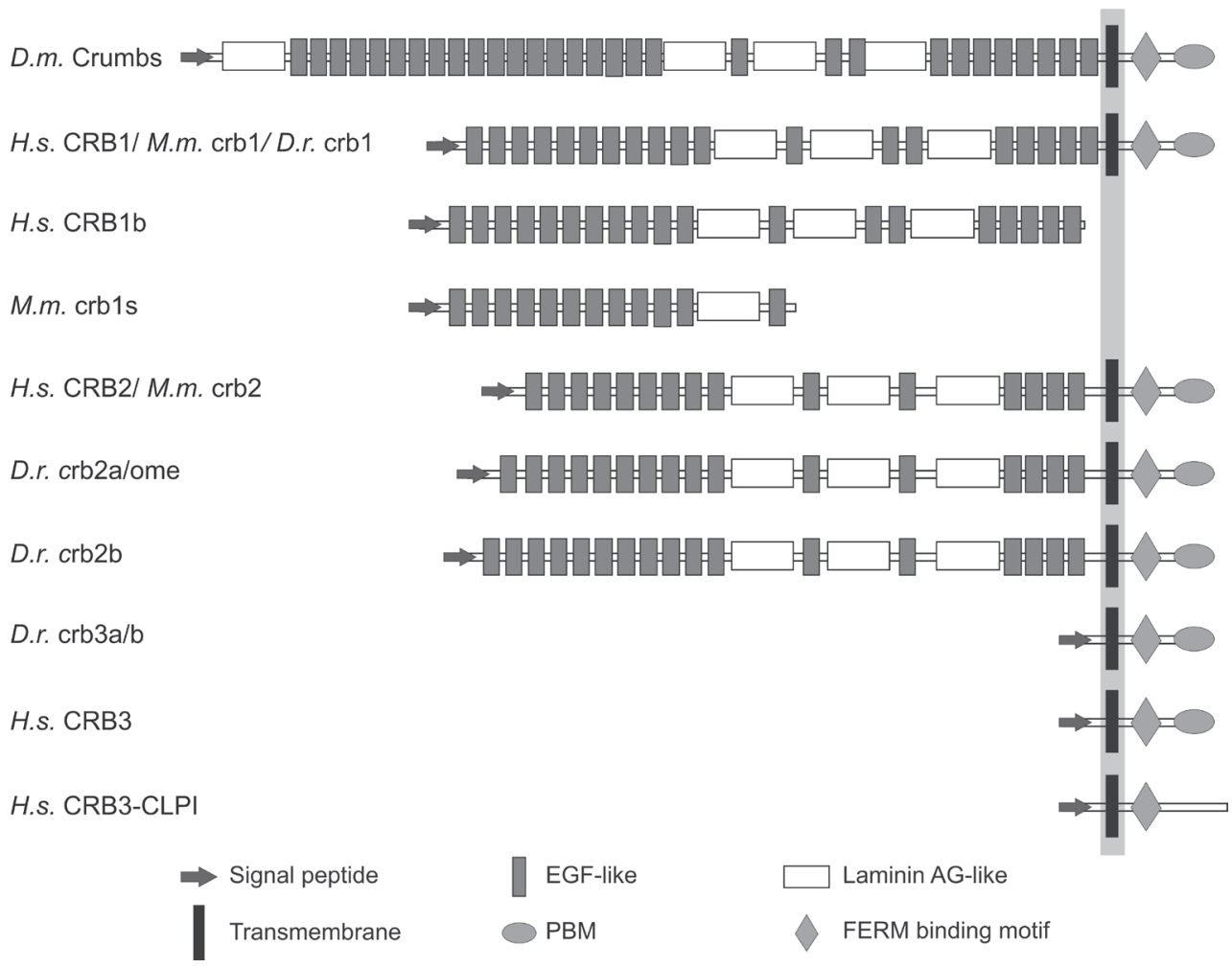

Fig. 1 Crumbs orthologues and paralogues in several species.

Drosophila (D.m.) Crumbs contains a signal peptide, 30 EGF-like domains, four laminin AG-like repeats, a transmembrane (TM) domain, and an intracellular part that contains the conserved FERM binding and PDZ binding motif (PBM). Human (bomo sapiens, H.s.), mouse (mus musculus, M.m.) and zebrafish (danio rerio, D.r.) CRB1/crb1 have an identical domain composition of 19 EGFlike repeats, three laminin AG-like repeats, a TM domain and the conserved intracellular motifs. A splice form that lacks the TM and intracellular domain is detected in human (H.s. CRB1b) and is predicted to be secreted. In mice, an additional small isoform is detected (crb1s) in skin. Human, mouse and zebrafish CRB2/crb2 all contain a transmembrane and intracellular conserved binding motif, but differ slightly in their extracellular domain composition in comparison to each other and to CRB1. CRB3/crb3 lacks the extracellular EGF-like and laminin AG-like domains, but the intracellular binding motifs are retained. An alternative CRB3 splice form is detected in humans and other mammals including rodents with a divergent $\mathrm{C}$-terminal sequence ending in the amino acids CLPI (CRB3-CLPI). 
On the protein level, CRB proteins migrate at a larger molecular weight than predicted due to post-translational modifications. The extracellular domain contains conserved N-linked glycosylation motifs, likely to be responsible for the $\mathrm{N}$-glycosylation of CRB1 and CRB3 ${ }^{28}$ (Chapter 2). An actual function for this decoration with carbohydrates is unknown, although transmembrane proteins generally do not protrude uncovered from the exterior of a eukaryotic cell. Whether or not carbohydrate binding proteins such as lectins have affinity for Crumbs is not known, since no binding partners for the extracellular domain have been identified so far.

\section{The intracellular Crumbs protein complex}

The 37-amino acid intracellular domain of Crumbs is critical for its function in organizing a protein scaffold. The terminal four amino acids, the ERLI motif or PDZ binding motif (PBM) mediates binding to MPP5 (membrane palmitoylated protein 5). CRB1 and MPP5 form a complex in the mammalian retina as determined by immunoprecipitation from mouse retinal lysates ${ }^{22}$ and by a GST-pull down assay from bovine retinal lysates (Fig. 2). The ERLI motif is indispensable for interaction, since a GST-fusion of the intracellular domain of CRB1 that lacks the ERLI motif abolishes the interaction with MPP5 (Fig. 2). MPP5 homologues in Drosophila (Stardust) and zebrafish (nok) are able to interact with the PBM of Crumbs in the same manner ${ }^{21,38,39}$.

Recently, an interacting protein for the second conserved motif, the FERM binding motif (GTY sequence), was identified in several species. In Drosophila, FERM domain protein Yurt was found to bind to Crumbs, and zebrafish homologue YMO1 interacts directly with Crumbs and Nok ${ }^{21,40}$. Binding of the mammalian homologue EPB41L5 to all CRBs in the mammalian retina involves the intracellular GTY motif, as removal of this motif leads to loss of interaction (Fig. 2). Parallel to the interactions identified in zebrafish, EPB41L5 is able to bind to the HOOK domain of MPP5, indicating a conserved importance of the CrumbsMPP5-EPB41L5 protein complex in different species. Co-expression and co-localisation analysis revealed not only an important function of this complex in the retina, but also in other epithelial derived tissues, such as the kidney (Chapter 5).

A GST-pull down experiment from bovine retinas using the intracellular domain of CRB1 added two multi-PDZ proteins, PATJ and MUPP1 to the complex, albeit indirectly via the PDZ domain of MPP5. 
Removal of the ERLI binding motif in CRB1 disrupted these interactions, whereas removal of the FERM motif did not (Fig. 2).

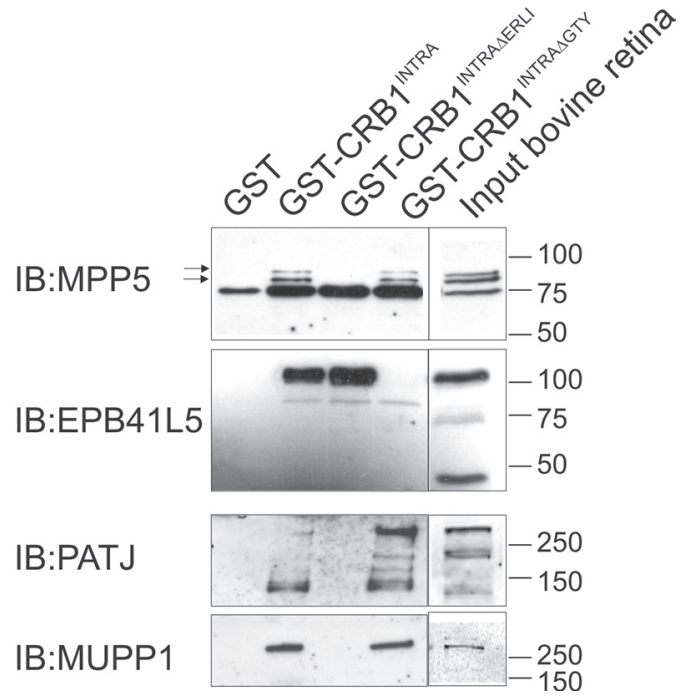

Fig. 2 The Crumbs protein scaffold in the retina identified by GST-pull down analysis.

The intracellular domain of CRB1 fused to GST was able to pull down MPP5 (detected as a doublet around $78 \mathrm{kD}$, indicated with arrows), EPB41L5, PATJ and MUPP1 from bovine retinal lysates. Removal of the ERLI motif abolished the binding of MPP5, PATJ and MUPP1, whereas lack of the FERM binding motif (GTY sequence) had no effect. FERM domain EPB41L5 binds to the GTY sequence, since removal leads to loss of interaction. Removing the ERLI motif had no effect on binding. GST was used as a negative control. IB, immunoblot staining with antibodies against proteins as indicated.

Besides PDZ domains (8 or 10 in PATJ, depending on the isoform, and

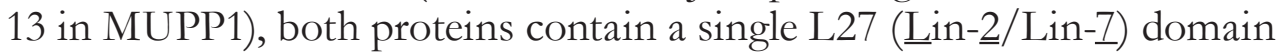
that binds to the N-terminal L27 domain of MPP5 via L27 domain heterodimerization ${ }^{41,42}$. Studies using polarized epithelial cells demonstrated that PATJ is involved in targeting the Crumbs-MPP5 complex to the apical membrane at tight junctions ${ }^{41}$. PATJ and MUPP1 are known to bind to tight junction proteins. For PATJ this includes binding to ZO-3 and claudin ${ }^{43}$, and MUPP1 associates with ZO-1 and ZO-2 via binding of claudin and JAM-A (junctional adhesion molecule) (Fig. 3) ${ }^{44}$. ZO-1 binds directly to F-actin ${ }^{45-47}$ and to ZO-2 ${ }^{48,49}$, and both proteins associate with actin binding protein EPB $41{ }^{50}$, connecting this part of the Crumbs complex to the cortical cytoskeleton in epithelial cells. In cells that lack 
well developed tight junctions, ZO-1 was found in adherens junctions as well ${ }^{51}$.

Whether PATJ and MUPP1 are involved in targeting the CRBMPP5 complex at the SAR, or that their binding partners provide a connection to the actin cytoskeleton in the retina remains to be determined. MUPP1 and PATJ are detected at the SAR, together with MPP5 and CRBs, although MUPP1 was previously identified in an immunoprecipitate from mouse retinal lysates, whereas PATJ was not ${ }^{22}$. In Drosophila, DPATJ is necessary for the proper positioning of adherens junctions in developing photoreceptors and prevents late-onset (light-induced) photoreceptor degeneration ${ }^{52,53}$.

\subsection{MPP family of proteins}

In the retina, the Crumbs protein complex is expanded through additional connections with scaffold protein MPP5 (Fig. 3). MPP5, also known as PALS1, belongs to the membrane palmitoylated protein (MPP) family that consists of seven members. The MPP family of proteins is widely represented in the Crumbs complex. MPP5 binds directly to family members MPP1, MPP3 and MPP4 in the retina ${ }^{54}$ (and chapter 2 and 3), and recently MPP5 was found together with MPP7 in a tight junctional complex in epithelial cells ${ }^{55}$. MPP proteins are members of the larger membrane-associated guanylate kinase (MAGUK) protein family and share a basic domain structure (Fig. 4). The so-called MAGUK module

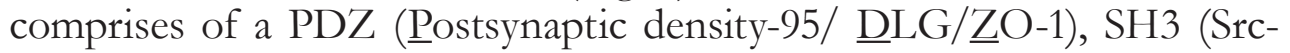
homology-3) and GUK (guanylate kinase) domain. Some members have a lysine rich sequence motif, also known as a HOOK domain, between the SH3 and the catalytically inactive GUK domain. Furthermore, the family prototype MPP1 (or p55) lacks the L27 domains that are present in the remaining MPPs.

MPP proteins can assemble in an intra- or intermolecular fashion via a SH3-GUK domain swapping mechanism ${ }^{56}$ (and chapter 2 and 3). The SH3 domain of MPP5 binds to its own GUK domain and to that of MPP1 and MPP4. Similarly, the SH3-GUK domains of MPP1 and MPP4 can fold intramolecularly. However, the SH3 domains of MPP1/ MPP4 do not interact with the GUK domain of MPP5, showing that the domain swapping mechanism is directional and does not allow all possible binding combinations. Deletion construct analysis suggests that the GUK domain of MPP3 can bind to MPP5 using the same binding mechanism as MPP1 and MPP4 ${ }^{54}$. By means of the SH3-GUK interaction between MPPs, oligomerization into larger complexes that could in- 


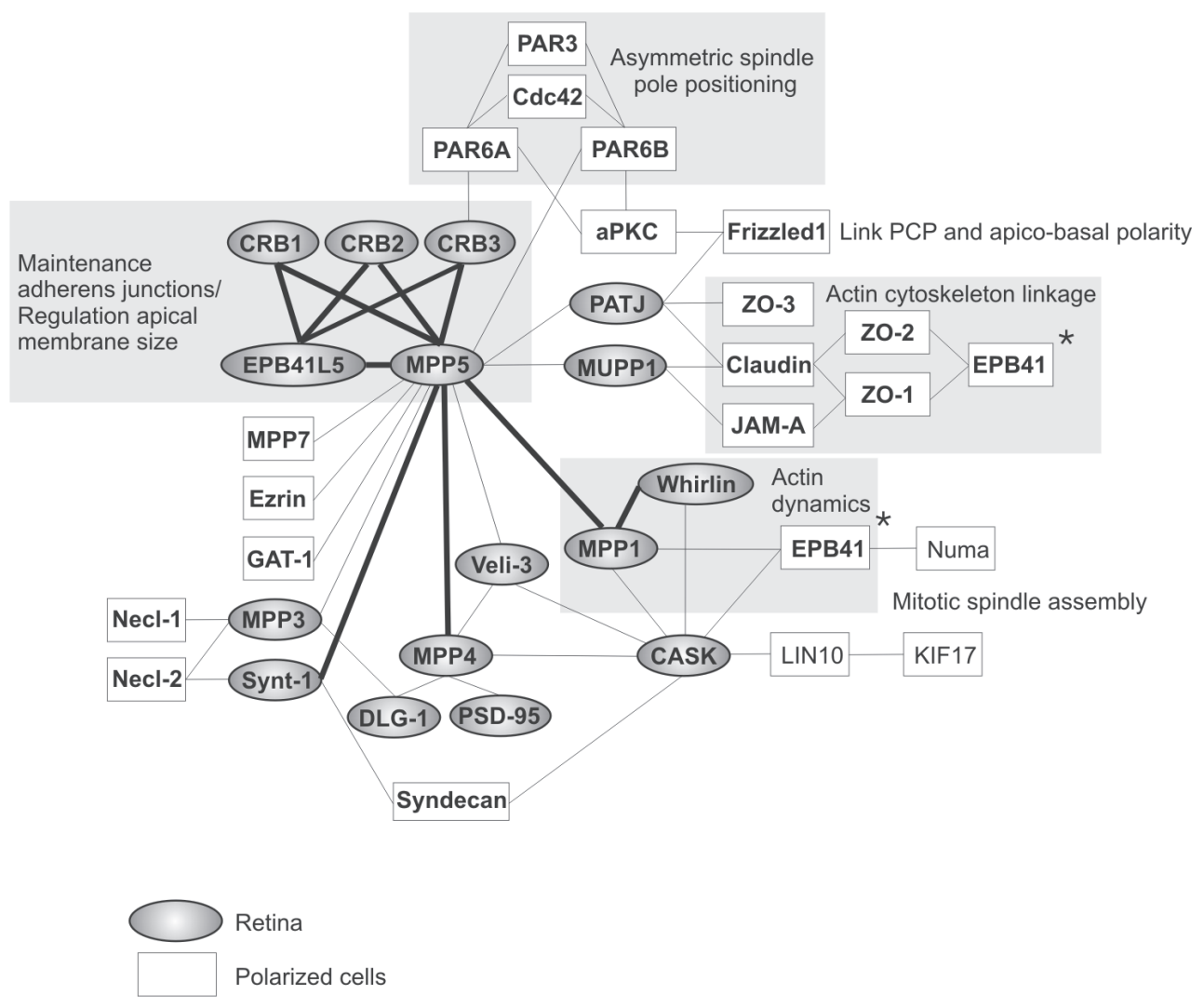

Fig. 3 Schematic illustration of the composition of the Crumbs protein complex.

Proteins that are present in retinal complexes are indicated by gray ellipses. White squares represent proteins that bind to Crumbs protein complex members, but the connections are identified in polarized cells other than retinal cells. Note that the complete downstream complexes are not depicted to avoid complication. The gray boxes indicate the general cellular processes in which parts of the protein complex have been implicated. Synt-1 is syntenin-1, Necl-1 or -2 is nectin-like 1 or $2 .{ }^{*}$ EPB41 is implicated in actin cytoskeletal linkage and actin dynamics which is seperated here for easy visual representation.

Bold lines connect proteins of which their interaction is described by work presented in this thesis.

tegrate cell signals is feasible, as has been described for other MAGUKs such as PSD-95, CASK and DLG1 ${ }^{56-58}$. This specific binding mechanism of MPP proteins provide a means for regulating the assembly of protein connections at the region of the OLM or at the synapses and could help to orchestrate intracellular signaling complexes, although the exact nature of the signals involved is still unclear. 


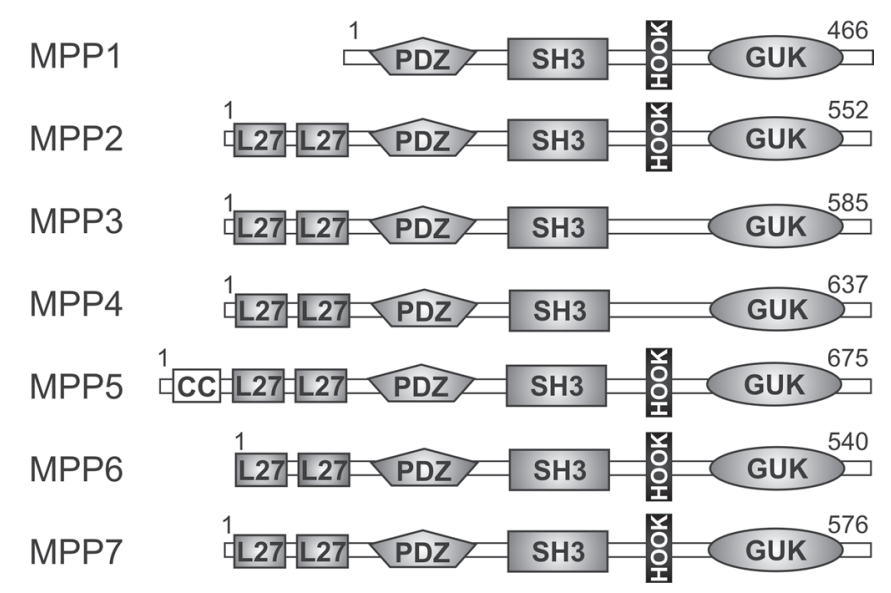

Fig. 4 Schematic domain composition of MPP proteins.

MPP proteins consist of two conserved L27 domains (except for MPP1), a PDZ, SH3 and GUK domain. Some have an additional HOOK domain and MPP5 has an additional coiled-coiled (CC) region.

Besides the SH3 and GUK domain, additional motifs of MPP5 are involved in protein-protein interactions, allowing the build-up of an even more complex protein scaffold. The C-terminal L27 domain binds to LIN-7 ${ }^{59}$, promoting protein stability in polarized epithelial cells ${ }^{60}$. The $\mathrm{N}$-terminal region containing the coiled-coil domain and part of the first L27 binds to PAR6 in epithelial cells ${ }^{61}$. PAR6 is not detected at the SAR in the mouse retina ${ }^{22}$, although it plays an important role in the morphogenesis of photoreceptors in Drosophila ${ }^{62}$. Ezrin, an FERM domain containing actin binding protein of the membrane-cytoskeleton linker family, binds to an unidentified domain in the C-terminal part of MPP5 in gastric parietal cells ${ }^{63}$, while the PDZ domain of MPP5 binds to GABA transporter GAT-1, stabilizing GAT-1 levels in GABAergic neurons of the CNS ${ }^{64}$. Whether ezrin and GAT-1 bind similarly to MPP5 in the retina remains to be investigated. MPP7 was recently found to be associated with MPP5 in a complex, although the interaction might not be direct ${ }^{55}$. The mRNA transcript is expressed in the retina, but the localization of this protein remains elusive (Chapter 3).

\section{The function of the Crumbs complex at the SAR in the retina}

The core of the Crumbs protein complex, consisting of CRB1, CRB2, CRB3 and its direct interactors EPB41L5 and MPP5, as well as its associated MPP protein scaffold are found at the subapical region (SAR), 
a region just apical to the adherens junctions at the outer limiting membrane (OLM) 22,54 (and chapter 2 and 3). Adherens junctions are the sites where the cytoplasmic face of the plasma membrane is attached to actin filaments. They are found in photoreceptors and Müller cells, as well as in epithelial cells, where they separate the apical and basolateral membrane. Mammalian epithelial cells develop a tight junction (TJ), a specialized plasma membrane microdomain apical to the AJ, forming a seal between adjacent cells preventing passage of molecules from one side of the epithelial sheet to another. Although Drosophila epithelial and mammalian photoreceptors likewise do not form tight junctions, their SAR harbors protein complexes that appear also at tight junctions in mammalian epithelial cells (Fig. 5). In addition, Drosophila epithelial cells have septate junctions (SJ) that are located basal to the ZA and form a region of close membrane contacts that extends over large parts of the lateral plasma membrane domain ${ }^{22,65,66}$.

Immuno-electron microscopy in murine retinas revealed that Crb1 immunoreactivity is mainly present at the SAR in Müller glia cells, whereas CRB2, CRB3, PATJ, MPP5 and MUPP1 are present both in photoreceptors and Müller glia cells ${ }^{67}$. MPP5 is involved in targeting CRB1 to the correct site of action. When MPP5 is downregulated by siRNA in Müller glia, CRB1 as well as CRB2, CRB3, MUPP1 and Veli3 mislocalize at the OLM. Whether MPP5 downregulation also affects localization of MPP1, MPP3 and MPP4 at the OLM remains to be determined.

Conflicting findings were reported for the subcellular localization of MPP4, the only MPP protein that is synthesized specifically in the retina. The localization depended on the antibodies used, the applied method for antibody epitope retrieval as well as the species that was studied. In human, bovine and porcine retinas, MPP4 localizes to the region of the OLM at the SAR in addition to the synapses of photoreceptors ${ }^{68}$ (and chapter 2). In bovine and porcine, MPP4 is present at the region of the connecting cilium in photoreceptors and in the outer plexiform layer (OPL), inner plexiform layer (IPL) and optic fiber layer ${ }^{68}$, while in mice, MPP4 was detected at the OLM in small amounts in some studies ${ }^{69}$ (and chapter $\mathrm{x}$ ), and only at the OPL in others ${ }^{70,71}$. Loss of Mpp4 function in $\mathrm{MPP}^{-/}$mice only sporadically causes photoreceptor displacement, without changing the Crumbs protein complex at the OLM, adherens junctions or synapse structure. This suggests that MPP4 does not have an essential docking/scaffolding function at these locations, or that its absence can be easily compensated by other MPP proteins ${ }^{69,72}$. In addition, scanning laser ophthalmology revealed no retinal degeneration ${ }^{69}$. 


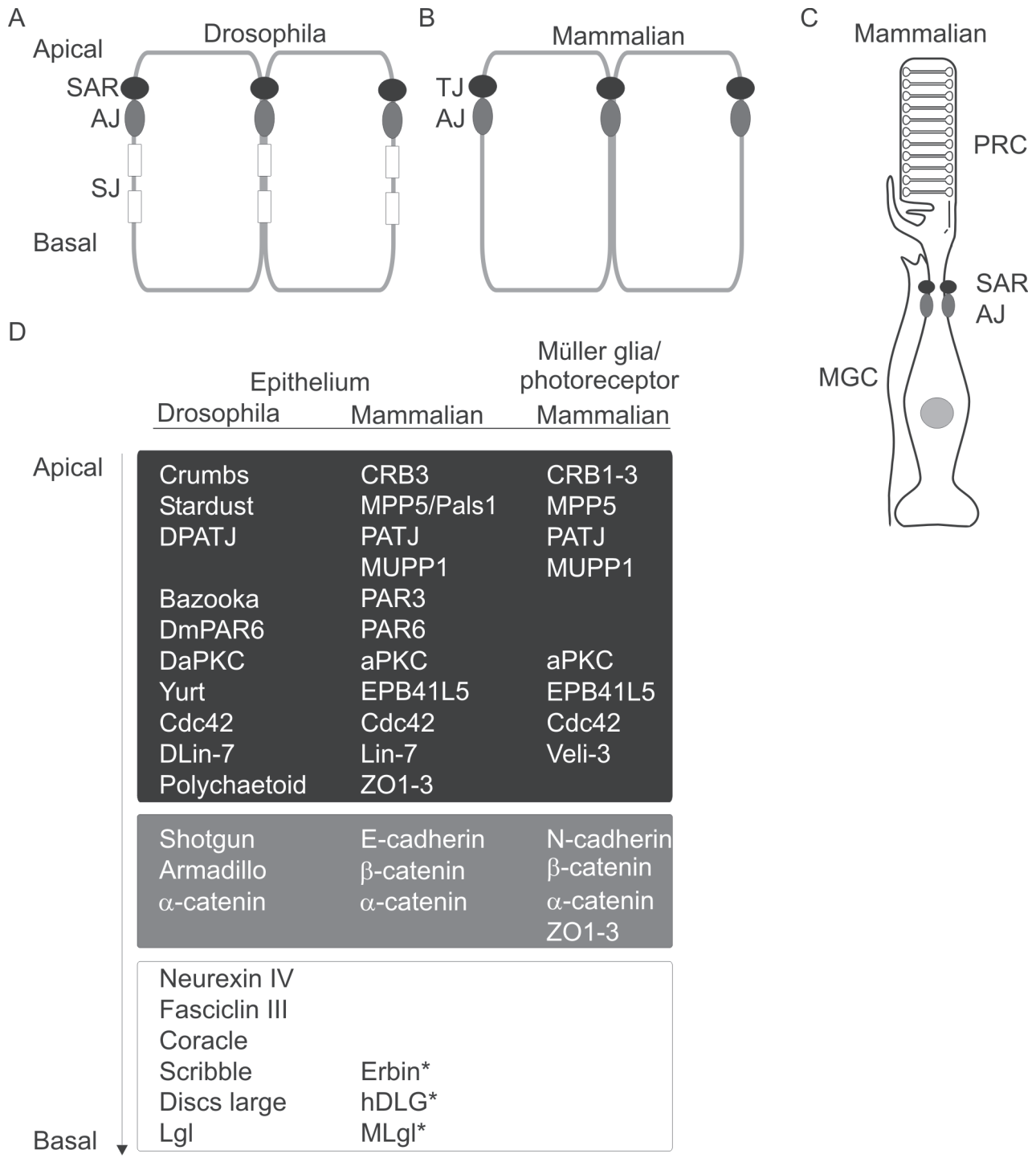

Fig. 5 Comparison of junctional organization in the Drosophila epithelium, mammalian epithelium and mammalian photoreceptor and/or Müller cell.

A: Drosophila epithelial cells have a subapical region (SAR) apical to adherens junctions (AJ) as well as septate junctions (SJ). B: Mammalian epithelial cells have tight junctions (TJ) apical to the AJ. C: Rod mammalian photoreceptor cells lack TJ, but have a SAR apical to adherens junctions. D: Overview of the molecules that are found at the SAR/TJ (black box), AJ (gray box) and SJ (white box). * Mammalian epithelial cells do not have septate junctions, but these Drosophila orthologues are found at the basal membrane. 
Besides a role of Crumbs in maintenance of cell-cell adhesion in the differentiated adult retina, studies using zebrafish as a model have revealed retinal patterning defects during development associated with mutations in Crumbs complex members. Zebrafish nok ${ }^{11}$, ome ${ }^{12,13}$, has ${ }^{12}, g{ }^{12,14}$ and moe ${ }^{15}$ mutants indicate that the associated proteins, MPP5 ${ }^{11,16}, \mathrm{Crb} 2 \mathrm{a}{ }^{17}$, $\mathrm{aPKC}^{18}, \mathrm{~N}$-cadherin ${ }^{73}$ and EPB41L5/YMO1 respectively ${ }^{20,40}$, are crucial for correct retinal integrity. Remarkably, in these mutants all neuronal cell types have developed from the neuroepithelium. During development, retinal cell types migrate away from the apical surface of the neuroepithelium to form layers of specific cell classes. The apical junctions of the neuroepithelium continue to exist in the adult retina as the OLM, forming a layer of cell adhesive contacts between photoreceptors and Müller glia cells. During neurogenesis, loss of epithelial polarity, already in the neuroepithelium, could result in an incorrect separation or growth of the apical and basolateral domains, thus causing improper distribution of cues and affecting the position of postmitotic cells ${ }^{74}$.

In a conditional knockout mouse model for aPKC, proper retinal lamination failed to arise in post-mitotic differentiating neurons ${ }^{75}$. The progenitor cells did not generate proper adhesion with photoreceptors and photoreceptor differentiation occurred at different locations in the retina. The photoreceptors showed morphological defects and failed to form ribbon synapses. APKC is a member of the apical PAR3/PAR6/ aPKC protein complex ${ }^{76}$, that functions in establishing cell polarity in epithelial cells and links to the Crumbs complex via CRB3-PAR6A and MPP5-PAR6B protein interactions ${ }^{61,77,78}$.

In zebrafish, localization of Crumbs apical to the OLM is linked with the formation of photoreceptor apical membrane. Knockdown of zebrafish Crb2b leads to a dramatic reduction in inner segment size ${ }^{21}$. This function of Crumbs in apical membrane size determination is controlled by FERM protein YMO1 or EPB41L5 ${ }^{21}$. A similar effect is seen in the Drosophila Yurt mutant, where lack of EPB41L5 leads to an expansion of photoreceptor apical membrane in a Crumbs-dependent manner ${ }^{40}$. Overexpression of Drosophila Crumbs produces an expansion of photoreceptor apical membrane as well ${ }^{25}$, while a lack of Crumbs reduces the rhabdomere and stalk membrane length ${ }^{2}$. In mammals, Crumbs function in membrane size regulation remains tentative. In $r d 8$ mice that lack partial Crb1 function, a reduction in inner segment length is seen, while in total absence of $\mathrm{Crb} 1$ in the $\mathrm{Crb1}^{-/}$knockout mice this is not observed. 


\subsection{The Crumbs protein complex in synapses}

The Crumbs complex also seems to play an important role in the synaptic layer of the photoreceptors. Although CRB1 and MPP5 were not detected in the photoreceptor synapses (Chapter 2), a pan-CRB antibody does place CRB2 and/or CRB3 at this location (Fig. 6). In addition, MPP1, MPP3 and MPP4 were detected at this subcellular site ${ }^{22,54}$ MPP4 affects the turnover rate of scaffold protein PSD-95, while LIN-7 or Veli-3, binding partner of MPP4 and MPP5 in the mammalian retina ${ }^{70}$, is also affected by the absence of MPP4 in the synapse. At the OLM, Veli-3 is not affected, probably because MPP5 is still present. MPP4 functions as a presynaptic complex organizer in photoreceptors that includes stabilization of PSD-95 and proper localization of plasma membrane calcium ATPases (PMCAs), thereby regulating calcium homeostasis and synaptic transmission in rods ${ }^{69,72}$. For MPP1 and MPP3, no transmembrane protein or calcium channel binding has been identified in the retina, although MPPs that belong to the MAGUK family of proteins generally are implicated in organizing scaffold to integrate signaling pathway components ${ }^{79}$.
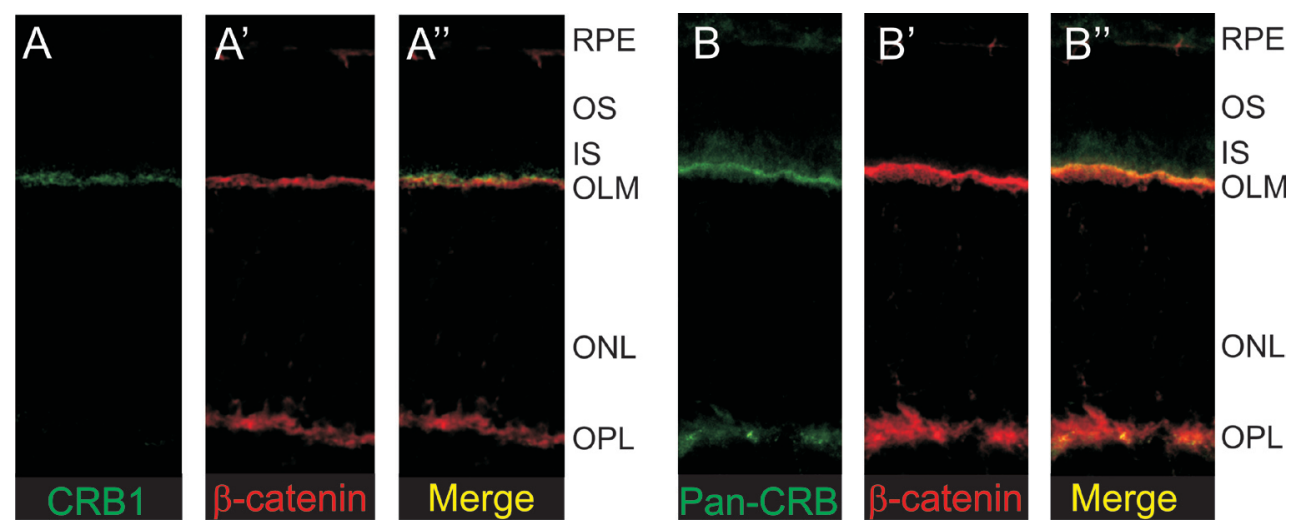

Fig. 6 Immunohistochemistry of CRB proteins in the retina.

A: CRB1 localizes to the OLM and is not found in the synapses. B: An antibody that recognizes all three CRB orthologues (pan-CRB) stains the OLM and the OPL. Immunohistochemistry was performed as described in addendum 2 .

The SAR and the synapses of photoreceptors thus seem to contain an overlapping content of Crumbs complex molecules. Although in the mammalian retina no tight junctions are formed, similar proteins can be found in TJ of mammalian epithelial cells and the SAR of the mammalian photoreceptor (Fig. 5). It is interesting to note that tight junctions share functional and structural features with synapses. Both structures 
are linked to the cadherin/nectin based adhesion system in the adherens junctions ${ }^{80-83}$, are intimately linked to the actin cytoskeleton ${ }^{84,85}$ and contain satellite Golgi systems for local protein synthesis ${ }^{86-88}$. Both present MAGUK proteins at their protein scaffolds ${ }^{89,90}$, and multi PDZ-domain protein MUPP1 is even found at tight junctions, the SAR as well as glutaminergic terminals ${ }^{22,44,91}$. Recently, a proteomic and bio-informatics approach to study the protein content of epithelial tight junctions revealed a cluster of synaptic proteins ${ }^{86}$. This implicates that some components of the Crumbs complex that are found at the SAR and at synapses may play a role in similar cellular processes that involve organization of large protein scaffolds to regulate cell-cell communication and adhesion between Müller glia and photoreceptors as well as between neurons, most likely between the postsynaptic and the presynaptic membrane.

\subsection{The Crumbs protein complex in cilia and basal bodies}

Besides displacement of cell junctions in the neuroepithelium, Crb2b, aPKC, N-cadherin and MPP5 zebrafish mutants display mislocalized centrosomes ${ }^{11,13,14,18}$. The centrosome has classically been implicated as microtubule organization centre (MTOC) that regulates cell division in meiotic and mitotic cells. During mitosis, organization of the spindle pole orientation determines the outcome of cell division. If the mitotic spindle pole is aligned parallel to the apical surface, both cells inherit a portion of the apical membrane (planar cell division), while a mitotic spindle pole axis perpendicular to the surface of the retinal neuroepithelium, results in the inheritance of functional components in one daughter cell and the other one migrates away to differentiate (asymmetric cell division) ${ }^{92,93}$. The centrosome and the basal body, a structure highly related to centrioles, the components of a centrosome, as well as a cilium are recognized as interlinked both structurally through a network of microtubules and functionally by similar protein content with overlapping functions. Furthermore, exit of the cell cycle is accompanied by ciliary assembly (reviewed in reference 94). The neuroepithelium has a single cilium, with $\mathrm{Crb}$ staining at the base where basal bodies are located. In zebrafish, crb2b and crb3a morphants have shorter and displaced cilia. Crb2b is necessary for elongation and motility of polynephric cilia, while crb3a functions in regulating the length of the auditory hair cell kinocilium ${ }^{17}$. Whether CRBs are found at the basal body and/or connecting cilium in mammalian photoreceptors, a structure that is formed between the inner and outer segments and functions in exchange of molecules 
between the two compartments, remains to be determined. However, CRB3 has recently been reported to localize to cilia in the kidney, and loss of CRB3 blocks cilia formation in cultured epithelial kidney cells ${ }^{95}$. Recently, an additional CRB3 splice form with a alternative $\mathrm{C}$-terminus that lacks the ERLI motif is described in human and rodents ${ }^{31}$. This form localises to the cilia membrane and a membrane compartment at the mitotic spindle poles and is suggested to play a role in ciliogenesis and cell division. Based on the fact that CRB1 is mainly expressed in Müller glia cells ${ }^{67}$, and above presented findings, mammalian CRB2 and CRB3 are the most likely candidates to be found in the photoreceptor basal body and/or connecting cilium.

In addition, Crumbs complex member MPP1 is found in the kinocilium and basal bodies of hair cells ${ }^{96}$ and connecting cilium and basal bodies in the retina, where it co-localizes with PDZ scaffold molecule whirlin (Chapter 3). The involvement of whirlin in actin filament polymerization ${ }^{97,98}$, and binding of MPP1 to FERM domain protein EPB41 involved in anchoring molecules to the actin cytoskeleton ${ }^{96,99}$, elicit a role for both proteins in actin organization and dynamics. Actin filaments are required for proper positioning of ciliary basal bodies ${ }^{100,101}$ and both molecules may be indispensable in this process, besides generation or maintenance of cell polarity through a link with MPP5 (Chapter 3).

The Crumbs associated PAR6/PAR3/aPKC complex localizes to cilia and regulates ciliogenesis in mammalian epithelial cells ${ }^{95}$. PAR6, in general, plays a role in the organization of the microtubule cytoskeleton and the localization of the MTOC during asymmetric cell division or migration ${ }^{102}$. Mitotic spindle orientation during retinal progenitor cell divisions requires aPKC function in vertebrates ${ }^{18}$.

PATJ was recently associated with a function in reorientation of MTOC and affects the direction of migration in epithelial cells ${ }^{103}$. More interestingly, DPATJ binds to Frizzled 1, recruits aPKC and influences planar cell polarity in the Drosophila eye ${ }^{104}$.

During development, CRBs, MPP5 and EPB41L5 are expressed in the ventricular layer of the mouse neural tube in proliferative cells and not in postmitotic neural compartments suggesting a putative role in cell division and differentiation (Chapter 5). In zebrafish mutants, loss of adhesion and mislocalized centrosomes in the neuroepithelium precede neurogenesis. Patterning defects seem to arise without affecting differentiation in retinal cell types. In mammals it is not known whether defects in the neuroepithelium could give rise to retinal degeneration, although 
in LCA patients patterning defects have been observed ${ }^{10}$. In it interesting to note that in Drosophila polarized epithelia, Crumbs-mediated adherens junction adhesion is coupled to the outcome of cell division. Adherens junctions are found to inhibit asymmetric cell division in the epithelium by planar cell polarity cues. RNA inference to inhibit Crumbs function led to misoriented spindle poles and loss of epithelial integrity. Overexpression of the intracellular domain of Crumbs is sufficient to disrupt the formation of adherens junctions and to provoke a switch from symmetric to asymmetric cell division ${ }^{105}$. Whether a similar mechanism is at the base of mammalian neuroepithelium cell divisions or could explain patterning defects in the vertebrate retina, is not known.

\subsection{The Crumbs complex and vesicular transport}

The Crumbs protein complex has been described to play a role in regulation of cell polarity in the retina, controlling aspects ranging from maintenance of cell-cell adhesion to apical membrane size determination. These functions are exerted apically to the OLM, but depending on the Crumbs orthologue and specific binding partners, additional functions of the complex at photoreceptor synapses and cilia structures are detected. Although participation of the Crumbs protein complex is observed in several processes at different locations, it is noteworthy to mention that a common denominator in the regulation of these processes supports a role for the Crumbs complex in vesicle transport. Several studies synthesize data showing the importance of Crumbs in endocytotic vesicle transport.

Recently, multiple signaling pathways that control cell polarity are found to be regulated by endocytosis of transmembrane proteins. Blocking the intracellular pathway that normally leads to degradation of the endocytosed transmembrane receptors in the lysosome ultimately leads to loss of cell polarity ${ }^{106}$. Crumbs is presumably delivered by vesicles to the apical surface of epithelial cells, where it specifies the apical domain. Its expression is regulated by Rab5-dependent endocytosis, since a defect in Rab5 function leads to abnormal apical expansion in Drosophila epithelial cells ${ }^{107}$.

Furthermore, fast disassembly of apical junctions that is seen under normal physiological conditions and in pathologic events, cannot solely be explained by down regulation of the expression of proteins. The junction-remodeling event can take place in 1 hour, while the half-life of junctional proteins can be up to ten times as long. Endocytosis of junc- 
tional protein complexes serve as an additional more rapid mechanism by which remodeling is controlled ${ }^{108}$. The Rich/Amot complex, important in maintenance of tight junction in polarized epithelial cells, is found in a dynamic complex of interactions with MPP5, PATJ and PAR3 proteins that is regulated by the small GTPase Cdc42. This complex might regulate the uptake of polarity proteins at the tight junctions, since selective internalization of MPP5 and PAR3 is seen upon Amot overexpression and might provide a new sorting mechanism that decides whether tight junction transmembrane proteins are recycled back to the plasma membrane or are sent elsewhere such as lysosomes or the trans-Golgi network 55,109. PATJ was found to regulate specifically CRB3 distribution in intracellular compartments such as the early endosomes upon downregulation in polarized epithelial cells ${ }^{110}$.

Could the Crumbs protein complex play a role in similar endocytotic vesicular transport processes in the retina? Although in the retina, no tight junctions are found, the similarity in protein components that build tight junctions and regulated its maintenance that are found at the SAR is striking. Furthermore, at synapses intensive vesicular transport is needed to ensure proper signal transmission and several members of the Crumbs complex are detected there.

In addition, tandem PDZ domain protein syntenin-1 was found to interact with MPP5 in the mammalian retina (Chapter 4). Syntenin-1 might form a molecular link between the adherens junction complex and Crumbs complex at the apical site. In addition, syntenin-1 was found to co-localize with Arf6 and Rab11 and is known to regulate surface availability of transmembrane receptors ${ }^{111}$.

\section{Animal models for Crumbs and Crumbs complex members}

Several animal models have been studied and have given insight into the pathology of Crumbs defects (table 1). It appears that CRBs can substitute each other in at least some developmental or cellular processes and thus share functional characteristics. Remarkably, in zebrafish mutants as well as in LCA patients retinal lamination defects have been observed. Drosophila mutants and the naturally occurring Crb mouse model both present developmental defects in photoreceptor apical membrane morphogenesis, which was also observed for the Crb2b zebrafish morphants. However, there are also discrepancies between species in the severity of the phenotypes, the time of onset and the tissues that are affected (table 
1). Differences in phenotypic features observed in mice and human have been observed before, for example in a model for Usher syndrome: shaker mice with mutations in MyosinVIIa only present hearing loss, while humans suffer from deafness and blindness (Usher syndrome type B) ${ }^{112,113}$. On the other hand, environmental factors or genetic modifiers could influence the differential outcome of a disease. In $r d 8$ mice, the severity of the phenotype depends on the genetic background ${ }^{24}$.

Table 1. Overview of animal models for Crumbs and its complex members

\begin{tabular}{|c|c|c|c|}
\hline Organism & Model $^{\text {ref }}$ & Mutation/ defect & Phenotype \\
\hline Fruitfly & 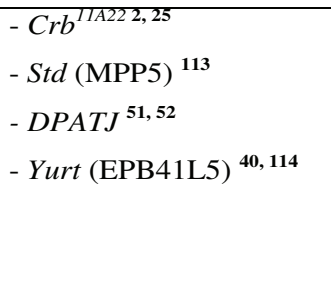 & $\begin{array}{l}\text { - Null, eye clones } \\
\text { - Null, eye clones } \\
\text { - Null, eye clones } \\
\text { - Null }\end{array}$ & $\begin{array}{l}\text { - Fragmented ZA, reduced } \\
\text { rhabdomere length, reduced stalk } \\
\text { membrane length. } \\
\text { - Defects in epithelial morphogenesis, } \\
\text { expansion of photoreceptor apical } \\
\text { domain }\end{array}$ \\
\hline Zebrafish & $\begin{array}{l}\text { - Crb2b morphant }^{17} \\
\text { - Crb3a morphant }{ }^{17} \\
\text { - Ome (Crb2a) }{ }^{\mathbf{1 3}, 17} \\
\text { - Nok (MPP5) }{ }^{\mathbf{1 1 , 1 6}} \\
\text { - Moe }(\mathrm{EPB} 41 \mathrm{~L} 5)^{\mathbf{1 5 , 2 0 , 2 1}}\end{array}$ & $\begin{array}{l}\text { - Morpholino } \\
\text { - Morpholino } \\
\text { - Loss of function } \\
\text { - Loss of function } \\
\text { - Loss of function }\end{array}$ & $\begin{array}{l}\text { - Reduced inner segments/ cilia length } \\
\text { - Reduced kinocilium length } \\
\text { - Retinal lamination defects } \\
\text { - Retinal lamination defects } \\
\text { - Retinal lamination defects }\end{array}$ \\
\hline Mouse & 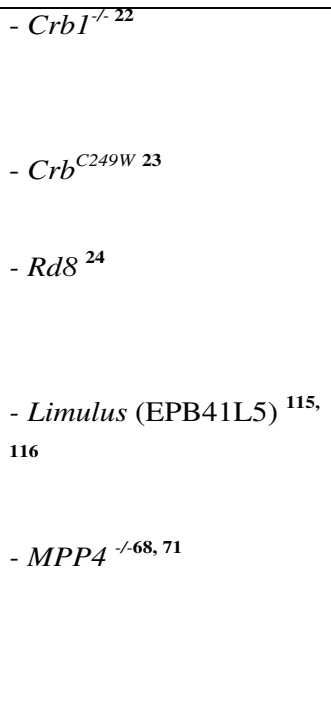 & $\begin{array}{l}\text { - Knock-out, no } \\
\text { functional Crb1 } \\
\text { - Knock-in, RP } \\
\text { mutation } \\
\text { - Naturally } \\
\text { occurring, lacks } \\
\text { TM and intra } \\
\text { - Knock-out, null } \\
\text { - Knock-out }\end{array}$ & $\begin{array}{l}\text { - Lesions with fragmented ZA, (half)- } \\
\text { rosettes, neuronal cell death in INL } \\
\text { and ONL after 3-9 months. } \\
\text { - Late onset loss of photoreceptors. } \\
\text { - Fragmented ZA, shortened inner and } \\
\text { outer segments, retinal folds and } \\
\text { pseudorosettes } 2 \text { weeks after birth. } \\
\text { - Morphogenetic defects of the } \\
\text { mesoderm, endoderm and neural } \\
\text { plate. } \\
\text {-Destabilization presynaptic protein } \\
\text { complex, disturbed calcium } \\
\text { homeostasis and synaptic } \\
\text { transmission. No retinal degeneration. }\end{array}$ \\
\hline
\end{tabular}


It is tempting to speculate that defects in members of the Crumbs protein complex may be involved in the pathogenesis. More detailed analysis of Crumbs orthologues or even isoforms as well as the composition of the protein complex during development will lead to a more in-depth insight into Crumbs function in the retina and will shed more light on the mechanism that leads to retinal degeneration in humans.

\section{CONCLUSION}

The Crumbs protein complex is implicated in mechanisms that control cell polarity. Evidently, Crumbs is indispensable for adhesion and adherens junction maintenance at the OLM region of the mammalian retina. Its intracellular protein scaffold plays a role in the organization of membrane domains by defining the apical compartment. The presence of MPP proteins in the Crumbs complex suggests the build-up of a macromolecular complex that could couple this transmembrane protein to downstream intracellular effectors and increase the efficiency and specificity in signal transduction. Although signaling pathways have not yet been described in the retina, a conserved phosphorylation site in the intracellular domain of Crumbs and a link to PCP signaling is noted.

Some Crumbs orthologues as well as interactors of Crumbs have been implicated in basal body and cilia related processes. Although it is too early to say that mammalian Crumbs proteins influence asymmetric cell division and/or migration, associations with these important processes have been described. Finally, involvement of components of the Crumbs complex in vesicular transport and regulation of cell polarity by influencing endocytosis is a recent finding and will evidently lead to more research in this direction. 


\section{REFERENCES}

1. Tepass, U., Theres, C., \& Knust, E. crumbs encodes an EGF-like protein expressed on apical membranes of Drosophila epithelial cells and required for organization of epithelia. Cell 61, 787-799 (1990).

2. Izaddoost, S., Nam, S. C., Bhat, M. A., Bellen, H. J., \& Choi, K. W. Drosophila Crumbs is a positional cue in photoreceptor adherens junctions and rhabdomeres. Nature 416, 178-183 (2002).

3. Richard, M. et al. Towards understanding CRUMBS function in retinal dystrophies. Hum.Mol.Genet. 15 Spec No 2, R235-R243 (2006).

4. den Hollander, A. I. et al. Mutations in a human homologue of Drosophila crumbs cause retinitis pigmentosa (RP12). Nat.Genet. 23, 217-221 (1999).

5. den Hollander, A. I. et al. Leber congenital amaurosis and retinitis pigmentosa with Coats-like exudative vasculopathy are associated with mutations in the crumbs homologue 1 (CRB1) gene. Am.J.Hum.Genet. 69, 198-203 (2001).

6. Lotery, A. J. et al. Mutations in the CRB1 gene cause Leber congenital amaurosis. Arch.Ophthalmol. 119, 415-420 (2001).

\section{Lotery, A. J. et al. CRB1 mutations} may result in retinitis pigmentosa without para-arteriolar RPE preservation. Ophthalmic Genet. 22, 163-169 (2001).

8. McKay, G. J., Clarke, S., Davis, J. A., Simpson, D. A., \& Silvestri, G. Pigmented paravenous chorioretinal atrophy is associated with a mutation within the crumbs homolog 1 (CRB1) gene. Invest Ophthalmol.Vis.Sci. 46, 322-328 (2005).

9. den Hollander, A. I. et al. CRB1 mutation spectrum in inherited retinal dystrophies. Hum.Mutat. 24, 355-369 (2004).

10. Jacobson, S. G. et al. Crumbs ho- molog 1 (CRB1) mutations result in a thick human retina with abnormal lamination. Hum.Mol.Genet. 12, 1073 1078 (2003).

11. Wei, X. \& Malicki, J. nagie oko, encoding a MAGUK-family protein, is essential for cellular patterning of the retina. Nat.Genet. 150-157 (2002).

12. Malicki, J. et al. Mutations affecting development of the zebrafish retina. Development 123, 263-273 (1996).

13. Malicki, J. \& Driever, W. oko meduzy mutations affect neuronal patterning in the zebrafish retina and reveal cell-cell interactions of the retinal neuroepithelial sheet. Development 126, 1235-1246 (1999).

14. Pujic, Z. \& Malicki, J. Mutation of the zebrafish glass onion locus causes early cell-nonautonomous loss of neuroepithelial integrity followed by severe neuronal patterning defects in the retina. Dev.Biol. 234, 454-469 (2001).

15. Jensen, A. M., Walker, C., \& Westerfield, M. mosaic eyes: a zebrafish gene required in pigmented epithelium for apical localization of retinal cell division and lamination. Development 128, 95-105 (2001).

16. Wei, X., Zou, J., Takechi, M., Kawamura, S., \& Li, L. Nok plays an essential role in maintaining the integrity of the outer nuclear layer in the zebrafish retina. Exp.Eye Res. 83, 31-44 (2006).

17. Omori, Y. \& Malicki, J. oko meduzy and Related crumbs genes are determinants of apical cell features in the vertebrate embryo. Curr.Biol. 16, 945-957 (2006).

18. Horne-Badovinac, S. et al. Positional cloning of heart and soul reveals multiple roles for PKC lambda in zebrafish organogenesis. Curr.Biol. 11, 1492-1502 (2001). 
19. Malicki, J., Jo, H., \& Pujic, Z. Zebrafish $\mathrm{N}$-cadherin, encoded by the glass onion locus, plays an essential role in retinal patterning. Dev.Biol. 259, 95-108 (2003).

20. Jensen, A. M. \& Westerfield, M. Zebrafish mosaic eyes is a novel FERM protein required for retinal lamination and retinal pigmented epithelial tight junction formation. Curr.Biol. 14, 711-717 (2004).

21. Hsu, Y. C., Willoughby, J. J., Christensen, A. K., \& Jensen, A. M. Mosaic Eyes is a novel component of the Crumbs complex and negatively regulates photoreceptor apical size. Development 133, 4849-4859 (2006).

22. van de Pavert, S. A. et al. Crumbs homologue 1 is required for maintenance of photoreceptor cell polarization and adhesion during light exposure. J.of Cell Sci. 117, 4169-4177 (2004).

23. van de Pavert, S. A. et al. A single amino acid substitution (Cys249Trp) in Crb1 causes retinal degeneration and deregulates expression of pituitary tumor transforming gene Pttg1. J.Neurosci. 27, 564-573 (2007).

24. Mehalow, A. K. et al. CRB1 is essential for external limiting membrane integrity and photoreceptor morphogenesis in the mammalian retina. Hum.Mol. Genet. 12, 2179-2189 (2003).

25. Pellikka, M. et al. Crumbs, the Drosophila homologue of human CRB1/RP12, is essential for photoreceptor morphogenesis. Nature 416, 143-149 (2002).

26. den Hollander, A. I. et al. Isolation of Crb1, a mouse homologue of Drosophila crumbs, and analysis of its expression pattern in eye and brain. Mech.Dev. 110, 203-207 (2002).

27. van den Hurk, J. A. J. M. et al. Characterization of the Crumbs homolog 2 (CRB2) gene and analysis of its role in retinitis pigmentosa and Leber congenital amaurosis. Mol.Vis. 11, 263-273 (2005).

28. Makarova, O., Roh, M. H., Liu, C. J., Laurinec, S., \& Margolis, B. Mammalian Crumbs 3 is a small transmembrane protein linked to protein associated with Lin-7 (Pals1). Gene 302, 21-29 (2003).

29. Watanabe, T., Miyatani, S., Katoh, I., Kobayashi, S., \& Ikawa, Y. Expression of a novel secretory form (Crb1s) of mouse Crumbs homologue Crb1 in skin development. Biochem.Biophys.Res.Commun. 313, 263-270 (2004).

30. Sotillos, S., az-Meco, M. T., Caminero, E., Moscat, J., \& Campuzano, S. DaPKC-dependent phosphorylation of Crumbs is required for epithelial cell polarity in Drosophila. J.Cell Biol. 166, 549-557 (2004).

31. Fan, S. et al. A novel Crumbs3 isoform regulates cell division and ciliogenesis via importin $\{$ beta\} interactions. J.Cell Biol. (2007).

32. Timpl, R. et al. Structure and function of laminin LG modules. Matrix Biol. 19, 309-317 (2000).

33. Selander-Sunnerhagen, M. et al. How an epidermal growth factor (EGF)-like domain binds calcium. High resolution NMR structure of the calcium form of the NH2-terminal EGF-like domain in coagulation factor X. J.Biol.Chem. 267, 19642-19649 (1992).

34. Downing, A. K. et al. Solution structure of a pair of calcium-binding epidermal growth factor-like domains: implications for the Marfan syndrome and other genetic disorders. Cell 85, 597605 (1996).

35. Smallridge, R. S. et al. Solution structure and dynamics of a calcium binding epidermal growth factor-like domain pair from the neonatal region of human fibrillin-1. J.Biol.Chem. 278, 12199-12206 (2003). 
36. Reinhardt, D. P., Ono, R. N., \& Sakai, L. Y. Calcium stabilizes fibrillin-1 against proteolytic degradation. J.Biol. Chem. 272, 1231-1236 (1997).

37. Davis, J. A., Handford, P. A., \& Redfield, C. The N1317H substitution associated with Leber congenital amaurosis results in impaired interdomain packing in human CRB1 EGF domains. J.Biol.Chem. (2007).

38. Hong, Y., Stronach, B., Perrimon, N., Jan, L. Y., \& Jan, Y. N. Drosophila Stardust interacts with Crumbs to control polarity of epithelia but not neuroblasts. Nature 414, 634-638 (2001).

39. Bachmann, A., Schneider, M., Theilenberg, E., Grawe, F., \& Knust, E. Drosophila Stardust is a partner of Crumbs in the control of epithelial cell polarity. Nature 414, 638-643 (2001).

40. Laprise, P. et al. The FERM Protein Yurt Is a Negative Regulatory Component of the Crumbs Complex that Controls Epithelial Polarity and Apical Membrane Size. Dev.Cell 11, 363-374 (2006).

41. Roh, M. H. et al. The Maguk protein, Pals1, functions as an adapter, linking mammalian homologues of Crumbs and Discs Lost. J.Cell Biol. 157, 161-172 (2002).

42. Lemmers, C. et al. hINADl/PATJ, a homolog of discs lost, interacts with crumbs and localizes to tight junctions in human epithelial cells. J.Biol.Chem. 277, 25408-25415 (2002).

43. Roh, M. H., Liu, C. J., Laurinec, S., \& Margolis, B. The carboxyl terminus of zona occludens- 3 binds and recruits a mammalian homologue of discs lost to tight junctions. J.Biol.Chem. 277, 2750127509 (2002).

44. Hamazaki, Y., Itoh, M., Sasaki, H., Furuse, M., \& Tsukita, S. Multi-PDZ domain protein 1 (MUPP1) is concentrated at tight junctions through its possible interaction with claudin-1 and junctional adhesion molecule. J.Biol.Chem. 277, 455461 (2002).

45. Fanning, A. S., Jameson, B. J., Jesaitis, L. A., \& Anderson, J. M. The tight junction protein ZO-1 establishes a link between the transmembrane protein occludin and the actin cytoskeleton. J.Biol. Chem. 273, 29745-29753 (1998).

46. Fanning, A. S., Ma, T. Y., \& Anderson, J. M. Isolation and functional characterization of the actin binding region in the tight junction protein $\mathrm{ZO}$ 1. FASEB J. 16, 1835-1837 (2002).

47. Itoh, M., Nagafuchi, A., Moroi, S., \& Tsukita, S. Involvement of ZO-1 in cadherin-based cell adhesion through its direct binding to alpha catenin and actin filaments. J.Cell Biol. 138, 181-192 (1997).

48. Itoh, M. et al. Direct binding of three tight junction-associated MAGUKs, ZO-1, ZO-2, and ZO-3, with the $\mathrm{COOH}$ termini of claudins. J.Cell Biol. 147, 1351-1363 (1999).

49. Fanning, A. S., Jameson, B. J., Jesaitis, L. A., \& Anderson, J. M. The tight junction protein ZO-1 establishes a link between the transmembrane protein occludin and the actin cytoskeleton. J.Biol. Chem. 273, 29745-29753 (1998).

50. Mattagajasingh, S. N., Huang, S. C., Hartenstein, J. S., \& Benz, E. J., Jr. Characterization of the interaction between protein 4.1R and ZO-2. A possible link between the tight junction and the actin cytoskeleton. J.Biol.Chem. 275, 3057330585 (2000).

51. Itoh, M. et al. The $220-\mathrm{kD}$ protein colocalizing with cadherins in non-epithelial cells is identical to ZO-1, a tight junction-associated protein in epithelial cells: cDNA cloning and immunoelectron microscopy. J.Cell Biol. 121, 491-502 (1993).

52. Richard, M., Grawe, F., \& Knust, E. DPATJ plays a role in retinal morpho- 
genesis and protects against light-dependent degeneration of photoreceptor cells in the Drosophila eye. Dev.Dyn. 235, 895-907 (2006).

53. Nam, S. C. \& Choi, K. W. Domainspecific early and late function of Dpatj in Drosophila photoreceptor cells. Dev. Dyn. 235, 1501-1507 (2006).

54. Kantardzhieva, A., Alexeeva, S., Versteeg, I., \& Wijnholds, J. MPP3 is recruited to the MPP5 protein scaffold at the retinal outer limiting membrane. FEBS J. 273, 1152-1165 (2006).

55. Wells, C. D. et al. A Rich1/Amot complex regulates the Cdc42 GTPase and apical-polarity proteins in epithelial cells. Cell 125, 535-548 (2006).

56. Nix, S. L., Chishti, A. H., Anderson, J. M., \& Walther, Z. hCASK and hDlg associate in epithelia, and their src homology 3 and guanylate kinase domains participate in both intramolecular and intermolecular interactions. J.Biol.Chem. 275, 41192-41200 (2000).

57. McGee, A. W. et al. Structure of the SH3-guanylate kinase module from PSD-95 suggests a mechanism for regulated assembly of MAGUK scaffolding proteins. Mol.Cell 8, 1291-1301 (2001).

58. McGee, A. W. \& Bredt, D. S. Identification of an intramolecular interaction between the $\mathrm{SH} 3$ and guanylate kinase domains of PSD-95. J.Biol.Chem. 274, 17431-17436 (1999).

59. Kamberov, E. et al. Molecular cloning and characterization of Pals, proteins associated with mLin-7. J.Biol.Chem. 275, 11425-11431 (2000).

60. Straight, S. W., Pieczynski, J. N., Whiteman, E. L., Liu, C. J., \& Margolis, B. Mammalian lin-7 stabilizes polarity protein complexes. J.Biol.Chem. 281, 37738-37747 (2006).

61. Wang, Q., Hurd, T. W., \& Margolis, B. Tight junction protein Par6 interacts with an evolutionarily conserved region in the amino terminus of PALS1/stardust. J.Biol.Chem. 279, 30715-30721 (2004).

62. Nam, S. C. \& Choi, K. W. Interaction of Par-6 and Crumbs complexes is essential for photoreceptor morphogenesis in Drosophila. Development 130, 4363-4372 (2003).

63. Cao, X. et al. PALS1 specifies the localization of ezrin to the apical membrane of gastric parietal cells. J.Biol.Chem. 280, 13584-13592 (2005).

64. McHugh, E. M., Zhu, W., Milgram, S., \& Mager, S. The GABA transporter GAT1 and the MAGUK protein Pals1: interaction, uptake modulation, and coexpression in the brain. Mol.Cell Neurosci. 26, 406-417 (2004).

65. Knust, E. \& Bossinger, O. Composition and formation of intercellular junctions in epithelial cells. Science 298, 1955-1959 (2002).

66. Meuleman, J., Van de Pavert, S. A., \& Wijnholds, J. Crumbs homologue 1 in polarity and blindness. Biochem.Soc.Trans. 32, 828-830 (2004).

67. van Rossum, A. G. et al. Pals1/Mpp5 is required for correct localization of Crb1 at the subapical region in polarized Muller glia cells. Hum.Mol.Genet. 15, 2659-2672 (2006).

68. Stohr, H., Stojic, J., \& Weber, B. H. Cellular localization of the MPP4 protein in the mammalian retina. Invest Ophthalmol.Vis.Sci. 44, 5067-5074 (2003).

69. Aartsen, W. M. et al. Mpp4 recruits Psd95 and Veli3 towards the photoreceptor synapse. Hum.Mol.Genet. 15, 12911302 (2006).

70. Stohr, H. et al. Membrane-associated guanylate kinase proteins MPP4 and MPP5 associate with Veli3 at distinct intercellular junctions of the neurosensory retina. J.Comp Neurol. 481, 31-41 
(2005).

71. Li, M., Zhang, S. S., \& Barnstable, C. J. Developmental and tissue expression patterns of mouse Mpp4 gene. Biochem. Biophys.Res.Commun. 307, 229-235 (2003).

72. Yang, J. et al. Mpp4 is required for proper localization of plasma membrane calcium ATPases and maintenance of calcium homeostasis at the rod photoreceptor synaptic terminals. Hum.Mol. Genet. (2007).

73. Malicki, J., Jo, H., \& Pujic, Z. Zebrafish $\mathrm{N}$-cadherin, encoded by the glass onion locus, plays an essential role in retinal patterning. Dev.Biol. 259, 95-108 (2003).

74. Malicki, J. Cell fate decisions and patterning in the vertebrate retina: the importance of timing, asymmetry, polarity and waves. Curr.Opin.Neurobiol. 14, 15-21 (2004).

75. Koike, C. et al. Function of atypical protein kinase $C$ lambda in differentiating photoreceptors is required for proper lamination of mouse retina. J.Neurosci. 25, 10290-10298 (2005).

76. Suzuki, A. et al. Atypical protein kinase $\mathrm{C}$ is involved in the evolutionarily conserved par protein complex and plays a critical role in establishing epitheliaspecific junctional structures. J.Cell Biol. 152, 1183-1196 (2001).

77. Lemmers, C. et al. CRB3 binds directly to Par6 and regulates the morphogenesis of the tight junctions in mammalian epithelial cells. Mol.Biol.Cell 15, 1324-1333 (2004).

78. Hurd, T. W., Gao, L., Roh, M. H., Macara, I. G., \& Margolis, B. Direct interaction of two polarity complexes implicated in epithelial tight junction assembly. Nat.Cell Biol. 5, 137-142 (2003).

79. Dimitratos, S. D., Woods, D. F., Stathakis, D. G., \& Bryant, P. J. Signaling pathways are focused at specialized regions of the plasma membrane by scaffolding proteins of the MAGUK family. Bioessays 21, 912-921 (1999).

80. Sakisaka, T. \& Takai, Y. Cell adhesion molecules in the CNS. J.Cell Sci. 118, 5407-5410 (2005).

81. Fukuhara, A. et al. Role of nectin in organization of tight junctions in epithelial cells. Genes Cells 7, 1059-1072 (2002).

82. Mizoguchi, A. et al. Nectin: an adhesion molecule involved in formation of synapses. J.Cell Biol. 156, 555-565 (2002).

83. Cereijido, M., Shoshani, L., \& Contreras, R. G. Molecular physiology and pathophysiology of tight junctions. I. Biogenesis of tight junctions and epithelial polarity. Am.J.Physiol Gastrointest.Liver Physiol 279, G477-G482 (2000).

84. Fanning, A. S., Mitic, L. L., \& Anderson, J. M. Transmembrane proteins in the tight junction barrier. J.Am.Soc. Nephrol. 10, 1337-1345 (1999).

85. Citi, S. \& Cordenonsi, M. Tight junction proteins. Biochim.Biophys. Acta 1448, 1-11 (1998).

86. Tang, V. W. Proteomic and bioinformatic analysis of epithelial tight junction reveals an unexpected cluster of synaptic molecules. Biol.Direct. 1, 37 (2006).

87. Pierce, J. P., Mayer, T., \& McCarthy, J. B. Evidence for a satellite secretory pathway in neuronal dendritic spines. Curr.Biol. 11, 351-355 (2001).

88. Martin, K. C., Barad, M., \& Kandel, E. R. Local protein synthesis and its role in synapse-specific plasticity. Curr.Opin.

Neurobiol. 10, 587-592 (2000).

89. Fanning, A. S. \& Anderson, J. M.

Protein modules as organizers of membrane structure. Curr.Opin.Cell Biol. 11, 432-439 (1999).

90. Funke, L., Dakoji, S., \& Bredt, D. S. Membrane-associated guanylate kinases regulate adhesion and plasticity at cell 
junctions. Annu.Rev.Biochem. 74, 219-245 (2005).

91. Krapivinsky, G., Medina, I., Krapivinsky, L., Gapon, S., \& Clapham, D. E. SynGAP-MUPP1-CaMKII synaptic complexes regulate p38 MAP kinase activity and NMDA receptor-dependent synaptic AMPA receptor potentiation. Neuron 43, 563-574 (2004).

92. Cayouette, M. \& Raff, M. The orientation of cell division influences cell-fate choice in the developing mammalian retina. Development 130, 2329-2339 (2003).

93. Chenn, A. \& McConnell, S. K.

Cleavage orientation and the asymmetric inheritance of Notch1 immunoreactivity in mammalian neurogenesis. Cell 82, 631-641 (1995).

94. Quarmby, L. M. \& Parker, J. D. Cilia and the cell cycle? J.Cell Biol. 169, 707-710 (2005).

95. Fan, S. et al. Polarity proteins control ciliogenesis via kinesin motor interactions. Curr.Biol. 14, 1451-1461 (2004).

96. Mburu, P. et al. Whirlin complexes with p55 at the stereocilia tip during hair cell development. Proc.Natl.Acad.Sci. U.S.A 103, 10973-10978 (2006).

97. Kikkawa, Y. et al. Mutant analysis reveals whirlin as a dynamic organizer in the growing hair cell stereocilium. Hum. Mol.Genet. 14, 391-400 (2005).

98. Mburu, P. et al. Defects in whirlin, a PDZ domain molecule involved in stereocilia elongation, cause deafness in the whirler mouse and families with DFNB31. Nat.Genet. 34, 421-428 (2003).

99. Nunomura, W., Takakuwa, Y., Parra, M., Conboy, J., \& Mohandas, N. Regulation of protein 4.1R, p55, and glycophorin $C$ ternary complex in human erythrocyte membrane. J.Biol.Chem. 275, 24540-24546 (2000).

100. Hagiwara, H., Ohwada, N., \& Takata, K. Cell biology of normal and abnormal ciliogenesis in the ciliated epithelium. Int.Rev.Cytol. 234, 101-141 (2004).

101. Boisvieux-Ulrich, E., Laine, M. C., \& Sandoz, D. Cytochalasin D inhibits basal body migration and ciliary elongation in quail oviduct epithelium. Cell Tissue Res. 259, 443-454 (1990).

102. Etienne-Manneville, S. \& Hall, A. Cell polarity: Par6, aPKC and cytoskeletal crosstalk. Curr.Opin.Cell Biol. 15, 67-72 (2003).

103. Shin, K., Wang, Q., \& Margolis, B. PATJ regulates directional migration of mammalian epithelial cells. EMBO Rep. 8, 158-164 (2007).

104. Djiane, A., Yogev, S., \& Mlodzik, M. The apical determinants aPKC and dPatj regulate Frizzled-dependent planar cell polarity in the Drosophila eye. Cell 121, 621-631 (2005).

105. Lu, B., Roegiers, F., Jan, L. Y., \& Jan, Y. N. Adherens junctions inhibit asymmetric division in the Drosophila epithelium. Nature 409, 522-525 (2001).

106. Giebel, B. \& Wodarz, A. Tumor suppressors: control of signaling by endocytosis. Curr.Biol. 16, R91-R92 (2006).

107. Lu, H. \& Bilder, D. Endocytic control of epithelial polarity and proliferation in Drosophila. Nat.Cell Biol. 7, 1232-1239 (2005).

108. Ivanov, A. I., Nusrat, A., \& Parkos, C. A. Endocytosis of the apical junctional complex: mechanisms and possible roles in regulation of epithelial barriers. Bioessays 27, 356-365 (2005).

109. Macara, I. G. \& Spang, A. Closing the GAP between polarity and vesicle transport. Cell 125, 419-421 (2006).

110. Michel, D. et al. PATJ connects and stabilizes apical and lateral components of tight junctions in human intestinal cells. J.Cell Sci. 118, 4049-4057 (2005). 
111. Zimmermann, P. et al. Syndecan recycling [corrected] is controlled by syntenin-PIP2 interaction and Arf6. Dev.

Cell 9, 377-388 (2005).

112. Weil, D. et al. Defective myosin

VIIA gene responsible for Usher syn-

drome type 1B. Nature 374, 60-61 (1995).

113. Gibson, F. et al. A type VII myosin encoded by the mouse deafness gene shaker-1. Nature 374, 62-64 (1995). 



\section{CHAPTER 7}

General discussion 



\section{Crumbs recruits a subcortical protein scaffold}

This thesis describes the identification of novel members of the Crumbs protein complex in the mammalian retina. The search for binding partners that directly bind to Crumbs revealed membrane palmitoylated protein 5 (MPP5) as an interacting protein for the PDZ binding motif. MPP5 contains several protein-protein interaction domains, such as a coiled-coiled region, two L27 domains, and a MAGUK module consisting of a PDZ, SH3, HOOK and GUK domain. By using yeast two-hybrid screens, family members MPP1 and MPP4 were found to be part of the protein complex (Chapter 2 and 3 ). MPP proteins can potentially form large networks by the identified homo- and heterodimerization of the $\mathrm{SH} 3$ and GUK domains. In addition, they serve as anchoring molecules for ion channels, receptors or adhesion molecules. In the retina, the analysis of putative channels, receptors and adhesion molecules that could be part of the MPP scaffold, besides Crumbs, has only just commenced.

MPP1 provides a physical connection between the Crumbs complex (via MPP5) and the Usher protein complex (via whirlin) in the retina (Chapter 3). The MPP1-whirlin interaction has also been described in hair cells in the inner ear, where whirlin is known to mediate actin polymerization and stereocilia growth ${ }^{1}$. Actin-filled stereocilia develop from microvilli on the surface of hair cells in organized rows that increase in length towards the kinocilium ${ }^{2-4}$. At a specific time point in hair cell development, whirlin and MPP1 proteins are found at similar subcellular compartments. In the whirler mouse, which lacks functional whirlin, expression of MPP1 is ablated in the stereocilia and at other sites at the apical hair cell surface from P5 onwards. This suggests that the whirlin-MPP1 interaction is very dynamic and that whirlin is required for MPP1 localization from P5 onwards until whirlin expression fades out at P14 ${ }^{5}$. In light of the interaction of MPP1 and MPP5, it is tempting to speculate that MPP5, if expressed in hair cells, may be involved in hair cell polarity.

The localization of the whirlin-MPP1-MPP5 complex during retinal development is not known. Our studies demonstrate the interaction and localization of these proteins in adult animals (older than P20), although at E14.5 and E16.5 the mRNA transcript of MPP1 was detected in the neuroblastic layer of the retina similar to whirlin. In adult animals, MPP1, MPP5 and whirlin might exert a function at the outer limiting membrane (OLM) in the establishment and/or maintenance of apico- 
basal cell polarity. In addition, whirlin and MPP1 were found at the connecting cilium and basal bodies of photoreceptors. Their functional role in this subcellular part of the photoreceptor is unclear. The involvement of whirlin in actin filament polymerization ${ }^{5,6}$, and the interaction of MPP1 with FERM domain protein EPB41 involved in anchoring membrane proteins to the actin cytoskeleton ${ }^{1,7}$, suggest a role for both proteins in actin organization and dynamics. Actin filaments are required for proper positioning of ciliary basal bodies ${ }^{8,9}$ and the presence of both molecules at these structures in photoreceptors is in line with a role in this process.

Screening of retinal cDNA libraries using the yeast two-hybrid method did not result in interactors for the FERM binding motif of CRB1. However, by testing several candidate FERM proteins for interaction with the intracellular domain of CRB1 in yeast, FERM protein EPB41L5 was found to interact with all three human Crumbs homologues (Chapter 5). Interestingly, EPB41L5 also directly binds to the HOOK domain of MPP5, forming a CRB-MPP5-EPB41L5 complex. Others have demonstrated the presence of a similar protein complex in Drosophila and zebrafish, and reported a negative regulation of EPB41L5 on apical membrane formation ${ }^{10,11}$. We did not observe an effect on apical membrane growth in our MDCK model following overexpression of EPB41L5. In a mouse model with a null allele for Epb4.115 also no effects on apical membrane size have been observed ${ }^{12}$. Overexpression of EPB41L5 in polarized MDCK cells leads to a delay in tight junction formation. Therefore, in mammals, EPB41L5 may be more important for regulation of junction formation.

As described in Chapter 6, some protein binding domains of members of the Crumbs complex have multiple binding partners. It is not known to date whether multiple binding proteins are competing for the same binding site or that interaction partners using the same binding domains are temporally and spatially separated. For example, the HOOK domain of MPP5 is found to bind both syntenin-1 and the FERM domain protein EPB41L5 (Chapter 4 and 5). For the MPP5 - syntenin-1 interaction no functional studies have been performed, but we hypothesize that this interaction provides a molecular link between E-cadherin based cell adhesion and regulation of junction formation in the retina. Whether EPB41L5 and syntenin-1 are connected to the MPP5-Crumbs complex as a single functional unit remains to be determined.

By determining the composition of the Crumbs complex as de- 
scribed in this thesis and by correlating it to current knowledge (Chapter 6), we outlined a protein interaction network that provided information regarding the function of this complex. Crumbs molecules are conserved between species and their intracellular domains are involved in organizing a macromolecular protein scaffold. In the mammalian retina, CRB1 is involved in maintenance of cell-to-cell adhesion at the outer limiting membrane (OLM). Some components of the complex are found in the photoreceptor synapses and/or at the region of the connecting cilium, besides localization apical to the OLM. Moreover, studies using polarized cell cultures or model organisms, like Drosophila and zebrafish, reveal a putative function of Crumbs in retinal patterning, ciliogenesis and vesicular transport.

\section{The role of the extracellular domain of Crumbs}

Whereas the intracellular domain of CRBs allows the build-up of a macromolecular protein complex, the function of the extracellular domain remains largely unknown (Chapter 6). Studying the function of the extracellular domain of Crumbs was not part of this project, but important advances regarding its putative function have been described by others.

Although varying in size, the extracellular domains of CRB1, CRB2 and CRB3 proteins are (predicted to be) N-glycosylated ${ }^{13,14}$. In mammals, glycosylated proteins and glycosaminoglycans are abundantly present in the space between the outer limiting membrane and the RPE, the so-called interphotoreceptor space ${ }^{15}$. The content of the interphotoreceptor space, the interphotoreceptor matrix, provides support for the aforementioned cells and ensures the integrity and function of photoreceptor cells ${ }^{16}$. The interphotoreceptor space is a barrier, interposed between the capillaries of the choroid that supply nourishment to the photoreceptors, and allows passage of vitamin A to regenerate rhodopsin in the visual cycle of rod photoreceptors ${ }^{17}$. Loss of adherence between the RPE and photoreceptor layer in e.g. retinal detachment, rapidly leads to photoreceptor death. A function for the large extracellular domains of the CRB1 and/or CRB2 proteins, and even for the smaller extracellular domain of CRB3 in this interphotoreceptor matrix could be envisioned by assuring adhesion between photoreceptors as well as between photoreceptors and Müller glial cells. Their extracellular domains could hypothetically be involved in homotypic/heterotypic interactions or in interactions with the interphotoreceptor matrix itself, providing the cor- 
rect distance between photoreceptors and Müller glial cells. When this distance is altered, e.g. by mutations in the extracellular domain of CRB1 as found in patients with retinal degeneration (see Chapter 6) or by the fact that no CRB1 protein is present as seen in the $\mathrm{Crb}^{-/}$mouse, communication between the cells could be impaired. This could ultimately account for a loss of signals that control adhesion. Recently, a large 2165amino acid secreted protein called spacemaker (SPAM) was identified in Drosophila, containing seven epidermal growth factor (EGF)-like repeats, a linker region containing multiple sites for glycosaminoglycan addition, and a carboxy terminus including four alternating repeats of EGF-like and Laminin G domains. Spacemaker is crucial in the creation of the inter-rhabdomeral space and determining its size (rhabdomeres are the functional equivalents of mammalian photoreceptor outer segments) ${ }^{18}$. The fact that the extracellular domains of CRB1 and CRB2 have a similar domain structure would support a similar function of these proteins in the interphotoreceptor matrix.

\section{Differential localization of Crumbs proteins in the retina}

Contradictory findings have been reported regarding the localization of the Crb1 protein in the retina. Using a peptide antibody against the intracellular domain, CRB1 was first detected at the region of the OLM, the Müller glia and in the inner (IS) and outer segments (OS) of cone photoreceptors ${ }^{19}$. Staining of the IS and OS of cones has not been observed by others and could be the result of a fixation artifact in the staining procedure. Furthermore, eight out of fourteen peptide residues used to raise the polyclonal antibody are identical to the Crb2 sequence, suggesting potential cross-reactivity, which makes the interpretation of this staining pattern even more complex.

Antibodies against the extracellular domain were able to detect both Crb1 isoforms (with and without the intracellular domain) at the region of the OLM, photoreceptor inner segments and apical processes of Müller glial cells and in the outer nuclear layer (ONL) ${ }^{20}$. In $r d 8$ mice that lack the transmembrane and intracellular part of Crb1, staining in the inner segments appeared the same while staining in the apical processes of Müller glial cells and OLM was diffuse or absent. Apparently, the intracellular domain is important for localization of Crb1 in Müller glia. Human and mouse CRB1/Crb1 were detected apical to the region of the OLM by immunohistochemistry using antibodies against 
their intracellular domain. This region of the OLM has been specified as the subapical region (SAR) ${ }^{21}$. The antibody raised against the mouse intracellular domain (AK2) cross-reacts with human CRB1, while the antibody against human CRB1 (AK7) was later found to be a pan-Crb antibody. No additional staining of complete inner or outer segments of photoreceptors, Müller glia apical villi or reactivity in the ONL was observed with both AK2 and AK7 antibodies. Immuno-electron microscopy (immuno-EM) with the AK2 antibody detected Crb1 mainly at the SAR in the Müller glial cells ${ }^{22}$. Using immuno-EM, Crb2 and Crb3 were detected at the SAR in both photoreceptors and Müller glia ${ }^{22}$.

Despite some inconsistency in CRB1/Crb1 stainings in the retina, overlap can be found at the SAR where both photoreceptors and Müller glial cells reside. At the SAR, CRB2 and CRB3 were detected in Müller glia and photoreceptors. The CRB1 isoform that contains the intracellular domain and transmembrane region appears to be mainly found in the Müller glia, while the isoform without the intracellular domain could be the main isoform that is present in photoreceptors based on data of the $r d 8$ mouse. To shed more light on this matter, antibodies should be raised specifically against the latter variant that has a unique carboxy terminus of 40 amino acids without a transmembrane or intracellular domain.

\section{Disease-causing mutations and the Crumbs complex}

Thus far, of all members of the Crumbs complex, only mutations in the CRB1 gene have been found to cause retinal dystrophies. Assuming that changes in genes/proteins in the same pathway or protein network could be implicated in causing a similar disease, several members of the Crumbs protein complex were analyzed for mutations (unpublished data).

The open reading frame and splice junctions of $C R B 2{ }^{23}$ and $C R B 3$ were analyzed for mutations by single-strand conformation analysis and direct nucleotide sequencing in 79 LCA and 85 autosomal recessive or isolated RP patients, but no causative mutations were identified. Mutation analysis for MPP5 in 81 autosomal recessive or isolated cone-rod dystrophy (CRD) patients, 79 LCA patients, and 85 autosomal recessive or isolated RP patients using conformation-sensitive capillary electrophoresis, and additional sequence analysis of 4 patients with RP with para-arteriolar preservation of the RPE and 6 patients with RP/Coats also did not reveal any causative mutations. Similarly, mutation analysis of the entire 
open reading frame of the MPP4 gene in an autosomal recessive RP family of which the phenotype cosegregated with a region on chromosome 2 containing the MPP4 gene ${ }^{24}$, as well as a panel of 300 unrelated patients with retinitis pigmentosa ${ }^{25}$ revealed no pathologic mutations. In addition, we did not identify any MPP4 mutations in a panel of 58 patients with LCA. Four LCA patients showed homozygosity for a chromosomal region at 2q14.2 (identified by whole-genome linkage analysis using SNP arrays) containing EPB41L5 (A.I. den Hollander, personal communication). Sequence analysis of the 24 protein coding exons however did not reveal any disease-associated mutations.

Several explanations can be given for the absence of disease-causing mutations in genes encoding Crumbs homologues and interacting proteins. Mutations in Crumbs complex members may cause completely different non-ocular phenotypes, or even may be embryonically lethal. CRB2, based on its similarity to Drosophila Crumbs, is the ancestral CRB gene and is expressed in many tissues. Mutations in CRB2 might not be tolerated or could lead to a complex phenotype involving several defects. This holds true for other components of the Crumbs protein complex as well. MPP1, EPB41L5 and MPP5 are expressed in the developing embryo, not only in the retina, but also in many other organs. The MPP1 protein exerts an important function in the inner ear, while expression of the MPP1 gene is seen in developing bone structures and the cranial nerve ganglia among others. Mutations in MPP5 could be embryonically lethal, since gene expression was detected already in E10.5 embryos in the developing neural tube and optic vesicle, as well as in the branchial arches and kidney. EPB41L 5 expression is detected at the same developmental stage in the same tissues as MPP5 and mutations in EPB41L 5 lead to early developmental defects in mice ${ }^{12,26}$.

Alternatively, mutations in Crumbs complex members could give rise to ocular defects other than photoreceptor cell death. MPP4 is predominantly expressed in the retina, but it is mainly found in the photoreceptor synapse and was recently implicated in destabilization of the presynaptic protein complex. Furthermore, in $\mathrm{MPP}^{-/}$mice, a disturbed calcium homeostasis and synaptic transmission were observed, but no retinal degeneration ${ }^{27,28}$.

Studies from zebrafish show that multiple components are involved in retinal patterning defects, such as Crb2a, EPB41L5/YMO1, MPP5/nok and aPKC. Patterning defects in humans might be present, e.g. in LCA patients, but whether or not this phenotype is similar to what 
has been found in zebrafish is difficult to assess, since no histological studies have been performed on human tissue. In addition, in zebrafish additional Crumbs variants are present due to whole genome duplication. Zebrafish Crb1 is the homologue of mammalian CRB1 based on the amino acid sequence, while zebrafish $\mathrm{Crb} 2 \mathrm{~b}$ is probably the functional homologue of CRB1. Zebrafish Crb2a could represent the sequential and functional homologue of mammalian CRB2, hampering straightforward interspecies comparison.

On the contrary, functional redundancy in Crumbs protein complex members could also result in the absence of a clinical manifestation. However, for EPB41L5 and MPP4 it is not likely that their function can be taken over by EPB41 or MPP protein family members respectively, since knock-out mice do show defects.

Additional explanations secondary to the previous ones are linked to the undertaken approach to analyze mutations in certain patient groups. The number of patients per clinical subgroup that were analyzed might have been too low. Many retinal dystrophy genes are only mutated in $1-2 \%$ of all patients. The group size would have to be considerably increased to statistically raise the detection chance. Second, mainly DNA of patients with autosomal recessive RP was analyzed. It is possible that some of these genes might be implicated in autosomal dominant retinal dystrophies. Third, only the coding sequence and intron-exon boundaries are tested, while changes in introns, in the promotor region or regulatory elements could be causative for a disease. Mutation analysis on mRNA may reveal any of these changes. Fourth, it is possible that mutations in CRB2 or CRB3 and interacting proteins are associated with LCA, RP, or retinal dystrophies with additional clinical symptoms. Patients groups with multiple clinical features were not analyzed.

\section{Crumbs complex components as modifier genes}

The presence of modifier genes, besides environmental factors, have been proposed to influence the phenotypic outcome of the $r d 8$ mouse, which was observed to depend on the background strain ${ }^{20}$. Modifier effects might explain the phenotypic variability that was found in RP and LCA patients as well. The majority of heterozygous carriers of LCA-associated mutations, including CRB1 mutations, can have a mild, but clearly detectable retinal dysfunction ${ }^{29,30}$. Furthermore, the fact that two RP patients with null mutations do not suffer from blindness from birth indicates that complete loss of CRB1 function is not enough to cause LCA, and 
leaves an important possibility for influence by modifier genes ${ }^{31}$. It could be hypothesized that some changes in members of the Crumbs complex are modifiers for retinal degenerations. A subtle change in a component of the Crumbs pathway, not enough to cause a disease by itself, could very well alter the threshold of disease onset due to a mutation in a functionally related gene, or alter the disease severity.

\section{Future directions}

To gain more insight into the function of Crumbs and the interacting proteins, more efforts could be undertaken to define the complex more specifically. For example, determining the composition of the protein complex at specific developmental stages, while specifying its function in different cell types will give important answers to questions on how the Crumbs complex is spatially and temporally regulated. By using specific antibodies for retinal stainings at different developmental stages, this information could be obtained. Furthermore, proteomic approaches such as GST pull down assays, immunoprecipitations or tandem-affinity purification (TAP) techniques followed by mass-spectrometry detection, may reveal more components of the associated protein complex in a single experiment. Some attempts have been undertaken to purify additional components of the Crumbs complex by GST-pull down analysis with the intracellular domain of CRB1 in bovine retinas. Pull down samples using GST-CRB1 $1^{\text {INTRA }}$ or GST alone were compared on a 2D-DIGE-gel system and differential spots were cut out and analyzed by mass-spectrometry. This experiment did not yield any novel interacting proteins. For a follow-up experiment, different extraction buffers could be used to make retinal lysates, or immunoprecipitations from the same tissue could be performed. The TAP technique requires a double tagged construct and therefore depends upon the use of transfected cultured cells, e.g. retinal cells or even a polarized cell type such as MDCK cells (Gloeckner et al., Proteomics, in press).

To increase our understanding of the function of Crumbs molecules and to ascertain why mutations in $C R B 1$ cause retinal degeneration, it will be essential to determine the retinal cell type in which Crumbs exerts its function. The nature of the complex in Müller glial cells versus photoreceptor could be established by aforementioned techniques, making use of separate preparations of photoreceptor and Müller glial cell cultures from e.g. bovine or porcine retinas ${ }^{32,33}$. The CRB1 isoform that 
contains the intracellular domain and transmembrane region could be the main isoform in Müller glia, while the isoform without the intracellular domain might be present only in photoreceptors or both. CRB2 and CRB3 were found both in photoreceptors and Müller glial cells. The presence of different CRB1 isoforms, as well as the complementary nature of the intracellular domains of CRB1, CRB2 and CRB3 proteins in providing a scaffold that allows the build-up of an intracellular protein complex, needs to be taken into account. By using (isoform) specific Crumbs antibodies to precipitate the attached complex, its composition could be resolved.

The possibility of homo/heterotypic interactions between the extracellular domains of CRB1, CRB2 and maybe even CRB3, could be examined. It is not known if CRB1 molecules can bind to each other to form a functional unit, either indirectly via their intracellular components or maybe even via their extracellular domains, and how this might lead to the build-up of a macromolecular complex. In this respect, determining the stoichiometry of the complex will also be important to learn more about the function. We have seen that MPP proteins could potentially form large networks by binding to family members. In addition, they serve as anchoring molecules for ion channels, receptors or adhesion molecules. Defining the number of MPP proteins that are needed to form a functional unit, is key to understanding the regulation of the Crumbs complex.

Omitting components one by one from the complex by performing siRNA on retinal explants may provide crucial information on which protein is essential for the build-up of the complex. Efforts for MPP5 in this direction have been proven successful ${ }^{22}$ and could be expanded for other Crumbs complex members. In this way, the putative redundancy of CRB and MPP homologues could be determined. In addition, creating conditional knockout mice for components that are crucial in embryonic development and otherwise would lead to an embryonic lethal phenotype may be helpful to establish the function of these intriguing proteins. 


\section{REFERENCES}

1. Mburu, P. et al. Whirlin complexes with p55 at the stereocilia tip during hair cell development. Proc.Natl.Acad.Sci. U.S.A 103, 10973-10978 (2006).

2. Forge, A., Souter, M., \& DenmanJohnson, K. Structural development of sensory cells in the ear. Semin.Cell Dev. Biol. 8, 225-237 (1997).

3. Tilney, L. G., Tilney, M. S., \& DeRosier, D. J. Actin filaments, stereocilia, and hair cells: how cells count and measure. Annu.Rev.Cell Biol. 8, 257-274 (1992).

4. Denman-Johnson, K. \& Forge, A. Establishment of hair bundle polarity and orientation in the developing vestibular system of the mouse. J.Neurocytol. 28, 821-835 (1999).

5. Kikkawa, Y. et al. Mutant analysis reveals whirlin as a dynamic organizer in the growing hair cell stereocilium. Hum. Mol.Genet. 14, 391-400 (2005).

6. Mburu, P. et al. Defects in whirlin, a PDZ domain molecule involved in stereocilia elongation, cause deafness in the whirler mouse and families with DFNB31. Nat.Genet. 34, 421-428 (2003).

7. Nunomura, W., Takakuwa, Y., Parra, M., Conboy, J., \& Mohandas, N. Regulation of protein 4.1R, p55, and glycophorin $\mathrm{C}$ ternary complex in human erythrocyte membrane. J.Biol.Chem. 275, 24540-24546 (2000).

8. Hagiwara, H., Ohwada, N., \& Takata, K. Cell biology of normal and abnormal ciliogenesis in the ciliated epithelium. Int.Rev.Cytol. 234, 101-141 (2004).

9. Boisvieux-Ulrich, E., Laine, M. C., \& Sandoz, D. Cytochalasin D inhibits basal body migration and ciliary elongation in quail oviduct epithelium. Cell Tissue Res. 259, 443-454 (1990).

10. Laprise, P. et al. The FERM protein yurt is a negative regulatory component of the Crumbs complex that controls epithelial polarity and apical membrane size. Dev.Cell 11, 363-374 (2006).

11. Hsu, Y. C., Willoughby, J. J., Christensen, A. K., \& Jensen, A. M. Mosaic Eyes is a novel component of the Crumbs complex and negatively regulates photoreceptor apical size. Development 133, 4849-4859 (2006).

12. Lee, J. D., Silva-Gagliardi, N. F., Tepass, U., McGlade, C. J., \& Anderson, $\mathrm{K}$. V. The FERM protein Epb4.115 is required for organization of the neural plate and for the epithelial-mesenchymal transition at the primitive streak of the mouse embryo. Development 134, 2007 2016 (2007).

13. Makarova, O., Roh, M. H., Liu, C. J., Laurinec, S., \& Margolis, B. Mammalian Crumbs 3 is a small transmembrane protein linked to protein associated with Lin-7 (Pals1). Gene 302, 21-29 (2003).

14. Kantardzhieva, A. et al. MPP5 recruits MPP4 to the CRB1 complex in photoreceptors. Invest.Ophthalmol.V is. Sci. 46, 2192-2201 (2005).

15. Berman, E. R. \& Bach, G. The acid mucopolysaccharides of cattle retina. Biochem.J. 108, 75-88 (1968).

16. Feeney, L. The interphotoreceptor space. I. Postnatal ontogeny in mice and rats. Dev.Biol. 32, 101-114 (1973).

17. Dowling, J. E. Chemistry of visual adaptation in the rat. Nature 188, 114-118 (1960). 
18. Zelhof, A. C., Hardy, R. W., Becker, A., \& Zuker, C. S. Transforming the architecture of compound eyes. Nature 443, 696-699 (2006).

19. Pellikka, M. et al. Crumbs, the Drosophila homologue of human CRB1/RP12, is essential for photoreceptor morphogenesis. Nature 416, 143-149 (2002).

20. Mehalow, A. K. et al. CRB1 is essential for external limiting membrane integrity and photoreceptor morphogenesis in the mammalian retina. Hum.Mol. Genet. 12, 2179-2189 (2003).

21. Van de Pavert, S. A. et al. Crumbs homologue 1 is required for maintenance of photoreceptor cell polarization and adhesion during light exposure. J.of Cell Sci. 117, 4169-4177 (2004).

22. van Rossum, A. G. et al. Pals1/Mpp5 is required for correct localization of Crb1 at the subapical region in polarized Muller glia cells. Hum.Mol.Genet. 15, 2659-2672 (2006).

23. van den Hurk, J. A. J. M. et al. Characterization of the Crumbs homolog 2 (CRB2) gene and analysis of its role in retinitis pigmentosa and Leber congenital amaurosis. Mol.Vis. 11, 263-273 (2005).

24. Tuson, M., Marfany, G., \& GonzalezDuarte, R. Mutation of CERKL, a novel human ceramide kinase gene, causes autosomal recessive retinitis pigmentosa (RP26). Am.J.Hum.Genet. 74, 128-138 (2004).

25. Conte, I. et al. Characterization of MPP4, a gene highly expressed in photoreceptor cells, and mutation analysis in retinitis pigmentosa. Gene 297, 33-38 (2002).
26. Garcia-Garcia, M. J. et al. Analysis of mouse embryonic patterning and morphogenesis by forward genetics. Proc.Natl.Acad.Sci.U.S.A 102, 5913-5919 (2005).

27. Aartsen, W. M. et al. Mpp4 recruits Psd95 and Veli3 towards the photoreceptor synapse. Hum.Mol.Genet. 15, 12911302 (2006).

28. Yang, J. et al. Mpp4 is required for proper localization of plasma membrane calcium ATPases and maintenance of calcium homeostasis at the rod photoreceptor synaptic terminals. Hum.Mol. Genet. 16, 1017-1029 (2007).

29. Yzer, S. et al. CRB1 heterozygotes with regional retinal dysfunction: implications for genetic testing of leber congenital amaurosis. Invest.Ophthalmol. Vis.Sci. 47, 3736-3744 (2006).

30. Galvin, J. A., Fishman, G. A., Stone, E. M., \& Koenekoop, R. K. Clinical phenotypes in carriers of Leber congenital amaurosis mutations. Ophthalmology 112, 349-356 (2005)

31. den Hollander, A. I. et al. CRB1 mutation spectrum in inherited retinal dystrophies. Hum.Mutat. 24, 355-369 (2004).

32. Hauck, S. M., Suppmann, S., \& Ueffing, M. Proteomic profiling of primary retinal Muller glia cells reveals a shift in expression patterns upon adaptation to in vitro conditions. Glia 44, 251-263 (2003).

33. Hicks, D., Forster, V., Dreyfus, H., $\&$ Sahel, J. Survival and regeneration of adult human photoreceptors in vitro. Brain Res. 643, 302-305 (1994). 

SUMMARY 

Mutations in the Crumbs homologue 1 (CRB1) gene are causative for Leber congenital amaurosis (LCA), a congenital or early-onset blindness, as well as for several subtypes of retinitis pigmentosa (RP). In RP, rod photoreceptors are first affected, leading to night blindness, followed by progressive death of cone photoreceptors in the central macula affecting daytime vision. This restricts the visual field initially in the midperiphery and at later stages can lead to complete loss of vision.

The CRB1 gene encodes a transmembrane protein that is implicated in mechanisms that control cell polarity. The shape of cells, the oriented alignment of the cytoskeleton and the uneven distribution of organelles and molecules all contribute to cell polarity. A polarized phenotype of a cell reflects its ability to establish functional and separate membrane domains that allow for both structural and signaling functions. Crumbs is involved in maintaining cell polarity and tissue integrity by defining the apical membrane domain in epithelial cells as well as in photoreceptors in different organisms. In the mammalian retina the core Crumbs complex localizes apical to the outer limiting membrane of the retina, the so-called subapical region (SAR) where photoreceptors and Müller glial cells contact each other.

The 37 amino acids of the Crumbs protein that are located inside the cell, form an attachment point for an intracellular protein complex that is essential for cell polarity. In this thesis, the composition of the Crumbs protein network that is associated with the intracellular domain is studied. Identification of novel components of the intracellular Crumbs complex will lead to a more profound understanding of its function in different cellular processes in health and disease. In addition, defects in components of the Crumbs protein complex might be involved in the pathogenesis of inherited retinal disorders (Chapter 1).

Yeast two-hybrid screens of bovine and human retinal cDNA libraries were performed with the intracellular domain of Crumbs homologue 1 (CRB1) to identify novel interaction partners. This small 37-amino acid domain contains two conserved binding motifs, namely a PDZ and a FERM binding motif. The membrane-associated guanylate kinase protein (MAGUK) protein MPP5 (also known as PALS1) binds to the PDZ binding motif of CRB1 (Chapter 2). MPP5 consists of several protein binding domains and further analysis of binding partners for MPP5 by yeast two-hybrid screening led to the identification of MPP family members MPP1 and MPP4 (Chapter 2 and 3). These membrane palmitoylated proteins have largely expanded the Crumbs protein network 
and their binding properties provide an intricate mechanism to build and regulate large networks comprised of similar building blocks.

MPP1 was found to bind to whirlin, a key regulator of the Usher protein network that is implicated in actin polymerization. Mutations in DFNB31 encoding the whirlin protein are found in cases of isolated deafness and in patients with Usher syndrome (sensorineural deafness and retinitis pigmentosa). MPP1 provides a link between the Usher protein network via whirlin and the Crumbs protein complex via MPP5 in the retina. These findings might point to a shared function of these protein complexes in actin polymerization and cell polarity establishment or maintenance. Besides co-localization of MPP1, MPP5 and whirlin at the SAR, MPP1 and whirlin were also found at the connecting cilium region and basal body structure (Chapter 3).

Syntenin-1 is present in the Crumbs protein complex via interaction with MPP5 at the subapical region (SAR) in the retina. Syntenin-1 has a variety of proposed functions in different polarized cell types, ranging from a role in cytoskeletal-membrane organization, protein trafficking and cell adhesion to the activation of transcription factors. A role for Syntenin-1 in the retina has not yet been described, but the association with polarity determinant MPP5 at the subapical region found here, suggests a role in retinal cell polarity processes (Chapter 4).

Three Crumbs homologues, CRB1, CRB2 and CRB3, exist in mammals. All are able to bind to MPP5 and EPB41L5, through their conserved PDZ and FERM binding motif, respectively. Conservation of the Crumbs-EPB41L5-MPP5 complex in zebrafish and Drosophila demonstrates an evolutionary importance, although its function may vary between species. In contrast to a negative effect on apical membrane expansion as identified in Drosophila and zebrafish, overexpression of EPB41L5 in polarized MDCK cells leads to a delay in tight junction formation (Chapter 5).

Some components of the Crumbs complex that were identified were subjected to mutation analysis, but no causative mutations for a retinal disease were found. This could implicate that the number of patients per (sub)clinical group that was analyzed was statistically not sufficient. Alternatively, mutations in Crumbs complex members may also cause (sub)clinical phenotypes different from a progressive photoreceptor cell death, e.g. (eye) defects of developmental origin, or syndromic phenotypes that include a retinal dystrophy, or may be embryonic lethal (Chapter 7). 
The intracellular Crumbs protein complex has been largely expanded by this work. The identified components point towards functions of the Crumbs protein complex in several cellular processes, ranging from cell adhesion to retinal patterning. Some Crumbs orthologues as well as interactors of Crumbs have been implicated in basal body and cilia related processes. It is tempting to speculate that mammalian Crumbs proteins influence ciliogenesis, asymmetric cell division and/or migration, since associations with these important processes have been reported. Besides a role in cell-cell adhesion at the region of the OLM, some components of the Crumbs complex are recently found to be involved in vesicular transport and regulation of cell polarity by influencing endocytosis (Chapter 6). 



\section{SAMENVATTING}



Mutaties in het Crumbs homoloog 1 (CRB1) gen veroorzaken verschillende vormen van erfelijke blindheid, waaronder Leber congenitale amaurosis (LCA) en retinitis pigmentosa (RP). LCA wordt gekenmerkt door aangeboren blindheid of verlies van gezichtsvermogen op zeer jonge leeftijd. Patiënten met RP hebben in eerste instantie last van nachtblindheid door het afsterven van lichtgevoelige staafjes in het netvlies. Op latere leeftijd sterven ook de kegeltjes af, waardoor uiteindelijk totale blindheid kan ontstaan.

Het $C R B 1$ gen codeert voor een eiwit dat door het membraan van cellen heen steekt. Daarbij bevindt het grootste gedeelte van dit transmembraaneiwit zich buiten de cel en bevindt zich een klein gedeelte van 37 aminozuren aan de binnenzijde. Het CRB1 eiwit is verantwoordelijk voor de regulatie van celpolariteit. Celpolariteit wordt gekenmerkt door het vermogen van een cel om het apicale en basolaterale membraan van elkaar te onderscheiden en als het ware een "bovenkant" en een "onderkant" te hebben. Hierdoor treedt er een ordening op van celorganellen, het cytoskelet en andere moleculen binnen de cel die belangrijk zijn voor de structuur en de functie van de cel. Crumbs definieert het apicale membraan van zowel epitheel cellen als fotoreceptoren in verschillende organismen. In het netvlies van zoogdieren bevindt het Crumbs eiwit zich aan de apicale zijde van de buitenste "limiting" (afgrenzende) membraan in het netvlies, de zogenaamde subapicale regio. Het buitenste 'limiting' membraan is geen echt membraan, maar lijkt daar slechts op onder de microscoop. Het is de plaats waar fotoreceptoren in nauw contact staan met de omliggende Müller glia cellen. Het Crumbs eiwit is verantwoordelijk voor celadhesie tussen de fotoreceptoren en Müller glia cellen.

De 37 aminozuren van het Crumbs eiwit die zich aan de binnenzijde van de cel bevinden, vormen een aanhechtingspunt voor een intracellulair eiwit complex dat essentieel is voor celpolariteit. In dit proefschrift wordt de compositie van het intracellulaire eiwit complex nader bestudeerd. Door nieuwe bestanddelen van dit netwerk te identificeren, zullen we meer te weten komen over de manier waarop het Crumbs eiwit zijn functie uitvoert, zowel in een gezond netvlies als mogelijk ook in netvliezen van patiënten die lijden aan erfelijke blindheid veroorzaakt door mutaties in het CRB1 gen.

Met behulp van het "yeast two-hybrid" systeem zijn banken gescreend met daarin cDNA's uit humane en rundernetvliezen. Met deze techniek kunnen eiwitten die aan het intracellulaire domein van Crumbs binden gedetecteerd worden. Het reeds bekende eiwit MPP5 (membraan 
gepalmitoyleerd eiwit 5) bleek te binden aan PDZ bindingsmotief van Crumbs worden geïdentificeerd (Hoofdstuk 2). MPP5 op zijn beurt bestaat uit een aantal bindingsmotieven waar ook andere eiwitten aan kunnen binden. Door de yeast two-hybrid screens uit breiden voor MPP5, zijn de bindingspartners en familieleden MPP1 en MPP4 gevonden (Hoofdstuk 2 en 3). Via een speciaal bindingsmechanisme zijn deze eiwitten in staat om grote netwerken te vormen en te reguleren.

Het MPP1 eiwit bindt aan whirlin, een eiwit dat gecodeerd wordt door het DFNB31 gen. Mutaties in dit gen kunnen leiden tot erfelijke doofheid of Usher syndroom (erfelijke doofblindheid). Whirlin reguleert de vorming van een Usher eiwit netwerk en is verantwoordelijk voor actine polymerisatie. MPP1 vormt een link tussen het Usher en het Crumbs eiwit netwerk, waardoor beide eiwitcomplexen betrokken kunnen zijn bij actine polymerisatie en het ontstaan en behoud van celpolariteit. Naast het feit dat MPP1, MPP5 en whirlin te vinden zijn in de subapicale regio, hebben MPP1 en whirlin mogelijk ook een functie in het connecting cilium en de "basal bodies" van fotoreceptoren (Hoofdstuk 3).

Syntenin-1 is aanwezig in het Crumbs eiwit complex via interactie met het zogenaamde "HOOK" domein van MPP5. Ook Syntenin-1 is te vinden in de subapicale regio in het netvlies. Van Syntenin-1 zijn verschillende functies gepostuleerd in gepolariseerde cellen, zoals en rol in cytoskelet-membraan ordening, eiwit transport, cel adhesie en het activeren van transcriptiefactoren. Een functie voor het syntenin-1 eiwit in het oog is niet eerder beschreven. Het feit dat syntenin-1 aan het MPP5 bindt, maakt dat het een belangrijk eiwit zou kunnen zijn voor celpolariteit (Hoofdstuk 4).

Er bestaan drie homologe Crumbs eiwitten in zoogdieren, CRB1, CRB2 en CRB3. In hoofdstuk 5 wordt beschreven dat alle drie de eiwitten zowel aan MPP5 kunnen binden als aan EPB41L5 met respectievelijk het PDZ- en het FERM bindingsmotief van het intracellulaire domein. Dit eiwitcomplex is ook teruggevonden in de fruitvlieg en zebravis hetgeen op een evolutionair belang van de binding duidt. De functie van dit complex verschilt waarschijnlijk in de verschillende organismen, omdat een effect op de expansie van apicale membranen alleen wordt gevonden in de fruitvlieg en zebravis. Bij overexpressie van het zoogdieren EB41L5 eiwit in een celmodel wordt een vertragend effect gevonden op de vorming van "tight junctions".

Sommige componenten van het Crumbs complex die hier geidentificeerd zijn, zijn onderworpen aan mutatie analyse bij patiënten met 
erfelijke oogziekten. Er zijn geen defecten gevonden die een oogziekte zouden kunnen veroorzaken. Dit zou verband kunnen houden met de relatief kleine (sub)groep van patiënten met erfelijke blindheid die zijn uitgekozen voor de analyse. Het is ook mogelijk dat defecten in deze componenten geen effect hebben op het afsterven van fotoreceptoren, maar wel een andere aandoening kunnen veroorzaken. De vroege expressie van de componenten in meerdere organen duidt erop dat deze eiwitten waarschijnlijk erg belangrijk zijn tijdens de ontwikkeling van een embryo. Defecten in deze eiwitten zijn mogelijk niet verenigbaar met het menselijk leven (Hoofdstuk 7).

In hoofdstuk 6 wordt een overzicht gegeven van hetgeen er momenteel bekend is over het Crumbs eiwitcomplex. Het intracellulaire Crumbs eiwitnetwerk is flink uitgebreid door de studies beschreven in dit proefschrift. Het lijkt erop dat het Crumbs eiwit complex betrokken is bij veschillende cellulaire processen. Sommige orthologe Crumbs moleculen alsmede Crumbs bindingspartners zijn betrokken bij processen die in de "basal bodies" en cilia plaatsvinden. Het is nog te vroeg om te kunnen zeggen dat het Crumbs complex in zoogdieren ook betrokken is bij ciliogenese, asymmetrische celdeling/celmigratie, maar de gevonden associaties in andere organismen zijn op zijn minst opmerkelijk. Naast een rol in het behoud van celadhesie op de plaats waar fotoreceptoren en Müller glia cellen elkaar raken, zijn er aanwijzingen voor een mogelijk rol van het Crumbs complex in het transport en het opnemen van celcomponenten in blaasjes. 



\section{LIST OF PUBLICATIONS}





\section{List of publications}

I.Gosens, A.I. den Hollander, F.P.M. Cremers, R.Roepman (2007) Composition and function of the Crumbs protein complex in the mammalian retina. Review. Provisionally accepted for publication in Experimental Eye Research.

I.Gosens, S.J.F. Letteboer, M.C. Peeters, F.P.M. Cremers, R. Roepman (2007) Syntenin-1 is a novel binding partner of cell-polarity determinant MPP5 in the mammalian retina. Submitted.

I. Gosens, A. Sessa, A.I. den Hollander, S.J.F. Letteboer, V. Belloni, M.L. Arends, A. Le Bivic, F.P.M. Cremers, V. Broccoli and R. Roepman (2007). FERM protein EPB41L5 is a novel member of the mammalian CRB-MPP5 polarity complex and is involved in tight junction formation.

Experimental Cell Research. Nov 15;313(19):3959-3970.

I. Gosens, E. van Wijk, F.F.J. Kersten, E. Krieger, B. van der Zwaag, T. Märker, S.J.F. Letteboer, S. Dusseljee, T. Peters, H.A. Spierenburg, I.M. Punte, U. Wolfrum, F.P.M. Cremers, H. Kremer*, and R. Roepman* (2007). MPP1/p55 links the Usher protein network and the Crumbs protein complex in the retina.

Human Molecular Genetics. Aug 15;16(16):1993-2003.

* Both senior authors contributed equally to this work

A.I. den Hollander, R.. Koenekoop, M.D. Mohamed, H.H. Arts, K. Boldt, K.V. Towns, M. Beer, M. McKibbin, S. Dharmaraj, I. Lopez, T.M. Strom, L. Ivings, G.A. Williams, K. Springell, G. Woods, H. Jafri, Y. Rashid, B. van der Zwaag, I. Gosens, F.F.J. Kersten, E. van Wijk, J.A. Veltman, M.N. Zonneveld, S.E.C. van Beersum, M.E. Cheetham, I.H. Maumenee, M. Ueffing, F.P.M. Cremers, C.F. Inglehearn, R. Roepman (2007). Mutations in lebercilin, a novel ciliary protein, cause Leber congenital amaurosis.

Nature. Genetics. Jul; 39(7):889-95.

I. Gosens*, A. Kantardzhieva*, S. Alexeeva, I.M. Punte, I. Versteeg, E. Krieger, C.A. Neefjes-Mol, A.I. den Hollander, S.J.F. Letteboer, J. Klooster, F.P.M. Cremers, R. Roepman and J. Wijnholds (2005). MPP5 recruits MPP4 to the CRB1 complex in photoreceptors.

Investigative Ophthalmology \& Visual Science. Jun; 46(6):2192-201.

* Both authors contributed equally to this work 



\section{CURRICULUM VITAE}





\section{Curriculum Vitae}

Ilse Gosens werd geboren op 6 mei 1979 te Schiedam. Haar VWO opleiding volgde zij aan het Stedelijk Gymnasium in Schiedam, waar zij in 1997 haar eindexamen behaalde. Vervolgens studeerde zij Bio-Farmaceutische Wetenschappen aan de Universiteit Leiden. Tijdens deze studie deed zij haar stage bij de vakgroep Medische Farmacologie in Leiden, waar zij onderzoek deed naar het opzetten van een celmodel om bepaalde cellulaire processen te bestuderen na dopamine receptor stimulatie. Dit onderzoek werd gedurende 1 jaar uitgevoerd onder begeleiding van Dr. Erno Vreugdenhil. Vervolgens heeft zij een tweede stage van 5 maanden gedaan aan het INSERM instituut in Bordeaux (Frankrijk) onder begeleiding van Prof. Piervi Piazza en Dr. Françoise Rouge-Pont. Het onderwerp betrof het karakteriseren van de stressrespons in verschillende muizenstammen na restraint stress. Ook heeft zij gedurende een paar maanden als documentalist gewerkt bij het Europees Octrooi bureau op de afdeling tweede toepassing van geneesmiddelen. Na haar afstuderen, begon zij op 1 september 2002 bij de afdeling Antropogenetica van het Radboud University Nijmegen Medical Centre aan het in dit proefschrift beschreven promotieonderzoek, onder leiding van Prof. Frans Cremers en Dr. Ronald Roepman. Vanaf 1 juli 2007 is zij werkzaam als toxicoloog bij het RIVM te Bilthoven waar zij onderzoek doet naar de nadelige gezondsheidseffecten van luchtverontreiniging. 

DANKWOORD 



\section{Dankwoord}

Aan het eind van de rit zou ik graag iedereen bedanken die bijgedragen heeft aan de totstandkoming van dit proefschrift.

Ik wil graag alle collega's van de sectie Moleculaire Genetica bedanken voor de gezelligheid en het warme nest waarin ik terecht kwam.

In het bijzonder, Stef en Erwin, ik ben blij dat jullie er altijd waren voor raad en daad en mij tot slot bij willen staan op de dag zelf.

Natuurlijk wil ik ook de rest van de afdeling Antropogenetica, alsmede collega's uit "de NCMLS toren", niet vergeten te bedanken voor hun wetenschappelijke bijdrage, een luisterend oor, een praatje, of hulp als ik weer eens iets nodig had om een experiment te kunnen doen.

Natuurlijk was dit proefschrift niet tot stand gekomen zonder de hulp van promotor en co-promotor. Frans, bedankt dat je me de kans gaf om op een congres mensen van wie het gezichtvermogen is aangetast te ontmoeten. Ik heb het erg gewaardeerd en het gaf mij nieuwe energie om door te gaan met het onderzoek. Ronald, jouw niet aflatende enthousiasme en vertrouwen op een goede afloop heeft zeker geholpen om het proefschrift af te ronden.

Miriam en Ingrid, ik hoop dat jullie net zoveel van de stage hebben geleerd als ik van jullie.

To all colleagues of the Crumbs consortium, thanks for your helpful discussions and for sharing your research expertise.

To all foreign colleagues, thanks for your scientific contributions to help conceive several of the chapters in this thesis.

Nieuwe collega's van het RIVM, bedankt voor jullie flexibele houding zodat het boekje voltooid kon worden.

Lieve familie en vrienden bedankt voor de broodnodige ontspanning en nu het boekje af is heb ik eindelijk meer tijd om met jullie af te spreken.

En Alwin, het was gezellig om samen thuis aan de boekjes te schrijven, maar ik ben toch erg blij dat het nu af is.

Een bijlage op dit dankwoord is te vinden op de volgende pagina. 


\section{Bijlage dankwoord}

Ik zou graag eenieder bedanken

Die me bij heeft gestaan in de flanken

Want zonder de hulp

Was dit boekje pulp

$\mathrm{Nu}$ besluit ik met vrolijke klanken

Men raadt wel, dit woord is geschreven

In een maand die we creatief beleven

Vouw deze kaart

En bewaar hem tot maart

Beschouw hem als persoonlijk gegeven
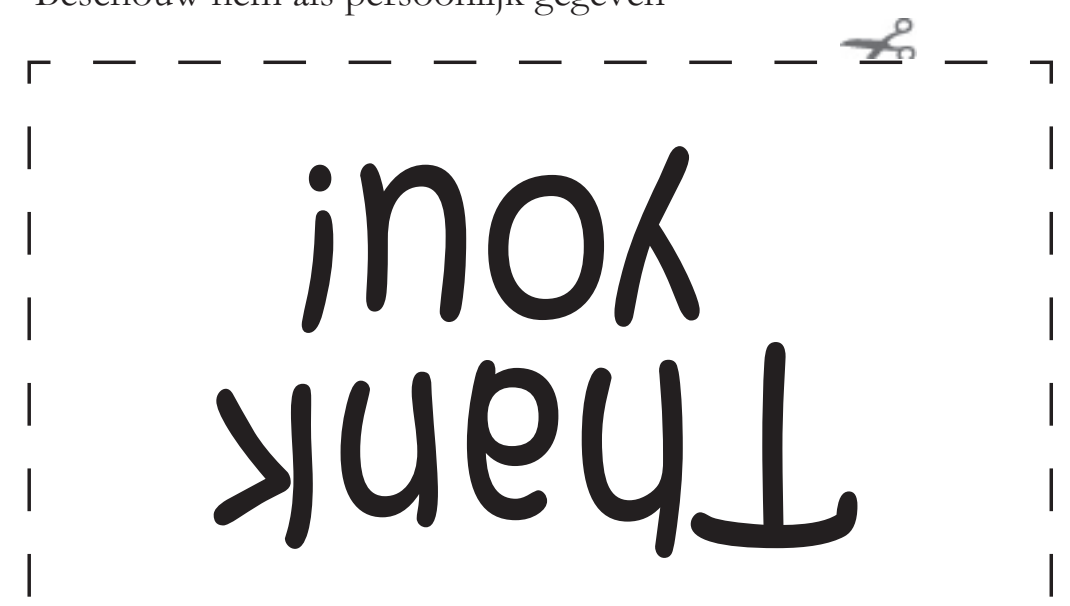

hier vouwen
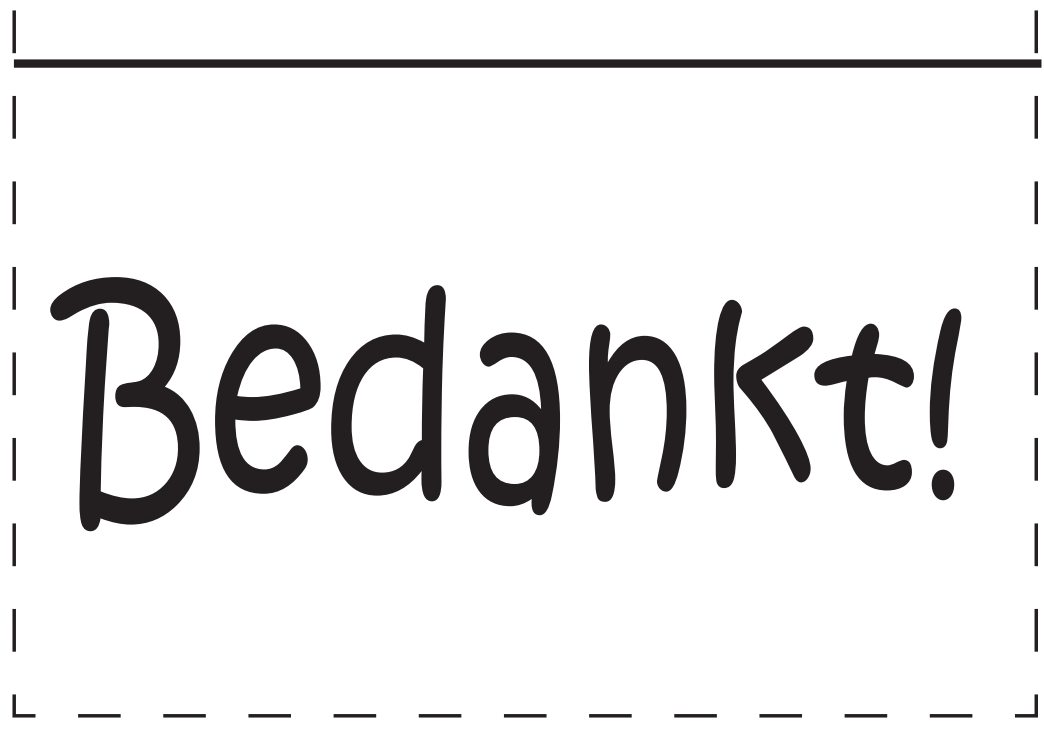

COLOR FIGURES 

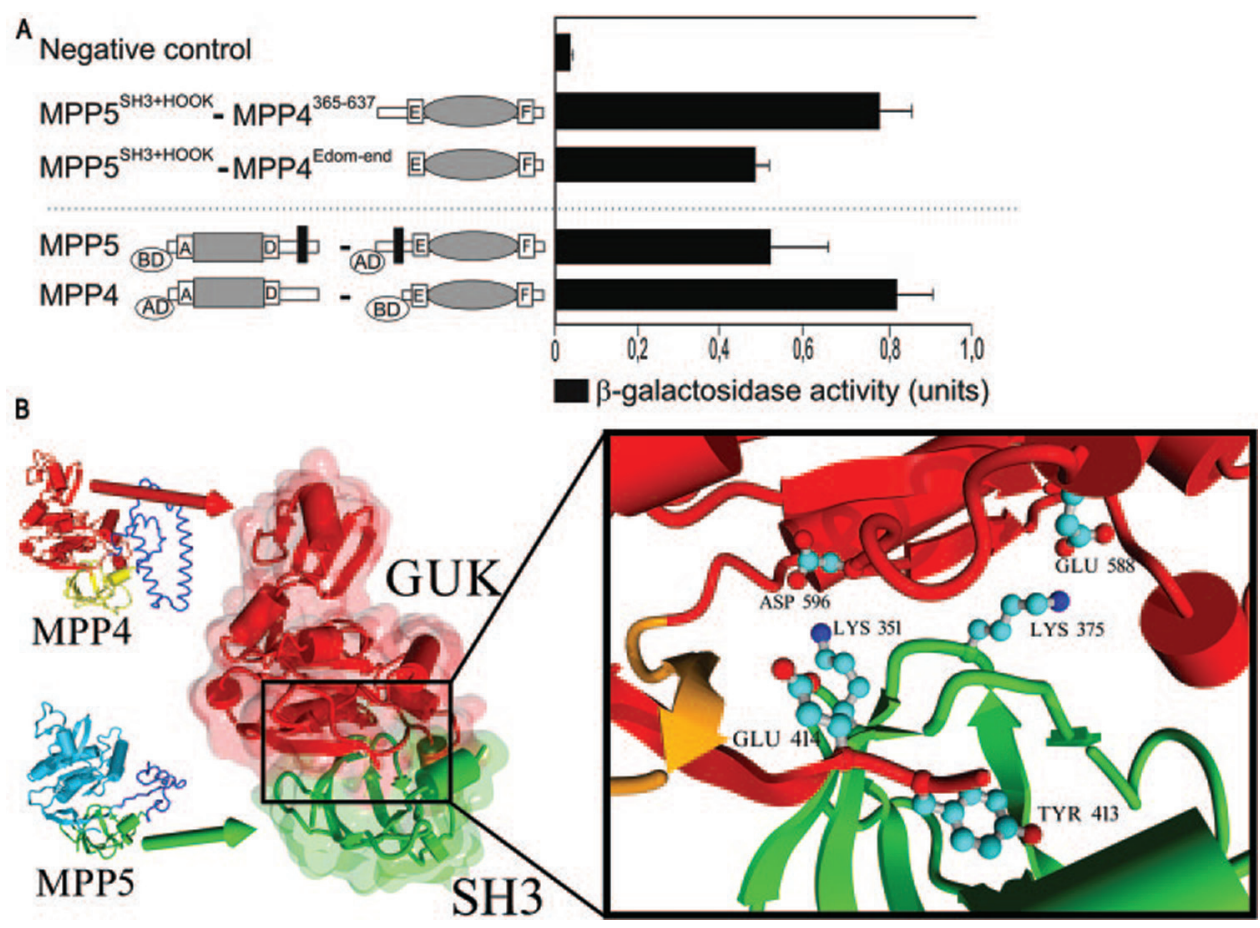

Chapter 2, Fig. 3 Analysis of the interacting domains of MPP4 and -5. (A). To quantify the intermolecular interaction between MPP4 and -5 (above dotted line), the YRG-2 yeast strain was cotransformed with the SH3-HOOK region of MPP5 together with two different deletion constructs of MPP4. The minimal region of MPP4 needed for interaction contains the E-GuK-F region. To determine the intramolecular interaction of MPP5 as well as MPP4 (below dotted line), yeast cells were co-transformed with two constructs of MPP5 (pBD-GAL4 domain fused to the SH3-HOOK region and the pADGAL4 domain fused to the HOOK-GuK domain), as well as two constructs of MPP4 (pBD-GAL4 domain fused to the GuK domain and pAD-GAL4 domain fused to the SH3 domain). Interactions were quantified by determining the activation of the LacZ reporter gene in a liquid ONPG assay ( $\beta$-galactosidase activity, black bars). As a negative control, the pBD-GAL4 domain fused to CRB1 ${ }^{\text {intra }}$ and the pAD-GAL4 domain fused to MPP4 ${ }^{\mathrm{PDZ}}$ was used. (B). Homology modeling of MPP4 and -5 . The initial models are shown on the left, covering the $\mathrm{SH} 3$ (yellow, green) and GuK domains (red, light blue). By swapping the domains, one obtains a hetero-dimer, half of which is shown in the middle: the SH3 domain of MPP5 bound to the GuK domain of MPP4. Energy calculations predicted a high binding energy for this interaction (Table 2), due to a number of salt bridges, shown in the close-up: the triad Glu $414_{\text {MPP4 }}$ - Lys $351_{\mathrm{MPP} 5}$ - Asp $596_{\mathrm{MPP} 4}$, then Lys $375_{\mathrm{MPP} 5}$ - Glu $588_{\mathrm{MPP} 4}$ and (not shown) Arg $418_{\text {MPP4 }}$ - Glu $395_{\text {MPP5 }}$. Note that an essential tyrosine (Y413) in the GUK domain of MPP4 sticks in the core of the SH3 domain of MPP5. 

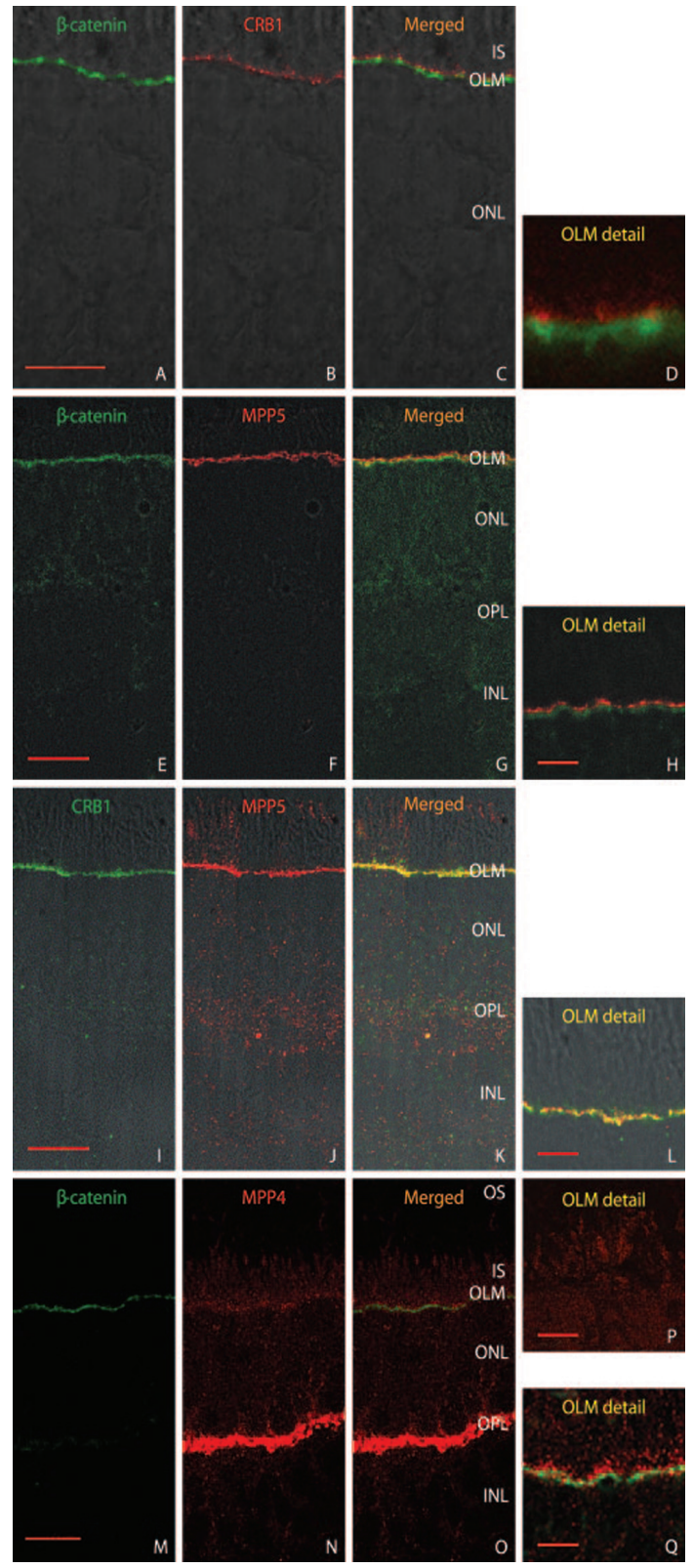

INL
Chapter 2, Fig. 5 Distribution of MPP4, MPP5, CRB1, and $B$-catenin in adult human retina.

(A-Q). Confocal images of human retinas stained with antibodies against $B$-catenin $(\mathrm{A}, \mathrm{C}-\mathrm{D}, \mathrm{E}, \mathrm{G}-\mathrm{H}, \mathrm{M}, \mathrm{O}, \mathrm{Q})$, CRB1(B-D,I,K-L), MPP5 (F$\mathrm{H}, \mathrm{J}-\mathrm{L})$, MPP4 (N-O,Q), or control secondary antibodies (P). Anti- $\beta$-catenin antibody strongly stained the adherence junction $(\mathrm{D}, \mathrm{H}, \mathrm{Q})$, whereas anti-CRB1 antibody AK2 (D,L), anti-MPP5 SN47 (H,L), and anti-MPP4 AK4 (N-O,Q) stained the SAR in the outer limiting membrane (OLM). MPP5 and CRB1 colocalize at the SAR (L). AK4 stained the OPL $(\mathrm{N}, \mathrm{O})$ and the OLM $(\mathrm{O}, \mathrm{Q})$, whereas secondary antibodies $(\mathrm{P})$ produced some background staining in the photoreceptor inner and outer segments. IS, inner segments; ONL, outer nuclear layer; OPL, outer plexiform layer; OS, outer segments; INL, inner nuclear layer. Scale bars: (A-C, H, L, P, Q) $10 \mu \mathrm{m}$; (D, E-G, I-K, M-O) $20 \mu \mathrm{m}$. 
A

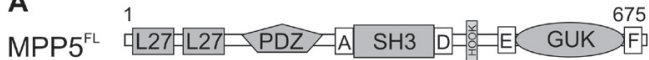

\begin{tabular}{|c|c|c|}
\hline MPP $5^{\text {SHз+нооK }}$ & $\underline{337}$ & 477 \\
\hline MPP5 $5^{\text {HooK-end }}$ & & 408 \\
\hline C & & \\
\hline pAD & $\mathrm{pBD}$ & Interaction \\
\hline MPP1 $1^{\text {prey }}$ & MPP5 $^{\text {SH3+HоOK }}$ & + \\
\hline MPP $1^{\text {Sнз+ноoK }}$ & MPP5 ${ }^{\text {Hook-end }}$ & - \\
\hline MPP5 ${ }^{\text {Hook-end }}$ & MPP $^{\text {Sнз+нооK }}$ & - \\
\hline$\overline{M P P} 1^{\mathrm{FL}}$ & $\mathrm{MPP}^{\mathrm{FL}}$ & - \\
\hline MPP1 ${ }^{\text {SНЗ+ноок }}$ & MPP1 $1^{\text {E-end }}$ & + \\
\hline MPP $1^{\text {E-end }}$ & $\mathrm{MPP}^{\mathrm{SH} 3+\mathrm{HOOK}}$ & + \\
\hline
\end{tabular}

B

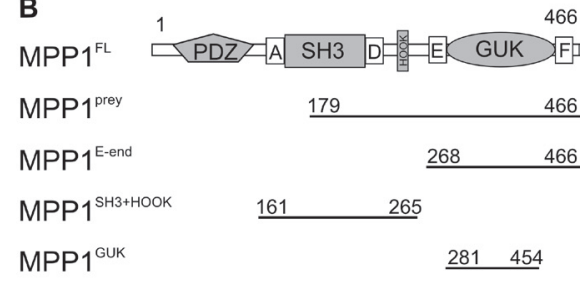

D

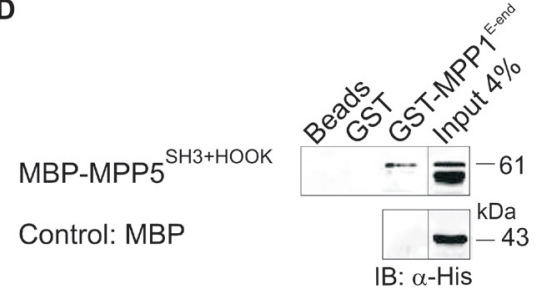

E

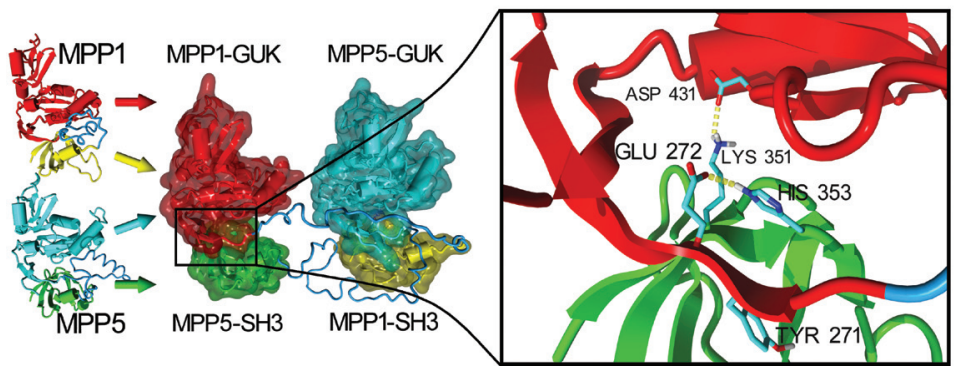

Chapter 3, Fig. 1 MPP5 binds to MPP1 by a GUK/SH3 domain swap.

(A). Schematic representation of the overall domain composition of MAGUK protein MPP5. Two L27 domains and the MAGUK module containing a PDZ, SH3 and GUK domain are present. A HOOK domain is found between the SH3 and GUK domain. Strands A and D flanking the SH3 domain and E and $\mathrm{F}$ flanking the GUK domain were identified according to homology with PSD-95. MPP5 ${ }^{\mathrm{SH} 3+\mathrm{HOOK}}$ was used as bait. The MPP5 ${ }^{\mathrm{HOOK}-\mathrm{end}}$ construct is used in a yeast two-hybrid assay to determine the specificity of the interaction between MPP1 and MPP5. (B). Identical MPP1 preys were found and MPP1 ${ }^{\text {E-end }}$, $\mathrm{MPP}^{\mathrm{SH} 3+\mathrm{HOOK}}, \mathrm{MPP}^{\mathrm{GUK}}$ and $\mathrm{MPP}^{\mathrm{FL}}$ constructs were made. $(C)$. The MPP$1^{\text {prey }}$ interacts with the MPP5 $5^{\mathrm{SH} 3+\mathrm{HOOK}}$, but MPP1 ${ }^{\mathrm{SH} 3+\mathrm{HOOK}}$ does not bind to MPP5 ${ }^{\text {HOOK-end }}$. Full-length MPP1 proteins do not bind to each other, whereas selected parts of the MAGUK modules do. (D). GST-MPP1 ${ }^{\mathrm{E}-\text { end }}$ pulled down MPP5 ${ }^{\mathrm{SH} 3+\mathrm{HOOK}}$. Beads alone or GST did not interact with MPP5, while MPP1 did not bind the MBP tag in the control experiment, showing that this interaction is specific. $(E)$. Homology modeling of the MAGUK modules of MPP1 and MPP5. The interacting GUK and SH3 domains of MPP1 (red and yellow, respectively) and MPP5 (cyan and green) separate and form a heterodimer consisting of two mixed GUK/SH3 complexes. The domain linker regions in 

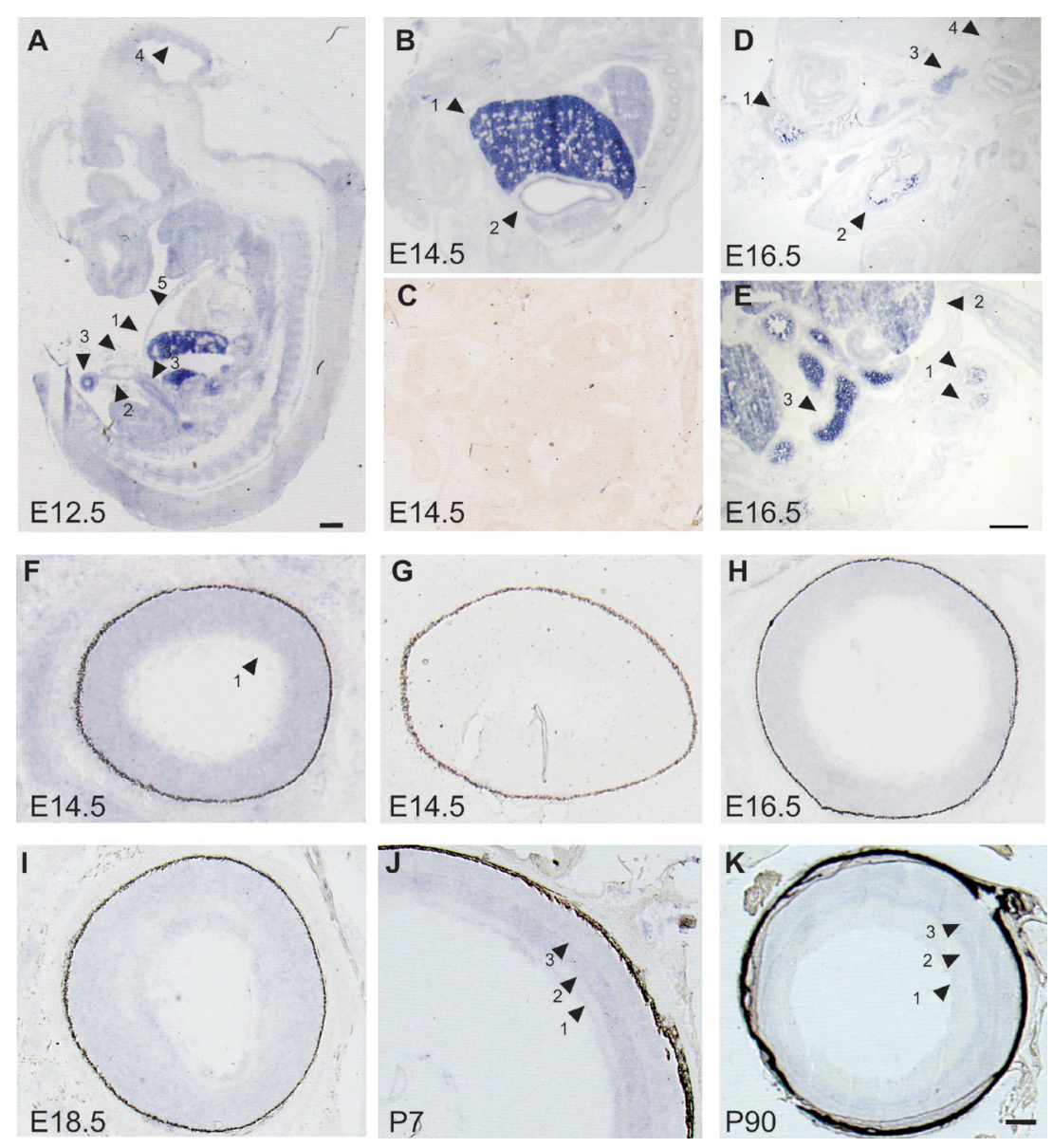

Chapter 3, Fig. 2 MPP1 expression analysis by RNA in situ hybridisation on embryonic and adult mouse tissues.

In sagittal whole embryo cryosections low levels of expression were seen throughout the embryo. (A) MPP1 expression at E12.5 in 1. liver; 2. umbilical vein; 3. primitive gut; 4. neuroepithelium; and 5. upper jaw region. Scalebar is $300 \mu \mathrm{m}$. (B) At E14.5, expression was maintained in 1. the liver; and 2. stomach. (C) Hybridisation with a MPP1 sense probe did not show tissue labeling. (D) At E16.5, the 1. zygomatic bone; 2. lower jawbone; 3. trigeminal (V) ganglion; and 4. cochlea showed expression of MPP1. (E) A strong signal was also observed in 1. femur; 2. liver; and 3. small intestine at this stage. Scalebar is $20 \mu \mathrm{m}$. (F) In the eye, a slightly higher signal intensity compared to surrounding tissues was observed in the inner neuroblastic layer. $(G)$ No staining was observed in the eye after sense probe hybridisation. (H) At E16.5 and (I) E18.5 expression was maintained in the neuroblastic layer and appeared in the developing photoreceptor layer. (J) At P7 and (K) P90, expression in 1. ganglion cells; 2.inner nuclear layer (INL) and 3. photoreceptor cell layer was seen. Scalebar is $20 \mu \mathrm{m}$. 


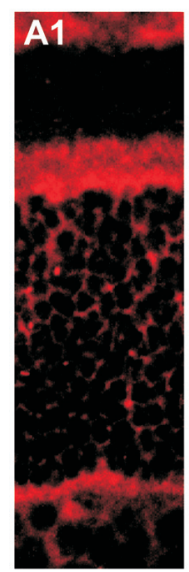

MPP1

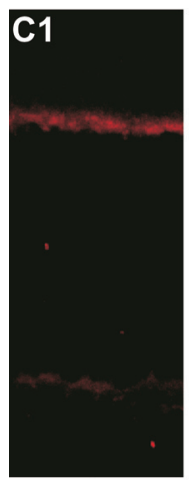

MPP5

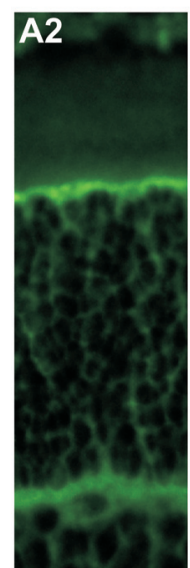

$\beta$-catenin

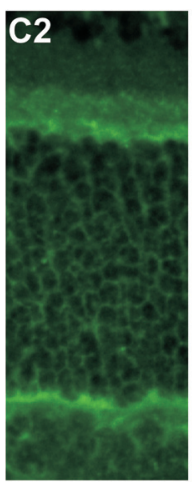

MPP1

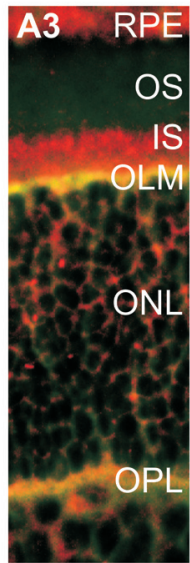

Merged

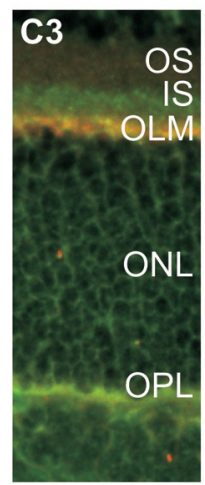

Merged

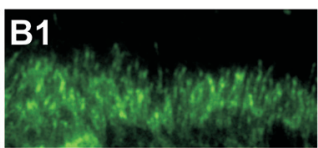

MPP1

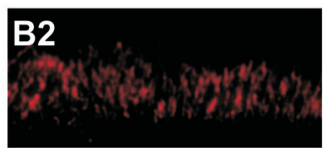

Acetylated tubulin

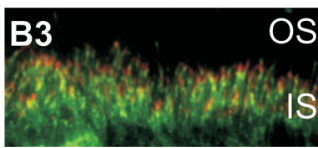

Merged
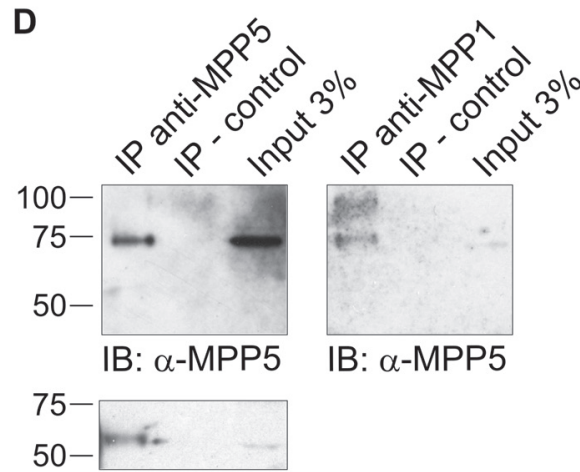

IB: $\alpha-M P P 1$

Chapter 3, Fig. 3 MPP1 and MPP5 interact at the outer limiting membrane of the neural retina.

(A). Staining of retinal cryosections using MPP1 rabbit serum (red) detected the protein at the OPL, ONL, OLM, inner segments, RPE and at the connecting cilium region. (A2). Costaining with an anti- $\beta$-catenin antibody (green signal), a marker for the OLM and OPL, confirmed the presence of MPP1 at these locations, as shown by the yellow signal in the overlay (A3). (B). Costaining of MPP1 with an antibody against the connecting cilium marker acetylated tubulin. (C1). Anti-MPP5 only stained the OLM, and costaining with anti-MPP1 $(C 2)$ indicates their co-localization in the overlay $(C 3)$. (D). Immunoprecipitation with MPP5 antibody precipitates MPP5 $(75 \mathrm{kD})$ as well as MPP1 $(55 \mathrm{kD})$ from bovine retina (left panels, IP control is $\operatorname{IgG}$ ). In the reverse experiment, MPP1 antibody coimmunoprecipitates MPP5 from mouse retina (right panel). 
D

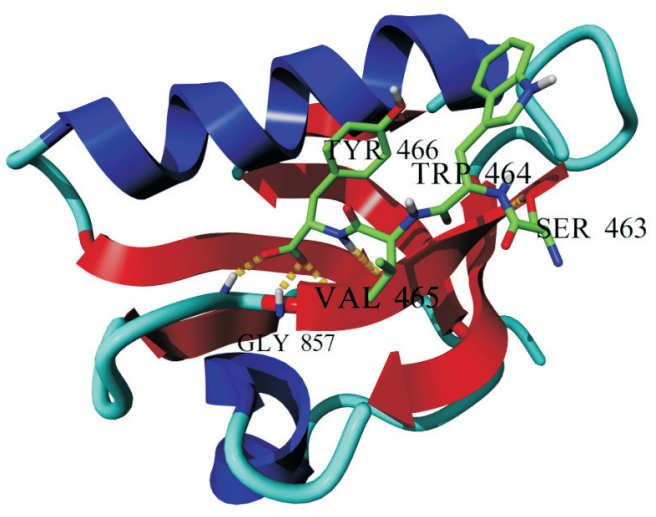

Chapter 3, Fig. 4 Whirlin can bind to MPP1 by two different mechanisms. $(D)$. Homology modeling of the MPP1 C-terminus bound to the C-terminal whirlin PDZ3 domain (PDB 1UFX) indicates that the core motif of the interaction is the peptide's C-terminal carboxyl group tightly bound by three backbone NH groups, a common feature in PDZ-PBM interactions. However, this interaction is not possible in 1UFX (the whirlin PDZ structure) since one peptide plane is flipped and a $\mathrm{C}=\mathrm{O}$ faces the carboxyl group. Therefore, it is predicted that Gly 857 in whirlin must undergo a conformational change to allow binding, which is represented in the figure. 


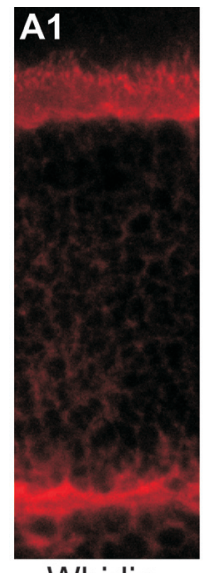

Whirlin

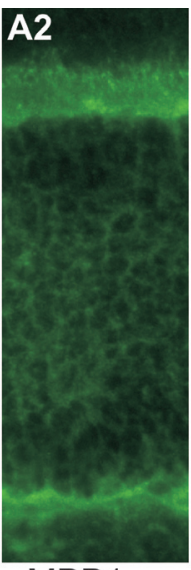

MPP1

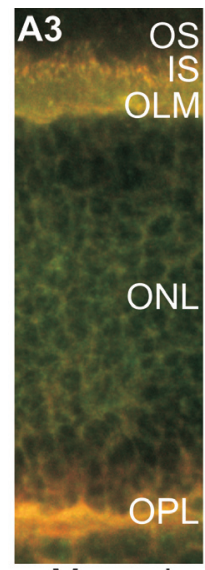

Merged
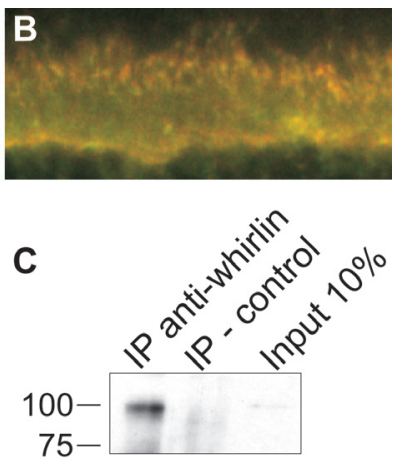

IB: $\alpha$-whirlin

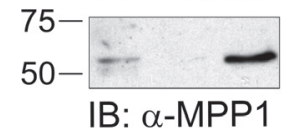

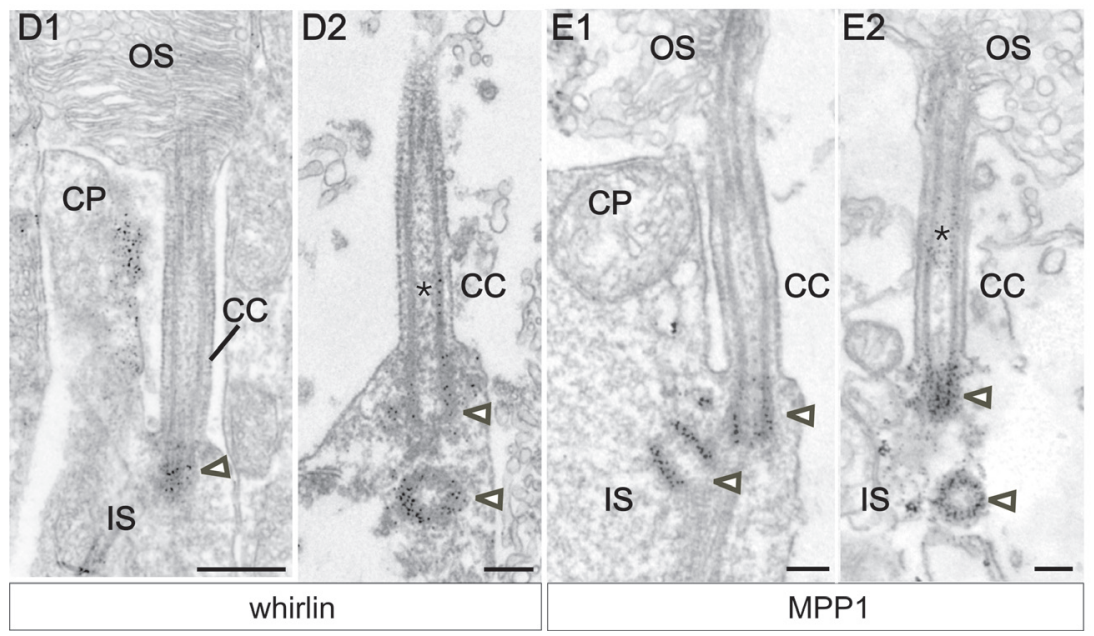

Chapter 3, Fig. 5 MPP1 interacts with whirlin in photoreceptors. $(A-B)$. Retinal cryosections were stained using polyclonal antibodies against whirlin $(A 1)$ and MPP1 (A2). The signals show a near-complete overlap in the overlay (A3). (B). The most prominent staining was found at the OPL, OLM and around the inner segments (IS). (C). Immunoprecipitation of whirlin using a polyclonal anti-whirlin antibody precipitated whirlin from mouse retinal lysates (top). MPP1 co-precipitated with anti-whirlin (bottom). As a control, protein A/G agarose beads without primary antibody were used. (D). Pre-embedding immunolabeling of the ciliary region of mouse photoreceptors by antibodies against whirlin shows a clear staining of the calycal processes (CP), the basal bodies (arrow heads) (D1 and D2) and of the connecting cilium (indicated by asterisks) (D2). Sections stained with anti-MPP1 show an identical signal in the basal bodies and the connecting cilium (E1 and E2), but not in the calycal process. Bars in D1 $0.5 \mu \mathrm{m}$; D2 $0.25 \mu \mathrm{m}$; E1 and E2 $0.2 \mu \mathrm{m}$. 

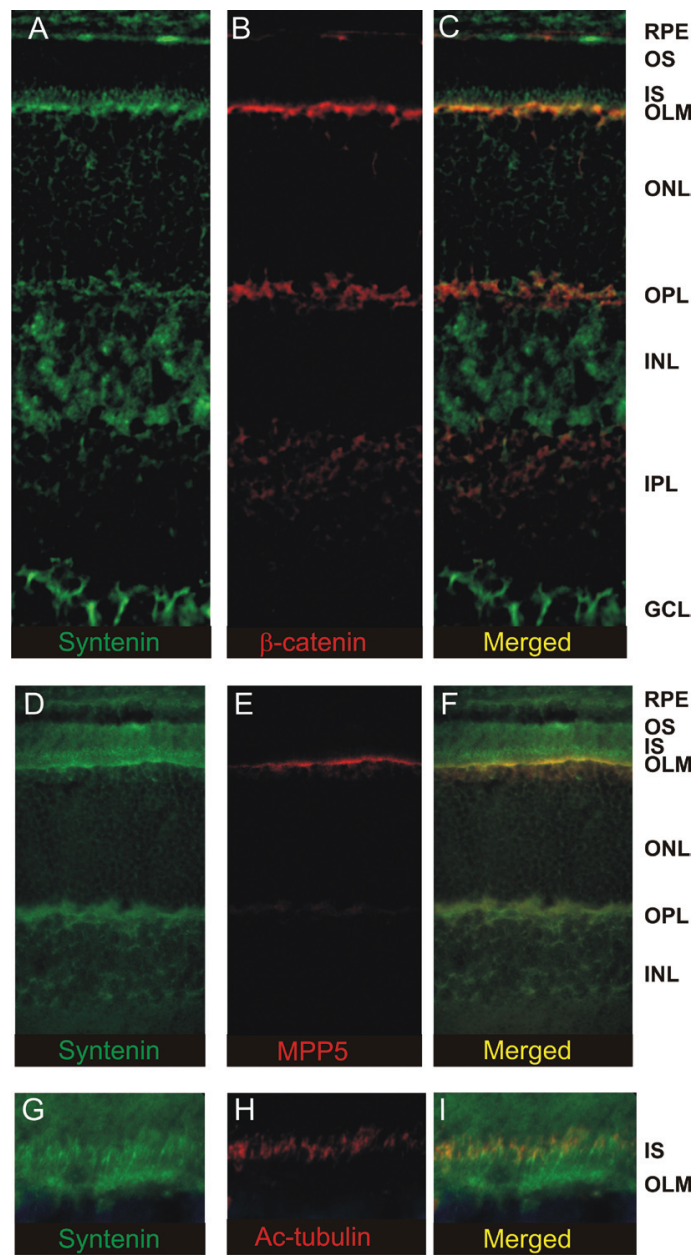

Chapter 4, Fig. 3 Localization of syntenin-1 in rat retina.

(A). Syntenin-1 (green) staining using the C96 antibody was found in the retinal pigment epithelium (RPE), inner segments (IS), outer plexiform layer (OPL), outer nuclear layer (ONL), inner nuclear layer (INL) and ganglion cell layer (GCL) and outer limiting membrane (OLM). (B and C). Co-localization with $\beta$-catenin (red), a marker for adherens junctions was found at the OLM. (D).A similar staining pattern for syntenin-1 was observed using the SySy anti-syntenin-1 antibody. (E). MPP5 staining was detected exclusively at the OLM, as previously shown [10]. (F). Co-localization of syntenin-1 (green) with MPP5 (red) was found at the OLM. (G). In the inner segments (IS) a punctate staining pattern of syntenin-1 was observed. $(\mathrm{H})$. Anti-acetylated tubulin antibody, a marker for the ciliary axoneme, stained microtubule fibers of the connecting cilium. (I). Acetylated tubulin and syntenin-1 did not reveal complete overlap, but co-localization was observed at the region of the connecting cilium. 


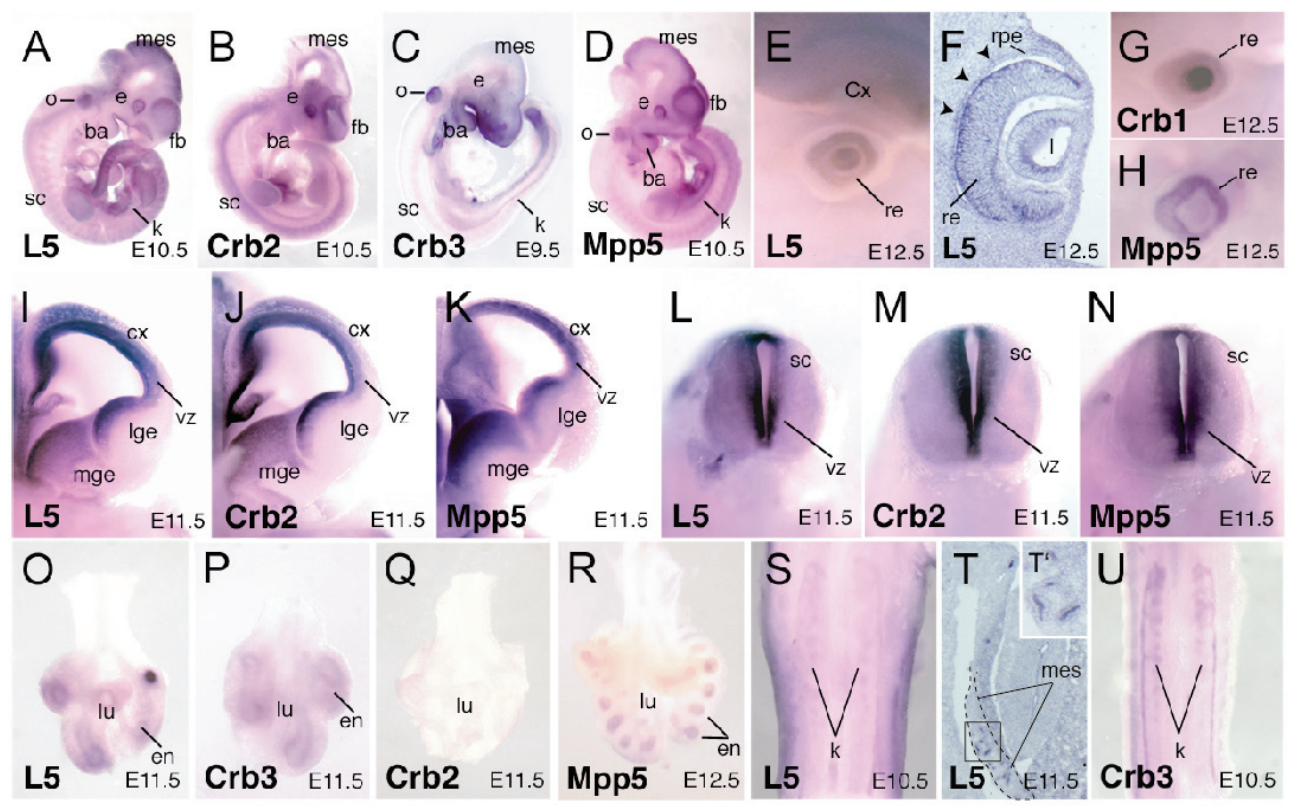

Chapter 5, Fig. 1 Crb1, Crb2, Crb3, Mpp5 and Epb4.115 (L5) RNA localization in mouse embryos at different developmental stages. (A-D). Expression pattern of Epb4.115 (A), Crb2 (B), Crb3 (C) and Mpp5 (D) in E9.5-E10.5 whole embryos. Epb41L5 and Mpp5 are co-expressed in different tissues like the forebrain (fb), mesencephalon (mes), spinal cord (sc), eye (e), branchial arches (ba), otic vesicle (o) and kidney (k). In all these tissues, either Crb2 (B) or Crb3 (C) or both are expressed. (E-H). Expression in the developing eye of Epb4.115 (E-F), Crb1 $(\mathrm{G}), M p p 5(\mathrm{H})$ is co-localizing in the retina. Epb4.115 transcripts are not detected in the retinal pigmented epithelium (rpe) (arrowheads in F). Expression of Epb4.115, Crb2 and Mpp5 in the developing forebrain (I-K) and spinal cord $(\mathrm{L}-\mathrm{N})$ in E11.5 dissected mouse neural tube. (O-R) Note Epb4.115 (O), Crb3 (P), Crb2 (Q), Mpp5 (R) expression in developing lungs (lu) isolated from E10.5 and E11.5 mouse embryos. All genes, except $C r b 2$, are expressed in the endodermal layer (en) of the forming bronchioles (Epb4.115, Crb3) or saccules $(M p p 5)$. Both Epb4.115 (S-T), and Crb3 (U) expression is detected in the mesonephric tubules of the developing kidney $(\mathrm{k})$ in E10.5-E11.5 mouse embryos. Dashed lines in T delineate the mesonephric tissue. ( $T$ ') High magnification of the boxed-area in ( $\mathrm{T}$ ) showing Epb4.115 expression in the endothelial cells of the mesonepric tubules. $\mathrm{Cx}$, cerebral cortex; lge, lateral ganglionic eminence; mge, medial ganglionic eminence; vz, ventricular zone. 


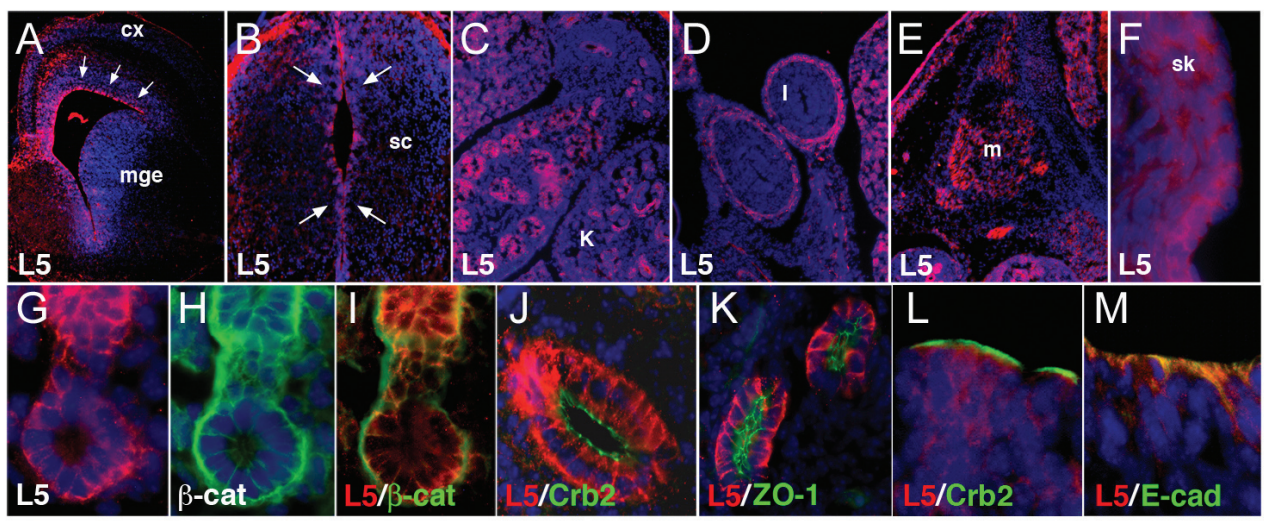

Chapter 5, Fig. 2 Epb4.115 protein distribution in embryonic tissues and cellular localization. Cross sections of E15.5 cerebral cortex (A) and spinal cord (B) where Epb4.115 was found to be highly expressed. Strong staining is detected in the luminal side of the entire neural tube fading moving from the ventricle to the peripheral parenchyma (arrows in A, B). Epb4.115 was found to localize in E15.5 embryonic kidney (C), intestinal guts (D), majority of the developing muscles (E) and deep layers of the overlaying immature skin (F). (G-K) Close view of Epb4.115 localization in kidney epithelial tubules. (G-I) Epb4.115 and $\beta$-catenin double staining on a renal tubule cross-section. Both stainings appeared largely overlapping on the basolateral cell membrane domains (I). $(\mathrm{J}, \mathrm{K})$ Double staining of Epb4.115 with proteins of the cell membrane apical domain such as Crb2 on sections of renal tubules $(\mathrm{J})$ and ZO-1 $(\mathrm{K})$ revealed a close association of both membrane domains without any notable overlap. (L, M) Close view of the cerebral cortex proliferative layer overlying the ventricle. Cortical proliferative cells are forming a pseudo-epithelial structure tightened by intercellular junctions. Epb4.115 staining is strongly detected in a basolateral cellular domain overlapping with E-cadherin (M), but excluded from the most cortical apical side lining the ventricle and stained positive for Crb2 (L). 

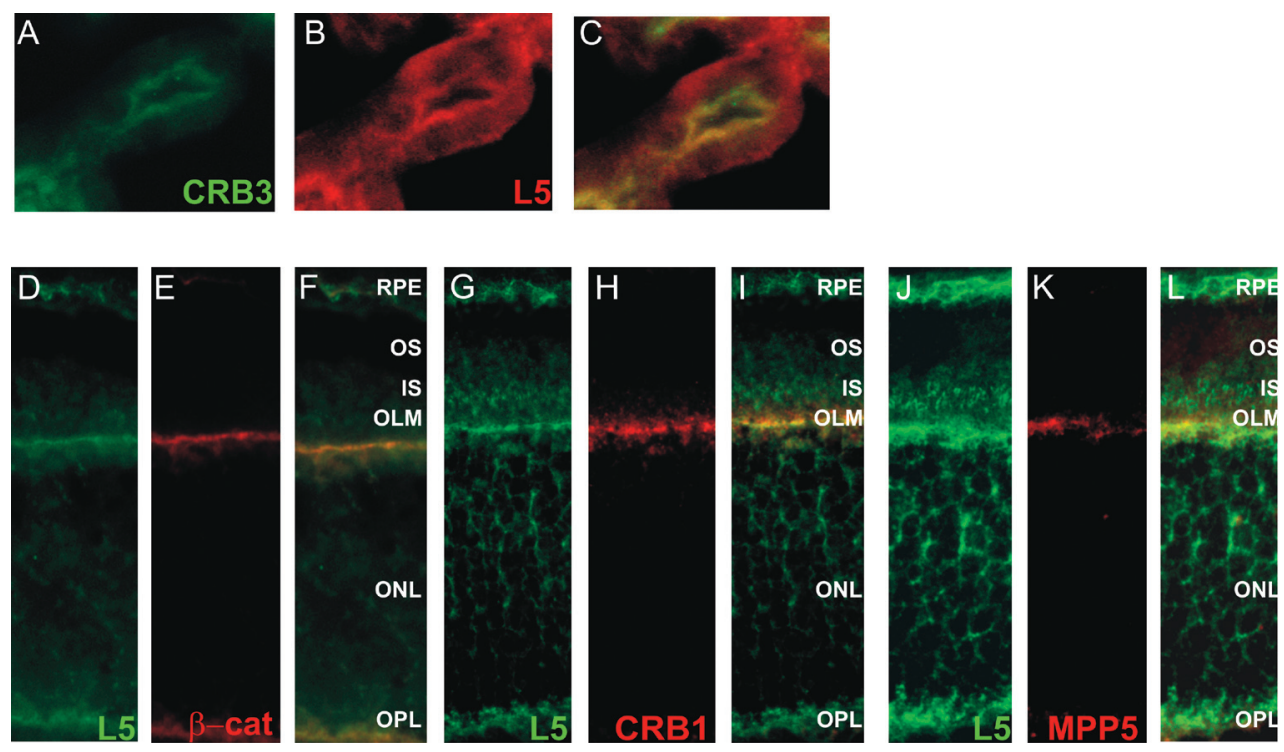

Chapter 5, Fig. 3 Co-localization of Epb4.1L5 with CRBs and Mpp5 in adult tissues. (A). Crb3 (green) is found only at the apical side of the kidney tubule, while (B) Epb4.115 (L5, red) is found at the basal and the apical side in adult kidney. (C). Overlap in staining is found at the apical side. (D, G, J) In the retina, Epb4.115 (L5, green) is found in the retinal pigment epithelium (RPE), outer plexiform layer (OPL), outer nuclear layer (ONL), inner segments (IS) and outer limiting membrane (OLM). (D-F) Co-localization with $\beta$-catenin (red), a marker for adherens junctions is found at the OLM. (G-I) Also co-localization of Epb4.1L5 (green) with CRB1 (red) and (J-L) with MPP5 (red) is found at the OLM. 


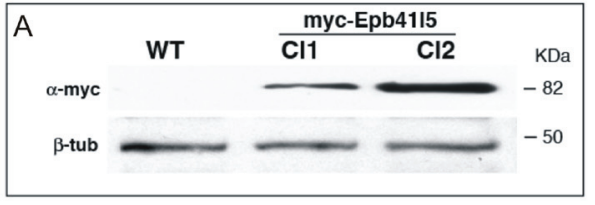

B

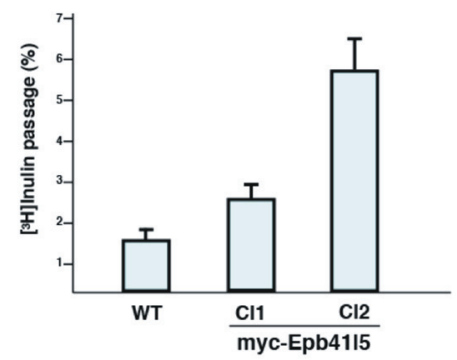

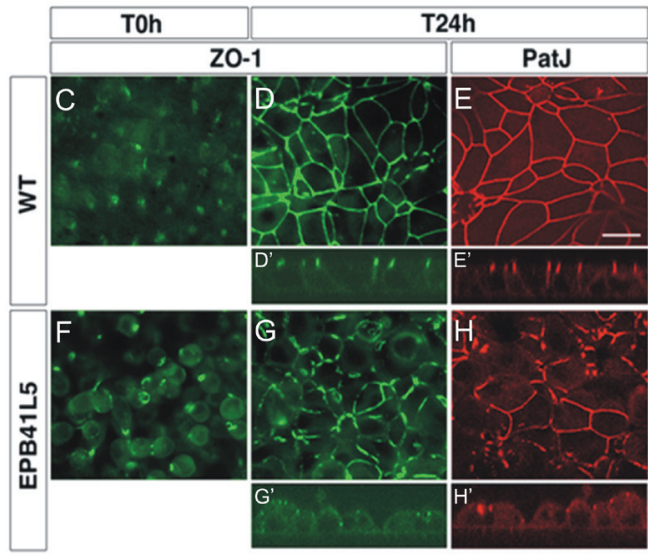

Chapter 5, Fig. 7 Overexpression of Epb4.11L5 affects tight junction formation in calcium-switch dependent MDCK cell re-polarization. (A). Western-blot with an anti-myc antibody showing stable MDCK cell lines expressing low (clone 1) and high (clone 2) levels of the myc-Epb4.115 transgene. (B). Analysis of cell monolayer tightness by $\left[{ }^{3} \mathrm{H}\right]$ inulin leakage assay, 12 hours after calcium switch. Clones 1 and 2 expressing myc-Epb4.115 show about 1.6 4 time fold leakage of $\left[{ }^{3} \mathrm{H}\right]$ inulin compared to control cells in a transwell chamber, respectively. (C-H) Analysis of tight junction assembly in control (C-E) and clone 2 overexpressing myc-Epb4.115 (F-H) cells in a "calcium-switch" assay. (C, F) Complete loss of polarity and junction structures in cells treated with a medium lacking $\mathrm{Ca}^{2+}(\mathrm{T}=0)$ is seen. Tight junctions are completely restored in control cells from a change in high $\mathrm{Ca}^{2+}$ culture medium after 12 hours, as visualized with ZO-1 and PATJ protein localization (D, D', E, E'). Conversely, clone 2 expressing myc-Epb4.115 cells display a severe cellular disorganization and impairment in tight junction assembly as shown by loss of ZO-1 and PATJ proteins in cell borders (arrows in G, G', H, H'). D', E', G', H', Z-sections showing the apical-basolateral localization of the proteins. Bar, $10 \mu \mathrm{m}$. 

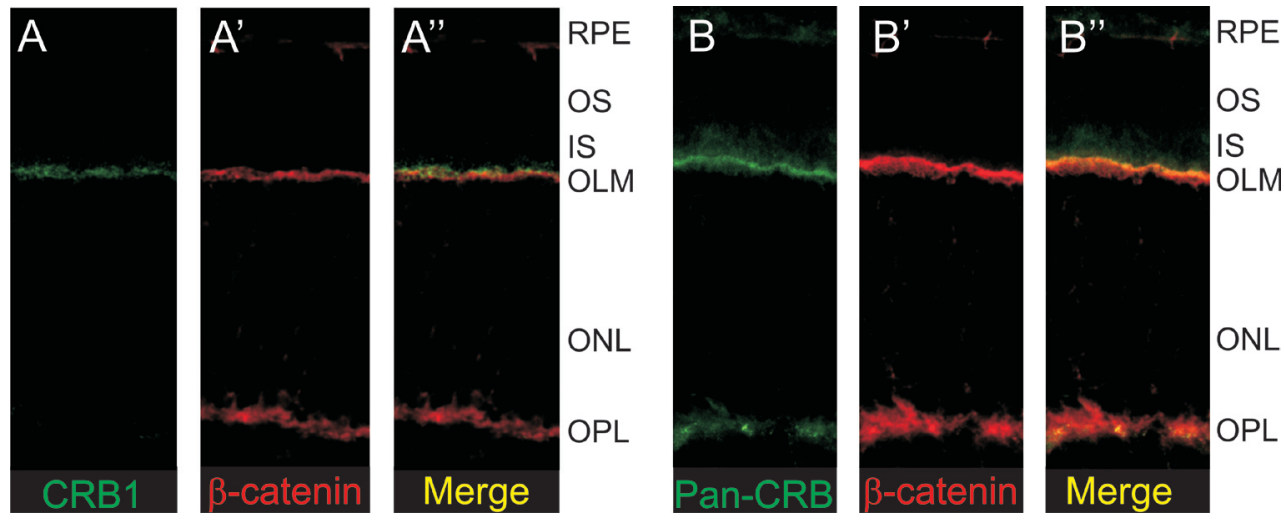

Chapter 6, Fig. 6 Immunohistochemistry of CRB proteins in the retina. A: CRB1 localizes to the OLM and is not found in the synapses. B: An antibody that recognizes all three CRB orthologues (pan-CRB) stains the OLM and the OPL. Immunohistochemistry was performed as described in addendum 2 . 
